\title{
DETECTION AND EFFECTS OF LATENT CONTAMINATION OF POTATO TUBERS BY SOFT ROT BACTERIA, AND \\ INVESTIGATIONS ON THE EFFECT OF HYDROGEN PEROXIDE ON \\ LIPOPOLYSACCHARIDES OF ERWINIA CAROTOVORA IN RELATION TO ACQUIRED RESISTANCE AGAINST BIOCIDES
}

\author{
Doctoral Dissertation \\ Submitted for the degree of Doctor of Agricultural Sciences \\ of the Faculty of Agricultural Sciences \\ Georg-August-University, Göttingen, Germany \\ By \\ Mamdoh Ewis Esmael Ahmed \\ Born in Beni Mazar, El-Minia, Egypt \\ Institute of Plant Pathology and Plant Protection \\ Faculty of Agriculture \\ Georg-August-University, Göttingen, Germany
}

Göttingen, November 2001 
D7

1. Referent: $\quad$ Prof. Dr. Hoppe

2. Korreferent: Prof. Dr. Eberhardt

Tag der mündlichen Prüfung: 22. 11. 01 


\section{TABLE OF CONTENTS}

\section{CHAPTER I}

Detection of Erwinia carotovora in potato stocks using a semiselective medium

1 INTRODUCTION

2 MATERIAL AND METHODS

2.1 Media for bacteria 3

2.1.1 Yeast-dextrose-chalk agar (YDC) 3

2.1.2 King's medium B 3

2.1.3 Crystal violet pectate medium (CVPB) 3

2.1.4 Double layer CVP medium (DLCVP) 4

2.1.5 Crystal violet pectate double layer medium (Bdliya) 4

2.1.6 $\alpha$-Methyl-d-glucoside medium 5

2.1.7 Phosphatase detection medium 5

2.1.8 Medium for indole production 5

2.1.9 Kovacs' indole reagent 5

$\begin{array}{lll}2.1 .10 & \text { Nutrient agar (NA) }\end{array}$

2.1.11 Gas production from glucose 6

2.1.12 Logan's medium 6

$\begin{array}{lll}2.1 .13 & \text { Lecithinase activity }\end{array}$

2.1.14 Salt tolerance 7

2.1.15 Mineral salt medium 7

$\begin{array}{lll}\text { 2.1.16 Antibiotics used in this study } & 7\end{array}$

$\begin{array}{lll}2.2 & \text { Characterization of Erwinia spp } & 8\end{array}$

$\begin{array}{lll}\text { 2.2.1 } & \text { Bacterial strains used in these studies } & 8\end{array}$

2.2.2 Isolation of Erwinia spp. from different potato specimens 8

$\begin{array}{lll}\text { 2.2.3 Conventional physiological tests } & 9\end{array}$ 
2.2.3.1 Utilization of $\alpha$-methyl-d-glucoside

2.2.3.2 Phosphatase production

2.2.3.3 Differentiation between Erwinia spp. by growth at different temperatures 9

$\begin{array}{lll}\text { 2.2.3.4 Indole production } & 10\end{array}$

$\begin{array}{lll}\text { 2.2.3.5 Gas production from glucose } & 10\end{array}$

$\begin{array}{lll}\text { 2.2.3.6 Logan’s medium } & 10\end{array}$

$\begin{array}{lll}\text { 2.2.3.7 Lecithinase activity (Egg yolk test) } & 10\end{array}$

$\begin{array}{lll}2.2 .3 .8 & \text { Salt tolerance } & 10\end{array}$

$\begin{array}{lll}\text { 2.2.4 Biotest on potato tuber slices } & 11\end{array}$

2.2.5 Maintenance and storage of bacterial cultures 11

2.3 Development of a new semi-selective medium for

E. carotovora

2.3.1 Effect of tryptone in the basal and over layer on the growth of Erwinia spp. on the semi-selective medium

2.3.2 Effect of tri-sodium citrate dihydrate in the basal and over layer on the growth of Erwinia spp. on the semi-selective medium

2.3.3 Comparison of different pectin sources for isolation of Erwinia spp. $\begin{array}{ll}\text { on the semi-selective medium } & 12\end{array}$

2.3.4 Evaluation of bacterial growth on the semi-selective medium 13

2.3.5 Recovery of Erwinia spp. from artificially inoculated potato extracts on semi-selective media and on King's medium B

2.3.6 Detection limit of Erwinia spp. on the semi-selective media and on King's medium B

2.3.7 Isolation of Erwinia spp. on the new semi-selective medium

2.3.7.1 Potato peel extracts 
2.4 Evaluation of latent contamination of 232 potato tuber samples

from the years 1998, 1999 and 2000

2.5 Field experiments

3 RESULTS

3.1 Preservation of the Erwinia spp. used in this study

3.2 Development of a semi-selective medium for isolation of Erwinia spp.

3.2.1 Effect of tryptone and tri-sodium citrate dihydrate in the basal and over layer on the growth of Erwinia spp. on the semi-selective medium

3.2.2 Effect of different pectin sources on the growth of Erwinia spp.

3.2.3 Composition of the new semi-selective medium (CVPM)

for isolation of soft rot erwinias

3.3 Recovery rate and detection limit of Erwinia spp. from artificially inoculated potato extracts

3.4 Application of the new semi-selective medium for detection of Erwinia spp. from potato tubers using two different methods

3.5 Isolation and identification of erwinias from potato tubers

3.5.1 Identification of soft rot erwinias $(E c a, E c c$ and $E c h)$ by physiological tests 23

3.5.2 Identification of Erwinia spp. from different potato specimens

3.6 Detection of pectolytic Erwinia spp. on single potato tubers and in groups of potato tubers

3.7 Detection of latent contamination of with Erwinia carotovora ssp. 
in different potato tuber samples from Stoever Produktion GmbH

\& Co. KG

3.7.1 Comparison between laboratory tests and field observations

3.7.2 Evaluation of latent contamination of 232 potato tuber samples from the years 1998, 1999 and 2000

3.7.3 Evaluation of potato lots with different degrees of latent contamination by field experiments in the following year

4 DISCUSSION

4.1 Identification and differentiation of Erwinia spp.

4.2 Preservation of Erwinia spp. strains used in this study

4.3 Development of a semi-selective medium for isolation of soft rot erwinias

4.4 Comparison of different pectin sources for detection of Erwinia spp. on the semi-selective medium

4.5 Recovery rate and detection limit of erwinias in artificially contaminated potato homogenates

4.6 Occurrence of different erwinias

4.7 Evaluation of methods to identify and quantify latent contamination of potatoes by erwinias

4.8 Sample size

4.9 Comparison of different years

4.10 Comparison of field observations and laboratory tests

4.11 Field experiments with potato lots assessed in different degrees of latent contamination by Ecc 


\section{CHAPTER II}

Effect of Degaclean on the reduction of soft rot of potato tubers

1 INTRODUCTION

2 MATERIAL AND METHODS

2.1 Selection of antibiotic resistant isolates from Erwinia spp. 52

2.2 Selection of Degaclean tolerant isolates of Erwinia spp.

2.3 Inoculation of potato tubers with Erwinia spp. by wounding

2.4 Effect of Degaclean on potato tubers infected by

Erwinia spp.

2.4.1 Origin of Degaclean 53

$\begin{array}{lll}\text { 2.4.2 Laboratory experiments } & 54\end{array}$

$\begin{array}{lll}\text { 2.4.3 Field experiments } & 54\end{array}$

2.4.3.1 Field experiments in Göttingen $\quad 54$

2.4.3.1.1 Evaluation at harvest $\quad 59$

$\begin{array}{ll}\text { 2.4.3.2 Field experiments by Stöver Co. } & 59\end{array}$

2.5 Characterization of lipopolysaccharides from Erwinia carotovora strains

2.5.1 Culture of bacteria and washing of cells 60

2.5.2 Extraction of LPS by the phenol-water method 60

$\begin{array}{lll}\text { 2.5.3 } & \text { Polyacrylamide gel electrophoresis (PAGE) }\end{array}$

2.5.3.1 Preparation of separation gel $\quad 61$

2.5.3.2 Preparation of stacking gel 61

2.5.3.3 Buffers, chemicals and reagents for electrophoretic studies 62

2.5.3.4 Preparation of LPS samples 63 
3 RESULTS

3.1 Effect of Degaclean on reduction of potato soft rot in laboratory experiments

3.1.1 Development of a standardized method for inducing soft rot by artificial contamination of potato tubers

3.1.2 Disease severity by Erwinia spp. after treatment of potato tubers with different concentrations of Degaclean

3.2 Effect of Degaclean on reduction of blackleg and potato soft rot in field experiments

3.2.1 Field experiments 1999 66

3.2.1.1 Field experiments in Göttingen 66

3.2.1.2 Field experiments by Stöver Co.

3.2.2 Field experiments 2000 68

\subsection{Generation of Degaclean tolerant strains of Erwinia} carotovora ssp. carotovora and ssp. atroseptica

3.3.1 Sensitivity against different concentrations of Degaclean

3.3.3 Effect of Degaclean-tolerance on LPS

4 DISCUSSION

4.1 Comparison of methods for artificial inoculation

4.2 Effect of Degaclean on reduction of soft rot in laboratory experiments 
$\begin{array}{lll}\text { 4.3.2 } & \text { Effect of Degaclean on soft rotting of potato tubers } & 75\end{array}$

4.3.3 Effect of Degaclean-treatment on potato yields $\quad 76$

4.3.4 Effect of Degaclean on reduction of latent contamination of potatoes $\quad 76$

4.3.5 Differentiation of $E c a$ and $E c c$

4.4 Effect of Degaclean-tolerance on LPS structure $\quad 77$

$\begin{array}{lll}5 & \text { SUMMARY } & 78\end{array}$

\section{CHAPTER III}

Chemical structure of the LPS-O-chain of Erwinia carotovora and its possible role in virulence

$1 \quad$ INTRODUCTION 79

2 MATERIAL AND METHODS 82

2.1 LPS characterization $\quad 82$

$\begin{array}{lll}\text { 2.1.1 } & \text { Extraction and purification } & 82\end{array}$

2.1.2 SDS polyacrylamide gel electrophoresis (PAGE) 82

$\begin{array}{lll}2.2 & \text { Structure analysis of the LPS-O-chain } & 82\end{array}$

2.3 Determination of the virulence of two Eca-strains 83

$\begin{array}{lll}\text { 2.3.1 } & \text { Laboratory experiments } & 83\end{array}$

$\begin{array}{lll}\text { 2.3.2 } & \text { Greenhouse experiments } & 83\end{array}$

2.4 Determination of pectolytic activity of two Eca-strains $\quad 84$

2.4.1 Multiplication of two Eca-strains of in pectin and King's B

liquid medium

2.4.2 Determination of bacterial growth of two $E c a$ strains using the semi-selective medium (CVPM) 
$3 \quad$ RESULTS

3.1 Analysis of lipopolysaccharides (LPS)

3.1.1 Characterization by SDS-PAGE

3.1.2 Structure of the LPS-O-chain of Ecc (GSPB 436) 88

3.1.3 Structure of the LPS-O-chain of Eca (GSPB 2967) 88

3.2 Virulence test of two Erwinia carotovora ssp. atroseptica strains on potato tubers slices and Chinese cabbage $\quad 88$

$\begin{array}{lll}\text { 3.2.1 } & \text { Tests on potato slices in Petri dishes } & 88\end{array}$

3.2.2 Tests on potato plants in pots in the greenhouse 89

3.2.3 Tests on leaves of Chinese cabbage, tomato, potato and tobacco and on $\begin{array}{ll}\text { thick leaves of Chinese cabbage heads } & 90\end{array}$

$\begin{array}{lll}3.3 & \text { Pectolytic activity } & 93\end{array}$

3.4 Multiplication of Eca-strains in/on different media 95

$\begin{array}{lll}\text { 3.4.1 In pectin medium } & 95\end{array}$

$\begin{array}{lll}\text { 3.4.2 In KB medium } & 96\end{array}$

3.4.3 Comparison of the growth of two Eca strains on the CVPM medium 97

4 DISCUSSION 98

4.1 Structure of the LPS-O-chain of Erwinia carotovora ssp. carotovora 99

4.2 Preliminary structure of the LPS-O-chain of Erwinia carotovora ssp. atroseptica

4.3 Virulence of the Japanese "rough" Eca-strain in comparison to other "smooth" Erwinia carotovora strains 


\section{CHAPTER IV}

Comparison of two primers for the detection of Erwinia carotovora ssp. atroseptica and Erwinia carotovora ssp. carotovora in potato tubers using the polymerase chain reaction (PCR)

1 INTRODUCTION

2 MATERIAL AND METHODS

2.1 Solutions and buffers for agarose gel electrophoresis

2.3 Analysis of the PCR product using agarose

gel electrophoresis

2.4 Determination of the detection limit of the PCR method

2.5 Determination of $E c a$ and $E c c$ with specific primers

2.6 Effect of potato peel extract on the detection limit of Eca by PCR

2.6.1 DNA isolation by using the method of LI and DE BOER

2.6.2 DNA isolation by using the method of MÖLLER

2.6.3 DNA isolation by using the $\mathrm{NaOH}$ method

2.6.4 DNA isolation by using the method of the German Plant Protection Service, Hannover

3.1 Detection and identification of Erwinia spp. using the 
3.2 The detection limit of the PCR method using primers Y1 and $\mathrm{Y} 2$

3.3 The detection limit of the PCR method using primers Eca1f and Eca2r

3.4 Detection of Eca in artificially contaminated potato peel extract by PCR with primers Y1 and Y2

3.5 Detection of $E c a$ in artificially contaminated potato peel extract by PCR with primers Eca1f and Eca2r

4 DISCUSSION

4.1 Specificity of the two $E c$ primer pairs

4.2 Determination of the detection limit in pure bacterial cultures of Erwinia carotovora

4.3 Comparison of four DNA extraction methods

4.4 Determination of the detection limit of $E c$ in potato peel extracts

4.5 Comparison of microbiological and molecular genetic methods for detection of Erwinia carotovora on or in potatoes

5 SUMMARY 


\section{LIST OF ABBREVIATIONS}

$\begin{array}{ll}\text { APS } & \text { Ammoniumpersulphate } \\ \text { bd } & \text { Bidistilled } \\ \text { bidest. } & \text { Bidestilllata (=bidistilled) } \\ { }^{\circ} \mathrm{C} & \text { Degree Celisus } \\ \text { ca. } & \text { Circa } \\ \text { cfu } & \text { Colony forming units } \\ \text { cm } & \text { Centimetre } \\ \text { Co. } & \text { Company }\end{array}$

COSY Correlation spectroscopy

cv. Cultivar

CVP Crystal violet pectate

CVPB Crystal violet pectate Bulmer

CVPD Crystal violet pectate double layer

CVPM Crystal violet pectate modified

DAS-ELISA Double antibody sandwich-enzyme-linked immunosorbent assay

Dist. Distilled

DLCVP Double layer CVP medium

DNA Deoxyribonucleic acid

DNTP Desoxynuceotide-5 - Triphosphate

DSM Deutsche Sammlung von Mikroorganismen

DTE Dithioerithrol

Eca Erwinia carotovora ssp. atroseptica

Ecc Erwinia carotovora ssp. carotovora

Ech Erwinia chrysanthemi

EDTA Ethylenediaminetetraacetic acid

Fig. $\quad$ Figure

g Gram

$\mathrm{GmbH} \quad$ Gesellschaft mit beschränkter Haftung

GSPB Göttinger Sammlung phytopathogener Bakterien

h Hour (s)

ha Hectare

IF Immunofluorescence

IFC Immunofluorescence colony staining

$\mathrm{kg} \quad$ Kilogram

LPS Lipopolysaccharides

m Metre

M Molar 


$\begin{array}{ll}\text { mA } & \text { milliampere } \\ \text { mg } & \text { milligram } \\ \text { min } & \text { minutes } \\ \text { mm } & \text { millilitre } \\ \text { mPas X s } & \text { Millimetre } \\ \mu \text { gillipascal times seconds } \\ \mu 1 & \text { Microgram } \\ \text { N } & \text { Microlitre } \\ \text { NA } & \text { North } \\ \text { NMR } & \text { Nutrient agar } \\ \text { no. } & \text { nuclear magnetic resonance } \\ \text { OD } & \text { Number } \\ \text { PAGE } & \text { Optical density } \\ \text { PCR } & \text { Polyacrylamide gel electrophoresis } \\ \text { ppm } & \text { Polymerase chain reaction } \\ \text { SDS } & \text { Sodium dodecyl sulphate } \\ \text { spp. } & \text { Species (plural) } \\ \text { ssp. } & \text { Subspecies } \\ \text { Tab. } & \text { Table } \\ \text { TBE } & \text { Tris-boric acid-EDTA } \\ \text { TE } & \text { Tris-EDTA } \\ \text { TES } & \text { Tris-EDTA-2\% SDS } \\ \text { TEMED } & \text { N,N,N',N'-Tetramethylethylenediamine } \\ \text { TOCSY } & \text { total correlation spectroscopy } \\ \text { Tris } & \text { Tris-(hydroxymethyl)-amino methane } \\ \text { TTC } & \text { 2,3,5-Triphenyltetrazolium chloride } \\ \text { v/v } & \text { Volume per volume } \\ \text { w/w } & \text { Weight per weight } \\ \text { YDC } & \text { Yeast-dextrose-chalk agar } \\ & \end{array}$


Dedicated

To my mother, my wife, our children and my late father 


\section{CHAPTER I}

\section{Detection of Erwinia carotovora in potato stocks using a semi-selective medium}

\section{INTRODUCTION}

Seed potato tubers contaminated with Erwinia carotovora subsp. atroseptica (Eca) (van Hall 1902) Dye 1969 and Erwinia carotovora subsp. carotovora (Ecc) (Jones, 1901) Bergey, Harrison, Breed, Hammer \& Huntoon, 1923, are considered the main source of primary inoculum for blackleg and soft rot of potato crops (LANGERFELD, 1984; HARJU and KANKILA, 1993; PEROMBELON and SALMOND, 1995). In addition Ecc can occur in many important horticultural crops (ALIPPI et al., 1997; CLARK et al., 1998). Both subspecies are commonly associated with potato tuber soft rot, but rot in the basal part of the stem (blackleg disease) is usually caused by Eca (PEROMBELON and KELMAN, 1980). Ecc occurs in both temperate and warm climates but mostly in storage. Since there exist no effective chemical control measures for the disease during storage or in the field, reduction in yield losses can only be achieved by following good crop husbandry, use of non-contaminated planting materials (TOTH et al., 1996) and cultivation of resistant or tolerant varietes (ROBINSON and FOSTER, 1987; DÖPKE and RUDOLPH, 1990; STEFANI et al., 1990). The rotting of mother tubers during the growing season has been reported as the major source of inoculum for contaminating progeny tubers, which later in storage, when conditions are favourable, could lead to losses due to soft rot of tubers (PEROMBELON; 1976, 1992).

A variety of different methods for detecting of the latent stage of the disease have been used by several authors (ALLAN and KELMAN, 1977; VRUGGINK and DE BOER, 1978; PEROMBELON et al., 1987). Especially, the crystal violet pectate medium (CVP) (CUPPELS \& KELMAN, 1974) and several modified selective media (PEROMBELON and BURNETT, 1991; BDLIYA, 1995) are used for detection of potato soft rotting erwinias from potatoes. The dilution plating of samples onto a semi-selective medium to isolate the bacteria has been in use for a long time. Its use has been extended to the quantification and identification of erwinias in mixed populations from plants, when characteristic deep cavities are formed only by pectolytic soft rot erwinias on the semi-selective medium (AHMED et al., 2000). Sodium polypectate, the main component of CVPB used in the original formulation, was produced by H. P. Bulmer Ltd. (PEROMBELON and BURNETT, 1991). It is no longer available and preparations from other sources have been found to be unsatisfactory because of poor gelling capacity and because typical cavities were not formed on media containing these pectin preparations. 
The purpose of this study was to develop an easy, reliable and sensitive microbiological test for quantifying the latent contamination of potato tubers by erwinias. The suitability of the assay developed was tested on a large scale in 3 vegetation periods in cooperation with Stöver Produktion $\mathrm{GmbH}$ to investigate the populations and survival of erwinias on superficially contaminated potato tubers. 


\section{MATERIAL AND METHODS}

\subsection{Media for bacteria}

All the media used in this study were sterilised by autoclaving at $121^{\circ} \mathrm{C}$ for $15 \mathrm{~min}$. The $\mathrm{pH}$ of the media was adjusted using $\mathrm{NaOH}$ or $\mathrm{HCl}$ before autoclaving. Antibiotics were sterile filtered and added to the medium after autoclaving and cooling down to about $45-50{ }^{\circ} \mathrm{C}$.

\subsubsection{Yeast- dextrose- chalk agar (YDC) (LELLIOTT and STEAD, 1987)}

$\begin{array}{lc}\text { Yeast extract (Oxoid L 21) } & 10.0 \mathrm{~g} \\ \mathrm{CaCO}_{3} & 20.0 \mathrm{~g} \\ \text { D-Glucose } & 20.0 \mathrm{~g} \\ \text { Agar } & 15.0 \mathrm{~g} \\ \text { Dist. water } & 1000.0 \mathrm{ml}\end{array}$

\subsubsection{King's medium B (KING et al., 1954)}

Proteose peptone (Oxoid L 46) $20.0 \mathrm{~g}$

$\mathrm{K}_{2} \mathrm{HPO}_{4}$ $1.5 \mathrm{~g}$

$\mathrm{MgSO}_{4}$ X $7 \mathrm{H}_{2} \mathrm{O}$

$1.5 \mathrm{~g}$

Glycerol $10.0 \mathrm{ml}$

Agar

$15.0 \mathrm{~g}$

Dist. water

$1000.0 \mathrm{ml}$

$\mathrm{pH} 7.2$

\subsubsection{Crystal violet pectate (Bulmer) medium (CVPB) (PEROMBELON and} BURNETT, 1991)
A- Cold dist.water
$500.0 \mathrm{ml}$
aqueous crystal violet solution
$1.0 \mathrm{ml}(0.075 \%(\mathrm{w} / \mathrm{v})$ solution $)$
$\mathrm{CaCl}_{2} \times 2 \mathrm{H}_{2} \mathrm{O}$
$6.8 \mathrm{ml} \mathrm{10 \%} \mathrm{(freshly} \mathrm{prepared} \mathrm{solution)}$
$\mathrm{NaNO}_{3}$
$1.0 \mathrm{~g}$
tri-Na citrate dihydrate
$2.5 \mathrm{~g}$
Agar
$2.0 \mathrm{~g}$
Tryptone (Oxoid L 42)
$0.5 \mathrm{~g}$
B- Sodium polypectate (Bulmer)
$9.0 \mathrm{~g}$ 
Add sodium polypectate slowly with constant stirring and autoclave at $121{ }^{\circ} \mathrm{C}$ for $15 \mathrm{~min}$. Cool the medium to about $45-50{ }^{\circ} \mathrm{C}$, add $2 \mathrm{ml}$ of $1 \%$ novobiocin (sodium salt, Sigma) and pour into Petri plates.

\subsubsection{Double layer CVP medium (DLCVP) (PEROMBELON and BURNETT, 1991)}

\section{Basal layer}

$\mathrm{CaCl}_{2} \times 2 \mathrm{H}_{2} \mathrm{O}$

Tryptone (Oxoid L 42)

Crystal violet solution

$\mathrm{NaNO}_{3}$

Agar

Dist. Water

$\mathrm{pH}$

\section{Overlayer}

$$
5.5 \mathrm{~g}
$$

$1.0 \mathrm{~g}$

$2.0 \mathrm{ml}$ of $0.075 \%$

$16.0 \mathrm{~g}$

$15.0 \mathrm{~g}$

$1000 \mathrm{ml}$

$7.0-7.5$
Sodium polypectate $25 \mathrm{~g}$

EDTA (disodium salt) $20 \mathrm{ml} 5 \%$

Dist. water $\quad 1000 \mathrm{ml}$

$\mathrm{pH}$

Before pouring, the basal layer was melted, supplemented with $40 \mu \mathrm{g} / \mathrm{ml}$ novobiocin, and 15 $\mathrm{ml}$ were dispensed into each Petri plate and allowed to set before pouring the $5 \mathrm{ml}$ overlayer in each Petri plate.

\subsubsection{Crystal violet pectate double layer medium (BDLIYA, 1995)}

The semi-selective medium (CVPD) for isolation of pectolytic Erwinia spp. consists of two layers as follows :

\section{Basal layer}

Peptone $\quad 2.0 \mathrm{~g}$

Yeast extract $0.6 \mathrm{~g}$

$\mathrm{NaCl} \quad 1.0 \mathrm{~g}$

Agar $\quad 3.0 \mathrm{~g}$

Tryptone $\quad 1.5 \mathrm{~g}$

L-Asparagine $0.5 \mathrm{~g}$

SDS $\quad 50 \mathrm{mg}$

Dist. water $250 \mathrm{ml}$

\section{$\underline{\text { Overlayer }}$}

Crystal violet solution

$1.0 \mathrm{ml}, 0.075 \%(\mathrm{w} / \mathrm{v})$

$\mathrm{KNO}_{3}$

$1.0 \mathrm{~g}$

tri-Na citrate dihydrate

$2.5 \mathrm{~g}$

Agar

$2.0 \mathrm{~g}$

Tryptone

$1.5 \mathrm{~g}$

$\mathrm{CaCl}_{2} \times 2 \mathrm{H}_{2} \mathrm{O}$

$6.8 \mathrm{ml}$ (10\% solution)

Bromothymol blue

$0.5 \mathrm{ml}(0.5 \%$ solution $)$

Cold dist. water $500.0 \mathrm{ml}$

Sodium polypectate "Bulmer" 1) $9.0 \mathrm{~g}$

1) H. P. Bulmer Ltd., Plough Lane, Hereford HR4 OLE, England.

Add $0.5 \mathrm{ml}$ of filter sterilised $1 \%$ solution of 2,3,5-triphenyltetrazolium chloride to the basal medium after autoclaving and cooling down to $50^{\circ} \mathrm{C}$, and add $3.2 \mu \mathrm{g} / \mathrm{ml}$ Polymxin B sulphate solution. Dispense $12 \mathrm{ml}$ of basal medium per plate and allow to set for $10 \mathrm{~min}$. Then add 1.2 
$\mu \mathrm{g} / \mathrm{ml}$ Polymxin B sulphate to the overlayer solution after autoclaving and cooling down to 45 ${ }^{\circ} \mathrm{C}$, and pour $15 \mathrm{ml} /$ plate on top of the basal medium.

\subsection{6 $\quad \alpha$-Methyl-d-glucoside medium (SAETTLER et al., 1989)}

The medium is made up of 5 parts
A- $\mathrm{KH}_{2} \mathrm{PO}_{4}$
$2.0 \mathrm{~g}$
$\mathrm{K}_{2} \mathrm{HPO}_{4}$
$7.0 \mathrm{~g}$
$\mathrm{NH}_{4} \mathrm{Cl}$
$1.0 \mathrm{~g}$
Dist. Water
$500.0 \mathrm{ml}$
B- Agar
$15.0 \mathrm{~g}$
Casamino acids (Difco)
$1.0 \mathrm{~g}$
Dist. water $500.0 \mathrm{ml}$
C- $\mathrm{MgSO}_{4} \mathrm{X} 7 \mathrm{H}_{2} \mathrm{O}$
$1.0 \mathrm{ml}$ (10\% solution)
D- $\alpha$-Methyl-d-glucoside

(Sigma M 9376)

$50 \quad \mathrm{ml}$ (20\% solution)

E - 2,3,5-Triphenyl-tetrazoliumchloride $2.0 \mathrm{ml}$ (1\% solution)

Each solution of the 5 parts is autoclaved separately. Mix A and B, cool down to $50{ }^{\circ} \mathrm{C}$, add solutions C, D and E, and pour into Petri plates.

\subsubsection{Phosphatase detection medium (SAETTLER et al., 1989)}

Bacto peptone (Difco)

$$
\begin{array}{rl}
10.0 & \mathrm{~g} \\
5.0 & \mathrm{~g} \\
15.0 & \mathrm{~g} \\
1000.0 & \mathrm{ml}
\end{array}
$$

Beef extract

Agar

Dist. water

pH 7.0

\subsubsection{Medium for indole production (SAETTLER et al., 1989)}

Tryptone (Difco)

$$
\begin{array}{rl}
10.0 & \mathrm{~g} \\
1.0 & \mathrm{~g}
\end{array}
$$

L-Tryptophane

Dist. water

$$
1000.0 \mathrm{ml}
$$

\subsubsection{Kovac's indole reagent (LELLIOTT and STEAD, 1987)}

$p$ - Dimethylaminobenzaldehyde

$5.0 \mathrm{~g}$

Amyl alcohol

$75.0 \mathrm{ml}$

conc. $\mathrm{HCl}$

$25.0 \mathrm{ml}$ 
Dissolve the aldehyde in the alcohol by heating gently in a water bath at $50{ }^{\circ} \mathrm{C}$. Cool down and add the acid to give a straw-brown coloured liquid. Store in a dark glass bottle at $4{ }^{\circ} \mathrm{C}$.

\subsubsection{Nutrient agar (NA) (LELLIOTT and STEAD, 1987)}

Beef extract

$1.0 \mathrm{~g}$

Peptone (Oxoid L34)

$5.0 \mathrm{~g}$

Yeast extract (Oxoid L 21)

$2.0 \mathrm{~g}$

Agar (Oxoid No.3)

$15.0 \mathrm{~g}$

$\mathrm{NaCl}$

$5.0 \mathrm{~g}$

Dist. water

$1000.0 \mathrm{ml}$

\subsubsection{Gas production from glucose (KELMAN and DICKEY, 1980)}
A- Bacto peptone (Difco)
$10.0 \mathrm{~g}$
Bromocresol purpur
$0.7 \mathrm{ml} 1.5 \%$ solution
Dist.water $1000.0 \mathrm{ml}$
B- Glucose
$10.0 \mathrm{~g}$
Dist. water
$1000.0 \mathrm{ml}$

Place one Durham-tube upside down into each test tube filled with $4.5 \mathrm{ml}$ of solution A and autoclave. Filter sterilise solution B and add $0.5 \mathrm{ml}$ to each test tube after it has been cooled down to about $45-50{ }^{\circ} \mathrm{C}$.

\subsubsection{Logan's medium (FAHY and HAYWARD, 1983)}

Nutrient agar $28.0 \mathrm{~g}$

Yeast extract

Glucose

$5.0 \mathrm{~g}$

Dist. water $1000.0 \mathrm{ml}$

After autoclaving and cooling to about $45-50{ }^{\circ} \mathrm{C}$ the medium is poured into plates.

\subsubsection{Lecithinase activity (Egg yolk test)}

Egg yolk emulsion was prepared from a fresh hen egg, which was washed and sterilized in $70 \%$ ethanol for $5 \mathrm{~min}$. The egg was flamed, broken aseptically, and the yolk separated into a sterile measuring cylinder and diluted to $1.5 \%$ (v/ v) with sterile water. $100 \mathrm{ml}$ of this egg yolk emulsion were added to $900 \mathrm{ml}$ of molten Nutrient agar (NA) (see 2.1.8) medium, cooled to $55^{\circ} \mathrm{C}$ and poured into Petri plates. 


\subsubsection{Salt tolerance}

Salt tolerance was determined by inoculating the bacterial strains into Nutrient broth as described in 2.1.8, but without agar. $\mathrm{NaCl}$ was added to the liquid medium to obtain the required final concentration of $5 \% \mathrm{NaCl}$. After autoclaving and cooling to about $45-50{ }^{\circ} \mathrm{C}$ the medium was poured into plates.

\subsubsection{Mineral salt-pectin-medium (QUANTICK et al., 1983)}

\section{Solution A :}

$\mathrm{KH}_{2} \mathrm{PO}_{4}$

$\mathrm{Na}_{2} \mathrm{HPO}_{4} \mathrm{X} \mathrm{H}_{2} \mathrm{O}$

Dist. water $200 \mathrm{ml}$

pH 6.9

\section{Solution B:}

$\left(\mathrm{NH}_{4}\right)_{2} \mathrm{SO}_{4}$

$2.4 \mathrm{~g}$

$\mathrm{MgSO}_{4} \times 7 \mathrm{H}_{2} \mathrm{O} \quad 0.5 \mathrm{~g}$

$\mathrm{H}_{3} \mathrm{BO}_{3} \quad 0.6 \mathrm{mg}$

$\mathrm{CuSO}_{4}$ X $5 \mathrm{H}_{2} \mathrm{O} \quad 1.2 \mathrm{mg}$

$\mathrm{MnSO}_{4} \mathrm{X} \mathrm{H}_{2} \mathrm{O} \quad 1.2 \mathrm{mg}$

$\mathrm{Na}_{2} \mathrm{MoO}_{4} \mathrm{X} 2 \mathrm{H}_{2} \mathrm{O} \quad 1.2 \mathrm{mg}$

$\mathrm{CaCl}_{2} \quad 12 \mathrm{mg}$

$\mathrm{ZnSO}_{4} \times 7 \mathrm{H}_{2} \mathrm{O} \quad 12 \mathrm{mg}$

Dist. Water $100 \mathrm{ml}$

Solution C:

$\mathrm{FeCl}_{3} \times 6 \mathrm{H}_{2} \mathrm{O} \quad 12 \mathrm{mg}$ (sterile filtrate)

Solution D:

Dissolve $6 \mathrm{~g}$ pectin $\mathrm{N}$ in $300 \mathrm{ml}$ water and boil daily one hour at $100{ }^{\circ} \mathrm{C}$ for 3 days. Solutions $\mathrm{A}$ and $\mathrm{B}$ are autoclaved separately and then mixed with solution $\mathrm{C}$ after cooling to about 45-50 ${ }^{\circ} \mathrm{C}$. Solution $\mathrm{D}$ is added by mixing, and the resulting mixture is divided into portions of $50 \mathrm{ml}$ in $100 \mathrm{ml}$ flasks and stored at $4{ }^{\circ} \mathrm{C}$.

\subsubsection{Antibiotics used in this study}

Novobiocin (mono Na-salt)( Sigma chemicals)

Polymyxin B sulphate (Sigma chemicals, USA)

Rifampicin (Merck)

Streptomycin sulphate (Sigma chemicals) 


\subsection{Characterization of Erwinia spp.}

\subsubsection{Bacterial strains used in these studies}

Table 1: Origin and source of bacterial strains used in this study

\begin{tabular}{|l|l|l|}
\hline Strain number & Species/ subspecies & Origin \\
\hline 1401 & Eca (Erwinia carotovora ssp. atroseptica) & GSPB \\
\hline 2231 & Eca (Erwinia carotovora ssp. atroseptica) & GSPB \\
\hline 9201 & Eca (Erwinia carotovora ssp. atroseptica) & $\left.\mathrm{DSM}^{2}\right)$ \\
\hline 9204 & Eca (Erwinia carotovora ssp. atroseptica) & $\mathrm{DSM}$ \\
\hline 2967 & Eca (Erwinia carotovora ssp. atroseptica) & $\mathrm{DSM}$ \\
\hline 426 & Ecc (Erwinia carotovora ssp. carotovora) & $\mathrm{GSPB}$ \\
\hline 427 & Ecc (Erwinia carotovora ssp. carotovora) & $\mathrm{GSPB}$ \\
\hline 429 & Ecc (Erwinia carotovora ssp. carotovora) & $\mathrm{GSPB}$ \\
\hline 436 & Ecc (Erwinia carotovora ssp. carotovora) & $\mathrm{GSPB}$ \\
\hline 8201 & Ecc (Erwinia carotovora ssp. carotovora) & $\mathrm{DSM}$ \\
\hline 8202 & Ecc (Erwinia carotovora ssp. carotovora) & $\mathrm{DSM}$ \\
\hline 8203 & Ecc (Erwinia carotovora ssp. carotovora) & $\mathrm{DSM}$ \\
\hline Strains A ${ }^{3)}$ and & $\begin{array}{l}\text { Eca (Erwinia carotovora ssp. atroseptica) } \\
\text { Strains B }\end{array}$ & Field experiments \\
\hline Eca 2962 & Eca (Erwinia carotovora ssp. carotovora) & \\
\hline
\end{tabular}

1) GSPB = Göttinger Sammlung phytopathogener Bakterien, Göttingen, Germany

2) $\mathrm{DSM}=$ Deutsche Sammlung von Mikroorganismen, Braunschweig, Germany

3) = Strains A and B were isolated in these studies from field experiments 2000 and lyophilized under the GSPB numbers of 3050, 3051 and 3052 for Eca and 3053, 3054 and 3054 for $E c c$.

4) Strain obtained from Dr. Fukuoka, Shikoku National Industrial Research Institute, Takamatsu, Japan

\subsubsection{Isolation of Erwinia spp. from different potato specimens}

Sixteen different potato tubers specimens (see 3.5.2) without disease symptoms were peeled with a peelstrip (ca. $2 \mathrm{~mm}$ thick) by hand. The peels of each specimen were crushed individually in a sterile mortar and pestle with $4 \mathrm{ml} 0.01 \mathrm{M}$ magnesium sulphate buffer and allowed to settle at room temperature for $5 \mathrm{~min}$, after which a serial 1:10 dilution was carried out in seven steps. From each dilution $0.1 \mathrm{ml}$ were pipetted into a Petri dish with the semi- 
selective medium and evenly distributed with a Drigalsky spatula. Three plates per each dilution were incubated at $27^{\circ} \mathrm{C}$ for $24 \mathrm{~h}$. Those bacteria which had caused colonies with deep cavities within one day were tranferred with a toothpick onto King's medium B and incubated at $27^{\circ} \mathrm{C}$ for $24 \mathrm{~h}$. The isolates obtained were characterized on the basis of the following tests: utilisation of $\alpha$-methyl-d-glucoside, phosphatase activity, growth at different temperatures, indole production, gas production from glucose, growth on Logan's medium, and rotting of potato tuber slices.

\subsubsection{Conventional physiological tests}

\subsubsection{Utilisation of $\alpha$-methyl-d-glucoside}

The bacterial isolates to be tested were precultured on plates with King's B medium at $27^{\circ} \mathrm{C}$ for $24 \mathrm{~h}$, transferred onto the $\alpha$-methyl-d-glucoside medium (see 2.1.3) and incubated at $27^{\circ} \mathrm{C}$ for $48 \mathrm{~h}$. A positive reaction was indicated by a pink colour of the colony. Only strains of Erwinia carotovora subsp. atroseptica (Eca) show a positive reaction, but not those of Erwinia carotovora subsp. carotovora (Ecc) and Erwinia chrysanthemi (Ech).

\subsubsection{Phosphatase production}

The bacterial isolates to be tested were precultured on plates with King's B medium at $27^{\circ} \mathrm{C}$ for $24 \mathrm{~h}$, spot-inoculated onto the phosphatase detection medium (see 2.1.4) and incubated at $27{ }^{\circ} \mathrm{C}$ for $48 \mathrm{~h}$. For visualisation of phosphatase activity $1.0 \mathrm{ml}$ of ammonium solution (33\%) (KELMAN and DICKEY, 1980) were placed on the open lid of each Petri dish and the plate culture inverted over it. Plates were evaluated after $10 \mathrm{sec}$. Only strains of Ech show a positive reaction for phosphatase.

\subsubsection{Differentiation of Erwinia spp. by growth at different temperatures}

The bacterial isolates were streaked onto plates with King's B medium and incubated at three different temperatures $\left(27^{\circ} \mathrm{C}, 35.5^{\circ} \mathrm{C}\right.$ and $\left.37^{\circ} \mathrm{C}\right)$ for $24 \mathrm{~h}$. Triplicate plates were evaluated at each temperature. Ech is able to grow and form characteristic cavities at all three temperatures, Ecc does so at $27{ }^{\circ} \mathrm{C}$ and $35.5^{\circ} \mathrm{C}$ only, and Eca only at $27{ }^{\circ} \mathrm{C}$ (JANSE and SPIT, 1989; PEROMBELON and HYMAN, 1986). 


\subsubsection{Indole production}

The bacterial isolates were inoculated into the indole production medium (see 2.1.6) by adding $0.1 \mathrm{ml}$ of a bacterial suspension in $0.01 \mathrm{M} \mathrm{MgSO}_{4}$ from fresh bacterial cultures adjusted to an O.D 660 of 0.06 (ca. $\left.10^{8} \mathrm{cfu} / \mathrm{ml}\right)$ to $5 \mathrm{ml}$ medium. The tubes were incubated at $27{ }^{\circ} \mathrm{C}$ for $48 \mathrm{~h}$. After incubation, $0.5 \mathrm{ml}$ of Kovac's reagent (see 2.1.11) were added to $5 \mathrm{ml}$ of the culture medium and shaken gently. A positive reaction was indicated by a dark red colour. Only strains of Ech show a positive reaction (LELLIOTT and STEAD, 1987).

\subsubsection{Gas production from glucose}

The bacterial isolates were inoculated into test tubes with the gas production medium (see 2.1.8) by transferring $0.1 \mathrm{ml}$ of a bacterial suspension containing $10^{8} \mathrm{cfu} / \mathrm{ml}$ and incubating at $27{ }^{\circ} \mathrm{C}$ for 2-7 days. After incubation only strains of Ech show gas production in the Durham tubes (KELMAN and DICKEY, 1980).

\subsubsection{Logan's medium}

The bacterial isolates were transferred onto Logan's medium (see 2.1.9) by spot inoculation with $0.1 \mathrm{ml}$ bacterial suspension of $10^{8} \mathrm{cfu} / \mathrm{ml}$, and incubated at $27{ }^{\circ} \mathrm{C}$ for $24 \mathrm{~h}$. After incubation Eca shows small colonies with a red centre, Ecc big light red colonies and Ech dark red colonies.

\subsubsection{Lecithinase activity (Egg yolk test)}

The bacterial isolates to be tested were precultured on plates with King's medium B at $27{ }^{\circ} \mathrm{C}$ for $24 \mathrm{~h}$, streaked onto egg yolk medium (see 2.1.11) and incubated at $27{ }^{\circ} \mathrm{C}$ for 7 days. After incubation colonies of $E c h$ are surrounded by a highly turbid zone due to lecithinase activity.

\subsubsection{Salt tolerance}

Tolerance to $5 \% \mathrm{NaCl}$ was detected by visible turbidity after the bacterial isolates were inoculated into test tubes with the salt tolerance $(5 \% \mathrm{NaCl})$ medium (see 2.1.12) by transferring $0.1 \mathrm{ml}$ of a bacterial suspension containing $10^{8} \mathrm{cfu} / \mathrm{ml}$ and incubating at $27^{\circ} \mathrm{C}$ for 14 days. After incubation only strains of Ech show a visible turbidity. 


\subsubsection{Biotest on potato tuber slices}

The bacterial isolates were grown onto King' s medium B for $24 \mathrm{~h}$. Potato tubers, cultivar Cilena, were washed and sterilised with $96 \%$ ethanol and flaming, cut with a sterile knife to slices of 8-10 mm and placed into Petri dishes. Ten milliliter of 0.01 M magnesium sulphate were added to each Petri dish, and each slice was inoculated with a drop of $10 \mu 1$ of $10^{8} \mathrm{cfu} / \mathrm{ml}$ of bacteria to be tested and incubated at $27^{\circ} \mathrm{C}$ for $24 \mathrm{~h}$. Each bacterial strain was tested threefold. A positive reaction was recorded when the potato slices showed soft rotting within two days.

\subsubsection{Maintenance and storage of bacterial cultures}

The working strains were maintained in sterile distilled water at room temperature in Eppendorf tubes, some of the strains were maintained on YDC agar slants in test tubes and stored at $4{ }^{\circ} \mathrm{C}$. For long time storage the strains were preserved as lyophilized cultures.

\subsection{Development of a new semi-selective medium for $E$. carotovora}

2.3.1 Effect of tryptone in the basal and overlayer on the growth of Erwinia spp. on the semi-selective medium

The effect of different concentrations of tryptone $(0,0.3 \%, 0.6 \%)$ in the basal and overlayer on the growth of Erwinia spp. was investigated to determine the growth and cavity formation by Erwinia spp.. The bacterial strains Eca 1401 and Ecc 1405 were grown on King's medium B for $24 \mathrm{~h}$, adjusted to an O.D. 660 of 0.06 (ca. $8 \times 10^{7} \mathrm{cfu} / \mathrm{ml}$ ), and serially diluted. $0.1 \mathrm{ml}$ of the bacterial suspensions from the dilution $1: 10^{6}$ were plated on the CVPM medium and incubated at $27^{\circ} \mathrm{C}$ for $24 \mathrm{~h}$ (see table 8 ).

\subsubsection{Effect of tri-sodium citrate dihydrate in the basal and over layer on the growth of Erwinia spp. on the semi-selective medium}

The effect of tri-sodium citrate dihydrate was studied by addition of different concentrations ( 0 , $0.1 \%, 0.2 \%, 0.3 \%$ and $0.4 \%$ ) to both the basal and overlayer, and the bacterial suspensions were plated on the medium as described above. 


\subsubsection{Comparison of different pectin sources for isolation of Erwinia spp. on the semi-selective medium}

The bacterial strains were grown on King's medium B and incubated at $27{ }^{\circ} \mathrm{C}$ for $24 \mathrm{~h}$. Bacterial suspensions were adjusted to an $\mathrm{OD}_{660}$ of $0.06\left(10^{8} \mathrm{cfu} / \mathrm{ml}\right)$, and $0.1 \mathrm{ml}$ were plated on the Petri dishes with the following different pectin sources:

Copenhagen pectin A/S (Hercules Slendid ${ }^{\circledR}$ type 440$)$, is a lowly esterified pectin $(<10 \%)$ extracted from citrus peel

pectin A (Roth Co. no. 9123.1) extracted from apple, medium degree of esterification (ca. $36 \%)$

pectin C (Roth Co. no. 8911.1), extracted from citrus, high degree of esterification (ca. 67$71 \%)$

pectin N (Roth Co. no. 8913.1) pectic acid sodium salt with low degree of esterification $(8.0 \%)$;

pectin from citrus fruits (Sigma no. 9135), galacturonic acid content 80\%, methoxy content $9 \%)$

pectin from apple (ICN Biomedicals no. 156057), polygalacutronic acid methyl ester);

pectin from citrus fruits (ICN Biomedicals no. 102587), polygalacutronic acid methyl ester);

pectin from citrus (Serva no. 31650), high degree of esterification (ca. 65\%);

pectin from citrus peel (Fluka Co. no. 76280), high degree of esterification (63-66\%);

polygalacturonic acid (Sigma no. P- 3850) from citrus fruit;

sodium polypectate (Sigma no. P- 1879), sodium salt, minimum 85\%;

sodium polypectate "Bulmer” (H. P. Bulmer Ltd., Plough Lane, Hereford HR4 OLE, England); pectic acid from citrus (Herbstreith \& Fox CU-L 023/00), low degree of esterification (7\%), lot no. 006645 ;

pectic acid from apple (Herbstreith \& Fox AU-L 011/01), low degree of esterification (5\%), lot no. 102576;

pectin from apple (Herbstreith \& Fox AU-L 012/01), high degree of esterification (32\%), lot no. 102577 ;

(see also table 5) and incubated at $27^{\circ} \mathrm{C}$ for $24 \mathrm{~h}$. 


\subsubsection{Evaluation of bacterial growth on the semi-selective medium}

Evaluation was based on growth $(++=$ bacteria grew on the medium, - = no growth), and cavity formation was evaluated by a scale of $0-3(0=$ no cavity, $1=$ shallow cavity, $2=$ moderately deep and wide, and 3 = deep and wide cavity).

\subsubsection{Recovery of Erwinia spp. from artificially inoculated potato extracts on semi-selective media and on King s medium B}

Potato tubers without disease symptoms was peeled with a peelstrip ca. $2 \mathrm{~mm}$ thick by hand. The peel was weighed and $1 \mathrm{~g}$ was crushed in a sterile mortar and pestle with $4 \mathrm{ml} 0.01 \mathrm{M}$ magnesium sulphate solution and allowed to settle at room temperature for $5 \mathrm{~min}$. The bacterial strain Ecc 436 (Erwinia carotovora ssp. carotovora) was grown on Petri dishes with King' s medium B and incubated at $27^{\circ} \mathrm{C}$ for $24 \mathrm{~h}$. The bacterial growth was scraped off and suspended in 0.01 M magnesium sulphate solution. The bacterial suspension was adjusted to an $\mathrm{OD}_{660}$ of 0.06 (ca. $10^{8} \mathrm{cfu} / \mathrm{ml}$ ), and $0.1 \mathrm{ml}$ were mixed with potato peel homogenate. The mixture was serially diluted, and from each dilution $0.1 \mathrm{ml}$ were pipetted into a Petri dish and evenly distributed with a Drigalsky spatula. Three plates per each dilution were incubated at 27 ${ }^{\circ} \mathrm{C}$ for $24 \mathrm{~h}$.

\subsubsection{Detection limit of Erwinia spp. on the semi-selective medium and on King' s medium B}

The same method as described above (2.3.5) was used, but suspensions with lower concentrations of bacteria $\left(10^{4}\right.$ and $\left.10^{3} \mathrm{cfu} / \mathrm{ml}\right)$ were added to the potato homogenates, serially diluted, and $0.1 \mathrm{ml}$ were plated on both, CVPM and King's medium B, and incubated at $27^{\circ} \mathrm{C}$ for $24 \mathrm{~h}$.

\subsubsection{Isolation of Erwinia spp. on the new semi-selective medium}

\subsubsection{Potato peel extracts}

Twenty healthy potato tubers were peeled by a hand-peeler, ca. $2 \mathrm{~mm}$ thick and weighed. The peels were comminuted in a double amount of $0.01 \mathrm{M}$ magnesium sulphate solution by a homogenizer (Minipimer contro plus, vario, Braun, Melsungen, Germany). The homogenate was allowed to settle at room temperature for $5 \mathrm{~min}$, serially diluted, and $0.1 \mathrm{ml}$ were plated on the CVPM medium and incubated at $27{ }^{\circ} \mathrm{C}$ for $24 \mathrm{~h}$. Triplicate plates were used for each experiment. 


\subsubsection{Soakage of potato tubers}

Twenty potato tubers per sample were soaked in $0.01 \mathrm{M}$ magnesium sulphate for $3 \mathrm{~h}$ at $4{ }^{\circ} \mathrm{C}$ with occasional stirring every $30 \mathrm{~min}$ by hand. After incubation, $50 \mathrm{ml}$ from each soakage were centrifuged at $5000 \mathrm{~g}$ for $15 \mathrm{~min}$. The pellet was dissolved in $1 \mathrm{ml} 0.01 \mathrm{M}$ magnesium sulphate and serially diluted $1: 10$ and 1:100. From each dilution $0.1 \mathrm{ml}$ were plated on the CVPM medium and incubated at $27^{\circ} \mathrm{C}$ for $24 \mathrm{~h}$.

\subsection{Evaluation of latent contamination of 232 potato tuber samples from the years 1998, 1999 and 2000}

In the three years 1998, 1999 and 2000232 potato tuber samples were obtained from Stöver Co. for detection of latent contamination by erwinias. Each sample consisted of 60 tubers. The samples were stored at $4{ }^{\circ} \mathrm{C}$ and analysed within 4 weeks. Each sample was divided in three groups of 20 tubers each and analysed separately ("experiments" 1, 2 and 3, see tables 15, 16 and 17) according to the method described in 2.3.7.2.

\section{$2.5 \quad$ Field experiments}

The field experiments were performed by Stöver Co. on a farm in 27239 Natenstedt near Twistringen to compare potato lots with different degrees of latent contamination as determined in Göttingen. Soil type: (h) $\mathrm{SL}, \mathrm{pH}: 5.7, \mathrm{mg}_{2} \mathrm{O}_{5}: 26$ (D), $\mathrm{mg} \mathrm{K}_{2} \mathrm{O}: 23$ (D), mg $\mathrm{Mg}: 5$ (B), N-min : 0-30 cm $119 \mathrm{~kg} \mathrm{NO}_{3}-\mathrm{N} / \mathrm{ha}, \mathrm{N}-\mathrm{min}: 30-60 \mathrm{~cm} 33 \mathrm{~kg} \mathrm{NO}{ }_{3}-\mathrm{N} / \mathrm{ha}$. Potatoes were planted on April 4, 1999 by hand. The soil was dry and warm, temperatures $16{ }^{\circ} \mathrm{C}$. Each variant consisted of 4 blocks, each with two rows of 24 potatoes totally, planted with a space of $37 \mathrm{~cm}$. The usual plant protection measures were performed, including weekly sprays against Phytophthora infestans. The summer 1999 was unusually warm with high soil temperatures and rainfall below the yearly average. The potatoes were harvested on September 20, 1999, by hand. Evaluation in each block included : Total number of potato tubers, numbers of tubers with soft rot, total weight of tubers, weight of fractions according to tuber sizes, number of tubers in different fractions per $10 \mathrm{~kg}$. 


\section{$3 \quad$ RESULTS}

\subsection{Preservation of the Erwinia spp. used in this study}

Three different methods were compared for preservation of viable cultures of Erwinia spp.. Lyophilization appeared to be most effective in maintaining the bacterial cultures without losing viability. On the other hand, this method needs special apparates (lyophilizator, ampoule-constrictor) and is time-consuming.

Storage of the cultures on YDC medium slants at $4{ }^{\circ} \mathrm{C}$ maintained viability of the $E c c$ cultures for at least 6 months and that of Eca for 3 - 4 weeks, whereas the cultures of Ech died within 34 weeks. On the other hand, storage of Erwinia spp., Eca and Ecc in sterile distilled water at room temperature maintained the viability of all Erwinia spp. for more than 16 months, at $4{ }^{\circ} \mathrm{C}$ as well as at room temperature (Tables 2 and 3). Therfore, most of the Erwinia strains used in this study were preserved in sterile distilled water at room temperature .

Table 2 : Evaluation of different preservation methods for maintaining the viability of Erwinia spp. stored at $4^{\circ} \mathbf{C}$. Eca (2967, 2231 and 1401), Ecc (426, 429 and 436), Ech (1229, 1860 and 2236)

\begin{tabular}{|c|c|c|c|c|c|c|c|c|c|c|c|c|}
\hline \multirow[t]{2}{*}{$\begin{array}{l}\text { Preservation } \\
\text { method }\end{array}$} & \multicolumn{3}{|c|}{$\begin{array}{l}\text { Viability after } \\
3-4 \text { weeks }\end{array}$} & \multicolumn{3}{|c|}{$\begin{array}{l}\text { Viability after } 6 \\
\text { months }\end{array}$} & \multicolumn{3}{|c|}{$\begin{array}{l}\text { Viability after } \\
12 \text { months }\end{array}$} & \multicolumn{3}{|c|}{$\begin{array}{l}\text { Viability after } \\
16 \text { months }\end{array}$} \\
\hline & $E c a$ & $E c c$ & Ech & Eca & Ecc & Ech & $E c a$ & $E c c$ & Ech & $E c a$ & $E c c$ & Ech \\
\hline Slants on YDC & ++ & ++ & - & - & ++ & - & - & - & - & - & - & - \\
\hline Sterile water & ++ & ++ & ++ & ++ & ++ & ++ & ++ & ++ & ++ & ++ & ++ & ++ \\
\hline
\end{tabular}

Table 3: Evaluation of different preservation methods for maintaining the viability of

Erwinia spp. stored at room temperature, the same strains were tested as in table 2.

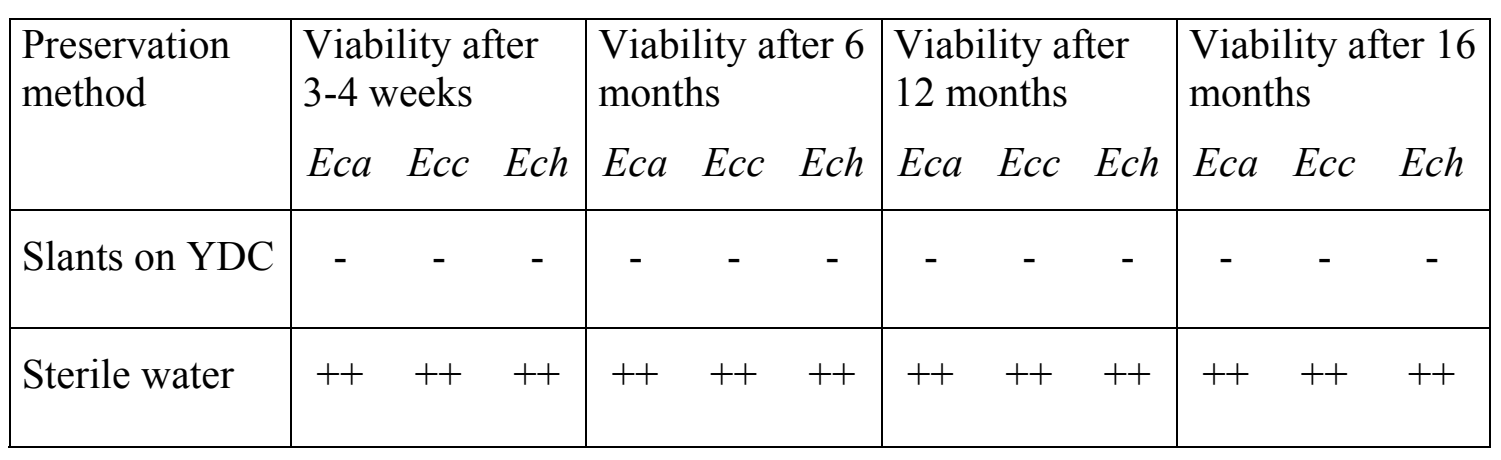

$++=$ all cultures were viable

- $\quad=$ all cultures tested were dead 


\subsection{Development of a semi-selective medium for isolation}

\section{of Erwinia spp.}

\subsubsection{Effect of tryptone and tri-sodium citrate dihydrate in the basal and} overlayer on the growth of Erwinia spp. on the semi-selective medium

In these experiments the growth on the optimal but non-selective medium King's B was compared with the semi-selective medium to which different concentrations of tryptone and citrate were added in the basal and over layer. The results obtained (Table 4) show that without tryptone and citrate bacterial colonies with cavities did not develop on the semi-selective medium, and only few colonies and weak cavities were formed when tryptone and the trisodium citrate dihydrate were added at low concentrations to the basal and over layer, respectively. On the other hand, many colonies grew when tryptone $(0.6 \%)$ was added only to the basal layer and tri-sodium citrate dihydrate $(0.4 \%)$ only to the overlayer. The addition of tryptone to the overlayer and of tri-sodium citrate dihydrate to the basal layer was not necessary.

Table 4 : Growth of bacterial strains (Eca and Ecc) on the semi-selective medium with different concentrations of tryptone and tri-sodium citrate dihydrate

\begin{tabular}{|l|c|c|}
\hline \multirow{2}{*}{ Medium } & \multicolumn{2}{|c|}{ Colonies/ Petri plate } \\
\cline { 2 - 3 } & Eca 1401 & Ecc 1405 \\
\hline King' s medium B & 8 & 9 \\
\hline without tryptone in basal layer & 0 & 0 \\
\hline without tryptone in overlayer & 0 & 4 \\
\hline $0.3 \%$ tryptone in basal layer & 3 & 5 \\
\hline $0.3 \%$ tryptone in overlayer & 4 & 8 \\
\hline $0.6 \%$ tryptone in basal layer & 8 & 3 \\
\hline $0.6 \%$ tryptone in overlayer & 4 & 1 \\
\hline without Na-citrate in basal layer & 0 & 0 \\
\hline without Na-citrate in overlayer & 0 & 0 \\
\hline $0.1 \%$ Na-citrate in basal layer & 1 & 2 \\
\hline $0.1 \%$ Na-citrate in overlayer & 2 & 1 \\
\hline $0.2 \%$ Na-citrate in basal layer & 0 & 3 \\
\hline $0.2 \%$ Na- citrate in overlayer & 4 & 0 \\
\hline $0.3 \%$ Na-citrate in basal layer & 0 & 6 \\
\hline $0.3 \%$ Na-citrate in overlayer & 7 & 2 \\
\hline $0.4 \%$ Na-citrate in basal layer & 1 & 9 \\
\hline $0.4 \%$ Na-citrate in overlayer & 8 & \\
\hline
\end{tabular}




\subsubsection{Effect of different pectin preparations on the growth of Erwinia spp.}

Table 5 and Fig. 1 clearly show that the sodium polypectate from Bulmer Co. was best suited for detection of Erwinia carotovora in comparison to 14 other different pectins. Unfortunately, the sodium polypectate from Bulmer (Plough Lane, Hereford HR4 OLE, England) is no longer available and most of the preparations from other sources are unsatisfactory because of poor gelling capacity. Only Copenhagen pectin A/S, pectin N from Roth Co., sodium polypectate from Sigma Co., and two pectin preparations from Herbstreith and Fox were found to be sastisfactory in these experiments.

Table 5 : Detection of Erwinia spp. on the CVPM medium containing 15 different pectin sources

\begin{tabular}{|l|l|c|}
\hline Pectin preparation & Source of pectin & 2 \\
\hline 1- Copenhagen pectin A/S & Hercules Slendid® type 440 & 0 \\
\hline 2- Pectin A & Roth Nr. 9123.1 & 0 \\
\hline 3- Pectin C & Roth Nr. 8911.1 & 1 \\
\hline 4- Pectin N & Roth Nr. 8913.1 & 0 \\
\hline 5- Pectin from citrus fruits & Sigma P-9135 & 0 \\
\hline 6- Pectin from apple & ICN Biomedicals Nr. 156057 & 0 \\
\hline 7- Pectin from citrus & ICN BiomedicalsNr.102587 & 0 \\
\hline 8- Pectin from citrus & Serva no. 31650 & 0 \\
\hline 9- Pectin & Fluka no. 76280 & 0 \\
\hline 10- Polygalacturonic acid & Sigma P-3850 & 2 \\
\hline 11- Sodium polypectate & Sigma P-1879 & 3 \\
\hline 12- Sodium polypectate & Bulmer & 2 \\
\hline 13- Pectic acid from citrus & Herbstreith \& Fox (CU-L 023/00) & 2 \\
\hline 14- Pectic acid from apple & Herbstreith \& Fox (AU-L 011/01) & 0 \\
\hline 15- Pectin from apple & Herbstreith \& Fox (AU-L 012/01) & \\
\hline
\end{tabular}

*) $1=$ the cavities were shallow and very small and visible after $24 \mathrm{~h}$.

$2=$ the cavities were moderately deep and wide and visible after $24 \mathrm{~h}$.

$3=$ the cavities were deep and large and were formed after $13 \mathrm{~h}$. 


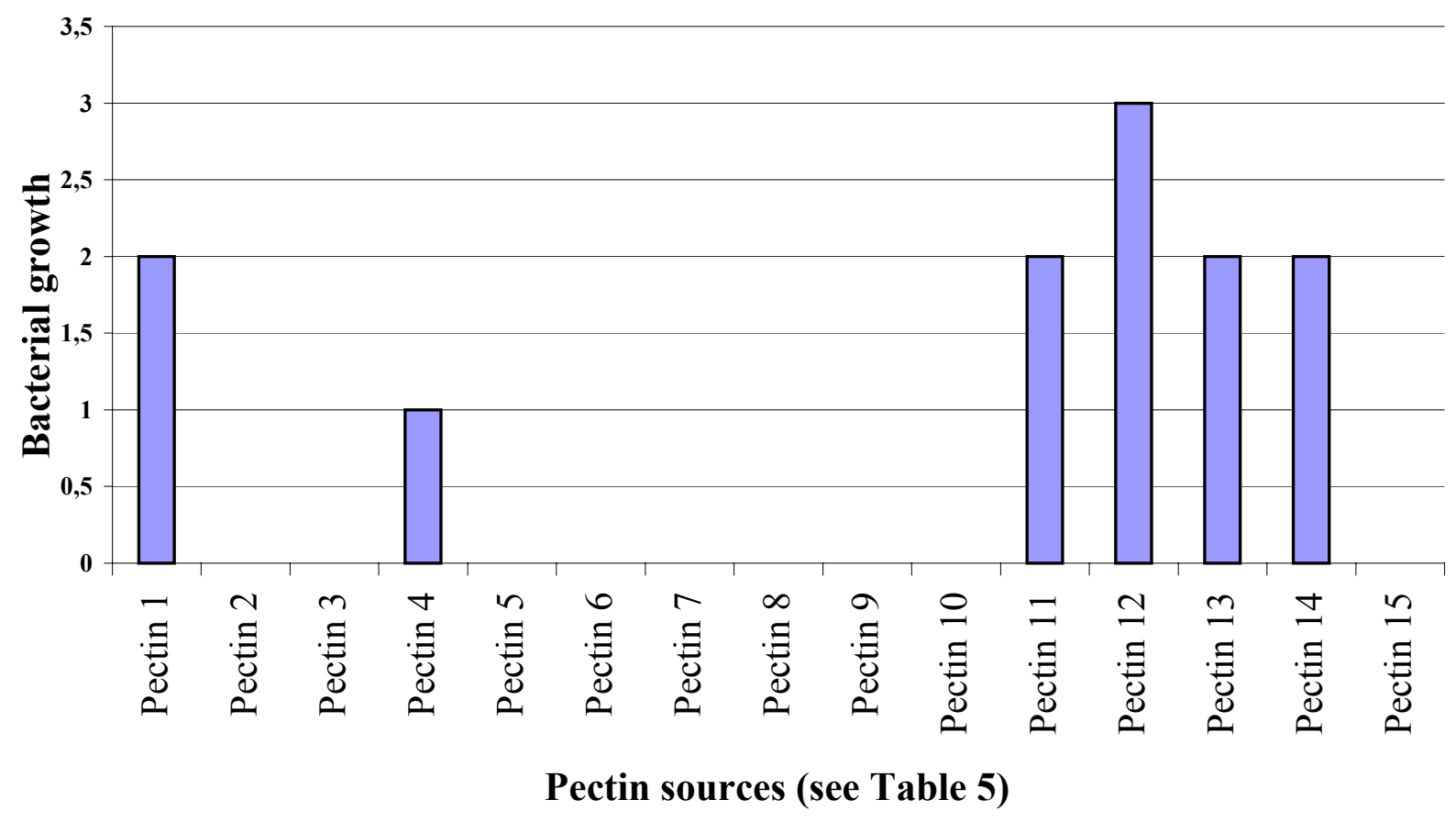

Fig 1: The growth and cavity formation by Erwinia spp. on 15 different pectin sources on the semi-selective medium

\subsubsection{Composition of the new semi-selective medium (CVPM) for isolation soft rot erwinias}

The new semi-selective medium (CVPM) (= crystal violet pectate modified) for isolation of pectolytic Erwinia spp. from potato tubers was developed from the medium of BDLIYA (1995) with the following modifications in the overlayer: reduce the amount of sodiumpolypectate from $18 \mathrm{~g}$ to $15 \mathrm{~g}$ per litre, omit tryptone and bromothymol blue, reduce the amount of $\mathrm{CaCl}_{2} \times 2 \mathrm{H}_{2} \mathrm{O}(10 \%)$ from 6.8 to $6.5 \mathrm{ml}$, and exchange $\mathrm{KNO}_{3}$ with $\mathrm{NaNO}_{3}$ (PEROMBELON and BURNETT, 1991). With the original amount of $18 \mathrm{~g}$ polypectate in the overlayer the medium became too solid and only weak cavities were obtained, omitting of bromothymol blue resulted in more distinctive cavities because the medium of Bdliya (1995) with two indicators (bromothymol blue and crystal violet) became green, so that the appearance of the cavities was not clear. The reduction of the amount of $\mathrm{CaCl}_{2} \times 2 \mathrm{H}_{2} \mathrm{O}(10 \%)$ in the overlayer slightly reduced the firmness of this medium and improved the growth and cavity formation of Erwinia spp., whereas without $\mathrm{CaCl}_{2} \mathrm{X} 2 \mathrm{H}_{2} \mathrm{O}(10 \%)$ the medium did not solidify. 


\section{Basal layer}

Peptone from casein (Roth Co. no.8986.2)

$2.0 \mathrm{~g}$

Yeast extract (Difco no.0127-01)

$0.6 \mathrm{~g}$

$\mathrm{NaCl}$

$1.0 \mathrm{~g}$

SDS

$0.05 \mathrm{~g}$

L-Asparagine (Merck no.1.00126)

$0.5 \mathrm{~g}$

Tryptone (Difco no. 0123-17-3 )

$1.5 \mathrm{~g}$

Agar

$3.0 \mathrm{~g}$

Dist.water

$250 \mathrm{ml}$

pH 7.2

Add $0.5 \mathrm{ml}$ of filter sterilised $1 \%$ solution of 2,3,5-triphenyltetrazolium chloride to the basal layer after autoclaving and cooling down to $50{ }^{\circ} \mathrm{C}$, and add $400 \mu \mathrm{l}(3.2 \mu \mathrm{g} / \mathrm{ml})$ Polymxin B sulphate. Dispense $12 \mathrm{ml}$ of basal medium per plate and allow to set for $10 \mathrm{~min}$.

\section{$\underline{\text { Overlayer }}$}

Crystal violet solution (Sigma no. c-3886)

$1.0 \mathrm{ml} \mathrm{0.075 \% (w/v)}$

$\mathrm{CaCl}_{2} \times 2 \mathrm{H}_{2} \mathrm{O}$

$6.5 \mathrm{ml}(10 \%$ solution $)$

$\mathrm{NaNO}_{3}$

$1.0 \mathrm{~g}$

tri-Na citrate dihydrate

$2.5 \mathrm{~g}$

Agar

$2.0 \mathrm{~g}$

Na-polypectate (Hercules Slendid $\AA$ type 440)

$7.5 \mathrm{~g}$

Dist.water

$500 \mathrm{ml}$

pH 7.2

Add sodiumpolypectate slowly after all other components are dissolved. Adjust pH to 7.2. After autoclaving and cooling down to $45{ }^{\circ} \mathrm{C}$, add $300 \mu \mathrm{l}(1.2 \mu \mathrm{g} / \mathrm{ml})$ Polymxin B sulphate to the overlayer solution, and pour $15 \mathrm{ml} /$ plate on top of the basal layer.

\subsection{Recovery rate and detection limit of Erwinia spp. from artificially contaminated potato extracts}

The details for this experiment are described in chapters 2.3.5 and 2.3.6. In these experiments, $0.1 \mathrm{ml}$ of a standardizerd bacterial suspension from a pure bacterial culture were mixed with potato peel homogenate. Then, aliquots of the mixture were plated on different media. In order to determine the recovery rate and detection limit of the Erwinia spp., the concentration in the 
original bacterial suspension had to be determined by plating on King's medium B. The results are shown in Table 6.

Several laboratory experiments confirmed that potato tubers from different cultivars, which had been washed by tap water, were nearly free from contamination by erwinias, in contrast to unwashed tubers. It was, therefore, concluded that the bacterial numbers listed in tables 7 and 8 are nearly $100 \%$ caused by the artificially added bacterial contamination.

The results obtained in the first experiment show that the recovery rate of bacteria (Fig. 2) from artificially inoculated tuber sap was $87 \%$ on CVPM, whereas the recovery rate was $49 \%$ on the medium of Bdliya (1995) and only $23 \%$ on the CVPB medium of Perombelon \& Burnett (1991) (Table 7).

The first step in this experiment was to estimate the bacterial concentration in the suspensions adjusted to an $\mathrm{OD}_{660}$ of $0.06=($ Table 6$)$. The bacterial concentration in the suspension of the four different E.c. strains varied between $3.6-6.9 \times 10^{7} \mathrm{cfu} / \mathrm{ml}$. These bacterial suspensions were diluted $1: 10^{4}$, and from each strain $0.1 \mathrm{ml}$ were added to $5 \mathrm{ml}$ potato peel homogenate.

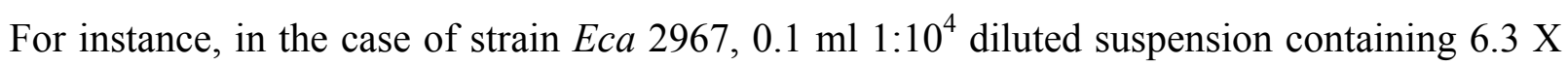
$10^{2}$ cfu were added to $5 \mathrm{ml}$ potato peel homogenate, resulting in a contamination of $1.26 \times 10^{2}$ $\mathrm{cfu} / \mathrm{ml}$ peel homogenate. When $0.1 \mathrm{ml}$ from this mixture were plated on the semi-selective medium, the maximum number of bacterial colonies obtained on the semi-selective medium should be $1.26 \times 10^{1}$, corresponding to $126 \mathrm{cfu}$ per $\mathrm{ml}$ potato peel homogenate. Because only $1.20 \times 10{ }^{1} \mathrm{cfu} / 0.1 \mathrm{ml}$ were recovered on the CVPM medium, the recovery rate (dividing 120 by $126 \mathrm{cfu} / \mathrm{ml}$ ) was $95 \%$ (Table 7 ).

Since the typical cavities for erwinias became only visible on the semi-selective media it was not surprising that more bacterial colonies were counted on King's medium B which does not allow a differentiation of erwinias and non-erwinias. Therefore, the higher numbers of bacterial colonies on King's medium B do not indicate a higher recovery rate of erwinias.

Table 6 : Concentration of bacterial suspensions of O.D. ${ }_{660}=0.06$ determined by dilution plating on King's medium B

\begin{tabular}{|c|c|c|c|c|}
\hline Strain & Bacte & lonis & & $\mathrm{cfu} / \mathrm{ml}$ of original \\
\hline & $1: 10^{4}$ & $1: 10$ & $1: 10^{6}$ & suspension \\
\hline Eca 2967 & uncountable & 63 & 6 & $6.3 \times 10^{7}$ \\
\hline Eca 1401 & uncountable & 54 & 5 & $5.4 \times 10^{7}$ \\
\hline Ecc 436 & uncountable & 69 & 7 & $6.9 \times 10^{7}$ \\
\hline Ecc 1405 & uncountable & 36 & 4 & $3.6 \times 10^{7}$ \\
\hline
\end{tabular}


Table 7: Recovery rate of bacteria from artificially contaminated potato homogenate (bacterial dilution $1: 10^{4}$ ) on four different media. $0.1 \mathrm{ml}$ of each bacterial suspension containing 630, 540,690 and $360 \mathrm{cfu} / \mathrm{ml}$, respectively, were added to $5 \mathrm{ml}$ potato peel homogenate.

\begin{tabular}{|l|c|c|c|c|}
\hline \multirow{2}{*}{ Strain } & \multicolumn{4}{|c|}{ Determined concentration of bacteria (cfu/ml) on } \\
\cline { 2 - 5 } & CVPM & Bdliya 1995 & CVPB & King's B \\
\hline Eca 2967 & $120: 126=95 \%$ & $40: 126=32 \%$ & $40: 126=32 \%$ & $120: 126=95 \%$ \\
\hline Eca 1401 & $90: 108=83 \%$ & $30: 108=28 \%$ & $20: 108=19 \%$ & $100: 108=93 \%$ \\
\hline Ecc 436 & $120: 138=87 \%$ & $70: 138=51 \%$ & $20: 138=14 \%$ & $130: 138=94 \%$ \\
\hline Ecc 1405 & $60: 72=83 \%$ & $60: 72=83 \%$ & $20: 72=28 \%$ & $70: 72=97 \%$ \\
\hline Average & $87 \%$ & $49 \%$ & $23 \%$ & $(95 \%)$ \\
\hline
\end{tabular}

1) Since erwinias could not be differentiated from saprophytic bacteria on King's medium B, the calculated $95 \%$ do not stand for recovery rate of erwinias.

Table 8 : Detection limit of bacteria (bacterial dilution $1: 10^{5}$ ) on four different media. $0.5 \mathrm{ml}$ of the bacterial dilution $1: 10^{5}$ from each bacterial strain containing $315,270,345$ and 180 $\mathrm{cfu} / \mathrm{ml}$, respectively, were added to $5 \mathrm{ml}$ potato peel homogenate.

\begin{tabular}{|l|c|c|c|c|}
\hline \multirow{2}{*}{ Strain } & \multicolumn{4}{|c|}{ Determined concentration of bacteria (cfu/ml potato sap) on } \\
\cline { 2 - 5 } & CVPM & Bdliya 1995 & CVPB & King's B \\
\hline Eca 2967 & $60: 63=95 \%$ & $20: 63=32 \%$ & $0: 63=0 \%$ & $60: 63=95 \%$ \\
\hline Eca 1401 & $40: 54=74 \%$ & $50: 54=93 \%$ & $50: 54=93 \%$ & $60: 54=111 \%$ \\
\hline Ecc 436 & $70: 69=101 \%$ & $50: 69=72 \%$ & $50: 69=72 \%$ & $60: 69=87 \%$ \\
\hline Ecc 1405 & $30: 36=83 \%$ & $20: 36=56 \%$ & $0: 36=0 \%$ & $50: 36=139 \%$ \\
\hline
\end{tabular}

It can be concluded from table 8 that the detection limit on the new semi-selective medium was at least $3.6 \times 10^{1} \mathrm{cfu} / \mathrm{ml}$. Theoretically, the detection limit could be lowered to one $\mathrm{cfu} /$ $0.1 \mathrm{ml}$ extract, i.e. when the bacterial concentration in the original potato homogenate was $1 \times 10^{1} \mathrm{cfu} / \mathrm{ml}$. However, in this case the plating of $0.1 \mathrm{ml}$ from the potato extract on the semiselective medium should be repeated several times. Therefore, it is more realistic to estimate the detection limit with $2 \times \mathbf{1 0}^{\mathbf{1}} \mathbf{c f u} / \mathbf{m l}$. 


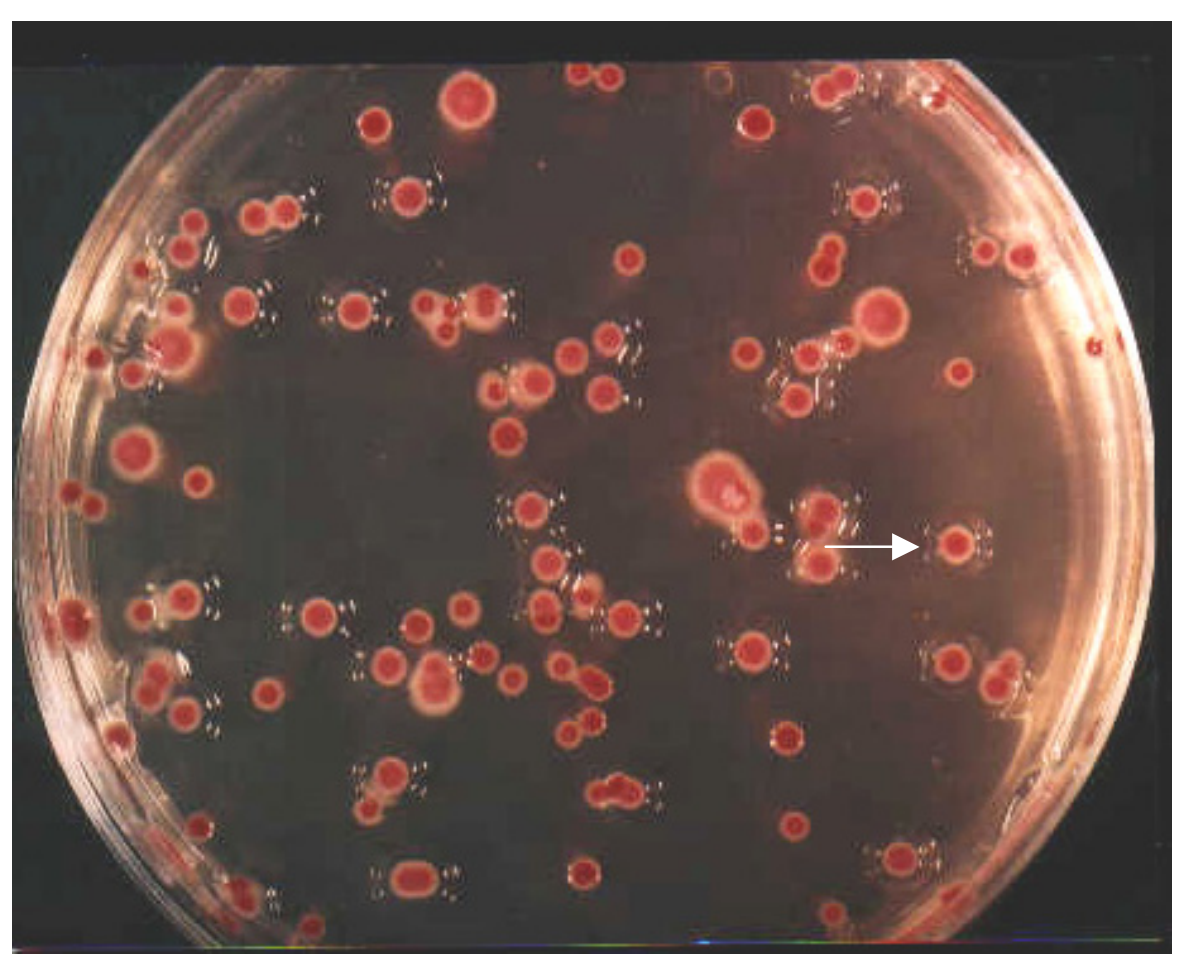

Fig. 2 : Recovery of soft-rot erwinias from artificially inoculated potato peel extract after $24 \mathrm{~h}$

\subsection{Application of the new semi-selective medium for detection of Erwinia spp. from potato tuber peels using two different methods}

The methods for these experiments are described in 2.3.7. The results obtained show that higher numbers of Erwinia spp. colonies were obtained from potato tubers by the soaking method compared to potato tuber peel extracts (Table 9). Obviously, soaking of tubers in 0.01 $\mathrm{M} \mathrm{MgSO}_{4}$ for $3 \mathrm{~h}$ allowed that nearly all Erwinia spp. contaminants diffused from the potato tubers into the soaking solution, because tuber contamination by erwinias is usually on the tuber surface. Soaking for only $3 \mathrm{~h}$ saved time and did not allow the bacteria to multipliy because the temperature was very low $\left(4{ }^{\circ} \mathrm{C}\right)$. In 10 from 11 samples tested lower numbers of erwinias were obtained from peel homogenates than from soakates (Table 9), and in one case with very low bacterial contamination (no.18) bacterial colonies were only obtained from the soakate. Interestingly, all the bacterial strains isolated from the naturally contaminated potato tubers were $E c c$. 
Table 9: Detection and isolation of Ewinia spp. from 12 potato tuber samples using the semiselective medium

\begin{tabular}{|c|c|c|c|}
\hline $\begin{array}{l}\text { Sample } \\
\text { Nr. }\end{array}$ & $\begin{array}{l}\text { Bacterial concentration } \\
\text { cfu/ml homogenate } \\
\text { (potato tuber peels method) }\end{array}$ & $\begin{array}{l}\text { Bacterial conc. } \\
\text { cfu/ml soakage } \\
\text { (soaking method) }\end{array}$ & $\begin{array}{l}\text { Identification of Ewinia spp. } \\
\qquad(12 \text { colonies /sample })\end{array}$ \\
\hline 8 & $7.0 \times 10^{1}$ & $6.0 \times 10^{2}$ & \\
\hline 9 & $1.6 \times 10^{4}$ & $1.1 \times 10^{4}$ & $E c c$ \\
\hline 10 & $1.2 \times 10^{2}$ & $5.4 \times 10^{3}$ & $E c c$ \\
\hline 11 & $1.1 \times 10^{3}$ & $2.7 \times 10^{4}$ & $E c c$ \\
\hline 12 & $1.3 \times 10^{3}$ & $2.5 \times 10^{4}$ & \\
\hline 13 & $1.9 \times 10^{2}$ & $3.9 \times 10^{4}$ & \\
\hline 14 & $7.0 \times 10^{2}$ & $4.3 \times 10^{4}$ & \\
\hline 15 & $2.0 \times 10^{1}$ & $6.3 \times 10^{2}$ & $E c c$ \\
\hline 16 & $2.0 \times 10^{1}$ & $3.3 \times 10^{2}$ & $E c c$ \\
\hline 17 & $8.0 \times 10^{1}$ & $2.4 \times 10^{2}$ & \\
\hline 18 & 0 & $3.0 \times 10^{1}$ & \\
\hline 19 & $9.0 \times 10^{1}$ & $1.9 \times 10^{3}$ & \\
\hline
\end{tabular}

\subsection{Isolation and identification of erwinias from potato tubers}

\subsubsection{Identification of soft rot erwinias (Eca, Ecc and Ech ) by physiological tests}

Table 10 shows the characterization of Erwinia spp. by 10 different tests for grouping pectolytic soft rot erwinias into species and subspecies. When the cavities formed by Ecc and Eca strains on the semi-selective medium were compared, those of Ecc appeared to be broader and deeper than those of Eca. The tests confirmed the taxonomic identification of several Eca, Ecc and Ech strains from the GSPB - bacterial collection. All the strains isolated from potato tubers purchased from different shops and supermarkets in this study (no.1-99, see Table 11) were identified as Ecc. 
Table 10: Identification of Erwinia strains from the GSPB (= Göttinger Sammlung phytopathogener Bakterien) and from seed potato tubers by morphological and biochemical tests

\begin{tabular}{|l|c|c|c|c|c|c|c|c|c|c|}
\hline \multicolumn{1}{|c|}{$\begin{array}{c}\text { Strain } \\
\text { No. }\end{array}$} & \multicolumn{3}{|c|}{$\mathrm{T}\left({ }^{\circ} \mathrm{C}\right)$} & $\begin{array}{c}\propto-\text {-Methyl } \\
\text { d-glucoside }\end{array}$ & $\begin{array}{c}\text { Phos- } \\
\text { phatase }\end{array}$ & $\begin{array}{c}\text { Indole } \\
\text { Formation }\end{array}$ & $\begin{array}{c}\text { Logan's } \\
\text { medium }\end{array}$ & $\begin{array}{c}\text { NaCl } \\
(5 \%)\end{array}$ & $\begin{array}{c}\text { Egg } \\
\text { yolk }\end{array}$ & $\begin{array}{c}\text { Gas } \\
\text { from } \\
\text { glucose }\end{array}$ \\
\hline Eca 1401 & + & - & - & red & - & - & s.r. & + & - & - \\
\hline Eca 2231 & + & - & - & red & - & - & s.r. & + & - & - \\
\hline Ecc 1405 & + & + & $(+)$ & white & - & - & $1 . r$. & + & - & - \\
\hline Ecc 426 & + & + & $(+)$ & white & - & - & $1 . r$. & + & - & - \\
\hline Ech1229 & + & + & + & white & + & + & d.r. & - & + & + \\
\hline Ech1860 & + & + & + & white & + & + & d.r. & - & + & + \\
\hline Ech 2236 & + & + & + & white & + & + & d.r. & - & + & + \\
\hline Eca 2962 & + & - & - & red & - & - & s.r. & + & - & - \\
\hline Strains $1-99$ & + & + & $(+)$ & white & - & - & $1 . r$. & + & - & - \\
\hline
\end{tabular}

s.r. $=$ small, red colony, 1.r. $=$ big, light red colony, and d.r. $=$ dark red colony

$(+)=$ weak growth

\subsubsection{Identification of Erwinia spp. from different potato specimens}

In the years 1997 and 199812 potato specimens were purchased from different supermarkets in Göttingen and tested for the presence of Ewinia carotovora ssp. (for method see 2.2.2). In addition, 4 specimens were obtained from freshly harvested field potatoes: Stöver Co.: cultivars Agria and Morene, University farm Reinhausen: cultivars Agata and Forelle. None of the potato tubers tested showed soft rot symptoms. Therefore, it appears very interesting that contamination by E. carotovora was detected in 10 of the 16 seed potato samples on the CVPM medium according to physiological reactions and the biotest (Tables 10 and 11). Erwinia isolates were identified by their ability to form cavities on the agar due to breakdown of pectate by pectolytic enzymes produced by the bacteria (Fig. 3).

Three different temperatures were used to differentiate between $E c a, E c c$ and $E c h$, because $E c h$ is able to grow and form characteristic cavities at all three temperatures, $E c c$ does so at $27^{\circ} \mathrm{C}$ and $35.5^{\circ} \mathrm{C}$ only, and Eca at $27^{\circ} \mathrm{C}$ only (Fig. 3 ). The bacteria from cavities formed after 13 hours on the CVPM-medium were transferred by toothpick to $\mathrm{KB}$ plates to obtain pure cultures. Further tests confirmed that all the 99 bacterial isolates were Ecc, especially when the growth on $\propto$-methyl-d-glucoside was evaluated (Fig. 4 ). These results indicate that Ecc is the main soft rotting Erwinia occurring on stored potatoes in Germany. Although all the potato 
specimens tested looked healthy a latent contamination with Erwinia carotovora spp. was determined in $2 / 3$ (10 from 16) of the samples (Table 11). The cultivars also showed differences in degree of contamination by Erwinia spp.. Thus, the cultivars Agria and Morene from Stöver Co. were free from the contamination, whereas bacterial numbers from 5-29 per dilution were recorded for several cultivars from different supermarkets. The cultivar Linda from Aldi supermarket was tested at three different times and found to be free from the contamination on March 5 and May 10, whereas after four months (July 1) erwinias were isolated from this cultivar. These results indicated that in the early stage during storage the tubers were relatively free from erwinias, but later on the bacteria could be detected because the conditions during storage were suitable for the growth of the potato soft rot bacteria. Also, on cultivar Hansa from Löb supermarket the degree of bacterial contamination increased from January 15 to April 3, 1998.

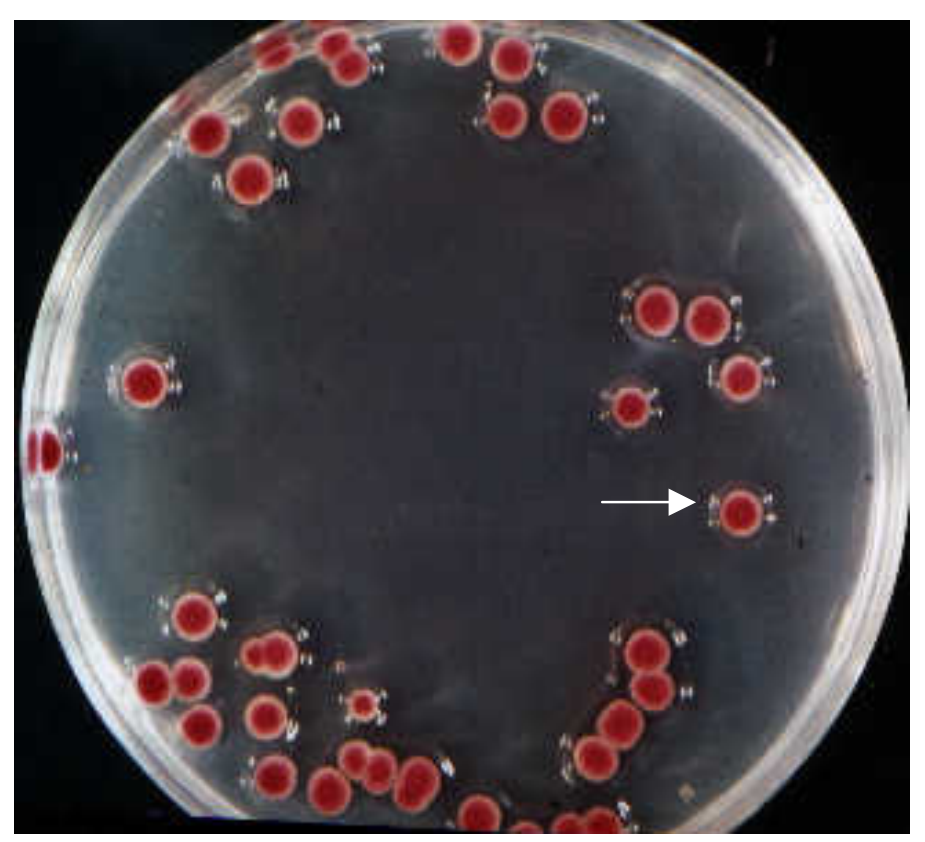

Fig 3: Cavity formation by soft-rot erwinias after incubation at $27^{\circ} \mathrm{C}$ for $24 \mathrm{~h}$ on the CVPM medium. 
Table 11: Source and number of Erwinia strains isolated from different potato tuber cultivars

\begin{tabular}{|l|l|l|l|c|c|c|c|}
\hline Date & Source & Cultivar & $\begin{array}{l}\text { Cavity } \\
\text { colonies }\end{array}$ & $\begin{array}{l}\text { Saproph. } \\
\text { bacteria }\end{array}$ & $\begin{array}{l}\text { Transferred } \\
\text { isolates }\end{array}$ & Biotest & Identification \\
\hline 25.11 .97 & Stöver Co. & Agria & - & many & - & - & - \\
\hline 15.1 .98 & Löb superm. & Hansa & 14 & many & 7 & ++ & $E c c$ \\
\hline 15.2 .98 & Stöver Co. & Morene & - & many & - & - & - \\
\hline 5.3 .98 & Aldi superm. & Linda & - & many & - & - & - \\
\hline 21.3 .98 & Real superm. & Satina & 5 & many & 5 & ++ & $E c c$ \\
\hline 3.4 .98 & Löb superm. & Hansa & 29 & many & 28 & ++ & $E c c$ \\
\hline 15.4 .98 & Penny superm. & Nicola & 3 & many & 3 & ++ & $E c c$ \\
\hline 20.4 .98 & Penny superm. & Spunta & 29 & many & 23 & ++ & $E c c$ \\
\hline 28.4 .98 & Aldi superm. & Renate & 15 & many & 10 & ++ & $E c c$ \\
\hline 10.5 .98 & Aldi superm. & Linda & - & many & - & - & - \\
\hline 12.5 .98 & Löb superm. & Cilena & 5 & many & 5 & ++ & $E c c$ \\
\hline 15.5 .98 & Löb superm. & Diamant & - & many & - & - & - \\
\hline 1.7 .98 & Aldi superm. & Linda & 15 & many & 10 & ++ & $E c c$ \\
\hline 10.8 .98 & $\begin{array}{l}\text { Field of } \\
\text { university farm }\end{array}$ & $\begin{array}{l}\text { Agata } \\
\text { Forelle }\end{array}$ & - & $\begin{array}{c}\text { many } \\
\text { many }\end{array}$ & 4 & ++ & $E c c$ \\
\hline 10.8 .98 & Real superm. & Bebera & 5 & many & 4 & ++ & $E c c$ \\
\hline
\end{tabular}

(A)

(B)

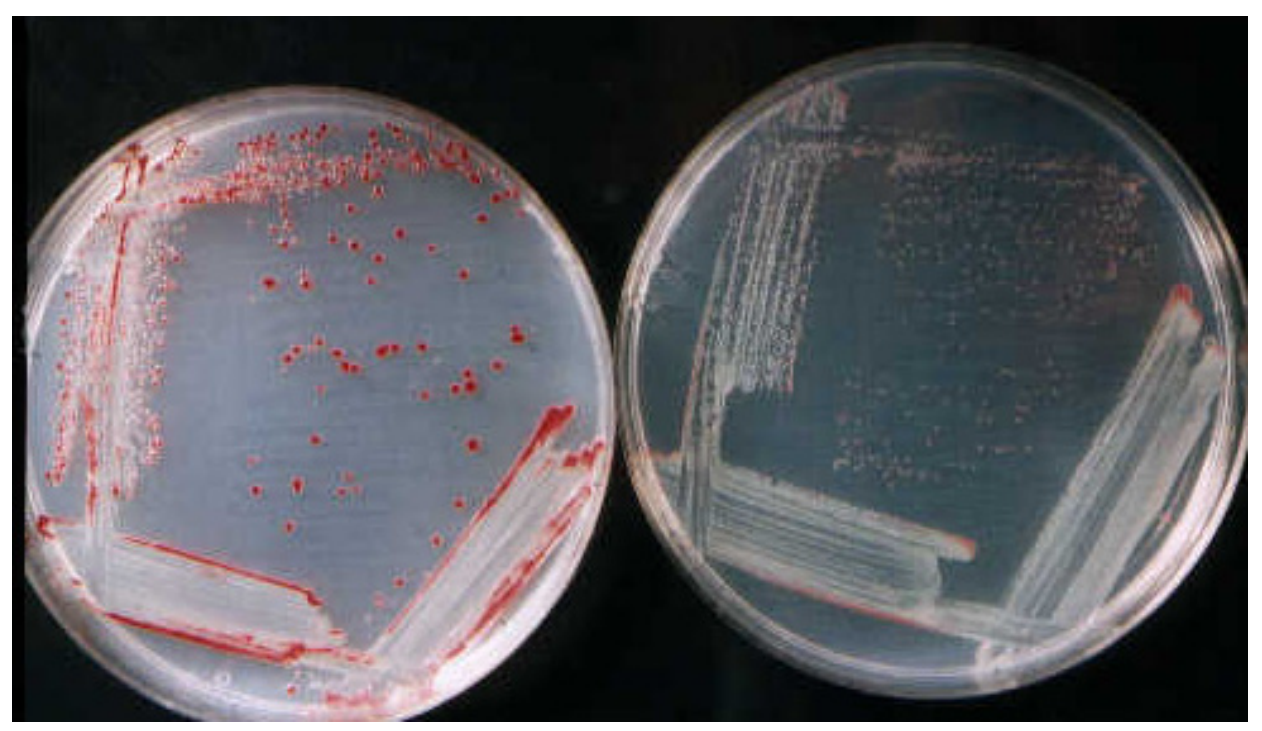

Fig 4: Differentiation between Erwinia carotovora subsp. atroseptica (A) and Erwinia carotovora subsp. carotovora (B) using the $\propto$-methyl-d-glucoside medium after incubation at $27^{\circ} \mathrm{C}$ for $24 \mathrm{~h}$. 


\subsection{Detection of pectolytic Erwinia spp. on single potato tubers and in groups of potato tubers}

It was the aim of these studies to find out, whether latent contamination by erwinias is spread more or less evenly in stored potatoes, or whether only very few potato tubers are contaminated. Only after these experiments it was possible to determine the sample size of the potato specimens to be analysed for latent contamination by erwinias.

As shown in table 12, only three potato tubers from 40 tubers tested were contaminated by Erwinia spp.. However, when groups of potato tubers each consisting of 20 tubers were tested, 4 groups from 5 tested were contaminated, but by different quantities of erwinias (Table 13). It was concluded from these experiments that, for quantifying Erwinia spp. contamination of a potato stock, a representative sample size should consist of 20 tubers taking randomly. Depending on the size of the potato stock, this test should be repeated at least three times with 20 potato tubers in each repetition.

Table 12: Isolation of Erwinia spp. from 40 separately soaked potato tubers using the semi-selective medium

\begin{tabular}{|c|c|}
\hline Tuber no. & Bacterial cfu/ml homogenate \\
\hline 2 & $6.9 \times 10^{2}$ \\
\hline 31 & $3.7 \times 10^{3}$ \\
\hline 36 & $6.3 \times 10^{2}$ \\
\hline $\mathrm{X}$ & 0 \\
\hline
\end{tabular}

$\mathrm{X}=37$ potato tubers were free from latent contamination

Table 13: Detection of Erwinia spp. from 5 potato tuber groups each consisting of 20 tubers

\begin{tabular}{|c|c|}
\hline Group no. & Bacterial cfu/ml homogenate \\
\hline 1 & $6.8 \times 10^{4}$ \\
\hline 2 & 0 \\
\hline 3 & $2.2 \times 10^{3}$ \\
\hline 4 & $1.1 \times 10^{4}$ \\
\hline 5 & $6.3 \times 10^{2}$ \\
\hline
\end{tabular}




\subsection{Detection of latent contamination with Erwinia carotovora spp. in} different potato tuber samples from Stoever Produktion GmbH \&

\section{Co. KG}

The newly developed semi-selective medium was tested on its practical suitability in the frame of an extensive cooperation with Stoever Co.. In this cooperation 231 potato samples were tested for latent contamination with Erwinia spp.. The aim of this investigation was to classify the potato samples according to their grade of contamination with Erwinia spp.. On the basis of these studies, heavily contaminated samples should not be planted in the next season in order to avoid high yield losses due to new infections by Erwinia spp..

Because Stoever Co. exclusively supplies all McDonalds restaurants in Germany with potatoes for food processing, fresh potatoes of high quality must be available all around the year. Therefore, we additionally tested a large lot of consumption potato samples on contamination with Erwinia spp.. Heavily contaminated potato stocks were processed first while the stocks free from Erwinia spp. were processed at the end of the season.

\subsubsection{Comparison between laboratory tests and field observations}

On October 25, 1998, 12 samples of potato tubers harvested in Septemper 1998 were sent to Göttingen by Stöver Co. In these healthy looking specimens latent contamination with Erwinia carotovora spp. was determined as described in chapter 2.3.7.2. The results obtained (Table 14) show a considerable variation in bacterial contamination from "very weak" to "very strong". These laboratory tests in Göttingen were compared with independent observations by experts from Stoever Produktion Co., as shown in Table 14. The scoring of the potato samples in the 3 grades (weak, moderate and strong) by Stöver Co. was based on the following criteria: visible appearance of potato tubers, knowledge on susceptibility of different cultivars, optical impression of field plants, and conditions on the field, such as soil type and soil humidity. Thus, the grading by Stöver company was completely independent of any estimation of bacterial attack and only based on the experience of the experts.

A comparison of these two independent assessments of the potato specimens from different sources revealed a rather good correspondence in most of the cases. The only two exceptions were nos. 9 and 16 which were found to be very strongly contaminated by erwinias (laboratory tests in Göttingen), but assessed as moderately affected by Stöver Co. experts (Table 14). 
Table 14: Comparison of the determined latent contamination with Erwinia spp. in 12 potato tuber samples in Göttingen with field data obtained by Stoever Company in 1998.

\begin{tabular}{|l|l|l|}
\hline Sample no. & Laboratory tests, Göttingen & Field observations, Stöver Co. \\
\hline 8 & weak & moderate 1) \\
\hline 9 & very strong & moderate 2) \\
\hline 10 & moderate & moderate \\
\hline 11 & very strong & strong \\
\hline 12 & strong & strong \\
\hline 13 & strong & strong \\
\hline 14 & moderate & moderate \\
\hline 15 & strong & strong \\
\hline 16 & very strong & moderate \\
\hline 17 & moderate & weak \\
\hline 18 & very weak & weak \\
\hline 19 & moderate & moderate \\
\hline
\end{tabular}

1) $=$ potato tuber sample no. 8 was used as a control and was not treated with Degaclean

2) $=$ potato tuber sample no. 9 was treated with Degaclean (see chapter II)

\subsubsection{Evaluation of latent contamination of 232 potato tuber samples from the years 1998, 1999 and 2000}

In the years of 1998, 1999 and 2000232 samples of potato tubers were sent to Göttingen by Stöver Co. for determination of latent contamination. The potato specimens originated from 3 different locations: Carolinensiel (Ca) near Wittmund (Ostfriesland), 18334 Semlow (Se) near Ribnitz-Dammgarten and 23968 Gägelow (Gä.) near Wismar (Mecklemburg).

In each year, the potatoes were planted between April 1 to April 20 and harvested between September 1 to 30 . Twenty potato tubers from each sample were analysed separately in triplicates as described (2.3.7.2) using the CVPM medium, and classified according to the bacterial concentration per Petri dish and also per tuber. Tables 15, 16 and 17 summarize the results obtained in the years 1998, 1999 and 2001, respectively. These results reveal considerable differences between the samples. In general, the specimens from the year of 1998 were much stronger contaminated than those of the years 1999 and 2000. The lowest contamination was determined for the potatoes harvested in 2000. Thus, the average degrees of contamination were 2.13, 1.24 and 0.42 for the years 1998, 1999 and 2000, respectively. 
Table 15 : Detection of latent contamination with Erwinia spp. of potato tubers harvested in the year 1998

\begin{tabular}{|c|c|c|c|c|c|c|c|c|c|c|c|}
\hline \multirow[b]{2}{*}{$\begin{array}{l}\text { Sample } \\
\text { no. }\end{array}$} & \multicolumn{3}{|c|}{ Experiment 1} & \multicolumn{3}{|c|}{ Experiment 2} & \multicolumn{3}{|c|}{ Experiment 3} & \multirow[b]{2}{*}{ 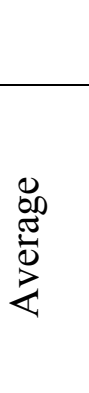 } & \multirow[b]{2}{*}{ 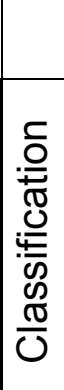 } \\
\hline & 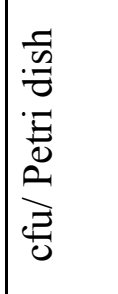 & 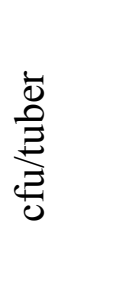 & 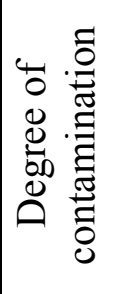 & $\begin{array}{l}\frac{1}{0} \\
: 0 \\
0 \\
\Xi \\
0 \\
0 \\
0 \\
0 \\
0\end{array}$ & $\begin{array}{l}\dot{\bar{\nu}} \\
\stackrel{0}{E} \\
\stackrel{己}{0}\end{array}$ & 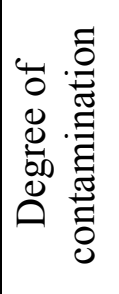 & 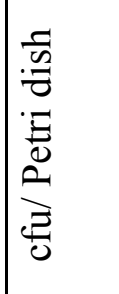 & $\frac{\overline{0}}{\underbrace{\prime}_{0}}$ & 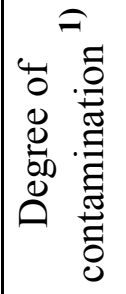 & & \\
\hline Ca.1 & 3 & 45 & 2 & 27 & 405 & 3 & 0 & 0 & 1 & 2.0 & 2 \\
\hline Ca. 2 & 245 & 3675 & 4 & 3 & 45 & 2 & 3 & 45 & 2 & 2.7 & 3 \\
\hline Ca.3 & 1 & 15 & 1 & 3 & 45 & 2 & 0 & 0 & 1 & 1.3 & 1 \\
\hline Ca.4 & 0 & 0 & 1 & 0 & 0 & 1 & 1 & 15 & 1 & 1.0 & 1 \\
\hline Ca.5 & 2 & 30 & 2 & 1 & 15 & 1 & 0 & 0 & 1 & 1.3 & 1 \\
\hline Ca.6 & 6 & 90 & 2 & 1 & 15 & 1 & 0 & 0 & 1 & 1.3 & 1 \\
\hline Ca.7 & 0 & 0 & 1 & 0 & 0 & 1 & 0 & 0 & 1 & 1.0 & 1 \\
\hline Ca. 8 & 37 & 555 & 3 & 0 & 0 & 1 & 1 & 15 & 1 & 1.7 & 2 \\
\hline Ca.9 & 6 & 90 & 2 & 1 & 15 & 1 & 12 & 120 & 2 & 2.0 & 2 \\
\hline Ca.10 & 825 & 12375 & 5 & 3 & 45 & 2 & 7 & 105 & 2 & 3.0 & 3 \\
\hline Ca.11 & 0 & 0 & 1 & 0 & 0 & 1 & 0 & 0 & 1 & 1.0 & 1 \\
\hline Ca.12 & 6 & 90 & 2 & 0 & 0 & 1 & 2 & 30 & 2 & 1.7 & 2 \\
\hline \begin{tabular}{|l|} 
Ca. 13 \\
\end{tabular} & 5 & 75 & 2 & 12 & 180 & 2 & 9 & 90 & 2 & 2.0 & 2 \\
\hline \begin{tabular}{|l|} 
Ca. 14 \\
\end{tabular} & 10 & 150 & 2 & 8 & 120 & 2 & 1 & 15 & 1 & 1.7 & 2 \\
\hline Ca.15 & 25 & 375 & 3 & 1 & 15 & 1 & 0 & 0 & 1 & 1.7 & 2 \\
\hline Ca.16 & 7 & 105 & 2 & 0 & 0 & 1 & 0 & 0 & 1 & 1.3 & 1 \\
\hline \begin{tabular}{|l|} 
Ca. 17 \\
\end{tabular} & 450 & 6750 & 4 & 140 & 2100 & 4 & 490 & 7350 & 4 & 4.0 & 4 \\
\hline Ca.18 & 500 & 7500 & 4 & 0 & 0 & 1 & 2 & 30 & 2 & 2.3 & 2 \\
\hline Ca.19 & 180 & 2700 & 4 & 0 & 0 & 1 & 0 & 0 & 1 & 2.0 & 2 \\
\hline Ca.20 & 10 & 150 & 2 & 0 & 0 & 1 & 0 & 0 & 1 & 1.3 & 1 \\
\hline Ca.21 & 80 & 1200 & 3 & 0 & 0 & 1 & 0 & 0 & 1 & 1.7 & 2 \\
\hline \begin{tabular}{|l|} 
Ca. 22 \\
\end{tabular} & 685 & 10275 & 5 & 1300 & 19500 & 5 & 2 & 30 & 2 & 4.0 & 4 \\
\hline Ca.23 & 370 & 5550 & 4 & 1440 & 21600 & 5 & 45 & 675 & 3 & 4.0 & 4 \\
\hline \begin{tabular}{|l|} 
Ca. 24 \\
\end{tabular} & 80 & 1200 & 3 & 0 & 0 & 1 & 0 & 0 & 1 & 1.7 & 2 \\
\hline Ca.25 & 0 & 0 & 1 & 6 & 90 & 2 & 0 & 0 & 1 & 1.3 & 1 \\
\hline \begin{tabular}{|l|} 
Ca.26 \\
\end{tabular} & 0 & 0 & 1 & 0 & 0 & 1 & 86 & 1290 & 3 & 1.7 & 2 \\
\hline \begin{tabular}{|l} 
Ca.27 \\
\end{tabular} & 45 & 675 & 3 & 34 & 510 & 3 & 26 & 390 & 3 & 3.0 & 3 \\
\hline \begin{tabular}{|l|} 
Ca. 28 \\
\end{tabular} & 0 & 0 & 1 & 5 & 75 & 2 & 1 & 15 & 1 & 1.3 & 1 \\
\hline \begin{tabular}{|l} 
Ca.29 \\
\end{tabular} & 420 & 6300 & 4 & 370 & 5550 & 4 & 0 & 0 & 1 & 3.0 & 3 \\
\hline \begin{tabular}{|l|} 
Ca.30 \\
\end{tabular} & 0 & 0 & 1 & 260 & 3900 & 4 & 450 & 6750 & 4 & 3.0 & 3 \\
\hline \begin{tabular}{|l|} 
Ca.31 \\
\end{tabular} & 320 & 4800 & 4 & 0 & 0 & 1 & 12 & 180 & 2 & 2.3 & 2 \\
\hline \begin{tabular}{|l|} 
Ca.32 \\
\end{tabular} & 3 & 45 & 2 & 160 & 2400 & 4 & 0 & 0 & 1 & 2.3 & 2 \\
\hline \begin{tabular}{|l|} 
Ca.33 \\
\end{tabular} & 400 & 6000 & 4 & 0 & 0 & 1 & 0 & 0 & 1 & 2.0 & 2 \\
\hline \begin{tabular}{|l|} 
Se.1 \\
\end{tabular} & 26 & 390 & 3 & 2 & 30 & 2 & 22 & 330 & 3 & 2.7 & 3 \\
\hline \begin{tabular}{|l|} 
Se. 2 \\
\end{tabular} & 0 & 0 & 1 & 0 & 0 & 1 & 0 & 0 & 1 & 1.0 & 1 \\
\hline Se. 3 & 1 & 15 & 1 & 0 & 0 & 1 & 0 & 0 & 1 & 1.0 & 1 \\
\hline \begin{tabular}{|l|} 
Se.4 \\
\end{tabular} & 35 & 525 & 3 & 1 & 15 & 1 & 41 & 615 & 3 & 2.3 & 2 \\
\hline \begin{tabular}{|l|} 
Se. 5 \\
\end{tabular} & 137 & 2055 & 4 & 2 & 30 & 2 & 4 & 60 & 2 & 2.7 & 3 \\
\hline
\end{tabular}




\begin{tabular}{|c|c|c|c|c|c|c|c|c|c|c|c|}
\hline Se. 6 & 6 & 90 & 2 & 165 & 2475 & 4 & 0 & 0 & 1 & 2.3 & 2 \\
\hline Se.7 & 47 & 705 & 3 & 135 & 2025 & 4 & 0 & 0 & 1 & 2.7 & 3 \\
\hline Se. 8 & 24 & 360 & 3 & 8 & 120 & 2 & 1360 & 20400 & 5 & 3.3 & 3 \\
\hline Se. 9 & 34 & 510 & 3 & 182 & 2730 & 4 & 0 & 0 & 1 & 2.7 & 3 \\
\hline Se.10 & 0 & 0 & 1 & 1 & 15 & 1 & 0 & 0 & 1 & 1.0 & 1 \\
\hline Se.11 & 9 & 135 & 2 & 0 & 0 & 1 & 0 & 0 & 1 & 1.3 & 1 \\
\hline Se.12 & 0 & 0 & 1 & 1 & 15 & 1 & 0 & 0 & 1 & 1.0 & 1 \\
\hline Se.13 & 6 & 90 & 2 & 0 & 0 & 1 & 0 & 0 & 1 & 1.3 & 1 \\
\hline Se.14 & 60 & 900 & 3 & 0 & 0 & 1 & 465 & 6975 & 4 & 2.7 & 3 \\
\hline Se.15 & 9 & 135 & 2 & 0 & 0 & 1 & 4000 & 60000 & 5 & 2.7 & 3 \\
\hline Se.16 & 0 & 0 & 1 & 0 & 0 & 1 & 0 & 0 & 1 & 1.0 & 1 \\
\hline Se. 17 & 10 & 150 & 2 & 0 & 0 & 1 & 0 & 0 & 1 & 1.3 & 1 \\
\hline Gä.1 & 127 & 1905 & 3 & 15 & 225 & 3 & 140 & 2100 & 4 & 3.3 & 3 \\
\hline Gä.2 & 16 & 240 & 3 & 121 & 1815 & 3 & 11 & 165 & 2 & 2.7 & 3 \\
\hline Gä.3 & 4 & 60 & 2 & 0 & 0 & 1 & 0 & 0 & 1 & 1.3 & 1 \\
\hline Gä.4 & 4 & 60 & 2 & 0 & 0 & 1 & 0 & 0 & 1 & 1.3 & 1 \\
\hline Gä.5 & 200 & 3000 & 4 & 6 & 90 & 2 & 28 & 420 & 3 & 3.0 & 3 \\
\hline Gä.6 & 38 & 570 & 3 & 0 & 0 & 1 & 44 & 660 & 3 & 2.3 & 2 \\
\hline Gä.7 & 13 & 195 & 2 & 6 & 90 & 2 & 35 & 525 & 3 & 2.3 & 2 \\
\hline Gä.8 & 41 & 615 & 3 & 5 & 75 & 2 & 125 & 1875 & 3 & 2.7 & 3 \\
\hline Gä.9 & 1 & 15 & 1 & 0 & 0 & 1 & 3 & 45 & 2 & 1.3 & 1 \\
\hline Gä.10 & 19 & 285 & 3 & 0 & 0 & 1 & 15 & 225 & 3 & 2.3 & 2 \\
\hline Gä.11 & 118 & 1770 & 3 & 5 & 75 & 2 & 130 & 1950 & 3 & 2.7 & 3 \\
\hline Gä.12 & 42 & 630 & 3 & 20 & 300 & 3 & 5 & 75 & 2 & 2.7 & 3 \\
\hline Gä.13 & 27 & 405 & 3 & 77 & 1155 & 3 & 92 & 1380 & 3 & 2.7 & 3 \\
\hline Gä.14 & 53 & 795 & 3 & 98 & 1470 & 3 & 69 & 1035 & 3 & 2.7 & 3 \\
\hline Gä.15 & 357 & 5355 & 4 & 265 & 3975 & 4 & 90 & 1350 & 3 & 3.3 & 3 \\
\hline Gä.16 & 262 & 3930 & 4 & 127 & 1905 & 3 & 31 & 465 & 3 & 3.0 & 3 \\
\hline BA.1 & 0 & 0 & 1 & 20 & 300 & 3 & 6 & 90 & 2 & 2.0 & 2 \\
\hline BA.2 & 6 & 90 & 2 & 0 & 0 & 1 & 0 & 0 & 1 & 1.3 & 1 \\
\hline BA.3 & 6 & 90 & 2 & 1 & 15 & 1 & 0 & 0 & 1 & 1.3 & 1 \\
\hline BA.4 & 30 & 450 & 3 & 0 & 0 & 1 & 0 & 0 & 1 & 1.7 & 2 \\
\hline BA. 5 & 30 & 450 & 3 & 149 & 2235 & 4 & 17 & 255 & 3 & 3.3 & 3 \\
\hline BA.6 & 30 & 450 & 3 & 2 & 30 & 2 & 0 & 0 & 1 & 2.0 & 2 \\
\hline BA.7 & 70 & 1050 & 3 & 4 & 60 & 2 & 2 & 60 & 2 & 2.3 & 2 \\
\hline BA.8 & 240 & 3600 & 4 & 30 & 450 & 3 & 34 & 1020 & 3 & 3.3 & 3 \\
\hline \multicolumn{10}{|c|}{ Average degree of contamination } & 2.13 & \\
\hline
\end{tabular}

1) Footnote for table 15 :

Degree of contamination

\begin{tabular}{|c|c|}
\hline 0 & Free of contamination \\
\hline 1 & very weak \\
\hline 2 & weak \\
\hline 3 & moderate \\
\hline 4 & strong \\
\hline 5 & very strong \\
\hline
\end{tabular}


Table 16 : Detection of latent contamination with Erwinia spp. of potato tubers harvested in the year 1999

\begin{tabular}{|c|c|c|c|c|c|c|c|c|}
\hline \multirow[b]{2}{*}{ Sample no. } & \multicolumn{3}{|c|}{ Experiment 1} & \multicolumn{3}{|c|}{ Experiment 2} & \multirow[b]{2}{*}{ 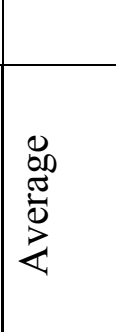 } & \multirow[b]{2}{*}{ 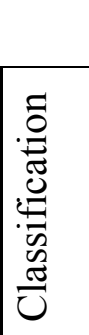 } \\
\hline & $\begin{array}{l}\frac{1}{0} \\
: 0 \\
0 \\
\vdots \\
0 \\
0 \\
己 \\
0 \\
0\end{array}$ & $\begin{array}{l}\dot{\bar{d}} \\
\stackrel{D}{E} \\
\sum_{0}^{0}\end{array}$ & 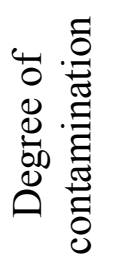 & $\begin{array}{l}\frac{1}{n} \\
: 0 \\
\vdots \\
0 \\
0 \\
0 \\
0 \\
0\end{array}$ & 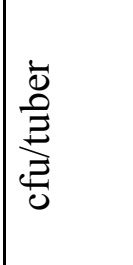 & 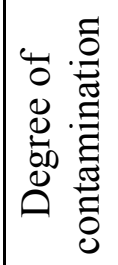 & & \\
\hline Ca.1 & 0 & 0 & 1 & 0 & 0 & 1 & 1.0 & 1 \\
\hline Ca. 2 & 2700 & 40500 & 5 & 3 & 45 & 2 & 3.5 & 4 \\
\hline Ca.3 & 0 & 0 & 1 & 0 & 0 & 1 & 1.0 & 1 \\
\hline Ca.4 & 1 & 15 & 1 & 0 & 0 & 1 & 1.0 & 1 \\
\hline Ca.5 & 0 & 0 & 1 & 0 & 0 & 1 & 1.0 & 1 \\
\hline Ca.6 & 0 & 0 & 1 & 0 & 0 & 1 & 1.0 & 1 \\
\hline Ca.7 & 0 & 0 & 1 & 0 & 0 & 1 & 1.0 & 1 \\
\hline Ca. 8 & 0 & 0 & 1 & 0 & 0 & 1 & 1.0 & 1 \\
\hline Ca.9 & 3 & 45 & 1 & 1 & 15 & 1 & 1.0 & 1 \\
\hline \begin{tabular}{|l|} 
Ca. 10 \\
\end{tabular} & 0 & 0 & 1 & 0 & 0 & 1 & 1.0 & 1 \\
\hline Ca.11 & 12 & 180 & 2 & 0 & 0 & 1 & 1.5 & 2 \\
\hline Ca.12 & 0 & 0 & 1 & 0 & 0 & 1 & 1.0 & 1 \\
\hline Ca.13 & 0 & 0 & 1 & 0 & 0 & 1 & 1.0 & 1 \\
\hline Ca.14 & 2400 & 3600 & 5 & 425 & 6375 & 4 & 4.5 & 5 \\
\hline \begin{tabular}{|l} 
Ca.15 \\
\end{tabular} & 0 & 0 & 1 & 0 & 1 & 1 & 1.0 & 1 \\
\hline Ca.16 & 0 & 0 & 1 & 0 & 1 & 1 & 1.0 & 1 \\
\hline \begin{tabular}{|l|} 
Ca. 17 \\
\end{tabular} & 2 & 30 & 1 & 0 & 1 & 1 & 1.0 & 1 \\
\hline Ca.18 & 0 & 0 & 1 & 0 & 1 & 1 & 1.0 & 1 \\
\hline Ca.19 & 0 & 0 & 1 & 0 & 1 & 1 & 1.0 & 1 \\
\hline Ca.20 & 13 & 195 & 2 & 2 & 30 & 2 & 2.0 & 2 \\
\hline \begin{tabular}{|l|} 
Ca.21 \\
\end{tabular} & 0 & 0 & 1 & 2 & 30 & 2 & 1.5 & 2 \\
\hline \begin{tabular}{|l} 
Ca.22 \\
\end{tabular} & 0 & 0 & 1 & 0 & 0 & 1 & 1.0 & 1 \\
\hline Ca.23 & 0 & 0 & 1 & 0 & 0 & 1 & 1.0 & 1 \\
\hline \begin{tabular}{|l|} 
Ca. 24 \\
\end{tabular} & 0 & 0 & 1 & 0 & 0 & 1 & 1.0 & 1 \\
\hline \begin{tabular}{|l|} 
Ca. 25 \\
\end{tabular} & 0 & 0 & 1 & 0 & 0 & 1 & 1.0 & 1 \\
\hline \begin{tabular}{|l|} 
Ca.26 \\
\end{tabular} & 0 & 0 & 1 & 0 & 0 & 1 & 1.0 & 1 \\
\hline Ca.27 & 24 & 360 & 3 & 0 & 0 & 1 & 2.0 & 2 \\
\hline \begin{tabular}{|l|} 
Ca. 28 \\
\end{tabular} & 0 & 0 & 1 & 0 & 0 & 1 & 1.0 & 1 \\
\hline \begin{tabular}{|l} 
Ca.29 \\
\end{tabular} & 0 & 0 & 1 & 0 & 0 & 1 & 1.0 & 1 \\
\hline Ca.30 & 0 & 0 & 1 & 0 & 0 & 1 & 1.0 & 1 \\
\hline \begin{tabular}{|l|} 
Ca.31 \\
\end{tabular} & 0 & 0 & 1 & 0 & 0 & 1 & 1.0 & 1 \\
\hline Ca.32 & 0 & 0 & 1 & 0 & 0 & 1 & 1.0 & 1 \\
\hline \begin{tabular}{|l|} 
Ca.33 \\
\end{tabular} & 0 & 0 & 3 & 0 & 0 & 1 & 2.0 & 2 \\
\hline \begin{tabular}{|c|} 
Ca.34 \\
\end{tabular} & 26 & 390 & 3 & 0 & 0 & 1 & 2.0 & 2 \\
\hline \begin{tabular}{|l|} 
Ca.35 \\
\end{tabular} & 0 & 0 & 1 & 0 & 0 & 1 & 1.0 & 1 \\
\hline Ca.36 & 4 & 60 & 2 & 0 & 0 & 1 & 1.0 & 1 \\
\hline Ca.37 & 0 & 0 & 1 & 0 & 0 & 1 & 1.0 & 1 \\
\hline Ca.38 & 0 & 0 & 1 & 0 & 0 & 1 & 1.0 & 1 \\
\hline \begin{tabular}{|l|} 
Ca.39 \\
\end{tabular} & 0 & 0 & 1 & 0 & 0 & 1 & 1.0 & 1 \\
\hline $\mathrm{Ca} .40$ & 0 & 0 & 1 & 0 & 0 & 1 & 1.0 & 1 \\
\hline $\mathrm{Ca} .41$ & 0 & 0 & 1 & 0 & 0 & 1 & 1.0 & 1 \\
\hline
\end{tabular}




\begin{tabular}{|c|c|c|c|c|c|c|c|c|}
\hline Ca.42 & 0 & 0 & 1 & 0 & 0 & 1 & 1.0 & 1 \\
\hline Ca.43 & 0 & 0 & 1 & 0 & 0 & 1 & 1.0 & 1 \\
\hline Gä.1 & 0 & 0 & 1 & 0 & 0 & 1 & 1.0 & 1 \\
\hline Gä.2 & 1 & 15 & 1 & 0 & 0 & 1 & 1.0 & 1 \\
\hline Gä.3 & 0 & 0 & 1 & 0 & 0 & 1 & 1.0 & 1 \\
\hline Gä.4 & 0 & 0 & 1 & 0 & 0 & 1 & 1.0 & 1 \\
\hline Gä.5 & 0 & 0 & 1 & 0 & 0 & 1 & 1.0 & 1 \\
\hline Gä.6 & 16 & 240 & 2 & 5 & 75 & 2 & 2.0 & 2 \\
\hline Gä.7 & 1 & 15 & 1 & 0 & 0 & 1 & 1.0 & 1 \\
\hline Gä.8 & 75 & 405 & 3 & 0 & 0 & 1 & 2.0 & 2 \\
\hline Gä.9 & 1 & 15 & 1 & 0 & 0 & 1 & 1.0 & 1 \\
\hline Gä.10 & 0 & 0 & 1 & 0 & 0 & 1 & 1.0 & 1 \\
\hline Gä.11 & 0 & 0 & 1 & 0 & 0 & 1 & 1.0 & 1 \\
\hline \begin{tabular}{|l|} 
Se. 1 \\
\end{tabular} & 0 & 0 & 1 & 0 & 0 & 1 & 1.0 & 1 \\
\hline Se. 2 & 0 & 0 & 1 & 0 & 0 & 1 & 1.0 & 1 \\
\hline Se. 3 & 0 & 0 & 1 & 0 & 0 & 1 & 1.0 & 1 \\
\hline Se.4 & 0 & 0 & 1 & 0 & 0 & 1 & 1.0 & 1 \\
\hline Se. 5 & 0 & 0 & 1 & 0 & 0 & 1 & 1.0 & 1 \\
\hline Se.6 & 0 & 1 & 0 & 0 & 1 & 1 & 1.0 & 1 \\
\hline Se.7 & 1500 & 325000 & 5 & 0 & 0 & 1 & 3.0 & 3 \\
\hline Se. 8 & 0 & 0 & 1 & 0 & 0 & 1 & 1.0 & 1 \\
\hline Se.9 & 0 & 0 & 1 & 0 & 0 & 1 & 1.0 & 1 \\
\hline Se.10 & 0 & 0 & 1 & 0 & 0 & 1 & 1.0 & 1 \\
\hline Se.11 & 8 & 120 & 2 & 0 & 0 & 1 & 1.5 & 2 \\
\hline Se.12 & 0 & 0 & 1 & 0 & 0 & 1 & 1.0 & 2 \\
\hline Se.13 & 3 & 45 & 2 & 0 & 0 & 1 & 1.5 & 2 \\
\hline Se.14 & 0 & 0 & 1 & 0 & 0 & 1 & 1.0 & 1 \\
\hline Se.15 & 28 & 420 & 3 & 0 & 0 & 1 & 2.0 & 2 \\
\hline Se.16 & 0 & 0 & 1 & 0 & 0 & 1 & 1.0 & 1 \\
\hline \begin{tabular}{|l|} 
Se. 17 \\
\end{tabular} & 0 & 0 & 1 & 0 & 0 & 1 & 1.0 & 1 \\
\hline Se.18 & 27 & 405 & 3 & 8 & 120 & 2 & 2.5 & 3 \\
\hline \begin{tabular}{|l|} 
Se.19 \\
\end{tabular} & 0 & 0 & 1 & 0 & 0 & 1 & 1.0 & 1 \\
\hline Se.20 & 0 & 0 & 1 & 0 & 0 & 1 & 1.0 & 1 \\
\hline Bradby 1 & 0 & 0 & 1 & 0 & 0 & 1 & 1.0 & 1 \\
\hline Bradby 2 & 0 & 0 & 1 & 0 & 0 & 1 & 1.0 & 1 \\
\hline \multicolumn{7}{|c|}{ Average degree of contamination } & 1.24 & \\
\hline
\end{tabular}


Table 17 : Detection of latent contamination with Erwinia spp. of potato tubers harvested in the year 2000

\begin{tabular}{|c|c|c|c|c|c|c|c|c|c|c|c|}
\hline & \multicolumn{3}{|c|}{ Experiment 1} & \multicolumn{3}{|c|}{ Experiment 2} & \multicolumn{3}{|c|}{ Experiment 3} & \multirow[b]{2}{*}{ 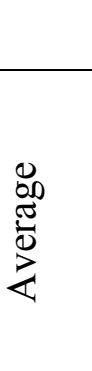 } & \multirow[b]{2}{*}{ 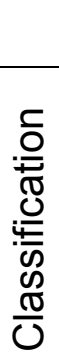 } \\
\hline $\begin{array}{c}\text { Sample } \\
\text { no. }\end{array}$ & 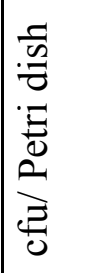 & $\begin{array}{l}\dot{\bar{D}} \\
\stackrel{D}{E} \\
\stackrel{己}{0}\end{array}$ & 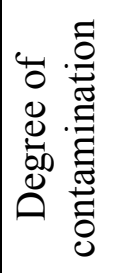 & 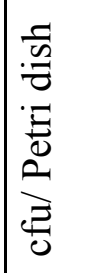 & $\frac{\bar{\Xi}}{\sum_{0}^{D}}$ & 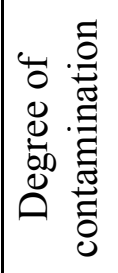 & $\begin{array}{l}\frac{1}{5} \\
: 0 \\
0 \\
\vdots \\
0 \\
2 \\
己 \\
0 \\
0\end{array}$ & $\frac{\bar{D}}{\stackrel{D}{E}}$ & 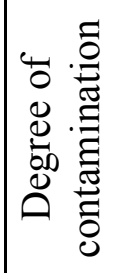 & & \\
\hline Ca.1 & 0 & 0 & 0 & 0 & 0 & 0 & 0 & 0 & 0 & 0 & 0 \\
\hline Ca.2 & 0 & 0 & 0 & 0 & 0 & 0 & 0 & 0 & 0 & 0 & 0 \\
\hline Ca.3 & 0 & 0 & 0 & 0 & 0 & 0 & 0 & 0 & 0 & 0 & 0 \\
\hline Ca.4 & 0 & 0 & 0 & 0 & 0 & 0 & 0 & 0 & 0 & 0 & 0 \\
\hline Ca.5 & 14 & 420 & 3 & 0 & 0 & 0 & 0 & 0 & 0 & 1.0 & 1 \\
\hline Ca.6 & 2 & 60 & 2 & 3 & 90 & 2 & 2 & 60 & 2 & 2.0 & 2 \\
\hline Ca.7 & 0 & 0 & 0 & 0 & 0 & 0 & 0 & 0 & 0 & 0 & 0 \\
\hline Ca. 8 & 0 & 0 & 0 & 0 & 0 & 0 & 0 & 0 & 0 & 0 & 0 \\
\hline Ca.9 & 0 & 0 & 0 & 1 & 30 & 2 & 0 & 0 & 0 & 0.66 & 1 \\
\hline Ca.10 & 0 & 0 & 0 & 0 & 0 & 0 & 0 & 0 & 0 & 0 & 0 \\
\hline Ca.11 & 0 & 0 & 0 & 0 & 0 & 0 & 0 & 0 & 0 & 0 & 0 \\
\hline Ca.12 & 0 & 0 & 0 & 0 & 0 & 0 & 0 & 0 & 0 & 0 & 0 \\
\hline Ca.13 & 0 & 0 & 0 & 0 & 0 & 0 & 0 & 0 & 0 & 0 & 0 \\
\hline Ca.14 & 0 & 0 & 0 & 0 & 0 & 0 & 0 & 0 & 0 & 0 & 0 \\
\hline Ca.15 & 0 & 0 & 0 & 0 & 0 & 0 & 0 & 0 & 0 & 0 & 0 \\
\hline Ca.16 & 0 & 0 & 0 & 0 & 0 & 0 & 0 & 0 & 0 & 0 & 0 \\
\hline Ca.17 & 0 & 0 & 0 & 0 & 0 & 0 & 0 & 0 & 0 & 0 & 0 \\
\hline Ca.18 & 0 & 0 & 0 & 0 & 0 & 0 & 0 & 0 & 0 & 0 & 0 \\
\hline Ca.19 & 0 & 0 & 0 & 0 & 0 & 0 & 0 & 0 & 0 & 0 & 0 \\
\hline Ca.20 & 0 & 0 & 0 & 0 & 0 & 0 & 0 & 0 & 0 & 0 & 0 \\
\hline Ca.21 & 0 & 0 & 0 & 0 & 0 & 0 & 0 & 0 & 0 & 0 & 0 \\
\hline Ca.22 & 0 & 0 & 0 & 0 & 0 & 0 & 0 & 0 & 0 & 0 & 0 \\
\hline Ca.23 & 0 & 0 & 0 & 0 & 0 & 0 & 0 & 0 & 0 & 0 & 0 \\
\hline Ca.24 & 0 & 0 & 0 & 0 & 0 & 0 & 0 & 0 & 0 & 0 & 0 \\
\hline Ca.25 & 0 & 0 & 0 & 0 & 0 & 0 & 1 & 30 & 2 & 0.66 & 1 \\
\hline Ca.26 & 0 & 0 & 0 & 0 & 0 & 0 & 0 & 0 & 0 & 0 & 0 \\
\hline Ca.27 & 1 & 30 & 2 & 5 & 150 & 2 & 0 & 0 & 0 & 1.3 & 1 \\
\hline Ca.28 & 0 & 0 & 0 & 0 & 0 & 0 & 0 & 0 & 0 & 0 & 0 \\
\hline Ca.29 & 0 & 0 & 0 & 0 & 0 & 0 & 0 & 0 & 0 & 0 & 0 \\
\hline Ca.30 & 0 & 0 & 0 & 1 & 30 & 2 & 1 & 30 & 2 & 1.3 & 1 \\
\hline Ca.31 & 0 & 0 & 0 & 0 & 0 & 0 & 0 & 0 & 0 & 0 & 0 \\
\hline Ca.32 & 0 & 0 & 0 & 0 & 0 & 0 & 0 & 0 & 0 & 0 & 0 \\
\hline Ca.33 & 0 & 0 & 0 & 3 & 90 & 2 & 2 & 60 & 2 & 1.3 & 1 \\
\hline Ca.34 & 0 & 0 & 0 & 1 & 30 & 2 & 1 & 30 & 2 & 1.3 & 1 \\
\hline Ca.35 & 0 & 0 & 0 & 0 & 0 & 0 & 0 & 0 & 0 & 0 & 0 \\
\hline Ca.36 & 0 & 0 & 0 & 0 & 0 & 0 & 0 & 0 & 0 & 0 & 0 \\
\hline \begin{tabular}{|l|} 
Ca.37 \\
\end{tabular} & 0 & 0 & 0 & 0 & 0 & 0 & 0 & 0 & 0 & 0 & 0 \\
\hline Ca.38 & 0 & 0 & 0 & 0 & 0 & 0 & 0 & 0 & 0 & 0 & 0 \\
\hline Ca.39 & 0 & 0 & 0 & 0 & 0 & 0 & 0 & 0 & 0 & 0 & 0 \\
\hline
\end{tabular}




\begin{tabular}{|c|c|c|c|c|c|c|c|c|c|c|c|}
\hline Ca.40 & 0 & 0 & 0 & 0 & 0 & 0 & 0 & 0 & 0 & 0 & 0 \\
\hline Ca.41 & 70 & 2100 & 4 & 30 & 900 & 3 & 1 & 30 & 2 & 2.6 & 3 \\
\hline Ca.42 & 0 & 0 & 0 & 0 & 0 & 0 & 0 & 0 & 0 & 0 & 0 \\
\hline $\mathrm{Ca} .43$ & 0 & 0 & 0 & 0 & 0 & 0 & 0 & 0 & 0 & 0 & 0 \\
\hline Ca.44 & 0 & 0 & 0 & 0 & 0 & 0 & 0 & 0 & 0 & 0 & 0 \\
\hline Ca.45 & 0 & 0 & 0 & 0 & 0 & 0 & 0 & 0 & 0 & 0 & 0 \\
\hline Ca.46 & 0 & 0 & 0 & 0 & 0 & 0 & 0 & 0 & 0 & 0 & 0 \\
\hline Gä.1 & 1 & 30 & 2 & 0 & 0 & 0 & 0 & 0 & 0 & 0.67 & 1 \\
\hline Gä.2 & 0 & 0 & 0 & 0 & 0 & 0 & 0 & 0 & 0 & 0 & 0 \\
\hline Gä.3 & 0 & 0 & 0 & 0 & 0 & 0 & 0 & 0 & 0 & 0 & 0 \\
\hline Gä.4 & 13 & 390 & 3 & 23 & 690 & 3 & 23 & 690 & 3 & 3.0 & 3 \\
\hline Gä.5 & 1 & 30 & 2 & 3 & 90 & 2 & 1 & 30 & 2 & 2.0 & 2 \\
\hline Gä.6 & 6 & 180 & 2 & 16 & 480 & 3 & 390 & 1170 & 3 & 2.7 & 3 \\
\hline Gä.7 & 0 & 0 & 0 & 0 & 0 & 0 & 0 & 0 & 0 & 0 & 0 \\
\hline Gä.8 & 1 & 30 & 2 & 1 & 30 & 2 & 1 & 30 & 2 & 1.0 & 1 \\
\hline Gä.9 & 0 & 0 & 0 & 0 & 0 & 0 & 0 & 0 & 0 & 0 & 0 \\
\hline Gä.10 & 0 & 0 & 0 & 0 & 0 & 0 & 0 & 0 & 0 & 0 & 0 \\
\hline Gä.11 & 0 & 0 & 0 & 0 & 0 & 0 & 0 & 0 & 0 & 0 & 0 \\
\hline Se.1 & 0 & 0 & 0 & 0 & 0 & 0 & 0 & 0 & 0 & 0 & 0 \\
\hline Se. 2 & 0 & 0 & 0 & 0 & 0 & 0 & 0 & 0 & 0 & 0 & 0 \\
\hline Se. 3 & 0 & 0 & 0 & 0 & 0 & 0 & 0 & 0 & 0 & 0 & 0 \\
\hline Se. 4 & 3 & 90 & 2 & 3 & 90 & 2 & 1 & 30 & 2 & 2.0 & 2 \\
\hline Se. 5 & 0 & 0 & 0 & 0 & 0 & 0 & 0 & 0 & 0 & 0 & 0 \\
\hline Se. 6 & 0 & 0 & 0 & 0 & 0 & 0 & 0 & 0 & 0 & 0 & 0 \\
\hline Se.7 & 0 & 0 & 0 & 0 & 0 & 0 & 0 & 0 & 0 & 0 & 0 \\
\hline Se. 8 & 1 & 30 & 2 & 1 & 30 & 2 & 1 & 30 & 2 & 2.0 & 2 \\
\hline Se. 9 & 0 & 0 & 0 & 0 & 0 & 0 & 0 & 0 & 0 & 0 & 0 \\
\hline Se.10 & 0 & 0 & 0 & 0 & 0 & 0 & 0 & 0 & 0 & 0 & 0 \\
\hline Se.11 & 1 & 30 & 2 & 14 & 420 & 3 & 14 & 420 & 3 & 2.7 & 3 \\
\hline Se.12 & 0 & 0 & 0 & 0 & 0 & 0 & 0 & 0 & 0 & 0 & 0 \\
\hline Se.13 & 0 & 0 & 0 & 0 & 0 & 0 & 0 & 0 & 0 & 0 & 0 \\
\hline Se.14 & 0 & 0 & 0 & 0 & 0 & 0 & 0 & 0 & 0 & 0 & 0 \\
\hline Se.15 & 0 & 0 & 0 & 0 & 0 & 0 & 0 & 0 & 0 & 0 & 0 \\
\hline Se.16 & 0 & 0 & 0 & 0 & 0 & 0 & 0 & 0 & 0 & 0 & 0 \\
\hline Se.17 & 0 & 0 & 0 & 0 & 0 & 0 & 0 & 0 & 0 & 0 & 0 \\
\hline Se. 18 & 0 & 0 & 0 & 0 & 0 & 0 & 0 & 0 & 0 & 0 & 0 \\
\hline Se.19 & 1 & 30 & 2 & 0 & 0 & 0 & 12 & 360 & 3 & 1.6 & 2 \\
\hline Se.20 & 50 & 1500 & 3 & 13 & 390 & 3 & 10 & 300 & 3 & 3.0 & 3 \\
\hline $\mathrm{B} 1$ & 1 & 30 & 2 & 0 & 0 & 0 & 0 & 0 & 0 & 0.67 & 1 \\
\hline $\mathrm{B} 2$ & 0 & 0 & 0 & 0 & 0 & 0 & 0 & 0 & 0 & 0 & 0 \\
\hline B3 & 0 & 0 & 0 & 0 & 0 & 0 & 0 & 0 & 0 & 0 & 0 \\
\hline B4 & 2 & 60 & 2 & 0 & 0 & 0 & 0 & 0 & 0 & 0.67 & 1 \\
\hline WE1 & 0 & 0 & 0 & 0 & 0 & 0 & 0 & 0 & 0 & 0 & 0 \\
\hline \multicolumn{10}{|c|}{ Average degree of contamination } & 0.42 & \\
\hline
\end{tabular}

Footnotes for tables $15-17$ :

$\mathrm{Ca}=$ SHG Carolinensiel

$\mathrm{Gä}=$ Gägelow

$\mathrm{Se}=$ Semlow 
For the 82 potato specimens tested in 2000 (see Table 17) an estimation of "suspected" contamination was reported by Stöver Co. on the basis of field observations and other data (see chapter 3.7.1).

A comparison of these two independent scorings of latent contamination is shown by Table 18 . Only 6 specimens were suspected to be contaminated by Stöver Co. Five of these were, indeed, found to be contaminated by erwinias in the laboratory tests in Göttingen. In additional 15 specimens, which were suspected to be free of contamination by Stöver Co., erwinias were detected in Göttingen, however with a low degree in most of the cases (5 very weak, 8 weak). All the other specimens were scored as free of contamination in both independent assessments.

Table 18: Comparison of latent contamination determined in Göttingen and suspected contamination estimated by Stöver Co. from field observations of potatoes harvested in 2000

\begin{tabular}{|c|c|c|}
\hline Sample no. & Laboratory tests, Göttingen & Field observations, Stöver Co. \\
\hline Se. 4 & weak & free \\
\hline Se. 8 & weak & free \\
\hline Se. 11 & moderate & moderate \\
\hline Se. 12 & free & moderate \\
\hline Se. 19 & weak & free \\
\hline Se.20 & moderate & moderate \\
\hline Gä 01 & very weak & free \\
\hline Gä 04 & moderate & free \\
\hline Gä 05 & weak & very weak \\
\hline Gä 06 & moderate & moderate \\
\hline Gä 08 & weak & free \\
\hline $\mathrm{Ba} 01$ & very weak & moderate \\
\hline $\mathrm{Ba} 04$ & very weak & free \\
\hline $\mathrm{Ca} 05$ & weak & free \\
\hline $\mathrm{Ca} 06$ & weak & free \\
\hline $\mathrm{Ca} 09$ & very weak & free \\
\hline $\mathrm{Ca} 27$ & weak & free \\
\hline Ca 30 & very weak & free \\
\hline
\end{tabular}




\subsubsection{Evaluation of potato lots with different degrees of latent contamination}

\section{by field experiments in the following year}

The potato lots with degrees of latent contamination by Ecc of 1 (weak), 2 (moderate) and 3 (strong) were all cultivar Morene harvested in 1998, but originated from Semlow 18 ha, Gägelow, and Semlow 33 ha, respectively. These potato lots were analysed for latent contamination in Göttingen in 1998. The results can be drawn from Table 15, as follows:

\begin{tabular}{|c|c|c|}
\hline Sample no. & Designation in 1998 & Grading in 1998 \\
\hline 1 & Se 2 & 1.0 \\
\hline 2 & Se 4 & 2.3 \\
\hline 3 & Se 5 & 2.7 \\
\hline
\end{tabular}

In the following vegetation period (1999) these potatoes were grown in an experimental design with 4 repetitions (subchapter 2.5) on a farm in Natenstedt. The results (Table 19) clearly show a higher tuber yield and higher tuber size in the variant 1 originating from weakly contaminated seed potatoes. The lowest total tuber yield was recorded in the variant 3 originating from strongly contaminated seed potatoes. Although the differences between variants 1 and 3 were highly significant, the differences between strongly (3) and moderatly (2) contaminated potato tubers were insignificant. Also, the percentage of tubers with soft rot was significantly lower in variant 1 than in variants 2 and 3 (BRUER, Stöver Co., person. communication).

Table 19: Effects of different degrees of latent contamination of seed potato lots with $E c c$, as determined in laboratory tests in Göttingen, on yield, tuber sizes and tuber numbers in different fractions according to tuber size

\begin{tabular}{|c|c|c|c|}
\hline Yield and tuber size & \multicolumn{3}{|c|}{ Seed potato lots with latent contamination } \\
\hline Yield in t/ha & $1:$ weak & $2.3:$ moderate & $2.7:$ strong \\
\hline Fraction $35-40$ & 1.82 & 2.1 & 2.06 \\
\hline Fraction $40-50$ & 17.69 & 17.2 & 17.08 \\
\hline Fraction $50-55$ & 13.19 & 15.4 & 11.58 \\
\hline Fraction 55 -60 & 11.94 & 10.1 & 10.83 \\
\hline Fraction $60+$ & 16.32 & 9.5 & 11.83 \\
\hline Total & $\mathbf{6 0 . 9 5}$ & $\mathbf{5 4 . 3}$ & $\mathbf{5 3 . 4}$ \\
\hline Tuber size & \multicolumn{4}{|}{} \\
\hline Number of tubers/10 kg & 54 & 62 & 54 \\
\hline Fraction 50+ & 44 & 49 & 44 \\
\hline Fraction 55+ & 36 & 41 & 36 \\
\hline Fraction $60+$ & \multicolumn{4}{|l}{}
\end{tabular}


Contamination with E. carotovora is very common in potato stocks and often becomes important during long storage periods when soft rot is caused (PEROMBELON et al., 1979). Soft rot of tubers in storage can be found in most enviromental conditions as major cause of infection of seed potatoes. It has also been clearly established that daughter tuber contamination may occur in the soil by spread of erwinias from rotting mother tubers and equipment used for harvesting and handling (GRAHAM and HARDIE, 1971). Therefore, the use of roguing has still an important place in controlling potato seed quality. In addition, seed quality can be improved substantially by determining Erwinia spp. levels on potato tubers (TOTH et al., 1996). Therefore, the most important measure to reduce losses by Erwinia carotovora soft rots is to eradicate or reduce the number of contaminated tubers during longtime storage and in seed potatoes. Also, potato genotypes have been screened for tuber resistance to erwinias and differences in resistance have been found (MUNZERT \& HUNNIUS, 1980; LAPWOOD et al., 1984). However, the degree of resistance is rather low in most of the cases. Several methods have been developed to quantify the number of erwinias on potato tubers. However, the sensitivity of these methods is often not satisfactory. Therefore, these studies aimed to improve the methods for detection of Erwinia spp. in potato stocks.

\subsection{Identification and differentiation of Erwinia spp.}

Three different soft rot erwinias are commonly associated with potatoes, Erwinia carotovora subsp. atroseptica (Eca) (van Hall 1902) Dye 1969, Erwinia carotovora subsp. carotovora (Ecc) (Jones, 1991) Bergey, Harrison, Breed, Hammer \& Huntoon, 1923, and Erwinia chrysanthemi (Ech) BURKHOLDER et al., all of which can cause tuber soft rot (PEROMBELON 1992). Erwinia cells in general are identified by their ability to form cavities on the semi-selective medium due to breakdown of polypectate by pectolytic enzymes. Two methods are available to differentiate between Eca and Ecc. The older one is based on the incubation at differential temperatures (PEROMBELON and HYMAN, 1986) and evaluation of cavity formation (PEROMBELON, 1992); the second method is the growth on the $\alpha$ methyl-d-glucoside medium. The results obtained in our studies revealed that Eca is able to form characteristic cavities already after $24 \mathrm{~h}$ at $27{ }^{\circ} \mathrm{C}, E c c$ does so at $27{ }^{\circ} \mathrm{C}$ and $35.5{ }^{\circ} \mathrm{C}$, whilst Ech forms cavities at $27^{\circ} \mathrm{C}, 35.5^{\circ} \mathrm{C}$ and $37^{\circ} \mathrm{C}$ within $24 \mathrm{~h}$. On $\alpha$-methyl-d-glucoside the bacterial colonies of Eca showed a pink colour, whereas weak white colonies were obtained by Ecc and Ech on the same medium after incubation at $27^{\circ} \mathrm{C}$ for $24 \mathrm{~h}$. Both tests gave good results in these studies in differentiation of several strains from the GSPB culture 
collection. The test on $\alpha$-methyl-d-glucoside was very safe to discriminate Eca from Ecc and Ech. However, it was difficult to distinguish Ecc and Ech by growth at 35.5 and $37^{\circ} \mathrm{C}$, as was also reported by JANSE and SPIT (1989). It appeared easier to differentiate Ecc and Ech by the phosphatase test, as was also shown by SHEKHAWAT and RUDOLPH (1978) after protein electrophoresis.

\subsection{Preservation of Erwinia spp. strains used in this study}

Several culture preservation methods were tested and evaluated for their suitability in maintaining the viability of Erwinia spp.. Our experiments showed that a very suitable method for preservation of Erwinia spp., Eca and Ecc was incubation in sterile distilled water at room temperature. Thus, the viability of all Erwinia spp. was maintained for more than 16 months at $4{ }^{\circ} \mathrm{C}$ as well as at room temperature. This method is very simple, and the bacterial strains did not lose virulence during storage. BDLIYA (1995) reported that all strains of Erwinia spp. were preserved at $18{ }^{\circ} \mathrm{C}$ in calcium carbonate based liquid medium (YPC) in tightly closed screw capped test tubes.

\subsection{Development of a semi-selective medium for isolation of soft rot erwinias}

Specific media for isolation of Erwinia spp. from potato tubers and identification of the pathogen have been in use for a long time. Several selective media containing pectin or sodium polypectate were developed with different degrees of success for detection of soft rot bacteria from potatoes (STEWART, 1962; LOGAN, 1963; BERAHA, 1968; PEROMBELON, 1971; THORNE, 1972; CUPPELS and KELMAN, 1974; COTHER et al., 1980; BDLIYA 1995). The isolation of Erwinia spp. using the selective media is based on the ability of the pathogen to hydrolyze polypectate and to form typical cup-shaped pits (cavities) in the medium. As was shown by PEROMBELON (1971), other pectolytic bacteria occurring in the soil, especially certain pseudomonads, also form cavities, but these are shallow and quite distinct from those caused by varieties of E. carotovora, which are easily recognized even when there is overcrowding by other organisms. CUPPLES and KELMANN (1974) developed the crystal violet pectate based selective medium (CVP) for isolation of erwinias dependent on the quality of the polypectate used (PIERCE \& McCAIN, 1992). PEROMBELON and BURNETT (1991) described two crystal violet polypectate media for detection of soft rot erwinias. These were a single layer (CVPB) and a double layer (DLCVP) medium based on the sodium polypectate "Bulmer". The authors found that the growth of Erwinia spp. on the CVPB medium was poor and they suggested that the tryptone probably chelates heavy metals present in the "Bulmer" 
pectin preparation rather than acting as a nutrient source. The adding of 0.1 or $0.5 \%$ tryptone to the selective medium resulted in growth and cavity formation. The effect of increasing the concentration of tryptone on the sensitivity of the selective-medium was therefore evaluated by PEROMBELON and BURNETT (1991). BDLIYA (1995) also modified the double layer crystal violet mediun for isolation of Erwinia spp. by using the sodium polypectate "Bulmer" and found that the addition of tryptone to the basal and over layer improved the cavity formation by soft rot erwinias.

Our studies revealed that without tryptone and citrate bacterial colonies with cavities did not develop, and only few colonies and weak cavities were formed when tryptone and citrate were added at low concentrations to the basal and over layer, respectively. On the other hand, many colonies grew when tryptone $(0.6 \%)$ was added only to the basal layer and trisodium citrate dihydrate $(0.5 \%)$ only to the overlayer. It was concluded from these results that tryptone diffusing from the basal layer served as nitrogen source for the bacteria in the overlayer and promoted their growth, whereas concomitant presence of tryptone and pectin in the overlayer did not force the bacteria to utilize pectin as carbon source. Obviously, the additional carbon source citrate enhanced bacterial growth in the overlayer without reducing pectin degradation by the bacteria. Thus, the addition of tryptone to the overlayer and of citrate to the basal layer was not necessary.

With the original amount of $18 \mathrm{~g}$ polypectate in the overlayer the medium became too solid and only weak cavities were obtained, whereas the reduction to $15 \mathrm{~g}$ polypectate per litre allowed very good cavity formation. Omitting of bromothymol blue resulted in more distinctive cavities because the medium of BDLIYA with two indicators (bromothymol blue and crystal violet) became green, so that the appearance of the cavities was not clear. The reduction of the amount of $\mathbf{C a C l}_{2} \times 2 \mathrm{H}_{2} \mathrm{O}(10 \%)$ in the overlayer, compared to the medium of BDLIYA (1995), slightly reduced the firmness of this medium and improved the growth and cavity formation of Erwinia spp., whereas without $\mathrm{CaCl}_{2} \times 2 \mathrm{H}_{2} \mathrm{O}(10 \%)$ the medium did not solidify. Also in planta, the formation of calcium-pectate complexes appears to be responsible for increased resistance, as it may protect the tissue against enzymatic degradation by the pathogen (BATEMAN and MILLAR, 1966; PAGEl and HEITEFUSS, 1989).

\subsection{Comparison of different pectin sources for detection of Erwinia spp. on the semi-selective medium}

Several commerical pectin and polypectate preparations were used in these studies and found to be unsatisfactory for isolation of Erwinia spp. from potatoes because some did not solidify 
at $\mathrm{pH} 7.2$ or did not allow satisfactory growth of erwinias. This may have been due to high amounts of heavy metals which are toxic to Erwinia spp. (PEROMBELON \& BURNETT, 1991).

From our studies we concluded that there is a strong relationship between the suitability of the pectins and degree of esterification. All the Erwinia spp. produce several pectolytic enzymes two of which play a major role. The first is pectinmethylesterase (PME), and the second pectintranseleminase (pectate lyase) (PL). PL is produced in higher activity than PME by erwinias (MORAN and STARR, 1969; GARDNER and KADO, 1976; CHATTERJEE et al., 1979; FAVEY et al., 1992). Since PL cleaves internal glycosidic linkages by ß-elimination after demethylation of the polymer by pectin methylesterase, PL causes a rapid decrease in the viscosity of polygalacturonic acid (GLENN, 1976; BRAUN and SCHMITZ, 1980; FAVEY et al., 1992) but not of pectin. Also, an increase in pectin esterification (more than $20 \%$ of galacturonic acid residues methoxylated) leads to less efficient degradation of the substrate by PL (FAVEY et al., 1992). PAGEl and HEITEFUSS (1990) found that $14 \mathrm{~h}$ after inoculation of potato tubers with Eca the main enzyme activity was PG, whereas after $22 \mathrm{~h}$ PL played the dominant role. Therefore, the growth of Erwinia spp. and cavity formation was good on pectin with a low degree of esterification, such as pectic acids from apple (H.+ C.) and citrus (H.+ C.), Copenhagen (A/S) pectin and pectin N (Roth), that had an esterification degree of 5, 7, $<10 \%$ and $8 \%$, respectively. On the other hand, pectins with high esterification did not show clear cavities, such as pectin A (Roth), pectin C (Roth), pectin from citrus (Serva), pectin from citrus (Fluka), and pectin from apple (H.+ C.) with degrees of esterification of 36\%, 67-71\%, $65 \%, 63-66 \%$ and $32 \%$, respectively, (see 2.3.3). It is suggested, therefore, that future tests with pectins suited for detection of erwinias should select preparations with a low esterification degree. It appears as if the content of heavy metals, as suggested by PEROMBELON and BURNETT (1991), does not play a decisive role for the suitability of pectins in the semiselective medium.

\subsection{Recovery rate and detection limit of erwinias in artificially contaminated potato homogenates}

The recovery of Erwinia spp. strains on CVPM were $87 \%$ compared to $49 \%$ and $23 \%$ on the media of BDLIYA (1995) and PEROMBELON \& BURNETT (1991), respectively. As reported by PEROMBELON \& BURNETT (1991) 7 from 12 strains showed a recovery rate of $<50 \%$ on CVPB compared to NA. 
From our studies it can be concluded that the detection limit on the new semi-selective medium was 2 X $10^{1} \mathrm{cfu} / \mathrm{ml}$. Also BDLIYA (1995) reported that the detection limit on his medium was $10^{2} \mathrm{cfu} / \mathrm{ml}$, whereas the dilution platings on a selective medium of PEROMBELON and HYMAN (1986) allowed detection of $1-5 \times 10^{4} \mathrm{cfu} / \mathrm{ml}$ peel extract (JONES et al., 1994). VAN VUURDE and ROOZEN (1990) reported a detection limit of Ecc and Eca at levels of $10^{3} \mathrm{cfu} / \mathrm{ml}$ of slurry by isolation on CVP. The low detection limit determined in our studies may be due to some modifications in the semi-selective medium of Bdliya (1995) as described in subchapter 4.3 .

Thus, the modified semi-selective medium (designated CVPM) has several advantages compared to other selective media: 1) The detection limit of soft rot erwinias from potatoes was $\left.2 \times 10^{1} \mathrm{cfu} / \mathrm{ml}, 2\right)$ the ability of Erwinia spp. to produce the cavities were distinctive after $13 \mathrm{~h}$ of incubation at $27{ }^{\circ} \mathrm{C}$ (AHMED et al., 2000), 3) erwinias can be detected in the concomitant presence of high populations of other saprophytic bacteria, and 4) the two soft rot erwinias commonly associated with potatoes $(E c c$ and $E c a)$ can be differentiated by cultivation at different temperatures and by transferring directly from the cavities to the $\alpha$-methyl-dglucoside medium.

Previous work has shown that standard serological methods such as immunofluorescence staining and the double antibody sandwich enzyme linked immunosorbant assay (DAS)(ELISA) are not suitable to quantify E.c. contamination of potato tubers, partly because of low sensitivity of $10^{5} \mathrm{cfu} / \mathrm{ml}$ (JONES et al., 1994), and because the quantitative methods do not distinguish clearly between different subspecies (JONES et al., 1993). By using the new semi-selective medium (CVPM) the potato seed quality can be improved substantially by determining latent contamination with E. carotovora on tubers. However, a number of different methods for enumeration of E. carotovora on the tuber stocks are available and are continuously by being improved, such as immunofluorescence and DAS-ELISA (VAN VUURDE \& ROOZEM, 1990; TOTH et al., 1996; ALLEFS et al., 1995; HELIAS et al., 2000).

\subsection{Occurrence of different erwinias}

The morphological and biochemical tests applied to differentiate erwinias in these studies confirmed the taxonomic identification of several Eca, Ecc and Ech strains from the GSPBbacterial collection. Although all the potato specimens tested looked healthy, a latent contamination with Erwinia carotovora spp. carotovora was determined in $2 / 3$ (10 from 16) of the samples purchased from shops. Also, the cultivars showed differences in degree of contamination by Erwinia spp. according to the contamination level on the tubers. 
Interestingly, all the bacterial strains isolated from potato tubers purchased from different shops and supermarkets in this study (99 isolates) were identified as Ecc.

Earlier studies concluded that the tubers obtained from apparently blackleg free tubers were contaminated with both, Eca and Ecc (PEROMBELON, 1972; PEROMBELON and KELMAN, 1987). Similar results were obtained in Germany (Van den BOOM, 1967; FICKE et al., 1973; NAUMANN et al., 1976); and in USA (De BOER and KELMAN, 1975; NIELSON, 1978), where the relative proportion of $E c a$ and $E c c$ varied widely on the tubers. PEROMBELON (1971) reported that more Eca cells (4 X) than Ecc were obtained from tubers taken from storage. In contrast, $90 \%$ of the isolates from naturally rotting tubers in storage were Ecc in studies of LAZAR and BUCUR (1964). None of these workers reported the presence of Ech. Although Eca is the predominant agent involved in blackleg under cool climates, Ecc occurs in both temperate and warm climates but mostly in storage, and Ech predominates in warm regions as a storage pathogen (PEROMBELON and KELMAN, 1987; PEROMBELON and HYMAN, 1989; SALMOND, 1992; HELIAS et al., 2000). The two pathogens $E c c$ and Eca can attack the tubers in the field and after harvest, and Ech may also be an important cause of decay of seed potatoes at soil temperatures in the region of $30{ }^{\circ} \mathrm{C}$ (LELLIOTT, 1974; LUND, 1979; COTHER, 1980 and SKERMAN et al., 1980).

According to our results all isolates obtained from tubers originating from potato stocks were Ecc. Although these results confirmed those reported by LAZAR and BUCUR (1964) that $90 \%$ from the erwinias were Ecc, they differ from several other reports as listed above. The conflicting results may be due to many factors such as anaerobic conditions prevailing during storage, free water covering the tuber surface, temperatures above the minimum required for growth of the pathogen, and physiological factors such as a high water potential, all of which favour infection by Ecc (CROMARTY and EASTON, 1973; KELMAN et al., 1978).

The non-detection of $E c a$ in potato stocks in our studies may be due to the fact that the potato lots were assessed after several months of storage. Thus, SCHOBER and ZADOKS (1999) reported that initial numbers of $E c a$, obtained by artificial infection, decreased rapidly during the first weeks of storage and did not increase significantly later in the storage period. Similarly, Van VUURDE \& De VRIES (1994) found a continuous decrease of Eca populations on the surface of potato tubers during storage.

Another possibility is that the new semi-selective medium favours the growth of Ecc more than that of Eca, because EL-KAZAZZ (1984) found that generally Ecc strains showed higher activities for pectate lyase (PL) in vitro and in vivo than Eca. It was also observed in our studies with pure cultures that cavities of Ecc strains on the semi-selective medium were broader and deeper than those of Eca (chapter 3.5.1). Therefore, the possibility can not be 
excluded that contaminations by Eca were overlooked in some of the samples because the cavities produced were much smaller than those by Ecc. On the other hand, field experiments on the effect of Degaclean on infection of potatoes by erwinias clearly showed that also Eca contamination was detected by King B medium which was supplemented with rifampicin (chapter 2). Further extensive experiments have to find out whether the sensitivity of the CVPM medium to detect contamination with Ecc and Eca under natural conditions differs.

\section{7}

\section{Evaluation of methods to identify and quantify latent contamination of potatoes by erwinias}

In our studies two methods were tested for isolation and detection of soft rot causing erwinias from potatoes. The results obtained showed that higher numbers of Erwinia spp. colonies were obtained from potato tubers by the soaking method compared to potato tuber peel homogenates (Table 9). It was shown earlier by PEROMBELON (1973) that often the tuber surfaces are contaminated by erwinias. The high proportion of latent contaminations in collective tuber samples at harvest can probably be explained by the fact that collective samples have a higher probability of being contaminated by at least one contaminated tuber, but may also reflect the higher contamination rates of small tubers (HELIAS et al., 2000). It is assumed that nearly all the bacteria on the potato tuber surfaces were isolated by the soaking method. This method also needed less time than analyzing homogenates from potato peels. Soaking of tubers in $0.01 \mathrm{M}$ $\mathrm{MgSO}_{4}$ for $3 \mathrm{~h}$ may allow nearly all Erwinia spp. contaminants to diffuse from the potato tubers into the soaking solution, because tuber contamination by erwinias is usually on the tuber surface. Soaking for only $3 \mathrm{~h}$ did not allow the bacteria to multiply because the temperature was very low $\left(4^{\circ} \mathrm{C}\right)$. In 10 from 11 samples tested, lower numbers of erwinias were obtained from peel homogenates than from soakates, and in one case with very low contamination bacterial colonies were only obtained from the soakage. Thus, our results confirmed those obtained by PEROMBELON et al. (1998) who also explained that tuber contamination by erwinias is usually superficial, having originated from infected rotting mother and progeny tubers in the field and during mechanical handling after harvest (PEROMBELON, 1992).

\subsection{Sample size}

When quantifying E. carotovora contamination of a potato stock, the tuber sample size should be representative, taking into account the variation in tuber contamination level that can be expected. The numbers of bacteria vary from tuber to tuber (PEROMBELON, 1972; 
PEROMBELON et al., 1998), and it is practice to take between 25 and 70 tubers randomly per stock depending on how the tubers are stored, and the expected level of contamination. According to our results (Tables 12 and 13), for quantifying Erwinia spp. contamination of a potato stock a representative sample size should consist of at least 20 tubers taken randomly. Depending on the size of the potato stock, this test should be repeated at least three times with 20 potato tubers in each repetition.

\subsection{Comparison of different years}

Contamination with E. carotovora ( $E c a$ and $E c c$ ) was very common in stored seed potato stocks classified as "Elite" seed or commercial seed (HARJU and KANKILA, 1993). However, contamination levels were highest after a rainy growing season (HARJU and KANKILA, 1993). In our studies, the semi-selective medium (CVPM) was tested on 232 samples from Stoever Co. from three vegetation periods (1998, 1999 and 2000). The results obtained (Tables 15, 16 and 17 ) showed a considerable variation in bacterial contamination from "very weak" to "very strong". The lowest contamination was determined for the potatoes harvested in 2000, and the highest contamination occurred in 1998. Thus, the average degrees of contamination were $2.13,1.24$ and 0.42 for the years 1998, 1999 and 2000, respectively.

Table 20: Rainfall in mm from April - Septemper

\begin{tabular}{|c|c|c|c|c|c|c|c|c|}
\hline \multirow[b]{2}{*}{ Location } & \multirow[b]{2}{*}{ Year } & \multicolumn{6}{|c|}{ Months } & \multirow[b]{2}{*}{ Total } \\
\hline & & April & May & \begin{tabular}{|l|} 
June \\
\end{tabular} & July & August & Sept. & \\
\hline \multirow[t]{3}{*}{ Carolinensiel } & 00 & 32.0 & 74.0 & 79.0 & 62.0 & 53.0 & 60.0 & 360 \\
\hline & 99 & 33.0 & 17.0 & 51.0 & 63.0 & 73.0 & 65.0 & 302 \\
\hline & 98 & 107.0 & 28.0 & 102.0 & 96.0 & 104 & 86.0 & 523 \\
\hline \multirow[t]{3}{*}{ Semlow } & 00 & 24.0 & 44.0 & 93.0 & 90.0 & 85.0 & 59.0 & 395 \\
\hline & 99 & 55.0 & 43.0 & 117.0 & 39.0 & 89.0 & 38.0 & 381 \\
\hline & 98 & 62.0 & 49.0 & 77.0 & 144.0 & 95.0 & 46.0 & 473 \\
\hline \multirow[t]{3}{*}{ Gägelow } & 00 & 27.0 & 35.0 & 55.0 & 116.0 & 106.0 & 61.0 & 400 \\
\hline & 99 & 21.0 & 87.0 & 70.0 & 24.0 & 47.0 & 48.0 & 297 \\
\hline & 98 & 73.0 & 23.0 & 140.0 & 99.0 & 57.0 & 24.0 & 416 \\
\hline
\end{tabular}

Obviously, the high degree of contamination by erwinias in 1998 was due to higher rainfall in this year compared to 1999 and 2000 (Table 20) and to wet conditions during harvest in 1998 (Bruer, Stöver Co., person. communication). The same correlation existed when the potato 
samples of the three locations Carolinensiel, Semlow and Gägelow were compared for the year 2000. Thus, rainfall in August with 53,85 and $106 \mathrm{~mm}$ corresponded to average contamination degrees of $0.26,0.6$ and 1.1, respectively (Table 17).

\subsection{Comparison of field observations and laboratory tests}

Two methods for assessing latent contamination by erwinias were compared, field observations by Stöver Co. and laboratory tests in Göttingen, with 94 samples of potato tubers in two years (1998 and 2000). The scoring of the same potato provenances by experts from Stöver Produktion Co. in the 3 grades (weak, moderate and strong) was based on the following criteria: visible appearance, knowledge on susceptibility of different cultivars, optical impression of field plants, and conditions on the field, such as soil type and soil humidity. Thus, the grading by Stöver company was completely independent from the laboratory tests in Göttingen and only based on the experience of the experts.

In the year of 199812 specimens of healthy looking potato tubers were assessed. The laboratory tests in Göttingen (Table 14) showed a considerable variation in bacterial contamination from "very weak" to "very strong", but these scorings were very similar to those by Stöver Co.. Only in 2 form 12 samples the results differed markedly, in that the potatoes were found to be very strongly contaminated by erwinias in laboratory tests in Göttingen, but assessed as moderately affected by Stöver Co. experts.

In the year of 2000, a comparison of these two independent assessments of latent contamination of 82 specimens showed that only 6 specimens were suspected to be contaminated by Stöver Co., and five of these were, indeed, found to be contaminated by erwinias in the laboratory tests in Göttingen. In additional 15 specimens, which were suspected to be free of contamination by Stöver Co., erwinias were detected in Göttingen, however with a low degree in most of the cases ( 5 very weak, 8 weak). All the other specimens were scored as free of contamination in both independent assessments.

The comparison of field observations and laboratory tests in the two years, thus, revealed 1) a rather good agreement between both assessments, and 2) a higher sensitivity of the microbiological tests in Göttingen.

The microbiological assay can be affected by variations from tuber to tuber and also from stock to stock. Thus, if only one strongly contaminated tuber was contained in the sample the whole sample would be scored as contaminated. Also JONES et al. (1994) found that the tubers taken from the surface of a stock or box tended to be less contaminated than those from below the surface. Because of the rather good correspondence of the two assessments in most of the cases 
Stöver Produktion Co. agreed to continue the cooperation with the Department of Plant Pathology and Plant Protection in Göttingen to classify the potato stocks for contamination level by soft rot causing Erwinia spp.. This successful cooperation was only possible by applying the new semi-selective medium which is particularly well suited for detecting Erwinia carotovora ssp. carotovora, the major causal agent of potato soft rot during storage.

\section{11 Field experiments with potato lots assessed in different degrees of latent contamination by $E c c$}

These experiments (see Chapter 3.7.3) clearly revealed that potato lots assessed as lowly contaminated by Ecc (degree 1) gave higher yields in the following vegetation period with higher tuber sizes and a lower percentage of tubers with soft rot than potato lots assessed in contamination degrees 2.3 (moderate) and 2.7 (strong). As shown in Table 19, the yields from seed potatoes which were graded as moderately or strongly contaminated by Erwinia carotovora did not differ significantly with 54.3 and $53.4 \mathrm{t} / \mathrm{ha}$, respectively. This was not surprising because also the gradings for latent contamination with Erwinia carotovora showed only slight differences with 2.3 and 2.7 , respectively. On the other hand, the seed potatoes graded as weakly contaminated (grade 1.0) gave a higher yield (60.95 t/ha) in the following year than the other two samples.

Therefore, potato lots assessed as lowly or non-contaminated by Erwinia carotovora in the microbiological tests appear to be best suited for long storage and should as far as possible be preferred for potato production in the following year, because yield and quality could be improved.

The finding that potatoes originating from the lot with contamination degree 2 (moderate) contained significantly smaller tubers than the other two variants at harvest, may be due to the fact that this was the only potato lot originating from Gägelow and not from Semlow. Some observations indicated that the potatoes grown in Gägelow were stronger affected by virus diseases than those in Semlow (BRUER, person. communication). 


\section{SUMMARY}

1) The objective of this study was to detect latent contamination of potato tubers by soft rot causing erwinias [Erwinia carotovora subsp. atroseptica (Eca), and Erwinia carotovora subsp. carotovora $(E c c)]$ and to improve the methods for isolation and identification.

2) All the Erwinia strains used in this study were clearly identified and differentiated by the classical tests, including pectolytic activity on potato tuber slices, utilization of $\alpha$-methyl-dglucoside, production of reducing substances from sucrose, phosphatase activity, growth on Logan's medium, and indole production. Virulence of the strains was tested by bioassay on potato tuber slices.

3) Testing several methods for preservation of Erwinia spp. revealed that the Erwinia strains (Eca, Ecc and Erwinia chrysanthemi; Ech) maintained viability for at least 16 months when preserved in sterile distilled water at $4{ }^{\circ} \mathrm{C}$ as well as at room temperature.

4) For a sensitive detection and identification of soft rot erwinias, the semi-selective double layer medium of Bdliya (1995) was modified. The amount of sodium polypectate was reduced from $18 \mathrm{~g}$ to $15 \mathrm{~g}$ per litre, tryptone and bromothymol blue were omitted from the overlayer, and the amount of $\mathrm{CaCl}_{2} \times 2 \mathrm{H}_{2} \mathrm{O}(10 \%)$ was reduced.

5) Most crucial for the modified medium, called CVPM, was the pectin source. When 15 pectin preparations were tested, only 5 allowed the formation of typical cavities by erwinias. Obviously, the degree of esterification was decisive. Thus, the growth of Erwinia spp. and cavity formation was much better on the CVPM medium with pectic acids with a low esterification degree $(5-9 \%)$ than with pectins with a high degree of esterification (32-71\%).

6) The recovery rate of bacteria from artificially inoculated tuber sap on the new semiselective medium was ca. $\mathbf{9 0 \%}$ in comparison to other semi-selective media, where the recovery rate was $49 \%$ for CVPD (BDLIYA, 1995) and only $23 \%$ for the medium of PEROMBELON \& BURNETT (1991). Therefore, the detection limit by using the CVPM 
medium was $2 \times 10^{1} \mathbf{c f u} / \mathbf{m l}$.

7) In order to detect latent contamination of potatoes with Erwinia spp. the tubers were soaked in $0.01 \mathrm{M} \mathrm{MgSO}_{4}$ for $3 \mathrm{~h}$ at $4{ }^{\circ} \mathrm{C}$. The soakage was concentrated by centrifugation.

8) For quantifying Erwinia spp. contamination of potato stocks a representative sample size should consist of at least 20 tubers taken randomly. Depending on the size of the potato stock, this test should be repeated at least three times with 20 potato tubers in each repetition.

9) By application of this procedure 99 strains were obtained from different shops, supermarkets and farms. In $2 / 3$ of the specimens erwinias were isolated from healthy looking potato tubers and all of the isolates were identified as Ecc.

10) The semi-selective medium (CVPM) was tested on 232 potato specimens obtained from Stoever Produktion GmbH \& Co. KG and harvested in the years 1998, 1999 and 2000. These tests revealed considerable differences between the samples. In general, the specimens from the year of 1998 were much stronger contaminated than those of the years 1999 and 2000. The lowest contamination was determined for the potatoes harvested in 2000.

11) In two cases (12 and 82 potato specimens harvested in 1998 and 2000, respectively) a comparison of the results obtained by isolation of erwinias with the semi-selective medium and field observations from Stoever Co. showed a very good correspondence of the determined and suspected contamination by Erwinia carotovora. It was concluded that the CVPM medium is well suited for isolation of Erwinia spp. (particularly for Ecc) from latently contaminated potatoes.

12) Field experiments proved that seed potato lots assessed as moderatly or strongly contaminated by Ecc gave lower yields and less quality than potato lots assessed as weakly contaminated. Therefore, the microbiological assessment of potato lots for latent contamination by Erwinia carotovora appears to be very valuable for improving yield, quality and storage capability of potatoes. 


\section{CHAPTER II}

\section{Effect of Degaclean ${ }^{\circledR} 150$ on the reduction of soft rot of potato tubers}

\section{INTRODUCTION}

One of the economically most important bacterial pathogens of plants and plant products is Erwinia carotovora. Especially during storage of potatoes high losses can occur. In contrast to fungal pathogens, the erwinias, like most other phytopathogenic bacteria, cannot be controlled by specific bactericides. Antibiotics are rather expensive and are not allowed in most of the cases, because of the risk that an application of antibiotics on a broad scale may give rise to antibiotic resistant bacterial populations which may transfer the resistance gene(s) to human pathogenic bacteria. Other bactericidal compounds, such as copper or sulphur-containing formulations, are not very effective and cause environmental pollution.

Therefore, several other measures have been developed to reduce bacterial diseases and postharvest losses (OKON, 1990). In the case of Erwinia carotovora these measures include, for instance, crop rotation, optimal fertilization, careful irrigation, and especially several precautions during harvest and storage of potatoes.

Another alternative of directly eradicating erwinias is the application of oxidizing agents which are not harmful to the environment. The toxicity of oxygen against microorganisms is long known (GOTTLIEB, 1971). Thus, hydrogen peroxide is known since many years as disinfectant to inactivate or kill microorganisms. Peracetic acid is being used as disinfecting compound in human and veterinary medicine as well as in paper and cellulose industry, bottling industry and in food markets.

GREGORY and FRIDOVICH (1973) studied the mode of action and concluded that $\mathrm{O}^{*---}$ is one of the active agents of the peroxid. The second active component is the hydroxyl radical $\left(\mathrm{OH}^{*}\right)$. The extreme reactive radical $\mathrm{O}^{*---}$ oxidizes very fast diverse cell components, such as unsaturated fatty acids. Because these reactions occur so fast and unspecifically, allmost all microorganisms are killed after short exposure times (ROTILIO et al., 1973). The advantages of using hydrogen peroxide and peracids are twofold. Because of the fast and undirected effect the probability of microorganisms to become resistant is low. Secondly, these substances disintegrate after short reaction-time into non-polluting compounds, such as water, gaseous oxygen and organic acids. Consequently, the environment is hardly endangered by application of peracids and hydrogen peroxide. 
Very short reaction-times are always of advantage when the microroganisms have to be killed quickly (PRIOR et al., 1998). Therefore, Degaclean ${ }^{\circledR}$ and Clarmarin ${ }^{\circledR}$ were developed by Degussa $A G$, Frankfurt. Degaclean ${ }^{\circledR} 150$ is a mixture of $<5 \%$ peracetic acid $\left(\mathrm{CH}_{3} \mathrm{COOOH}\right)$ and $20-<60 \%$ hydrogen peroxide. Earlier experiments of MIELKE and HOPPE (1982) revealed that peracetic acid applied to the soil affected several fungal pathogens causing foot rot diseases of cereals.

However, these products are not plant protectants, such as fungicides or bactericides, because due to their unspecificity they also damage living plants. But, they can be used as disinfectants and additions to washing processes. Thus, experiments of NIEPOLD (1999b) showed that application of peracids to sewage water of a starch processing factory was very effective in destroying all microorganisms, including the quarantine species Ralstonia solanacearum and Clavibacter michiganensis ssp. sepedonicus. A concentration of 2000 ppm Clarmarin/catalase inhibitor (1:1) killed all microorganisms while the traditional hydrogen peroxide preparation ("Perhydrol") affected vitality of the microbial populations only weakly.

Due to these positive effects, our studies aimed to find out whether the addition of Degaclean to the washing water of potato tubers or spraying of potato tubers would decrease latent contamination of potatoes by soft rotting erwinias. Therefore, laboratory as well as field experiments were carried out. Additionally, the potential of Erwinia carotovora strains to develop Degaclean-tolerance should be investigated and possible modes of action be characterized, such as changes in the LPS structure. 


\subsection{Selection of antibiotics resistant isolates from Erwinia spp.}

In order to facilitate the monitoring of Erwinia carotovora populations as affected by Degaclean-treatment of potato tubers, antibiotic-resistant strains were generated. This was achieved by subjecting the strains Erwinia carotovora spp. atroseptica (Eca) (GSPB no. 2967) and Erwinia carotovora spp. carotovora (Ecc) (GSPB no. 436) to increasing concentrations of the antibiotics Rifampicin (Fluka no. 83907) and Streptomycin (Sigma no. S-6501) (AL SHINAWI, 1996). The experiment started by culturing the bacteria in King's medium B (KB) (see chapter I, 2.1.2) for $24 \mathrm{~h}$. The bacterial growth was scraped off and inoculated into $50 \mathrm{ml}$ King's B liquid medium and again incubated at $27{ }^{\circ} \mathrm{C}$ for $24 \mathrm{~h}$. After that $5 \mathbf{~ p p m}$ of sterile filtrated Rifampicin (200 mg/10 ml methanol) and 25 ppm Streptomycin sulfate $(1.0 \mathrm{~g} / 10$ $\mathrm{ml}$ water) were added to separate bacterial cultures and incubated at $27^{\circ} \mathrm{C}$ for $24 \mathrm{~h}$. From each flask $0.1 \mathrm{ml}$ were streaked onto King's medium B. After incubation of plates at $27^{\circ} \mathrm{C}$ for $24 \mathrm{~h}$, bacterial growth was scraped off and inoculated into new flasks, incubated at $27{ }^{\circ} \mathrm{C}$ for $24 \mathrm{~h}$, and 10 ppm Rifampicin and 50 ppm Streptomycin sulfate were added. The flasks were incubated for $24 \mathrm{~h}$. In the same way, the concentrations of the antibiotics were increased stepwise until 200 ppm Rifampicin and 800 ppm Streptomycin sulfate. The finally obtained isolates with resistance against the antibiotics were lyophilized and stored in the GSPB culture collection with numbers 2968 (Eca resistant against 200 ppm Rifampicin) and 2970 (Ecc resistant against 200 ppm Rifampicin and 800 ppm Streptomycin sulfate).

\subsection{Selection of Degaclean tolerant isolates of Erwinia spp.}

The generation of two strains of Erwinia spp. (from Eca 2967 and Ecc 436) with tolerance against Degaclean was performed similar to generation of antibiotic resistant strains (subchapter 2.1, see above) by subjecting the bacteria to increasing concentrations of Degaclean. The bacterial strains were grown on Petri plates with King's medium B and incubated at $27^{\circ} \mathrm{C}$ for $24 \mathrm{~h}$, after which the bacterial growth from each strain was scraped off and in the first step was inoculated into Erylenmeyer flasks with King's medium B and incubated again at $27^{\circ} \mathrm{C}$ for $24 \mathrm{~h}$. Aliquots from the bacterial suspensions were serially diluted and $0.1 \mathrm{ml}$ were plated on King's medium B in order to estimate the bacterial concentration before Degaclean was added. In the second step $0.3 \%$ Degaclean was added, the Erylenmeyer flasks were incubated at $27^{\circ} \mathrm{C}$ for $12 \mathrm{~h}$, and serial dilutions were plated on King's medium B. New Erylenmeyer flasks with $50 \mathrm{ml}$ King's B liquid medium were then inoculated with $0.1 \mathrm{ml}$ 
from the flasks with $0.3 \%$ Degaclean, incubated at $27{ }^{\circ} \mathrm{C}$ for $24 \mathrm{~h}$, after which the Degacleanconcentration was raised to $0.6 \%$, followed by incubation at $27{ }^{\circ} \mathrm{C}$ for $12 \mathrm{~h}$, serial dilution and plating of aliquots. The same procedure was continued several times until 1.5\% Degaclean was reached. This concentration totally inhibited further bacterial growth.

\subsection{Inoculation of potato tubers with Erwinia spp. by wounding}

The bacterial strains Erwinia carotovora subsp. atroseptica (Eca) (GSPB nos. 9201, 2967 and 2968) and Erwinia carotovora subsp. carotovora (Ecc) (GSPB nos. 427, 429 and 436) were grown on King's medium B at $27^{\circ} \mathrm{C}$ for $24 \mathrm{~h}$, after which the growth was scraped off in 0.01 $\mathrm{M} \mathrm{MgSO}_{4}$ solution. Each strain was adjusted to an O.D.660 of 0.06 (photometer, Spectronic 20, Bausch \& Lomb), corresponding to ca. $2.2 \times 10^{8} \mathrm{cfu} / \mathrm{ml} E c a$ and ca. $2.6 \times 10^{8} \mathrm{cfu} / \mathrm{ml}$ for $E c c$. The three bacterial suspensions of each subspecies were mixed at the ratio of $1: 1: 1$. Potato tubers of cultivars Bintje, Morene and Cilena were wounded by a pinflowerholder ("fakirbed" no. 202, Ø $32 \mathrm{~mm}, \mathrm{ZÜRICH}$ ) which was turned on the tuber surface under slight pressure by hand. Potatoes were wounded before or after soaking in 1.5 litre of two bacterial concentrations (ca. $10^{8}$ and $10^{6} \mathrm{cfu} / \mathrm{ml}$ ) in buckets at room temperature for $3 \mathrm{~h}$. Then the potato tubers were placed on filter paper at room temperature for drying over night. For the laboratory experiments five tubers from each variant were incubated in plastic boxes (with moist paper at the bottom) at $27^{\circ} \mathrm{C}$ for 3-6 days to find out which inoculation method was best suited.

\subsection{Effect of Degaclean on potato tubers infected by Erwinia spp.}

\subsubsection{Origin of Degaclean}

Degaclean was specified as follows: Degaclean ${ }^{\circledR} 150$, stabilized mixture of $20-<60 \%$ $(\mathrm{w} / \mathrm{w})$ hydrogen peroxide and $<5 \%(\mathrm{w} / \mathrm{w})$ peroxyacetic acid.

A sample of $1 \mathrm{~kg}$ was obtained from

SVR, Lösungen für die Umwelt, Birgit Schneider,

Hanauer Landstr. 2, D-63594 Hasselroth, Tel. and Fax 06055-83873

by order of

Degussa-Hüls AG, Herrn Huss, Abt.: IC-BC, Weissfrauenstr. 9, D-60311 Frankfurt/Main 


\subsubsection{Laboratory experiments}

Potato tubers were inoculated as described above (2.3). Potato samples of 5 tubers each were immersed in $0.5 \%, 1.0 \%$ or $2.0 \%$ Degaclean for different time intervals (15, 30 or $60 \mathrm{~min})$. Each variant was repeated twice. Finally, the potato tubers of each variant were placed on wet filter paper in plastic boxes $(19 \mathrm{~cm}$ X $19 \mathrm{~cm} \mathrm{X} 6 \mathrm{~cm})$ which were covered by a lid, incubated at $23{ }^{\circ} \mathrm{C}$ for 3-7 days and scored for soft rotting.

\subsubsection{Field experiments}

Bacterial inoculation of potato tubers and treatment with Degaclean were performed as described above (2.3 and 2.4) if not stated otherwise.

\subsubsection{Field experiments in Göttingen}

Potato tubers were artificially inoculated by a mixture of Ecc and Eca (3 strains of each subspecies) as described in 2.3, with the following specifications: potato tubers were at first wounded and then immersed in bacterial suspension (ca. $10^{8} \mathrm{cfu} / \mathrm{ml}$ ) for $3 \mathrm{~h}$. In 1999 inoculation was performed 2 days before planting on May, 10. In 2000 the variant E (see Table 1) was inoculated early (Jan. 15), the variants B and F late (May, 24). Treatment of potato tubers with Degaclean included three concentrations in 1999 (0.5, 1.0 and 2.0\%) and only one concentration (2.0\%) for the experiments of 2000 (in April), as described in 2.4.2.

In the second experiment (2000), two antibiotic-resistant mutants were used, Eca no. 2968 resistant against Rifampcin, and Ecc no. 2970 resistant against Rifampcin and Streptomycin (see 2.1). The potato tubers, cultivar Agria (obtained from Stöver Co.), were planted in April 2000.

The field experiments were performed in 1999 and 2000 on the experimental field of the Institute of Tropical and Subtropical Agronomy (“Am Wassergraben”) in Göttingen-Weende. The soil type was Typic Hapludalf, according to United States Dept. of Agriculture (USDA). Fertilizer was applied to the soil only in 2000 on June 9 in the form of $100 \mathrm{~kg} \mathrm{~N}$ per hectare of "Kalkammmonsalpeter" (= nitrate of lime and ammonia, i.e. mixture of ammonium nitrate and calcium carbonate), containing 27\% N. Potatoes were planted on May 12, 1999 and April 26, 2000 , by hand. The soil was dry and warm at the time of planting.

In 1999, the potato tubers (cultivar Bintje, obtained from Naturkost Elkershausen GmbH Göttingen) were only treated lately (just before planting in the field) with different concentrations of Degaclean, so that the following five variants resulted:

A - Inoculation with bacteria, no treatment with Degaclean 
B - Bacteria, and 0.5\% Degaclean

C - Bacteria, and 1.0\% Degaclean

D - Bacteria, and 2.0\% Degaclean

E - Without bacteria, no treatment with Degaclean (control)

Each variant consisted of 3 repetitions ( 3 rows of $15 \mathrm{~m}$ length with 50 potatoes per row), and the distance between the rows was $75 \mathrm{~cm}$ and $1.5 \mathrm{~m}$ between each variant (Fig. 1). Within the rows the potatoes were planted with spaces of $30 \mathrm{~cm}$. 


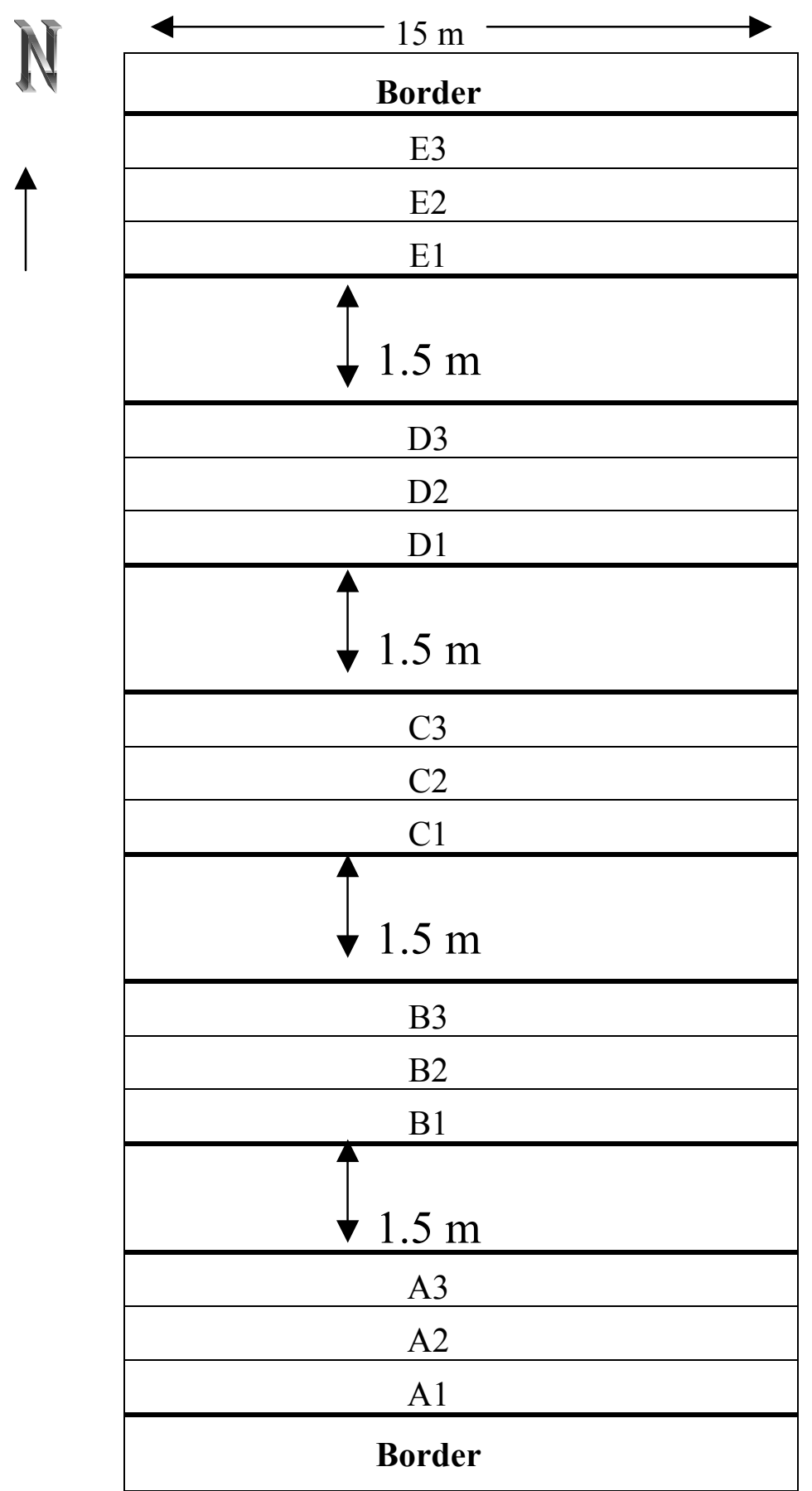

Fig. 1: Design of field experiments 1999 
In 2000 the field experiments consisted of 6 variants (Table 1)

Table 1: Variants of field experiments 2000

\begin{tabular}{|l|l|l|}
\hline Designation & Bacterial inoculation & Degaclean treatment \\
\hline A & - & - \\
\hline B & late, 24/04/2000 & - \\
\hline C & - & early, 15/01/2000 \\
\hline D & - & late, 25/04/2000 \\
\hline E & early, 14/01/2000 & early, 15/01/2000 \\
\hline F & late, 24/04/2000 & late, 25/04/2000 \\
\hline
\end{tabular}

In 2000, each variant consisted of 4 repetitions with 20 planted tubers, i.e in total 80 planted tubers per variant. The repetitions of the 6 variants were differently mixed on the 6 plots as shown in Fig. 2. Distances of potato plants within the rows and between rows were as described in field experiments 1999.

Weeds were removed by hoe, and dams were produced by mattock when the potato plants were $15 \mathrm{~cm}$ high. Chemical plant protection measures were performed only in 2000, that is, fungicide Shirlan (0.3 litre/ha, active substance 500g/l Fluazinam, conc. $0.13 \%)$ two months after planting, and fungicide Acrobat plus (1.5-2.0 kg/ha, active substance 9\% Dimethomorph $+60 \%$ Mancozeb, conc. 0.67\%) two weeks later, against Phytophthora infestans. In 1999, the potato plants were strongly affected by potato late blight (Phytophthora infestans) and had to be harvested early, on August 25 by hand. In 2000 the potatoes were harvested on September 7. 


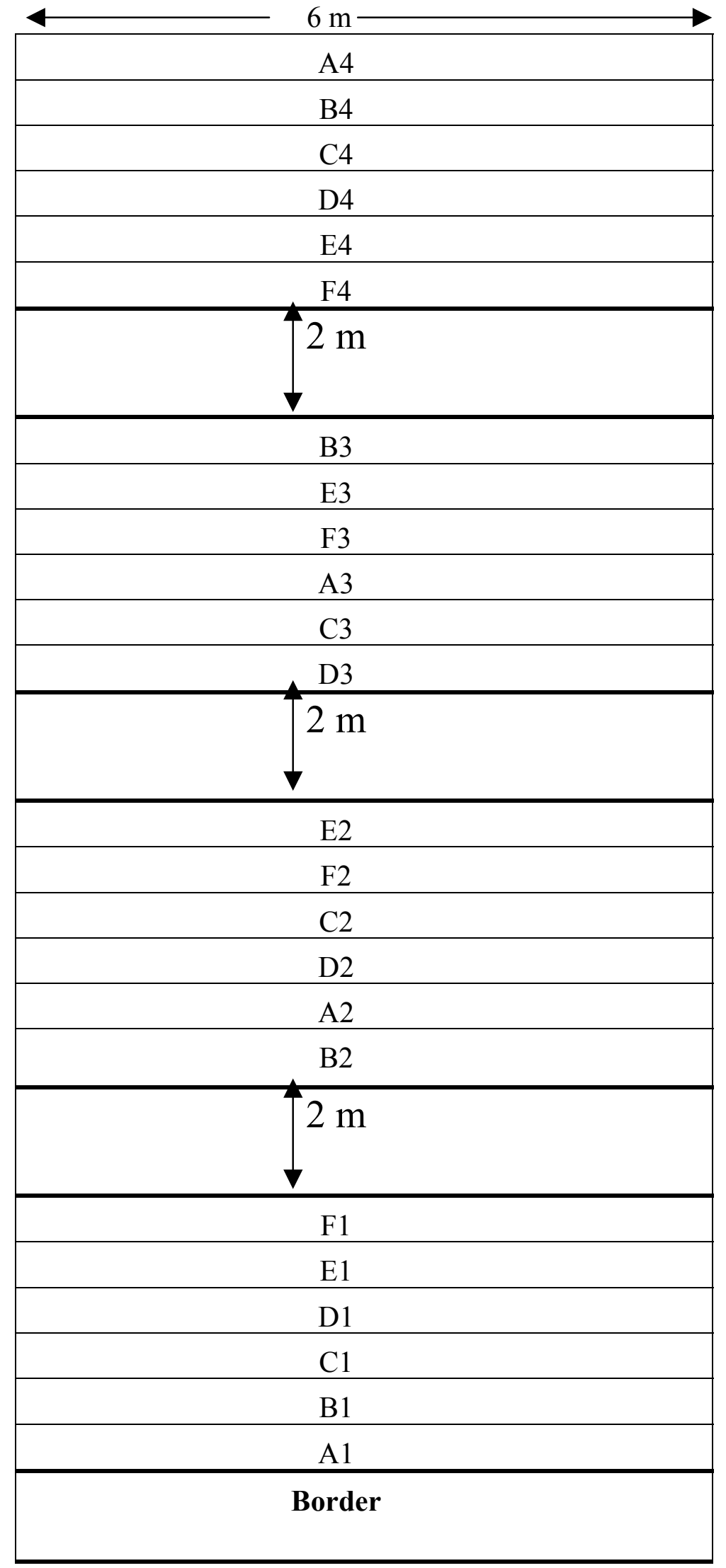

Fig. 2: Design of field experiments 2000 


\subsection{Evaluation at harvest}

The potatoes were harvested by hand and the tubers of each row (repetition) were collected in one sample. Rotted potato tubers were counted during harvesting and discarded. The healthy looking tubers were weighed and stored at $4{ }^{\circ} \mathrm{C}$ for $2-4$ weeks until evaluation of latent contamination.

The potatoes harvested from each plot were divided into six "groups" (repetitions), each of which was analysed separately for latent contamination. Evaluation of each separate "group" 2-4 weeks after harvest included recording the total weight of potato tubers, numbers of tubers with soft rot, and determination of latent contamination by Erwinia carotovora. For the latter purpose, each potato sample was immersed in $0.01 \mathrm{M} \mathrm{MgSO}_{4}$ for $3 \mathrm{~h}$ at $4{ }^{\circ} \mathrm{C}$ with occasional stirring every $30 \mathrm{~min}$ by hand. Finally $50 \mathrm{ml}$ were taken and analysed as described in Chapter I, 2.3.7.2.

\subsubsection{Field experiments by Stöver Co.}

The field experiments were performed in 1999 on a farm in Natenstedt near Twistringen to study the effect of Degaclean treatment on potato lots which had been found to be latently contaminated by Ecc in Göttingen (samples Se 4 and Se 5 listed in Chapter I, Table 15). In September 1998 or in April 1999 the tubers were strongly sprayed with 0.5\% Degaclean until the surfaces of the tubers became completely wet. Then the potato tubers were placed on filter paper at room temperature over night for superficial drying. The field experiments were performed and the potato tubers were planted as described earlier (Chapter I, 2.5). Each of the four variants consisted of 4 blocks, each with two rows of 24 potatoes totally, planted with a space of $37 \mathrm{~cm}$ (Fig. 3). Potatoes were planted on April 20, 1999 by hand. The four variants of the experiment were:

A - cv. Morene from Semlow (= Se 4), early treatment (in fall 1998) with 0.5\% Degaclean, B - cv. Morene from Semlow (= Se 4), no treatment with Degaclean,

$\mathrm{C}-\mathrm{cv}$. Morene from Semlow 33 ha (= Se 5), (strongly contaminated, degree 3), no Degaclean treatment,

D - cv. Morene from Semlow 33 ha (= Se 5), late treatment (in spring 1999) with 0.5\% Degaclean. 
The potatoes were harvested on September 20, 1999, by hand. Evaluation of these field experiments was performed as described in Chapter I, 2.5.

\begin{tabular}{|c|c|c|c|c|c|c|c|c|c|}
\hline Block 4 & A. & B. & C. & D. & & & & & \\
\hline Block 2 & A. & B. & C. & D. & A. & B. & C. & D & Block 3 \\
\hline & & & & & A. & B. & C. & D. & Block 1 \\
\hline
\end{tabular}

Fig. 3: Design of field experiments 1999 by Stöver Co.

\subsection{Characterization of lipopolysaccharides from Erwinia carotovora}

\subsubsection{Culture of bacteria and washing of cells}

Lipopolysaccharides were isolated from five strains as follows:

Eca GSPB no. 2962 from Japan which did not contain the LPS O-chain,

Eca GSPB no. 2967, highly virulent,

Ecc GSPB no. 436, very highly virulent,

Eca GSPB no. 2967 “a”, Degaclean-tolerant and less virulent than the parent strain,

Ecc GSPB no. 436 "a”, Degaclean-tolerant and less virulent than the parent strain.

At first, all the strains were grown on King's medium B (agar) as described in 2.3. Then, the bacteria were transferred into a liquid medium containing per Litre $10.0 \mathrm{~g}$ peptone from casein (Roth Co., no. 8986.2), 5.0 g glucose and 1.0 g casamino acids (Difco no. 0231-010). The medium was autoclaved at $121{ }^{\circ} \mathrm{C}$ for $15 \mathrm{~min}$, the bacterial cultures were shaken at $110 \mathrm{rpm}$ at $28{ }^{\circ} \mathrm{C}$ (Clim-O-shake, Firma Kühner, Basel, Switzerland). After $48 \mathrm{~h}$ of growth the bacteria were precipitated by centrifugation $\left(10000 \mathrm{X} \mathrm{g} / 4{ }^{\circ} \mathrm{C}\right)$ and washed three times with EDTAsaline, finally with water. The cells were frozen at $-20{ }^{\circ} \mathrm{C} / 24 \mathrm{~h}$ and lyophilized.

EDTA-saline

$10 \mathrm{~g} \mathrm{NaCl}$

$10 \mathrm{mM} \mathrm{Na} 2$-EDTA

\subsubsection{Extraction of LPS by the phenol-water method}

For extracting LPS, the lyophilized cells were suspended in water $(4 \mathrm{~g} / 100 \mathrm{ml})$ to give a slurry. The slurry was warmed to $70{ }^{\circ} \mathrm{C}$ and mixed with $90 \%$ warm phenol (1:1), the mixture was stirred for 20-30 min and cooled down in an ice-bath overnight. Phenol-water mixtures are monophasic at $70{ }^{\circ} \mathrm{C}$, but separate into two phases below that temperature. The two phases were separated by centrifugation $\left(10000 \mathrm{~g} / 4^{\circ} \mathrm{C}\right)$, then the upper watery phase was carefully siphoned off. It contains LPS, polysaccharides, RNA, and salts. The lower phase contains 
proteins, lipids, phospholipids, pigments, and DNA. The water phase was dialyzed against bidist. water for 4 days and finally lyophilized (Type BETA I, Christ Company).

\subsubsection{Polyacrylamide gel electrophoresis (PAGE)}

LPS was separated by gel electrophoresis in the Minigel-system (Biometra Company, Göttingen) with $10 \times 10 \mathrm{~cm}$ glass plates and $1 \mathrm{~mm}$ space. Electrophoresis was performed in sodium dodecyl sulfate-polyacrylamide gels (SDS-PAGE).

\subsubsection{Preparation of separation gel}

All the constituents, except ammoniumpersulphate (= APS) were put in a $10 \mathrm{ml}$, side-arm vacuum, conical flask. The flask was closed with a rubber stopper and the suspension was deaerated for 2 min under slight stirring, because oxygen may hinder polymerization. The glass plates of the apparatus were marked ca. $1 \mathrm{~cm}$ below the lower end of the combs, to ensure that the height of the stacking gel was at least 2 times more than the height of LPS samples to be poured. APS was mixed with the other components, stirred for $1 \mathrm{~min}$, and poured into the sandwich apparatus with a sterile glass pipette. About $1 \mathrm{~cm}$ bidest. water was layered over the gel to avoid contact of the gel with air. The gel polymerized within about $45 \mathrm{~min}$.

\subsubsection{Preparation of stacking gel}

After polymerization, the bd-water was poured off from the sandwich, and the stacking gel prepared as described in Table 2 was put into a $10 \mathrm{ml}$, side-arm vacuum, conical flask, and deaerated for 2 min under slight stirring. APS was mixed for $1 \mathrm{~min}$ and the combs were inserted into glass plates and the gel was gradually poured with a sterile glass pipette. All air bubbles were removed by moving the comb up and down. The stacking gel polymerized within $30 \mathrm{~min}$. 
Table 2: Receipts of separating and stacking polyacrylamide gels for LPS separation

\begin{tabular}{|l|c|c|}
\hline Ingredients & Separating gel (10\%) & Stacking gel (3\%) \\
\hline Acrylamide 30\%, 0.8\% Bisacrylamide & $2.0 \mathrm{ml}$ & $250 \mu \mathrm{l}$ \\
\hline Separating gel buffer & $2.0 \mathrm{ml}$ & \\
\hline Stacking gel buffer & & $500 \mu \mathrm{l}$ \\
\hline TEMED (10\%) & $100 \mu \mathrm{l}$ & $15 \mu 1$ \\
\hline APS (10\%) & $15 \mu 1$ & $1.750 \mu \mathrm{l}$ \\
\hline Bidistilled water & $3.0 \mathrm{ml}$ & $2.565 \mu 1$ \\
\hline Total & $7.115 \mathrm{ml}$ & \\
\hline
\end{tabular}

\subsubsection{Buffers, chemicals and reagents for electrophoretic studies}

Electrophoresis reagents of electrophoretic grade were purchased from

Roth (Karlsruhe), and Merck (Darmstadt)

1) Acrylamide stock solution

2) Separation gel $(3 X)$
$30 \%$ Acrylamide, $0.8 \%$ Bisacrylamide

$1 \mathrm{M}$ Tris

$0.3 \mathrm{~g}$ SDS

Add $100 \mathrm{ml}$ bd $\mathrm{H}_{2} \mathrm{O}$

pH 8.8 with $\mathrm{HCl}$

3) Stacking gel (3 X)

$$
\begin{aligned}
& 1 \mathrm{M} \text { Tris } \\
& 0.3 \mathrm{~g} \mathrm{SDS}
\end{aligned}
$$

Add $100 \mathrm{ml} \mathrm{bd} \mathrm{H}_{2} \mathrm{O}$

pH 6.8 with $\mathrm{HCl}$

4) TEMED (10\%)

$10 \%$ in bd $\mathrm{H}_{2} \mathrm{O}$

5) Ammoniumpersulphate APS (10\%)

$10 \%$ bd $\mathrm{H}_{2} \mathrm{O}$

6) Denaturation buffer

$0.0625 \mathrm{M}$ Tris

$2.0 \% \mathrm{SDS}$

$10 \%$ glycerol

$0.002 \%$ Bromphenolblue $\mathrm{pH} 6.8$ with $\mathrm{HCl}$ 
7) Electrode buffer (10X)

$$
\begin{array}{rl}
30.3 & \text { g Tris } \\
144 & \text { g glycine } \\
10 & \text { g SDS } \\
1000 & \mathrm{ml} \mathrm{bd} \mathrm{H}_{2} \mathrm{O}
\end{array}
$$

\subsubsection{Preparation of LPS samples}

Each sample was dissolved in a $1 \mathrm{ml}$ Eppendorf tube $(0.2-0.5 \mathrm{mg} / \mathrm{ml}$ water $)$ and mixed at a ratio of 1:1 with denaturation buffer. The mixture was incubated at $65{ }^{\circ} \mathrm{C} / 5 \mathrm{~min}$, after which DTE was added (final concentration $10 \%$ ), and the mixture was heated at $100{ }^{\circ} \mathrm{C}$ for 5 min (MÜLLER, 1998).

2.5.3.5 Detection of LPS in the gel using silver staining (HEUKESHOVEN and DERNICK, 1988)

A few gels were stained with silver by the following procedure:

1) Fixing ( $2 h)$ $31.25 \mathrm{ml}$ ethanol $(96 \%)$ $30.0 \%$ $10.00 \mathrm{ml}$ acetic acid (100\%) $10.0 \%$ add $100 \mathrm{ml} \mathrm{H}_{2} \mathrm{O}$ bidest

2) Incubation ( $2 \mathrm{~h})$

$$
\begin{array}{lc}
31.25 \mathrm{ml} \text { ethanol }(96 \%) & 30.0 \% \\
4.00 \mathrm{~g} \text { sodium acetate } & 0.5 \% \\
2.00 \mathrm{~g} \text { glutardialdehyde } & 0.5 \% \\
0.31 \mathrm{~g} \text { sodium thiosulphate } \mathrm{X} 5 \mathrm{H}_{2} \mathrm{O} & 0.2 \%
\end{array}
$$
add $100 \mathrm{ml} \mathrm{H}_{2} \mathrm{O}$ bidest

2) Washing with bd-water 3 times $10 \mathrm{~min}$

3) Staining (45 min) $\quad 0.1 \mathrm{~g} \mathrm{AgNO}_{3} \quad 0.1 \%$

$28.5 \mathrm{ml}$ formaldehyde $(35 \%) \quad 0.01 \%$ add $100 \mathrm{ml} \mathrm{bd} \mathrm{H}_{2} \mathrm{O}$

6) Developing (3-10 min) $2.5 \mathrm{~g} \mathrm{Na}_{2} \mathrm{CO}_{3} \quad 2.5 \%$ add $100 \mathrm{ml}$ bd $\mathrm{H}_{2} \mathrm{O}$

7) Stopping (5-10 min) $\quad 1.86$ g EDTA $\quad 0.5 \mathrm{M}$ add $100 \mathrm{ml} \mathrm{bd} \mathrm{H}_{2} \mathrm{O}$ 
8) Washing with bd-water 3 times $10 \mathrm{~min}$

The developing solution was shaken until the LPS bands appeared weakly, and the treatment was stopped when the bands were still clearly visible with a colourless background of the gel. The gel was then preserved in bd-water at $4{ }^{\circ} \mathrm{C}$. 


\section{RESULTS}

\subsection{Effect of Degaclean on reduction of potato soft rot in laboratory experiments}

\subsubsection{Development of a standardized method for inducing soft rot by artificial contamination of potato tubers}

Table 3 clearly shows that only a high bacterial concentration $\left(10^{8} \mathrm{cfu} / \mathrm{ml}\right)$ of Erwinia spp. caused partial rotting of wounded potato tubers. The low bacterial concentration $\left(10^{6} \mathrm{cfu} / \mathrm{ml}\right)$ never caused any infection. Tubers which were wounded after treating with a high bacterial concentration showed only very weak rotting. Tubers of cultivar Morene were more often infected than the other two cultivars (Bintje and Cilena). According to these results, in the following experiments the high bacterial concentration $\left(10^{8} \mathrm{cfu} / \mathrm{ml}\right)$ was applied, and potato tubers (cv. Morene) were wounded before soaking in bacterial suspension.

Table 3: Number of potato tubers with soft rot 5 days after soakage in bacterial suspension $(E c a$ und $E c c)$ before or after wounding

\begin{tabular}{|l|c|c|c|c|c|c|}
\hline & \multicolumn{2}{|c|}{$\begin{array}{c}\text { Wounding } \\
\text { before after } \\
\text { cultivar }\end{array}$} & \multicolumn{2}{c|}{$\begin{array}{c}\text { Wounding } \\
\text { before } \\
\text { treatment with } \\
\text { water }\end{array}$} & $\begin{array}{c}\text { Wounding } \\
10^{8} \mathrm{cfu} / \mathrm{ml}(E c a+E c c)\end{array}$ & $\begin{array}{c}\text { after } \\
\text { before } \\
10^{6} \mathrm{cfu} / \mathrm{ml}(E c a+E c c)\end{array}$ \\
\hline Bintje & $1 *)$ & 0 & 2 & 1 & 0 & 0 \\
\hline Cilena & 0 & 0 & 2 & 1 & 0 & 0 \\
\hline Morene & 0 & 0 & 4 & 1 & 0 & 0 \\
\hline
\end{tabular}

$*)=5$ potato tubers were used for each experiment

\subsubsection{Disease severity caused by Erwinia spp. after treatment of potato tubers with different concentrations of Degaclean}

Different concentrations of Degaclean reduced soft rotting of potato tubers only very weakly (Table 4) in comparison to the control which was not treated with Degaclean. Unexpectedly, the longest treatment with Degaclean $(60 \mathrm{~min})$ was less effective than treatment for 15 or 30 min. Most effective against soft rot by erwinias was a treatment with 2\% Degaclean for 30 min (Table 4). Because of these results potato tubers were treated with 2\% Degaclean for $30 \mathrm{~min}$ in the field experiments of 2000 . 
Table 4: Number of potato tubers with soft rot after treatment with different concentrations of Degaclean (Dgc.)

\begin{tabular}{|l|c|c|}
\hline Treatment & after 4 days & after 6 days \\
\hline Only bacteria, without Degaclean & $\left.4^{*}\right)$ & 5 \\
\hline Bacteria $+0.5 \%$ Dgc. $/ 15 \mathrm{~min}$ & 2 & 4 \\
\hline Bacteria $+0.5 \%$ Dgc. $/ 30 \mathrm{~min}$ & 2 & 4 \\
\hline Bacteria $+0.5 \%$ Dgc. $/ 60 \mathrm{~min}$ & 2 & 4 \\
\hline Bacteria $+1.0 \%$ Dgc. $/ 15 \mathrm{~min}$ & 2 & 4 \\
\hline Bacteria $+1.0 \%$ Dgc. $/ 30 \mathrm{~min}$ & 2 & 4 \\
\hline Bacteria $+1.0 \%$ Dgc. $/ 60 \mathrm{~min}$ & 3 & 4 \\
\hline Bacteria $+2.0 \%$ Dgc. $/ 15 \mathrm{~min}$ & 2 & 4 \\
\hline Bacteria $+2.0 \%$ Dgc. $/ 30 \mathrm{~min}$ & 1 & 3 \\
\hline Bacteria $+2.0 \%$ Dgc. $/ 60 \mathrm{~min}$ & 3 & 4 \\
\hline
\end{tabular}

$*)=5$ potato tubers were used per experiment

\subsection{Effect of Degaclean on reduction of blackleg and potato soft rot in field experiments}

\subsubsection{Field experiments 1999}

\subsubsection{Field experiments in Göttingen}

The results obtained (Table 5) show that even in the control (without bacterial inoculation, and without Degaclean treatment) latent contamination with Ecc and soft rotting occurred, but treatment of seed potatoes with bacteria decreased the number of germinating potatoes from $95.5 \%$ to $82.7 \%$, due to soft rot, and increased latent contamination of harvested potatoes from $3.1 \times 10^{3}$ to $4.6 \times 10^{5} \mathrm{cfu} / \mathrm{kg}$.

However, the late Degaclean treatment of seed potatoes (just before planting in the field) caused only a slight reduction of latent contamination at harvest from $4.6 \times 10^{5}$ to $3.5 \times 10^{4}$ $\mathrm{cfu} / \mathrm{kg}$ at the highest Degaclean concentration (2\%). Treatment with $0.5 \%$ Degaclean did not cause a significant reduction of latent contamination with Ecc.

Unexpectedly, Degaclean treatment significantly reduced the number of germinated potato tubers by $27.6 \%, 24.4 \%$ and $23.7 \%$ at the 3 concentrations, compared to potato tubers which were only treated with bacteria (17.3\%), and also the percentage of tubers with soft rot increased significantly after Degaclean treatment, especially at the low concentration of 0.5 and 
$1.0 \%$. Therefore, also the yields were reduced by all Degaclean treatments compared to the untreated variants. Potato plants with blackleg symptoms were not observed in the experimental field plots in 1999. All bacterial isolates which were obtained from these experiments were Ecc.

Table 5: Field experiments 1999: Effect of Degaclean on the number of latent contamination with Erwinia carotovora ssp. carotovora $(E c c)$

\begin{tabular}{|l|c|c|c|c|c|}
\hline \multicolumn{1}{|c|}{ Treatment } & $\begin{array}{c}\text { Number of non- } \\
\text { germinated } \\
\text { tubers in } \%\end{array}$ & \multicolumn{2}{|c|}{$\begin{array}{c}\text { Number of potato tubers } \\
\text { with soft rot at harvest } \\
\text { absolute per } 10 \mathrm{~kg}\end{array}$} & $\begin{array}{c}\text { Colony forming } \\
\text { units (cfu) per kg }\end{array}$ & $\begin{array}{c}\text { Yield in } \\
\mathrm{kg}\end{array}$ \\
\hline $\begin{array}{l}\text { Control, } \\
\text { without Bacteria, } \\
\text { without Degaclean }\end{array}$ & 4.4 & 31 & 2.9 & $3.1 \times 10^{3}$ & 107.4 \\
\hline $\begin{array}{l}\text { Bacteria, } \\
\text { without Degaclean }\end{array}$ & 17.3 & 27 & 2.8 & $4.6 \times 10^{5}$ & 95.3 \\
\hline $\begin{array}{l}\text { Bacteria, with } \\
0.5 \% \text { Degaclean }\end{array}$ & 27.6 & 32 & 4.1 & $2.5 \times 10^{5}$ & 78.9 \\
\hline $\begin{array}{l}\text { Bacteria, with } \\
1.0 \% \text { Degaclean }\end{array}$ & 24.4 & 35 & 4.0 & $2.9 \times 10^{4}$ & 87.2 \\
\hline $\begin{array}{l}\text { Bacteria, with } \\
2.0 \% \text { Degaclean }\end{array}$ & 23.7 & 28 & 3.2 & $3.5 \times 10^{4}$ & 86.4 \\
\hline
\end{tabular}

\subsubsection{Field experiments by Stöver Co.}

The field experiments of Stöver Co. aimed to compare Degaclean treatments of seed potatoes in fall and spring. Potato tubers from cultivar Morene, produced in Semlow, were either treated in fall (September 1998) or in spring (April 1999). In 1999 Degaclean treated and untreated potatoes from the same lot were grown in an experimental design with 4 repetitions (subchapter 2.5, page 18) on a farm in Natenstedt.

The results (Table 6) show that Degaclean-treatment in fall reduced the percentage of tubers with soft rot after harvest in the following year from 3.4 to $1.3 \%$. In contrast, treatment in spring did not reduce percentage of tubers with soft rot. Also, the appearance of blackleg symptoms in the field was reduced by Degaclean treatment in fall (BRUER, person. communication). The other parameters - yield and tuber sizes - were not affected by Degaclean-treatments. 
Table 6: Field experiments 1999 by Stöver Co. on the effects of Degaclean treatments in fall and spring on latent contamination with Erwinia spp.

\begin{tabular}{|c|c|c|c|c|}
\hline \multirow{2}{*}{$\begin{array}{l}\text { Soft rotting, } \\
\text { yield and tuber size }\end{array}$} & \multicolumn{2}{|c|}{ Treatment in fall } & \multicolumn{2}{|c|}{ Treatment in spring } \\
\hline & $\begin{array}{l}\text { untreated } \\
\text { control }\end{array}$ & Degaclean treated & untreated control & $\begin{array}{c}\text { Degaclean } \\
\text { treated }\end{array}$ \\
\hline Soft rotted tubers in \% & 3.4 & 1.3 & 5.4 & 5.4 \\
\hline Yield in $\mathrm{t} / \mathrm{ha}$ & \multirow[b]{2}{*}{1.81} & \multirow[b]{2}{*}{1.69} & \multirow[b]{2}{*}{2.06} & \multirow[b]{2}{*}{1.92} \\
\hline Fraction $35-40$ & & & & \\
\hline Fraction $40-50$ & 12.69 & 13.88 & 17.08 & 14.25 \\
\hline Fraction 50-55 & 11.38 & 11.50 & 11.58 & 11.83 \\
\hline Fraction 55-60 & 13.69 & 10.69 & 10.83 & 13.08 \\
\hline Fraction $60+$ & 17.13 & 18.44 & 11.83 & 12.50 \\
\hline Total & 56.7 & 56.2 & 53.4 & 53.6 \\
\hline $\begin{array}{l}\text { Tuber size } \\
\text { Number of tubers } / 10 \mathrm{~kg}\end{array}$ & \multirow[b]{2}{*}{52} & \multirow[b]{2}{*}{53} & \multirow[b]{2}{*}{54} & \multirow[b]{2}{*}{56} \\
\hline Fraction $50+$ & & & & \\
\hline Fraction $55+$ & 44 & 44 & 44 & 47 \\
\hline Fraction $60+$ & 36 & 37 & 36 & 37 \\
\hline
\end{tabular}

\subsubsection{Field experiments 2000}

For these experiments, antibiotic resistant mutants were generated from two strains, Eca 2968 and Ecc 436, by exposition to Rifampicin and Streptomycin. Before planting, potato tubers were inoculated with the antibiotic-resistant strains. By applying these strains it was possible to differentiate between erwinias originating from the tubers or from the soil and erwinias which had been applied on the potato tubers. Eca 2968 was resistant against 200 ppm Rifampicin and Ecc 436 was resistant against 200 ppm Rifampicin and 800 ppm Streptomycin.

The results obtained in these experiments (Table 7) show that the bacterial inoculation was also successful in this year, because the percentage of potato tubers with soft rot at harvest nearly doubled, and because the latent contamination with E. carotovora on the harvested potatoes increased tremendously (from $3.2 \times 10^{3}$ to $5.6 \times 10^{6} \mathrm{cfu} / \mathrm{kg}$ ).

Degaclean-treatment alone, without artificial inoculation of bacteria, reduced germination rate of potatoes badly, especially at the late application date shortly before planting. Since soft rotting of potatoes was simultaneously enhanced at harvest, Degaclean treatment resulted in yield reduction of $25-45 \%$. These negative effects were less at the earlier application date. 
Table 7: Effect of Degaclean on latent contamination with Erwinia spp. (Eca und Ecc) and on yield in field experiments 2000

\begin{tabular}{|c|c|c|c|c|c|}
\hline Treatment & $\begin{array}{l}\text { Number of } \\
\text { non- } \\
\text { germinated } \\
\text { tubers in \% }\end{array}$ & $\begin{array}{l}\text { Number } \\
\text { with sof } \\
\text { absolute }\end{array}$ & $\begin{array}{l}\text { tato tubers } \\
\text { at harvest } \\
\text { per } 10 \mathrm{~kg}\end{array}$ & $\begin{array}{l}\text { Colony forming } \\
\text { units (cfu) per } \\
\text { kg }\end{array}$ & Yield in $\mathrm{kg}$ \\
\hline $\begin{array}{l}\text { Control, } \\
\text { without Bacteria, } \\
\text { without Degaclean }\end{array}$ & 20 & 15 & 1.2 & $3.2 \times 10^{3}$ & 126.5 \\
\hline $\begin{array}{l}\text { Without Bacteria, } \\
\text { early Degaclean }\end{array}$ & 26.3 & 16 & 1.6 & $2.4 \times 10^{4}$ & 100.5 \\
\hline $\begin{array}{l}\text { Without Bacteria, } \\
\text { late Degaclean }\end{array}$ & 58.8 & 19 & 2.5 & $4.6 \times 10^{4}$ & 77.6 \\
\hline $\begin{array}{l}\text { Only Bacteria, } \\
\text { without Degaclean }\end{array}$ & 23.8 & 28 & 2.6 & $5.0 \times 10^{6}$ & 106.8 \\
\hline $\begin{array}{l}\text { Bacteria, } \\
\text { early Degaclean }\end{array}$ & 40 & 23 & 2.4 & $3.4 \times 10^{5}$ & 96.3 \\
\hline $\begin{array}{l}\text { Bacteria, } \\
\text { late Degaclean }\end{array}$ & 62.5 & 35 & 4.8 & $8.9 \times 10^{5}$ & 72.3 \\
\hline
\end{tabular}

A combination of bacterial inoculation and Degaclean treatment showed a small reduction of latent contamination of potato tubers at harvest. However, this positive effect was strongly exceeded by the negative effects, that is reduction of germination, higher percentage of soft rotted potatoes at harvest and lower yield. Thus, the yield was reduced by 10 and $32 \%$ by the early and late Degaclean treatment, respectively.

The results (Table 8) clearly show that the antibiotic-resistant Erwinia carotovora strains were isolated only from those experimental variants which had been inoculated by these bacteria (nos. 4, 5 and 6). Spontaneous mutants with resistance against Rifampicin and Streptomycin did never show up in variants no. 1-3, which had not been inoculated with antibiotic-resistant bacteria. Therefore, these experiments allowed to differentiate between latent contamination of harvested potatoes originating

a) from the seed potatoes before treatment or from the soil (natural contamination), or

b) from the artificially inoculated bacteria.

The experiments confirmed that late Degaclean-treatment (no. 6) resulted in higher latent contamination than early Degaclean-treatment (no. 5). But even the early Degaclean-treatment 
(no. 5) did not reduce latent contamination resulting from artificially inoculated bacteria (no. 4). Only the non-antibiotic-resistant erwinias were slightly reduced by Degaclean-treatment in nos. 5 and 6 compared to no. 4, as shown on the CVPM-semi-selective medium (see Chapter I, 3.2.3). However, these results were not observed in nos. 2 and 3 when compared to no. 1.

Because the artificially inoculated Erwinia carotovora subspecies differed in antibioticresistance (Eca was resistant against Rifampicin, while $E c c$ was resistant against Rifampicin and Streptomycin) the numbers of Eca 2968 isolates (column 5) could be calculated by subtracting the values in column 6 from those in column 4.

On the field, blackleg symptoms were observed in three variants two months after planting, that is in each of the following variants two plants with blackleg symptoms occurred: early and late Degaclean treatment without bacterial inoculation and inoculation of bacteria only.

Table 8: Detection and isolation of soft rotting Erwinia spp. on potato tubers from field experiments in 2000 on three different media

\begin{tabular}{|l|l|l|c|c|c|}
\hline \multirow{2}{*}{ No. } & Treatment & \multicolumn{3}{|c|}{ cfu/kg potato tubers } \\
\cline { 2 - 6 } & & CVPM $^{\mathbf{1}}$ & KB with Rifampicin & \multicolumn{2}{|c|}{$\begin{array}{l}\text { KB with Rifampicin } \\
\text { and Streptomycin } \\
\text { Ecc 2970 }\end{array}$} \\
\hline 1 & $\begin{array}{l}\text { Control, } \\
\text { without Bacteria, } \\
\text { without Degaclean }\end{array}$ & $3.8 \times 10^{3}$ & Eca 2968+Ecc 2970 & Eca 2968 & 0 \\
\hline 2 & $\begin{array}{l}\text { Without Bacteria, } \\
\text { early Degaclean }\end{array}$ & $5.9 \times 10^{4}$ & 0 & 0 & 0 \\
\hline 3 & $\begin{array}{l}\text { Without Bacteria, } \\
\text { late Degaclean }\end{array}$ & $5.7 \times 10^{4}$ & 0 & 0 & 0 \\
\hline 4 & $\begin{array}{l}\text { Only Bacteria, } \\
\text { without Degaclean }\end{array}$ & $1.1 \times 10^{5}$ & $3.6 \times 10^{3}$ & $2.2 \times 10^{3}$ & $1.4 \times 10^{3}$ \\
\hline 5 & $\begin{array}{l}\text { Bacteria, } \\
\text { early Degaclean }\end{array}$ & $5.2 \times 10^{4}$ & $4.4 \times 10^{3}$ & $1.2 \times 10^{3}$ & $3.2 \times 10^{3}$ \\
\hline 6 & $\begin{array}{l}\text { Bacteria, } \\
\text { late Degaclean }\end{array}$ & $9.5 \times 10^{4}$ & $1.1 \times 10^{4}$ & $4.8 \times 10^{3}$ & $6.3 \times 10^{3}$ \\
\hline
\end{tabular}

1) $\mathrm{CVPM}=$ crystal violet pectate medium modified (see Chapter I, 3.2.3)

2) $0=$ no growth 


\subsection{Generation of Degaclean tolerant strains of Erwinia carotovora ssp. carotovora and ssp. atroseptica}

\subsubsection{Sensitivity against different concentrations of Degaclean}

Cultures of the strains Eca 2967 and Ecc 436 were consecutively exposed to increasing concentrations of Degaclean (see II, subchapter 2.2) to investigate whether the bacteria could develop tolerance against certain Degaclean concentrations. As shown in Table 9, after exposure to $1.2 \%$ Degaclean for $24 \mathrm{~h} 2.5-3.1 \times 10^{4} \mathrm{cfu} / \mathrm{ml}$ were detected in the nutrient medium, indicating that these bacterial cells were tolerant against $1.2 \%$ Degaclean. Thus, from both strains mutants could be selected which were tolerant against 1.2\% Degaclean.

However, 1.5\% Degaclean completely killed all bacterial cells. From the growth medium with $1.2 \%$ Degaclean one isolate each of $E c a$ and Ecc (Degaclean-tolerant isolates) was selected and stored in sterile water to further investigate other bacterial features, such as virulence and chemical structure of LPS. These strains were also included into the lyophilized bacterial collection (GSPB nos. 447 and 448).

Table 9: Bacterial concentration of two E. carotovora strains in King's medium B after exposure to different concentrations of Degaclean after growth for $24 \mathrm{~h}$

\begin{tabular}{|l|c|c|}
\hline Treatment & Eca $2967 \mathrm{cfu} / \mathrm{ml}$ & Ecc $436 \mathrm{cfu} / \mathrm{ml}$ \\
\hline $0 \%$ control & $6.2 \times 10^{7}$ & $9.8 \times 10^{7}$ \\
\hline $0.3 \%$ Degaclean & $3.2 \times 10^{7}$ & $5.2 \times 10^{7}$ \\
\hline $0.6 \%$ Degaclean & $8.2 \times 10^{6}$ & $6.1 \times 10^{6}$ \\
\hline $0.9 \%$ Degaclean & $6.2 \times 10^{5}$ & $1.1 \times 10^{5}$ \\
\hline $1.2 \%$ Degaclean & $2.5 \times 10^{4}$ & $3.1 \times 10^{4}$ \\
\hline $1.5 \%$ Degaclean & 0 & 0 \\
\hline
\end{tabular}

\subsubsection{Virulence}

The virulence of the generated Degaclean-tolerant strains was tested by inoculation of potato tubers slices (see I, subchapter 2.2.4). Results (Table 10) show that the mutant strains from both subspecies were less virulent than the corresponding wild parent strains in all experimental variants. It was also observed that the $E c c$ strains were more virulent than the $E c a$ strains in these tests. 
Table 10: : Determination of virulence of four strains of Erwinia spp. on potato tuber slices by determining diameter of rotted tissue in $\mathrm{cm}$. Designation "a" indicates Degaclean-tolerant strains

\begin{tabular}{|l|c|c|c|c|c|}
\hline & \multicolumn{5}{|c|}{ Bacterial concentration } \\
Strain & $1.2 \times 10^{8}$ & $1.2 \times 10^{7}$ & $1.2 \times 10^{6}$ & $1.2 \times 10^{4}$ & $1.2 \times 10^{3}$ \\
\hline Eca 2967 & $2.0 *)$ & 1.3 & 1.0 & 0 & 0 \\
\hline Eca 2967 "a" & 1.3 & 1.0 & 0 & 0 & 0 \\
\hline Ecc 436 & 3.0 & 2.3 & 1.5 & 0 & 0 \\
\hline Ecc 436 "a" & 1.7 & 1.3 & 0 & 0 & 0 \\
\hline
\end{tabular}

*) $=$ the rotting of slices (diameter of soft rot tissue in $\mathrm{cm}$ ) was measured after $48 \mathrm{~h}$

\subsubsection{Effect of Degaclean-tolerance on LPS}

Lipopolysaccharides from E. carotovora strains were separated by SDS-PAGE electrophoresis (Fig. 4). The stained gels revealed a characteristic ladder-like pattern for the 4 bacterial strains from GSPB. However, a distinct difference between the wild strains (nos. 2967 and 436) and the corresponding Degaclean-tolerant strains (nos. 2967 "a" and 436 "a") was not observed. The LPS from the Japanese strain Eca 2962 did not show the typical ladder-like pattern. Obviously, the LPS of Eca 2962 did not contain an O-chain, indicating a "rough" LPS.

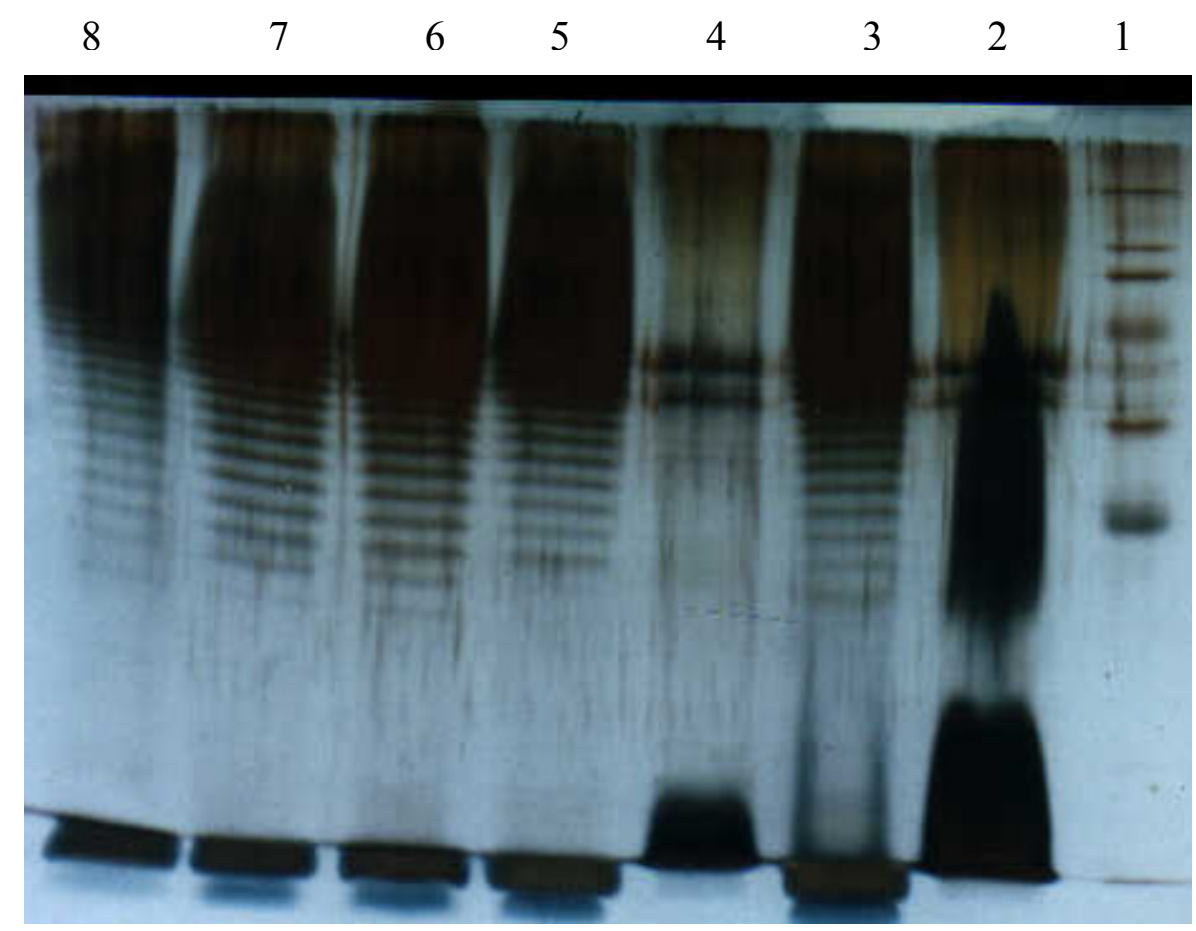

Fig. 4: SDS-PAGE of lipopolysaccharides from 5 Erwinia carotovora strains. The samples were run on a 10\% acrylamide gel. Lanes: (1) Marker VIII, (2) Eca 2962, (3) Ecc 436, (4) Eca 2962, (5) Ecc 436 “a” (Degaclean-tolerant), (6) Eca 2967, (7) Eca 2967 "a” (Degacleantolerant), and (8) Ecc 436. Into each slot $10 \mu \mathrm{LPS}$-solution (containing 0.2-0.5 mg/ml water) were added. Only no. 2 contained $15 \mu 1$. 
The disease control of blackleg (Erwinia carotovora ssp. atroseptica) relies primarily on the production of healthy potato tuber seeds (PEROMBELON and HYMAN, 1992). To guarantee the health status of the seed stock, seed classification was traditionally based on a visual crop inspection for blackleg affected plants. Blackleg incidence in the field is related to tuber contamination, but can be affected by environmental and cultural factors (DE BOER et al., 1996).

Resistant varieties may be part of the solution. The culture of resistant potatoes cultivars could be useful for the control of soft rot Erwinia species, as no chemical means of control are available (LAPWOOD \& GANS, 1984; DÖPKE and RUDOLPH, 1990; STEFANI et al., 1990). Resistance to soft rot is common within the gene resources of Solanum tuberosum (CIAMPI-PANNO and ANDRADE-SOTO, 1984) and of related species (VAN SOEST, 1983). One resistance source correlated with a high level of pectin methylation, increasing resistance of cell walls to enzyme degradation, was identified from the non-tuber-bearing Solanum brevidens and transferred to Solanum tuberosum spp. (PEROMBELON \& SALMOND, 1995). However, resistance as well as other measures, such as seed certification, good crop husbandry and farm hygiene may help, but are not totally effective (BOURGEOIS and PRIOU, 1995). Therefore, the aim of this study was to investigate the effect of Degaclean-treatment on reduction of blackleg and / or soft rot of potatoes in storage as well as in the field. The advantage of Degaclean is that no noxious residues are released, because the active substances are very soon transformed into environmentally friendly compounds. Thus, the amount of $\mathrm{H}_{2} \mathrm{O}_{2}$ was $0.04 \%$ after application, but 15 min later the amount was reduced to $0.006 \%$, and after 2 - $4 \mathrm{~h}$ the active oxygen was no longer detectable (STEINKAMP, 1998).

\subsection{Comparison of methods for artificial inoculation}

In order to study the effect of Degaclean on potato soft rot and potato blackleg a reliable method for inoculating potato tubers mimicking natural conditions was needed. The results of these studies clearly show that only a high bacterial concentration $\left(10^{8} \mathrm{cfu} / \mathrm{ml}\right)$ of Erwinia spp. caused partial rotting of wounded potato tubers. The low bacterial concentration $\left(10^{6} \mathrm{cfu} / \mathrm{ml}\right)$ never caused any infection. Tubers which were wounded after treating with a high bacterial concentration showed only very weak rotting. Tubers of cultivar Morene were more often infected than the other two cultivars (Bintje and Cilene).

Our experiments confirmed results from other workers that many soft rotting bacteria 'may be present superficially in lenticels and suberized wounds in most of the seed stocks, and that 
under favourable conditions the bacteria are transmitted to the lenticels of progeny potatoes during harvesting and grading where wounds caused by mechanical damage can become infected by bacteria (PEROMBELON and KELMAN, 1980). Also TOTH et al. (1996) reported that several sources for contamination can lead to blackleg infection, such as rain, insects, irrigation water via the soil from rotting mother tubers, irrigation water, or from contamination of wound sites caused during harvesting. Extensive contamination also occurs during harvesting and grading when erwinias from occasional rotting tubers spread to fresh wounds, once the bacteria can persist until planting time (PEROMBELON and HYMAN, 1995).

\subsection{Effect of Degaclean on reduction of soft rot in laboratory experiments}

NIEPOLD (1999b) reported that in laboratory experiments only 100 to $200 \mathrm{ppm}$ of the Clarmarin/KH10 mixture with 1-2 min of incubation time were required to absolutely destroy a cell suspension of $10^{9}$ cells per $\mathrm{ml}$ of two quarantine bacteria (Ralstonia solanacearum and Clavibacter michiganensis ssp. sepedonicus), suspended in an aqueous solution (Ringer). These results indicated that the possibility now exsists to efficiently interrupt the infection cycle of quarantine bacteria. For killing all bacterial cells of the same concentrations $\left(10^{9}\right.$ cells per $\mathrm{ml}$ ) of Ralstonia solanacearum in sewage water, $1000 \mathrm{ppm}$ of the Clarmarin/KH10 mixture and incubation for $20 \mathrm{~min}$ were necessary (NIEPOLD, 1999b). The tenfold higher concentration of Clarmarin/KH10 and the longer incubation time were explained by the presence of other organic material in the sewage water, reacting also with the peroxides.

Also, STEINKAMP (1998) reported that the bacterial concentration of Erwinia carotovora was reduced from ca. $1 \times 10^{8}$ to ca. $1 \times 10^{4}$ cells per potato tuber after washing with $5 \%$ Degaclean ${ }^{\circledR} 97$ for $0.5 \mathrm{~min}$. When the potato tubers were washed with the same concentration for $15 \mathrm{~min}$ they were completely free from bacteria. Our results revealed that the different concentrations of Degaclean reduced actual soft rotting of potato tubers only very weak, but most effective was a treatment with $2 \%$ Degaclean for $30 \mathrm{~min}$. Unexpectedly, the longest treatment with Degaclean (60 min) was less effective than treatment for 15 or $30 \mathrm{~min}$.

Thus, the results obtained in this study showed that a slight reduction of potato rotting by Degaclean $(2 \%)$ can be achieved. It appears necessary that experiments are repeated several times with different concentrations of Degaclean to find out whether a Degaclean-treatment may be suitable to protect food potatoes against soft rotting during long storage periods. 


\subsection{Field experiments}

\subsubsection{Effect of Degaclean on sprouting of potato tubers}

STEINKAMP (1998) studied germination and sprouting of potato tubers cvs. Gloria and Cilene in storage within 4 weeks after washing with Degaclean ${ }^{\circledR} 97$. In these experiments, a negative effect of Degaclean was never observed. In contrast, it appeared as if the Degacleantreated potatoes had a slightly higher number of sprouts than the untreated control.

On the other hand, our field experiments revealed that the newer preparation Degaclean ${ }^{\circledR} 150$ significantly reduced the number of germinating potatoes by $24-28 \%$ at all Degaclean concentrations used. The reason for these conflicting results is still unknown. However, we observed that all the non-germinating potato tubers in the field showed a strong soft rot. Therefore, it was concluded that treatment with Degaclean ${ }^{\circledR} 150$ may have somehow damaged the potato tubers at the outside or inhibited the natural resistant or warding off reactions, so that these tubers were infected by always present bacterial populations of Erwinia carotovora. However, the observations of STEINKAMP (1998) by scanning microscopy did not reveal any visible damage of the potato peel after Degaclean-treatment. Since also these results of STEINKAMP (1998) differed from our findings, it should be studied whether the different Degaclean-preparations (Degaclean ${ }^{\circledR} 97$ tested by STEINKAMP and Degaclean ${ }^{\circledR} 150$ in our experiments) may explain these conflicting results. According to the producer (Degussa Co., Mrs. G. Mitschke) Degaclean 150 contains 15\% active substance, Degaclean 97 only 5\% active substance. In addition, the combination of stabilizing compounds differs in the two preparations.

\subsubsection{Effect of Degaclean on soft rotting of potato tubers}

The results obtained by Stöver Co. showed that Degaclean-treatment in fall reduced the percentage of tubers with soft rot after harvest in the following year from 3.4 to $1.3 \%$. In contrast, treatment in spring did not reduce percentage of tubers with soft rot. Our results clearly showed that the percentage of tubers with soft rot increased significantly after Degaclean treatment, especially at the low concentration of 0.5 and $1.0 \%$. However, the earliest Degaclean-treatments in our experiments were performed rather late, on January 15 , that is 4 months after harvest.

\subsubsection{Effect of Degaclean-treatment on potato yields}

The results obtained by Stöver Co. show that the other parameters - yield and tuber sizes were not affected by Degaclean-treatments. Our results revealed that in the year of 1999 the 
yields were reduced by all Degaclean treatments compared to the untreated variants. Thus, the yield was reduced by 10 and $32 \%$ by the early and late Degaclean treatment, respectively, indicating that the negative effects were less at the earlier application date.

\subsubsection{Effect of Degaclean on reduction of latent contamination of potatoes}

The results obtained in this study showed that the late Degaclean treatment of seed potatoes (just before planting in the field) caused only a slight reduction of latent contamination at harvest from $4.6 \times 10^{5}$ to $3.5 \times 10^{4} \mathrm{cfu} / \mathrm{kg}$ at the highest Degaclean concentration (2\%). Other concentrations had no effects. However, this positive effect was strongly exceeded by the negative effects, that is reduction of germination, higher percentage of soft rotted potatoes at harvest and lower yield. Much more positive effects of Degaclean ${ }^{\circledR} 97$ were reported by STEINKAMP (1998). Treatment of potatoes with 5\% Degaclean for 0.5 min reduced the latent contamination from $10^{8}$ to $10^{4} \mathrm{cfu} /$ tuber, and treatment with the same concentration for $15 \mathrm{~min}$ destroyed nearly all latently occurring Erwinia carotovora cells on potato tuber surfaces.

\subsubsection{Differentiation of Eca and $E c c$}

Our results showed that all bacterial isolates obtained in the 1999 field experiments were Ecc. In contrast, in the 2000 field experiments the bacterial isolates were approximately $50 \%$ Eca and $50 \%$ Ecc. A possible explaination for these conflicting results is that the new semiselective medium favours the growth of $E c c$ more than that of $E c a$, so that the small growth cavities of Eca may be inhibited by the saprophytic bacteria in the Petri plates. Our studies with pure bacterial cultures indicated that cavities of Ecc strains on the semi-selective medium were broader and deeper than those of Eca. Therefore, the possibility can not be excluded that contaminations by $E c a$ were overlooked in some of the potato samples because the cavities produced by Eca were much smaller than those by Ecc. On the other hand, in the 2000 field experiments with antibiotic resistant strains of Ecc and Eca, the survey of the potatoes harvested clearly showed that also Eca contamination was detected on King's medium B which was supplemented with rifampicin. Thus both, of Eca and Ecc, were found together on the potatoes.

Another possibility for the exclusive isolation of Ecc in 1999 may be that subcultures were only carried out from the bigger growth cavities on the CVPM medium and, therefore, Eca strains were not isolated from latently contaminated potatoes in 1999. Also, the results obtained in the 2000 field experiments showed that Eca and Ecc were isolated only from the potatoes which were inoculated by the antibiotic-resistant strains but not from non-inoculated 
potatoes (Table 8). Further extensive experiments have to find out whether the sensitivity of the CVPM medium to detect contamination with Ecc and Eca under natural conditions differs because the semi-selective medium may be suited better for Ecc than Eca due to a faster growth after the standard incubation period.

\subsection{Effect of Degaclean-tolerance on LPS structure}

The bactericidal effect of Degaclean is due to the relase of hydrogen peroxide. Especially the extrem reactive radical $\mathrm{O}^{*}---$ oxidizes very fast diverse cell compounds, such as unsaturated fatty acids (ROTILIO et al., 1973). Therefore, the possibility existed that Degaclean-tolerant strains pocessed altered lipopolysaccharides (LPS). Our results obtained in these studies did not indicate that the LPS of Degaclean-tolerant strains differed in the O chain structure when compared to the wild parent strains which were not treated. However, the lipid LPS component (lipid A) of Degaclean-tolerant strains was not analysed in our studies.

GREGORY and FRIDOVICH (1973) reported that superoxide dismutase is an important component of the defences of Escherichia coli against the toxicity of oxygen. Due to these results it can be expected that the Degaclean-tolerant strains may have a higher superoxide dismutase. This aspect has to be investigated by further studies on the levels of superoxide dismutase and its association with a greater tolerance of hyberbaric oxygen. Results obtained in these studies also showed that the mutant strains from both subspecies were less virulent than the corresponding wild parent strains in all experimental variants. Obviously, physiological or biochemical alterations responsible for Degaclean-tolerance led to a decreased virulence compared to the wild parent strains. 


\section{SUMMARY}

1- In laboratory experiments treatment of potato tubers with $0.5,1.0$ and $2.0 \%$ Degaclean ${ }^{\circledR}$ 150 weakly reduced soft rotting of potato tubers by Erwinia carotovora ssp. carotovora and atroseptica. Most effective was a treatment with 2\% Degaclean for $30 \mathrm{~min}$.

2- In field experiments treatment of seed potatoes with Degaclean caused a slight reduction of latent contamination of harvested potato tubers. However, Degaclean-treatment also reduced the germination rate of seed potatoes and increased percentage of tubers with soft rot, so that yields after treatment of seed potatoes were reduced.

3- These negative effects of Degaclean were strongest at a late application, for instance just before planting of seed potatoes. The optimal application date appears to be immediately after harvest of potatoes.

4- Future experiments have to reveal whether the positive effects of Degaclean-treatment at the early application exceed the negative effects on germination.

5- Strains of Eca and Ecc developed tolerance against Degaclean concentrations up to 1.2\%. However, the virulence of the tolerant "mutants" was lower than that of the wild strains.

6- The mechanism of Degaclean-tolerance is unknown. LPS appeared to be unaltered in the tolerant strains. 


\section{CHAPTER III}

\section{Chemical structure of the LPS-O-chain of Erwinia carotovora and its}

\section{possible role in virulence}

\section{INTRODUCTION}

All Gram-negative bacteria contain lipopolysaccharides (LPS) which are complex molecules containing polysaccharides, fatty acids and phosphates. The LPS molecule consists of three components: lipid A and the sugar moieties, core region and the so-called O-specific chain (RIETSCHEL and BRADE, 1992; KNIREL and ZDOROVENKO, 1997). Thus, LPS forms an asymmetric bilayer with phospholipids such as phosphatidylethanolamine on the bacterial cell surface (SHUKLA et al., 1980). By means of their lipid moiety the LPS are anchored in the outer bacterial membrane and form a major constituent of it.

The outer membrane of Gram-negative bacteria acts as an impermeable barrier against hydrophobic molecules, owing to its highly ordered molecular assembly, and protects the cell from or adapts it to environmental changes (COSTERTON et al., 1974; NIKAIDO and VAARA, 1985). As LPS contains polysaccharide, fatty acids and phosphates, the formation of vesicle-like particles in water can be expected for this type of amphiphile (HANNECART et al., 1973). Therefore, LPS is also secreted into the surrounding medium (RAMM, 1993; SCHRÖDER, 2000; SCHRÖDER et al., 2001). According to FUKUOKA et al. (1989) also Erwinia carotovora produced extracellular LPS.

The LPS antigens have been especially valuable in serological classification of enterobacteria (EDWARDS and EWING, 1972), and the serological specificities of LPS have been useful criteria for differentiating several species into serological groups. Also for Erwinia carotovora, attempts have been made to characterize serological specificity, fatty acid composition and the structure of the core saccharide of Erwinia carotovora using cellular LPS extracted from the cells (DE BOER et al., 1979; RAY et al., 1987). DE BOER et al. (1985) determined the serological specificity and sugar composition of LPS purified from 16 Erwinia carotovora strains and differentiated six serogroups. The electrophoretic behaviour of lipopolysaccharides purified from Erwinia carotovora subsp. atroseptica serogroups I, XVIII, XX and XXII was investigated to see if there was a correlation between electrophoretic behavior and serogroup (MURRAY et al., 1990). Of 20 strains received as serogroup I, 18 gave a pattern identical to an authentic serogroup I strain. The two strains which did not give the same pattern were shown by immunological tests not to be serogroup I. Five atroseptica strains of serogroup 
XXII gave a distinict pattern characteristic of the serogroup while atroseptica strains of serogroup XVIII (four strains) and XX (five strains) gave patterns that could not be distinguished from each other.

Also for other plant pathogenic bacteria, especially the Pseudomonas syringae-group (OVOD et al. 1997; 1999) and the Xanthomonas campestris-group (SENCHENKOVA et al. 1999) the LPS may serve as a taxonomic character for differentiation between pathovars. In all these cases, the LPS-specificity is due to the structure of the O-specific chain. It is long known that changes in LPS O-chain structure can cause changes in electrophoretic mobility of lipopolysaccharides in SDS-PAGE system (HITCHCOCK et al., 1986).

Besides their usefulness for serological diagnosis LPS may also play an important role in pathogenesis. In human pathogenic bacteria, LPS exhibit many biological functions: mitogen activity, endotoxicity, Schwartzman reaction and others (LÜDERITZ et al., 1982; ALEXANDER and RIETSCHEL, 1999). The pathogenicity of soft rot causing bacteria of the genus Erwinia has been studied extensively in regard to excretion of pectinolytic enzymes (BARRAS et al., 1994; COLLMER and BAUER, 1994; PEROMBELON and SALMOND, 1995), whereas not much is known on the role of LPS. The association of LPS with certain enzymes has been studied to elucidate the interactions between lipopolysaccharide from Salmonella typhimurium and release of alkaline phosphatase (LINDSAY et al., 1973), or the interactions of the lipopolysaccharide from Escherichia coli with lysozyme (OHNO and MORRISON, 1989). The role of LPS in bacterial-plant interactions is likely to be complex. As well as providing an indispensable barrier for the bacteria against toxic plant compounds (NIKAIDO and VAARA, 1985), the interaction of LPS with the plant cell may promote pathogenesis or symbiosis (GROLMS, 1996; LAUX, 1998; VENKATESH and RUDOLPH, 2001; DAZZO et al., 1991) and/or may trigger defense-related responses (MÜLLER, 1998; MÜLLER et al., 1998).

Thus, GROLMS and RUDOLPH (1997) found that when the components originated from a compatible mixture, i.e. pectins from leaves susceptible to the Pseudomonas syringae pathovars, interactions were characterized by increased viscosity and yield stress. Also, LAUX et al. (1998) reported that the LPS from a smooth (s)-strain and a less virulent (r)-mutant of Pseudomonas syringae $p v$. phseolicola reacted differently with pectins from a susceptible bush bean. It was concluded from these studies that a synergistic interaction between plant pectins and bacterial LPS forms a gel-like matrix in the intercellular space and thus allows high multiplication of the bacteria in the susceptible reaction (RUDOLPH, 1999). Although several bacterial virulence factors play a role in host/pathogen-interaction during development of leaf 
spots, neither bacterial toxins nor avirulent genes can until now explain the narrow hostspecificity of the Pseudomonas syringae pathovars on the species level, and it was suggested that interactions between bacterial LPS and host polymers may be decisive (RUDOLPH, 1998). Also, in soft rot diseases caused by Ecc or Ech a low virulence of the bacterial pathogens has been correlated with defects in the LPS, especially the O-chain (SCHOONEJANS et al., 1987; PIRHONEN et al., 1991; TOTH et al., 1999).

The chemical structure of LPS of Erwinia carotovora has been analysed regarding the core region (SANDULACHE and PREHM, 1985; FUKUOKA et al., 1997) and the lipid A (FUKUOKA et al., 1992). However, the chemical structure of the LPS-O-chain of Eca and Ecc has never been elucidated. Therefore, structural studies of Erwinia LPS appear to be important for better understanding its role in the bacterium/plant-interaction and in the specific phase behaviour of LPS-containing membranes (FUKUOKA et al., 1990; FUKUOKA et al., 1995).

In this study lipopolysaccharides (LPS) from five Erwinia strains of the GSPB collection have been investigated extensively for differentiation between Erwinia carotovora subsp. atroseptica 9262 without LPS-O-chain (FUKUOKA, 1995; 1997) and other Erwinia subspecies. The purpose of this study was to investigate the chemical structure of the LPS-Oside chain and its role in virulence. 


\section{MATERIAL AND METHODS}

\subsection{LPS characterization}

\subsubsection{Extraction and purification}

The method was described in chapter II (see subchapter 2.5.2).

\subsubsection{SDS polyacrylamide gel electrophoresis (PAGE)}

The separation and stacking gels were prepared as described in chapter II (2.5.3.1 and 2.5.3.2). Combs were pulled out carefully, wells were rinsed with electrophoretic buffer $1 \mathrm{x}$ half strength of stock solution (see chapter II, 2.5.3.3). The samples were prepared as described in chapter II (2.5.3.4). The samples were applied with $10 \mu$ LPS-solution in each slot. Only slot no. 2 contained $15 \mu$ LPS-solution. The LPS-solutions contained 0.2-0.5 mg/ml water.

The samples were separated first at $10 \mathrm{~mA}$ for $15 \mathrm{~min}$ and then changed to $20 \mathrm{~mA}$ for $1-1.5$ hours. Electrophoresis was stopped when the front of the LPS samples had travelled to about 1 $\mathrm{cm}$ above the lower end of the gel. The gel was removed and put into a big Petri dish, then treated with other chemicals as described in chapter II, 2.5.3.3.

\subsection{Structure analysis of the LPS-O-chain}

These experiments were carried out in the laboratory of Dr. KNIREL (Zelinsky Institute of Organic Chemistry, Russian Academy of Sciences, Leninsky Prospekt 47, Moscow 117913, Russian Federation). The O-chain polysaccharide (OPS) was prepared by degradation of the LPS sample with aq. $2 \% \mathrm{AcOH}$ for $1.5 \mathrm{~h}$ at $100{ }^{\circ} \mathrm{C}$ followed by GPC on a columm $(70 \times 2.6$ $\mathrm{cm}$ ) of Sephadex G-50 using $0.05 \mathrm{M}$ pyridinium acetate buffer ( $\mathrm{pH} 4.5)$ as eluent and monitoring with a Knauer differential refractometer.

Sugar analysis: The OPS (0.5 mg) was hydrolyzed with $2 \mathrm{M} \mathrm{CF}_{3} \mathrm{CO}_{2} \mathrm{H}\left(120{ }^{\circ} \mathrm{C}, 2 \mathrm{~h}\right)$; monosaccharides were identified by GLC as their alditol acetates (SAWARDEKER et al., 1965) using a Hewlett-Packard 5880 instrument with an Ultra 2 capillary column and a temperature gradient of $150{ }^{\circ} \mathrm{C}(1 \mathrm{~min})$ to $290{ }^{\circ} \mathrm{C}$ at $10{ }^{\circ} \mathrm{C} / \mathrm{min}$. The absolute configurations were determined by GLC of acetylated glycosides with (-)-2-octanol by the method of LEONTEIN et al. (1978) under the same chromatographic conditions as described above.

Methylation analysis: Methylation was carried out with methyl iodide in $\mathrm{Me}_{2} \mathrm{SO}$ in the presence of solid $\mathrm{NaOH}$ (CIUCANU and KEREK, 1984). Hydrolysis was performed as in sugar analysis; partially methylated monosaccharides were reduced with $\mathrm{NaBH}_{4}$, acetylated, and analyzed by GLC-MS on a Hewlett-Packard 5890 chromatograph equipped with a DB-5 
fused-silica capillary column and a Nermag R10-10L mass spectrometer, using a temperature gradient of $160{ }^{\circ} \mathrm{C}(1 \mathrm{~min})$ to $250{ }^{\circ} \mathrm{C}$ at $3{ }^{\circ} \mathrm{C} / \mathrm{min}$. Identification of partially methylated alditol acetates was performed using the data of JANSSON et al. (1976).

NMR spectroscopy: The OPS samples were deuterium-exchanged by freeze-drying three times from $\mathrm{D}_{2} \mathrm{O}$ and then examined in a solution of $99.96 \% \mathrm{D}_{2} \mathrm{O}$. Spectra were recorded using a Bruker DRX-500 spectrometer at $50{ }^{\circ} \mathrm{C}$. Data were acquired and processed using the XWINNMR version 2.1 software. Mixing times of 200 and $100 \mathrm{~ms}$ were used in 2D TOCSY and NOESY experiments, respectively. Chemical shifts are reported with internal acetone $\left(\delta_{\mathrm{H}}\right.$ $\left.2.225, \delta_{\mathrm{c}} 31.45\right)$.

\subsection{Determination of the virulence of two Eca - strains}

\subsubsection{Laboratory experiments}

For the laboratory experiments, the potato cultivar Agria was used. Two different strains of Erwinia carorovora ssp. atroseptica (Eca 2967 and 2962) were compared according to their virulence. The tubers were washed under tap water, dried, immersed in alcohol, flamed and cut with a sterile knife in slices $2.5 \mathrm{~mm}$, thick. The potato slices were placed in Petri dishes.

The two bacterial strains were grown on King's medium B at $27{ }^{\circ} \mathrm{C}$ for $24 \mathrm{~h}$. Each strain was adjusted to an O.D.660 of 0.06 (ca.1.6 X $10^{8} \mathrm{cfu} / \mathrm{ml}$ ) (photometer Spectronic 20, Bausch \& Lomb) and serially diluted. Three different concentrations (5 X 10 4 , $1 \times 10^{4}$ and $\left.5 \times 10^{3}\right)$ from each strain were inoculated $(5 \mu \mathrm{l})$ in the middle of the tuber slices, and $5 \mathrm{ml}$ sterile distilled water were poured into Petri dishes to maintain high humidity. The Petri plates were incubated at $27^{\circ} \mathrm{C}$ and checked daily for 5 days.

\subsubsection{Greenhouse experiments}

Potato tubers of cultivar "Agria" were stored at room temperature in the light for two weeks for sprouting, after which they were planted in pots (ca. $20 \mathrm{~cm}$ diameter) filled with loamy soil mixed with sand containing one tuber in each pot.

The two bacterial strains were cultured and adjusted to a standardized concentration as described above (2.3.1). Four concentrations $\left(10^{8}, 10^{7}, 10^{6}\right.$ and $\left.10^{5} \mathrm{cfu} / \mathrm{ml}\right)$ were used for each strain and three replications for each concentration. The plants were inoculated 21 days after planting with $20 \mu \mathrm{l}$ by a syringe in the stem of the potato plants $2 \mathrm{~cm}$ above soil surface and incubated at $20 / 14^{\circ} \mathrm{C}$ (day /night). The plants were checked daily and the evaluation was 
based on appearance of the blackleg symptom.

In addition, the Eca strains 2962, 2967 and Ecc 436 were tested for pathogenicity on Chinese cabbage and leaves of tomato, potato and tobacco in two experiments. Chinese cabbage, tomato, potato and tobacco plants were planted in in pots (ca. $20 \mathrm{~cm}$ diameter) filled with loamy soil mixed with sand and containing one plant in each pot. The bacterial strains were grown on King's medium B and each strain was adjusted to an O.D ${ }_{660}$ of 0.3 (ca. $10^{9} \mathrm{cfu} / \mathrm{ml}$ ) or 0.06 (ca. $2 \times 10^{8} \mathrm{cfu} / \mathrm{ml}$ ) as described above. The leaves of young plants were injected under a slight pressure with a syringe without needle.

In the first experiment, the bacterial suspensions of ca. $10^{8}, 10^{7}, 10^{6}$ and $10^{5} \mathrm{cfu} / \mathrm{ml}$ of Eca strains 2962, 2967 and Ecc 436 were used to inoculate leaves of tobacco, tomato and Chinese cabbage.

In the second experiment, bacterial suspensions of OD $0.3\left(\mathrm{ca} .10^{9} \mathrm{cfu} / \mathrm{ml}\right)$ and $10^{8} \mathrm{cfu} / \mathrm{ml}$ of the same bacterial strains as listed above were used to inoculate leaves of tomato, potato and Chinese cabbage plants as well as thick leaves of Chinese cabbage heads. Detached thick leaves of Chinese cabbage heads were inoculated with $5 \mu 1$ from the bacterial suspensions, which were injected under slight pressure with a hypodermic needle, and leaves were incubated in plastic boxes (with moist paper at the bottom) at $27^{\circ} \mathrm{C}$ for 5 days to assess symptoms.

\subsection{Determination of pectolytic activity of two Eca - strains}

A) Substrate: solution (1): $0.1 \mathrm{M}$ citric acid (4.2 g in $200 \mathrm{ml} \mathrm{H}_{2} \mathrm{O}$ ); solution (2): $0.1 \mathrm{M} \mathrm{Na}$ citrate (14.7 g in $500 \mathrm{ml} \mathrm{H} \mathrm{H}_{2} \mathrm{O}$ ). Mix $152.5 \mathrm{ml}$ solution (1) and $347.5 \mathrm{ml}$ solution (2), adjust $\mathrm{pH}$ to 5.2 , after warming the buffer to $80{ }^{\circ} \mathrm{C}$, add $5 \mathrm{~g}$ from pectin $\mathrm{N}$ (Roth Co.), mix vigorously with magentic stirrer and adjust $\mathrm{pH}$ to 5.0. This substrate was used within 2 days.

B) At first, the two bacterial Eca strains were precultured on King's medium B (agar) at $27^{\circ} \mathrm{C}$ for $24 \mathrm{~h}$. Then, a loopful of bacteria was inoculated into pectin liquid medium, and incubated in a shaker at $27^{\circ} \mathrm{C}$ for $24 \mathrm{~h}$. From this $2^{\text {nd }}$ preculture bacteria were pelleted by centrifugation, and the pellet was suspended in $0.01 \mathrm{M} \mathrm{MgSO}_{4}$ and adjusted to an $\mathrm{OD}_{660}$ of 0.06 . From these bacterial suspensions $0.1 \mathrm{ml}$ were inoculated into Erlenmyer flasks with pectin liquid medium and incubated in the shaker at $110 \mathrm{rpm}$ (Clim-O-shake, Firma Kühner, Basel, Switzerland) for $48 \mathrm{~h}$. At different time intervals $(0 \mathrm{~h}, 6 \mathrm{~h}, 12 \mathrm{~h}, 18 \mathrm{~h}, 24 \mathrm{~h}, 30 \mathrm{~h}, 42 \mathrm{~h}$ and $48 \mathrm{~h})$ samples of 10 $\mathrm{ml}$ were taken, centrifuged at $6000 \mathrm{~g} / 10 \mathrm{~min}$, and the supernatant stored at $4{ }^{\circ} \mathrm{C}$ until use. When the last sample had been taken, each supernatant was mixed with $30 \mathrm{ml}$ substrate 
solution (A) supplemented by $5 \mathrm{ml} 0.01 \mathrm{M} \mathrm{CaCl}_{2}$ and $0.02 \%$ sodium azide. The reaction mixtures were incubated for $24 \mathrm{~h}$ at $37{ }^{\circ} \mathrm{C}$, after which the viscosity was measured by a viscosimeter.

At the same time intervals $(0-48 \mathrm{~h})$ bacterial growth was determined. $0.5 \mathrm{ml}$ from the bacterial culture were serially diluted, and $0.1 \mathrm{ml}$ were plated onto King's medium B and incubated at $27^{\circ} \mathrm{C}$ for $24 \mathrm{~h}$.

Viscosity of the reaction mixtures was determined with a falling ball viscosimeter (Haake, Germany). For this purpose, $20 \mathrm{ml}$ culture fluid were used. The time in seconds required for the ball to pass through a definite distance in a tilted (100) cylindrical tube was recorded. The readings obtained were converted into the units of $\mathrm{mPa} X$ sec by the formular equation (ELSHOUNY, 1993), where $1 \mathrm{mPa} X \mathrm{sec}=1 \mathrm{cP}$ (Centipoise):

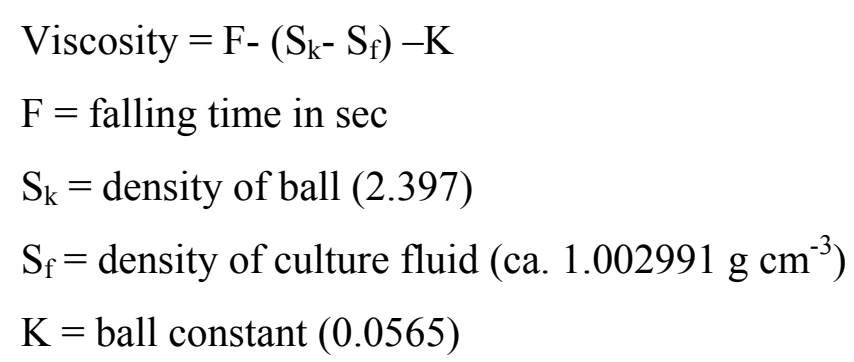

All viscosity measurements were taken at room temperature. The sample was allowed to equilibrate for $2 \mathrm{~min}$ in the viscosimeter before the viscosity was determined.

\subsubsection{Multiplication of two Eca - strains in pectin and King's B liquid medium}

The bacterial strains (GSPB Eca 2967 and GSPB Eca 2962) were grown as described in 2.4. The next step was a preculture in King's B or pectin liquid medium, in a shaker at $27^{\circ} \mathrm{C}$ at 110 rpm (Clim-O-shake, Firma Kühner, Basel, Switzerland) for 24 h. Bacterial cells (10 ml) were centrifuged at $6000 \mathrm{~g} / 10 \mathrm{~min}$, and the pellet was dissolved in $10 \mathrm{ml} 0.01 \mathrm{M} \mathrm{MgSO}_{4}$ to adjust the bacterial concentration to an O.D.660 of 0.06 , corresponding to ca.3.3 X10 ${ }^{7} \mathrm{cfu} \mathrm{ml}$ (Eca 2962) and $3.5 \times 10^{7} \mathrm{cfu} / \mathrm{ml}$ (Eca 2967). The suspensions were serially diluted and $0.1 \mathrm{ml}$ from the dilution $1: 10^{5}$ were plated onto King's medium B and incubated at $27{ }^{\circ} \mathrm{C}$ for 24 h. $0.1 \mathrm{ml}$ from the same concentration were inoculated into an Erlenmeyer flask and shaken (110 rpm) at $27^{\circ} \mathrm{C}$ for different times $(0 \mathrm{~h}, 6 \mathrm{~h}, 12 \mathrm{~h}, 24 \mathrm{~h}, 30 \mathrm{~h}, 42 \mathrm{~h}$ and $48 \mathrm{~h})$. The bacterial growth was determined by dilution plating.

\subsubsection{Determination of bacterial growth of two Eca strains using the semi- selective medium (CVPM)}

The two bacterial strains (2967 and 2962) were grown on King's medium B and incubated at 
$27{ }^{\circ} \mathrm{C}$ for $24 \mathrm{~h}$. The bacterial growth was scraped off in $0.01 \mathrm{M} \mathrm{MgSO}_{4}$, and adjusted to an O.D.660 of 0.06 (ca. $10^{8} \mathrm{cfu} / \mathrm{ml}$ ). The bacterial suspensions were spot-inoculated onto the semiselective medium and incubated at $27^{\circ} \mathrm{C}$ for $24 \mathrm{~h}$. 
3

\section{RESULTS}

3.1

Analysis of lipopolysaccarides (LPS)

\subsubsection{Characterization by SDS-PAGE}

The results of silver staining after SDS-PAGE are shown in Fig. 1. Both preparations from Eca 2967 and Ecc 436 (lane nos. 4, 5 and 6) were found to give typical lipopolysaccharide electrophoretic patterns with the ladder-like appearance due to the LPS-O-chain. In contrast, the LPS extracted from the Eca strain 2962 (lane nos. 2 and 3) did not show the typical ladder-patterns, indicating a "rough" LPS.

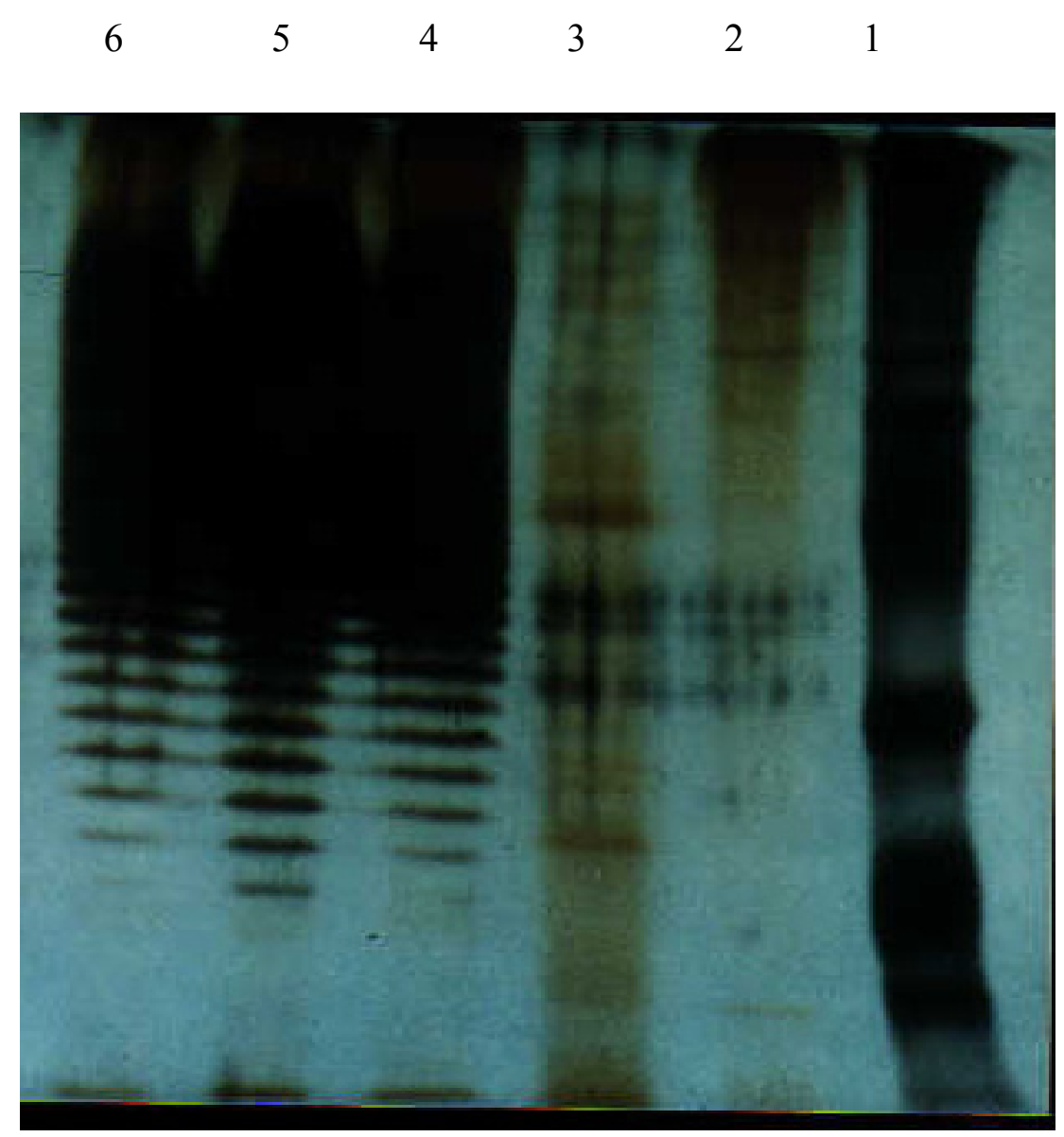

Fig. 1: SDS-PAGE of lipopolysaccharides from Erwinia spp.. The samples were run on a 10\% acrylamide gel and stained as described in Chapter II, 2.5.3. Lanes: (1) Marker VIII, (2 and 3) Eca 2962, (4) Ecc 436, (5) Eca 2967, (6) Ecc 436. Into each slot $10 \mu$ LPS-solution (containing 0.2-0.5 mg/ml water) were added. Only no. 2 contained $5 \mu 1$. 


\subsubsection{Structure of the LPS-O-chain of Ecc (GSPB 436)}

The O-polysaccharide of Erwinia carotovora ssp. carotovora GSPB 436 was studied by sugar and methylation analysis and ${ }^{1} \mathrm{H}$ and ${ }^{13} \mathrm{C}$ NMR spectroscopy, including 2D COSY, TOCSY, NOESY and $\mathrm{H}$-detected ${ }^{1} \mathrm{H},{ }^{13} \mathrm{C}$ heteronuclear multiple-quantum coherence (HMQC) experiments in the laboratory of Dr. Knirel (see subchapter 2.2). The polysaccharide was found to contain L-rhamnose, D-glucose and D-fucose in the ratio 2:1:1 (Fig. 2), and the following structure of the tetrasaccharide repeating unit was established:

$\rightarrow 3)-\beta-L-R h a p-(1 \rightarrow 4)-\alpha-L-R h a p-(1 \rightarrow 3)-\alpha-D-F u c p-(1 \rightarrow$

3
$\uparrow$
1
$\alpha-D-G l c p$

Fig. 2: Structure of Ecc GSPB 436 O-side-chain (after Knirel, with ${ }^{13} \mathrm{C}$ NMR- spectroscopy analysis)

\subsubsection{Structure of the LPS-O-chain of Eca (GSPB 2967)}

The structure of the LPS-O-chain of E. carotovora ssp. atroseptica (strain GSPB 2967) was only preliminary determined by Dr. Knirel and coworkers. Accordingly, the O-chain has a pentasaccharide repeating unit containing three galactose residues, one rhamnose and a new higher branched sugar (similar to caryophyllose). The higher branched sugar showed some similarity to caryophyllose from Pseudomonas caryophylli (ADINOLFI et al., 1995 a and 1995 b), but has less carbons in the side chain.

\subsection{Virulence test of two Erwinia carotovora spp. atroseptica strains on potato tuber slices and Chinese cabbage}

\subsubsection{Tests on potato slices in Petri dishes}

Results in Table 1 show that the strain Eca 2967 was highly virulent on potato slices, because 3 days after inoculation with $5 \times 10^{4} \mathrm{cfu} / \mathrm{ml}$ and 5 days after inoculation with $1 \times 10^{4} \mathrm{cfu} / \mathrm{ml}$ nearly the whole potato tissue was macerated. On the other hand, strain Eca 2962 was very weakly virulent since only the highest concentration of $5 \times 10^{4} \mathrm{cfu} / \mathrm{ml}$ caused medium rotting of potato slices after 4-5 days. 
Table 1: Virulence of two Eca strains on potato tuber slices

\begin{tabular}{|c|c|c|c|c|c|c|}
\hline \multirow[t]{2}{*}{ Day } & \multicolumn{3}{|c|}{$\begin{array}{c}\text { Bacterial concentration } \\
\text { Eca } 2967\end{array}$} & \multicolumn{3}{|c|}{$\begin{array}{c}\text { Bacterial concentration } \\
\text { Eca } 2962\end{array}$} \\
\hline & $5 \times 10^{4}$ & $1.0 \times 10^{4}$ & $5.0 \times 10^{3}$ & $5 \times 10^{4}$ & $1.0 \times 10^{4}$ & $5.0 \times 10^{3}$ \\
\hline 1 & $1.3 *$ & 0.5 & 0 & 0.2 & 0 & 0 \\
\hline 2 & 2.0 & 0.7 & 0 & 0.3 & 0 & 0 \\
\hline 3 & Whole & 1.2 & 0 & 0.7 & 0 & 0 \\
\hline 4 & Whole & 2.0 & 0 & 1.0 & 0 & 0 \\
\hline 5 & Whole & 2.5 & 0 & 1.2 & 0 & 0 \\
\hline
\end{tabular}

* = Diameter of rotted tissue in $\mathrm{cm}$

\subsubsection{Tests on potato plants in pots in the greenhouse}

Fig. 3 shows a strong difference in symptoms caused by the two strains. Typical blackleg symptoms appeared on potato cultivar Agria one week after inoculation with strain Eca 2967 at bacterial concentrations of $10^{8}, 10^{7}$ and $10^{6} \mathrm{cfu} / \mathrm{ml}$. In contrast, none of the four bacterial concentrations tested of Eca 2962 had caused disease symptoms within one month after inoculation. It was concluded from this experiment that the Japanese strain Eca 2962 was nearly avirulent as incitant of potato blackleg.

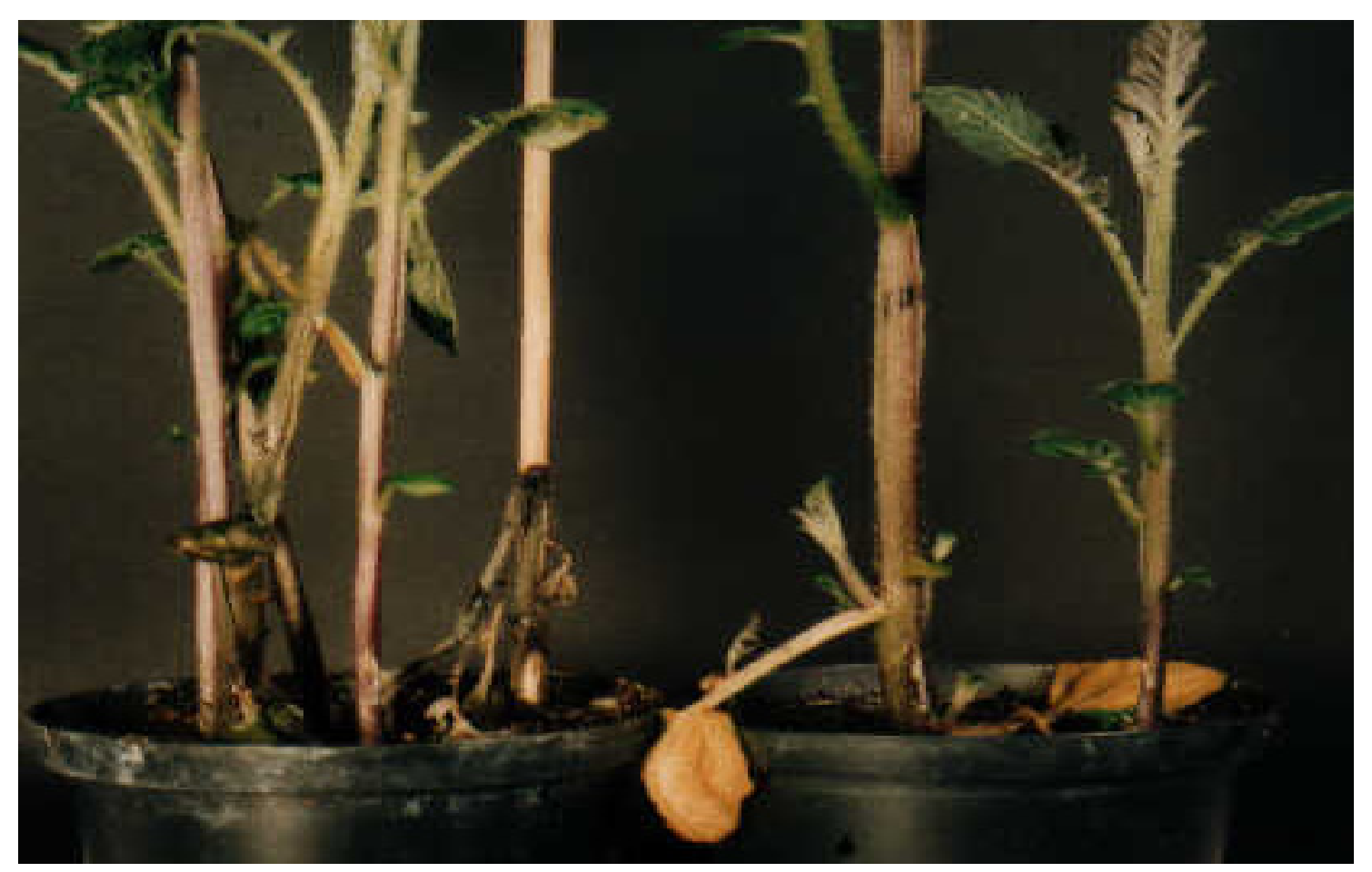

Fig. 3: Blackleg symptoms on potato plants (cultivar Agria) after inoculation with $10^{8} \mathrm{cfu} / \mathrm{ml}$. Only strain Eca 2967 (left) caused typical blackleg symptoms while inoculation with strain Eca 2962 (right) showed only small necrotic spots due to the wounding by the inoculation precedure. 


\subsubsection{Tests on leaves of Chinese cabbage, tomato, potato and tobacco and on thick leaves of Chinese cabbage heads}

Results (Fig. 4) show that in the first experiment, the Japanese strain Eca 2962 caused slight necrotic symptoms on leaves of tomato plants only, with a high bacterial concentration (ca. $10^{8}$ cfu/ml), whereas the other bacterial concentrations of Eca 2967 and Ecc 436 did not cause any symptoms on all the plant leaves tested (tomato, potato, tobacco). The results of the second experiment, (Figs. 5, 6 and 7) show that the higher concentrations $\left(10^{9} \mathrm{cfu} / \mathrm{ml}\right)$ of Eca 2962 and Eca 2967 caused unspecific necrotic spots on leaves of tomato (Fig. 5) and potato (Fig. 6) but no symptoms on young leaves of Chinese cabbage (Fig. 7) and tobacco (not shown). On tomato leaves, the symptoms caused by the Japanese strain Eca 2962 appeared to be a little bit stronger than those caused by Eca 2967 (Fig. 5). On thick leaves of Chinese cabbage extended soft rotting was caused by $10^{9} \mathrm{cfu} / \mathrm{ml}$ of Eca 2967, but only limited soft rot spots by Eca 2962 (Fig. 8). With the lower concentration of $10^{8} \mathrm{cfu} / \mathrm{ml}$, the Japanese strain 2962 caused mostly necrotic spots with a small soft rotting border, while Eca 2967 caused large confluent soft rotted areas (Fig. 9). It was concluded from these tests that the Japanese strain was a very weak soft rot pathogen.

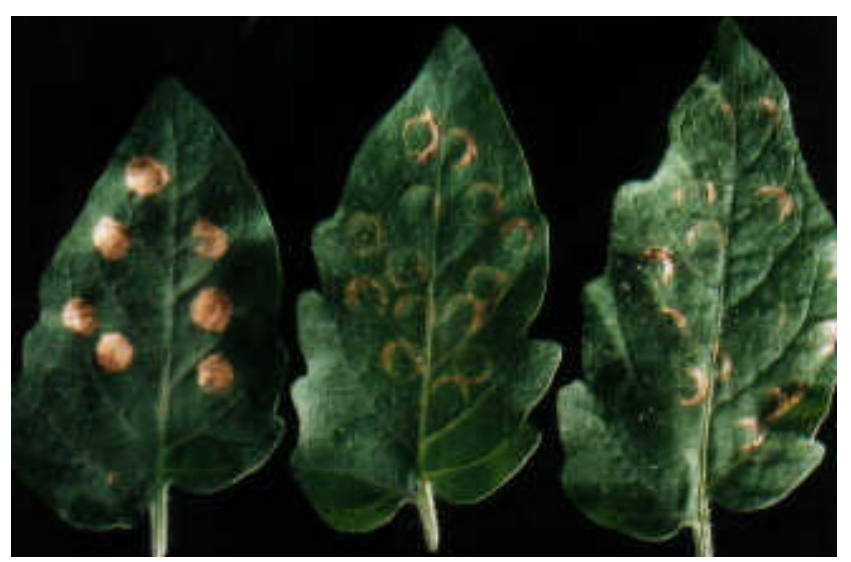

Fig. 4: Spot symptoms on tomato leaves after inoculation with $10^{8} \mathrm{cfu} / \mathrm{ml}$. Only strain Eca 2962 (left) caused necrotic symptoms while no symptoms were observed with Eca 2967 and Ecc 436, respectively, 5 days after inoculation. 


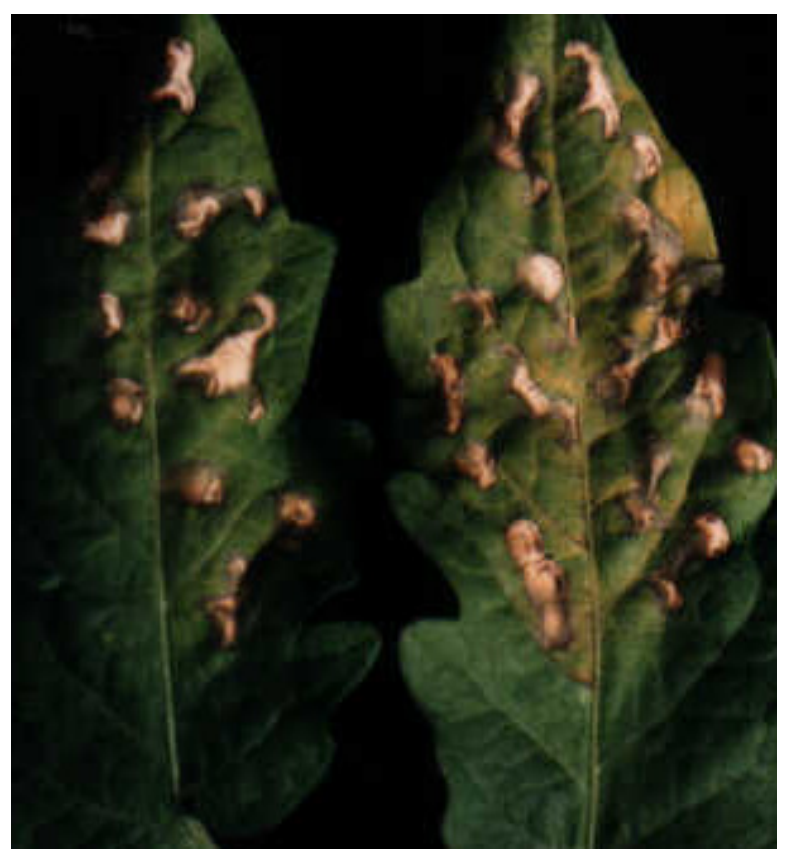

Fig. 5: Necrotic spots on tomato leaves after inoculation with $10^{9} \mathrm{cfu} / \mathrm{ml}$. Strain Eca 2962 (right) caused a little bit stronger necrotic spots with more yellowing than that caused by Eca strain 2967 (left).

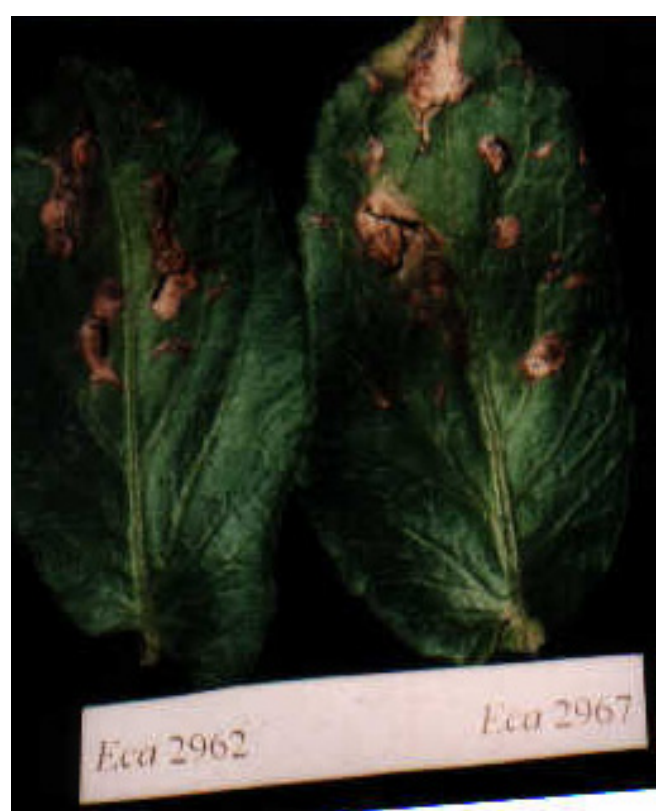

Fig. 6: Necrotic spots on potato leaves after inoculation with $10^{9} \mathrm{cfu} / \mathrm{ml}$. Both Eca strains (2962 and 2967) showed similar necrotic symptoms 3 days after inoculation. 


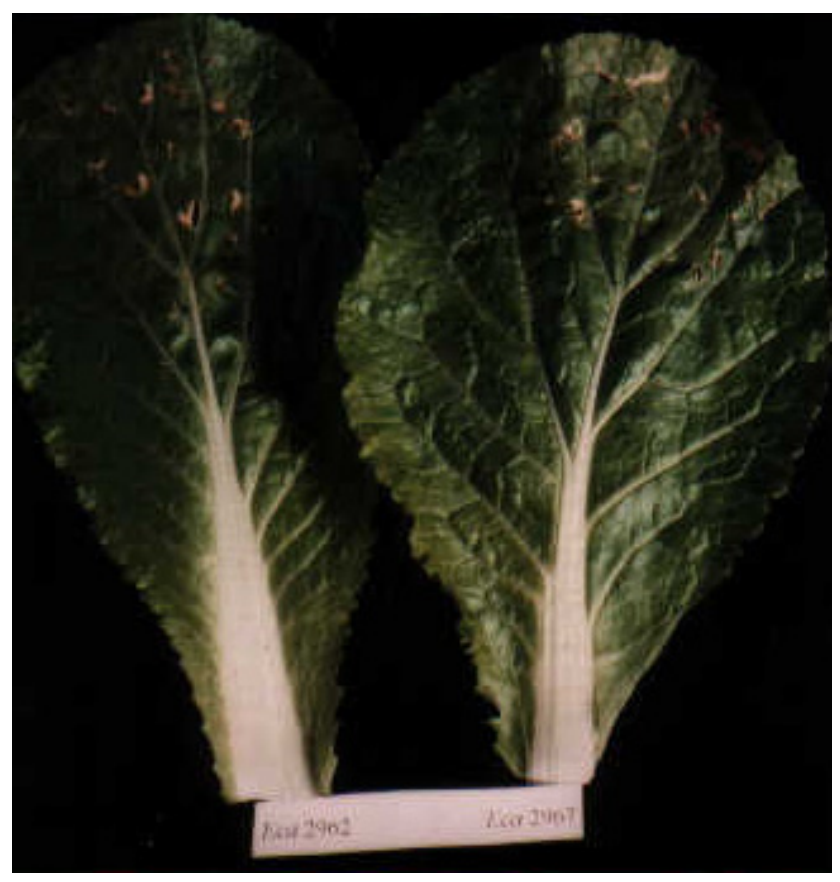

Fig. 7: Leaves of Chinese cabbage inoculated with $10^{9} \mathrm{cfu} / \mathrm{ml}$. Both Eca strains (2962 and 2967) had caused no symptoms 3 days after inoculation.

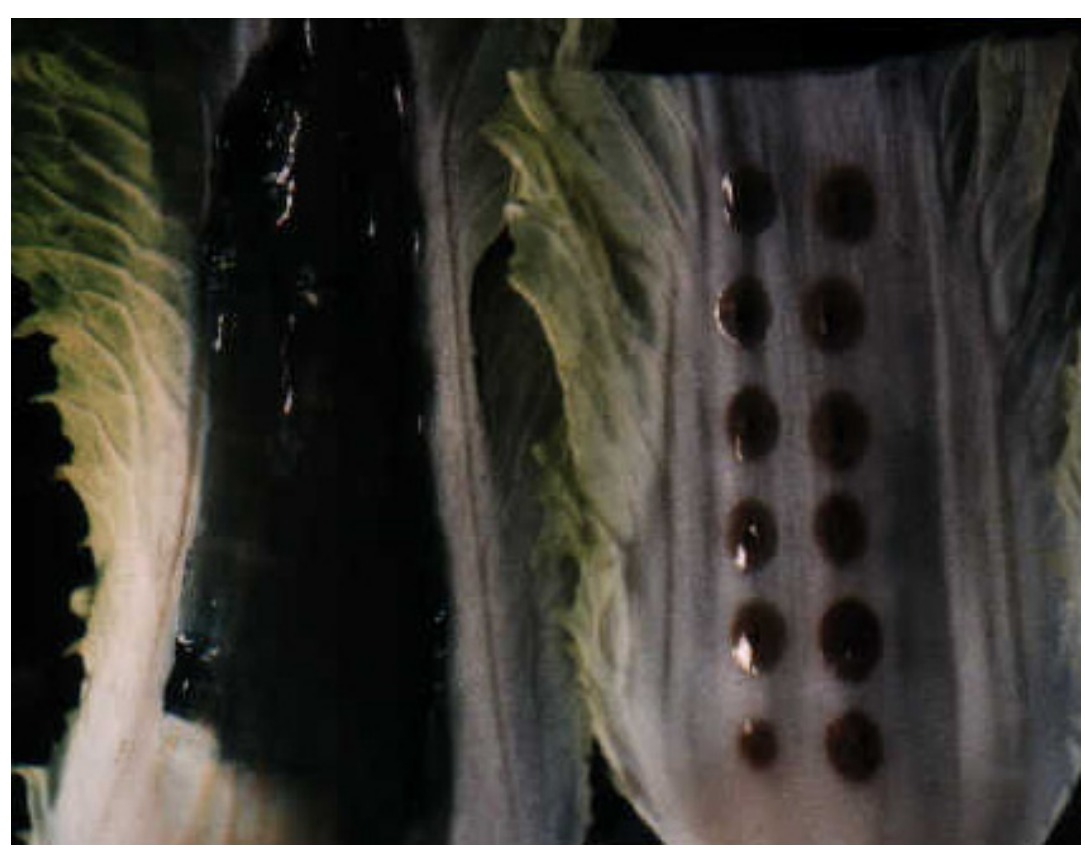

Fig. 8: Soft rot symptoms on Chinese cabbage after inoculation with $10^{9} \mathrm{cfu} / \mathrm{ml}$. Strain Eca 2967 (left) caused extended soft rotting while strain Eca 2962 (right) provoked limited soft rot spots, 2 days after inoculation. 


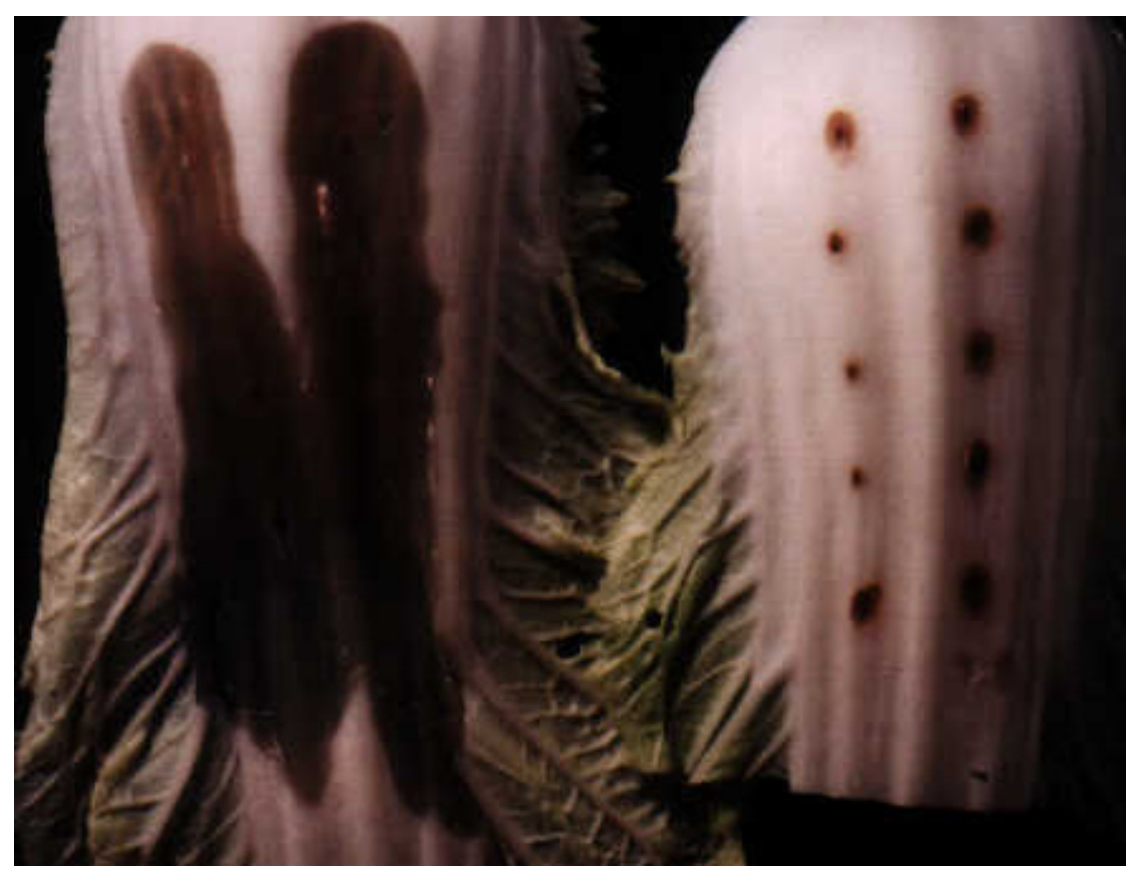

Fig. 9: Soft rot symptoms on Chinese cabbage after inoculation with $10^{8} \mathrm{cfu} / \mathrm{ml}$. Strain Eca 2967 (left) caused large confluent soft rotted areas while strain Eca 2962 (right) caused mostly necrotic spots with a small soft rotting border, 2 days after inoculation.

\subsection{Pectolytic activity}

As shown in Fig. 10 and listed in Tables 2 and $\mathbf{3}$ the supernatant of the German strain (GSPB 2967) possessed a much higher pectinase activity than that of the Japanese strain (GSPB 2962) from 6 to $30 \mathrm{~h}$ after inoculation of the pectin medium. However, when the pectinase activity in the supernatant was calculated per bacterial cell it turned out that the pectolytic activity of the Japanese strain was similar to that of the German strain, the difference between the two strains not being significant. On the other hand, the German strain showed maximum detectable pectinase activity clearly after 18, the Japanese strain only after $42 \mathrm{~h}$. Therefore, in further experiments the growth of the two strains was compared in and on different media (chapter $3.4)$. 


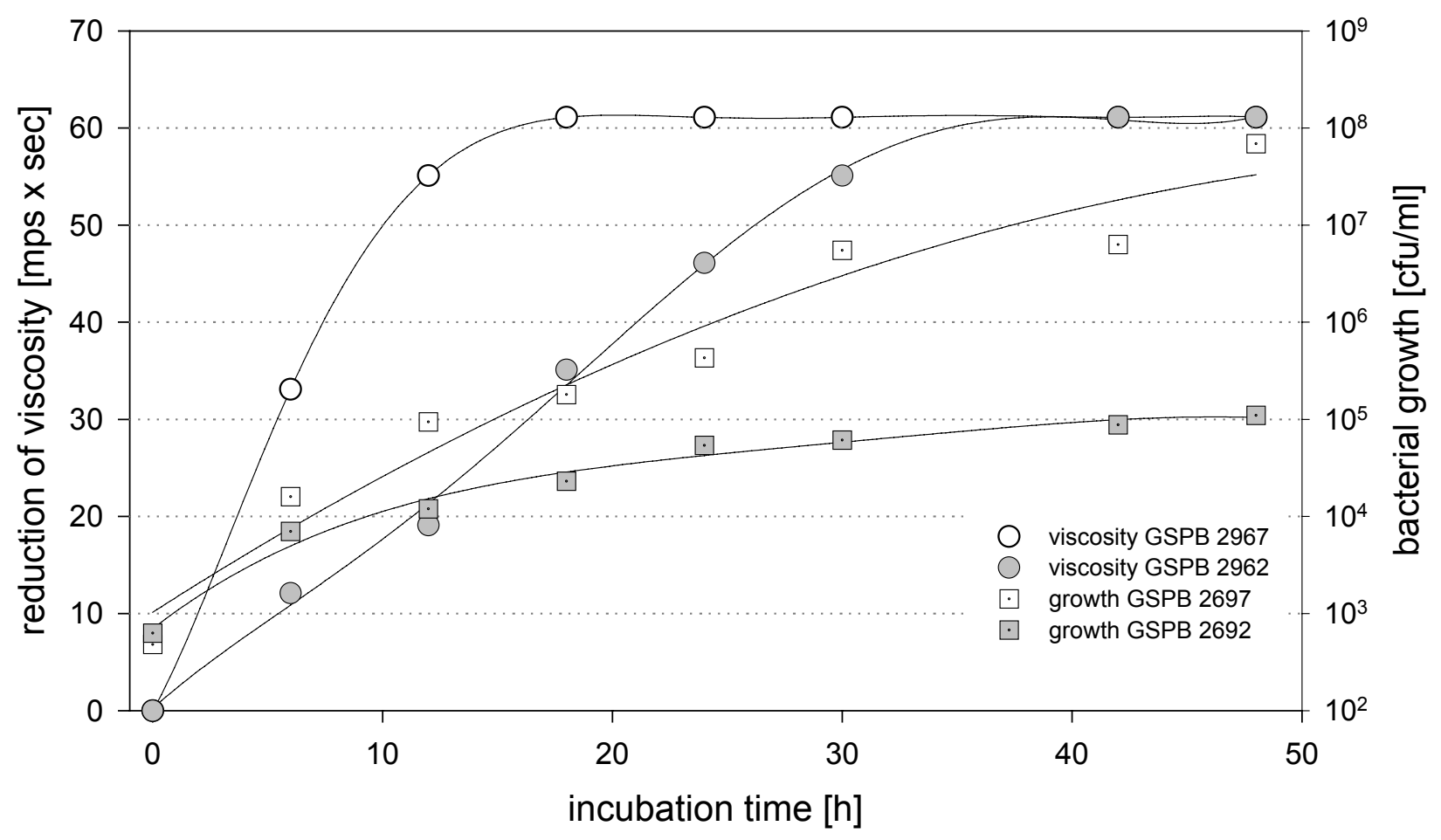

Fig. 10: Pectinase activity and bacterial growth from $0-48 \mathrm{~h}$ in a pectin liquid medium

Table 2: Pectolytic activity of Erwinia carotovora ssp. atroseptica, German strain (GSPB 2967)

\begin{tabular}{|l|l|c|c|c|c|}
\hline Incubation time & $\begin{array}{l}\text { Bacterial } \\
\text { concentration in the } \\
\text { pectin liquid medium } \\
\text { (cfu/ml) }\end{array}$ & $\begin{array}{c}\text { Speed of fall in } \\
\text { seconds/5 cm }\end{array}$ & $\begin{array}{c}\text { Viscosity } \\
\text { (mpa X sec) }\end{array}$ & $\begin{array}{c}\text { Reduction } \\
\text { of viscosity in } \\
\text { (mpa X sec) }\end{array}$ & $\begin{array}{c}\text { Pectinase activity } \\
\text { per bacterial cell } \\
\text { (mpa X sec) }\end{array}$ \\
\hline $0 \mathrm{~h}$ & $4.8 \times 10^{2}$ & 65 & 61.6 & 0 & 0.0 \\
\hline $6 \mathrm{~h}$ & $1.6 \times 10^{4}$ & 32 & 28.5 & 33.1 & $2.1 \times 10^{-3}$ \\
\hline $12 \mathrm{~h}$ & $9.4 \times 10^{4}$ & 10 & 6.5 & 55.1 & $0.59 \times 10^{-3}$ \\
\hline $18 \mathrm{~h}$ & $1.8 \times 10^{5}$ & 3.0 & 0.46 & 61.1 & $0.34 \times 10^{-3}$ \\
\hline $24 \mathrm{~h}$ & $4.3 \times 10^{5}$ & 3.0 & 0.46 & 61.1 & $0.14 \times 10^{-3}$ \\
\hline $30 \mathrm{~h}$ & $5.5 \times 10^{6}$ & 3.0 & 0.46 & 61.1 & $0.01 \times 10^{-3}$ \\
\hline $42 \mathrm{~h}$ & $6.3 \times 10^{6}$ & 3.0 & 0.46 & 61.1 & $0.01 \times 10^{-3}$ \\
\hline $48 \mathrm{~h}$ & $6.9 \times 10^{7}$ & 3.0 & 0.46 & 61.1 & $0.001 \times 10^{-3}$ \\
\hline
\end{tabular}


Table 3: Pectolytic activity of Erwinia carotovora ssp. atroseptica, Japanese strain (GSPB2962)

\begin{tabular}{|l|c|c|c|c|c|}
\hline Incubation time & $\begin{array}{c}\text { Bacterial } \\
\text { concentration in the } \\
\text { pectin liquid medium } \\
\text { (cfu/ml) }\end{array}$ & $\begin{array}{c}\text { Speed of fall in } \\
\text { seconds/5 cm }\end{array}$ & $\begin{array}{c}\text { Viscosity } \\
\text { (mpa X sec) }\end{array}$ & $\begin{array}{c}\text { Reduction of } \\
\text { viscosity in } \\
\text { (mpa X sec) }\end{array}$ & $\begin{array}{c}\text { Pectinase activity per } \\
\text { bacterial cell } \\
\text { (mpa X sec) }\end{array}$ \\
\hline $0 \mathrm{~h}$ & $6.3 \times 10^{2}$ & 65 & 61.6 & 0 & 0.0 \\
\hline $6 \mathrm{~h}$ & $7.0 \times 10^{3}$ & 53 & 49.5 & 12.1 & $1.73 \times 10^{-3}$ \\
\hline $12 \mathrm{~h}$ & $1.2 \times 10^{4}$ & 46 & 42.5 & 19.1 & $1.59 \times 10^{-3}$ \\
\hline $18 \mathrm{~h}$ & $2.3 \times 10^{4}$ & 30 & 26.5 & 35.1 & $1.53 \times 10^{-3}$ \\
\hline $24 \mathrm{~h}$ & $5.4 \times 10^{4}$ & 19 & 15.5 & 46.1 & $0.85 \times 10^{-3}$ \\
\hline $30 \mathrm{~h}$ & $6.1 \times 10^{4}$ & 10 & 6.5 & 55.1 & $0.9 \times 10^{-3}$ \\
\hline $42 \mathrm{~h}$ & $8.8 \times 10^{4}$ & 3.0 & 0.46 & 61.1 & $0.69 \times 10^{-3}$ \\
\hline $48 \mathrm{~h}$ & $1.1 \times 10^{5}$ & 3.0 & 0.46 & 61.1 & $0.56 \times 10^{-3}$ \\
\hline
\end{tabular}

\subsection{Mutiplication of $E c a$-strains in/on different media}

\subsubsection{In pectin medium}

As shown in Fig. 11 the German strain Eca 2967 multiplied faster in liquid pectin medium than the Japanese strain Eca 2962. Thus, GSPB 2967 reached $10^{7} \mathrm{cfu} / \mathrm{ml}$ after 48 h, whereas GSPB 2962 reached only $10^{5} \mathrm{cfu} / \mathrm{ml}$ after $48 \mathrm{~h}$. It might be concluded from these results that the growth of the German strain was much faster than that of the Japanese strain, because the German strain had a higher pectinase activity. In order to proof this hypothesis, bacterial growth on King's medium B (with glycerol as carbon source) was also determined, see subchapter 3.4.2. 


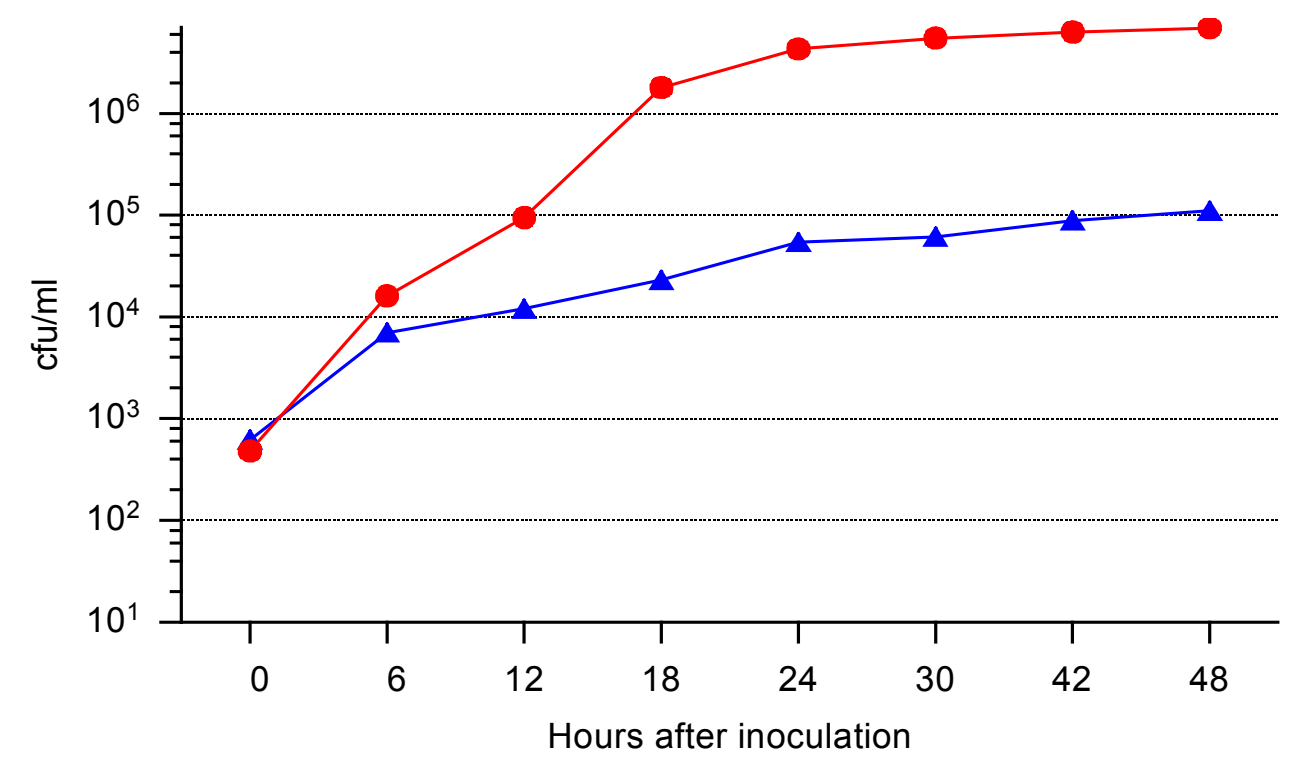

Fig. 11: Growth of the two Eca strains 2967 and 2962 in liquid pectin medium.

\subsubsection{In KB medium}

Unexpectedly, an even higher difference in the multiplication rate of strains 2967 and 2962 was observed on King's medium B than in pectin medium (see Fig. 12). For instance, more than $10^{9} \mathrm{cfu} / \mathrm{ml}$ were determined for the strain $296748 \mathrm{~h}$ after inoculation, but only $10^{6} \mathrm{cfu} / \mathrm{ml}$ for strain 2962. It was concluded from these results that the slower growth of the „rough“ Eca strain 2962 in King's medium B compared to the pectin medium was not due to a low pectinase activity of strain 2967. Obviously, strain 2962 was characterized by a slower growth vigour than strain 2967, and the lower pectinase activity may be a consequence of this partial deficiency. The question whether the complete loss of the LPS-O-chain is in some way connected with the lower growth vigour remains unanswered. 


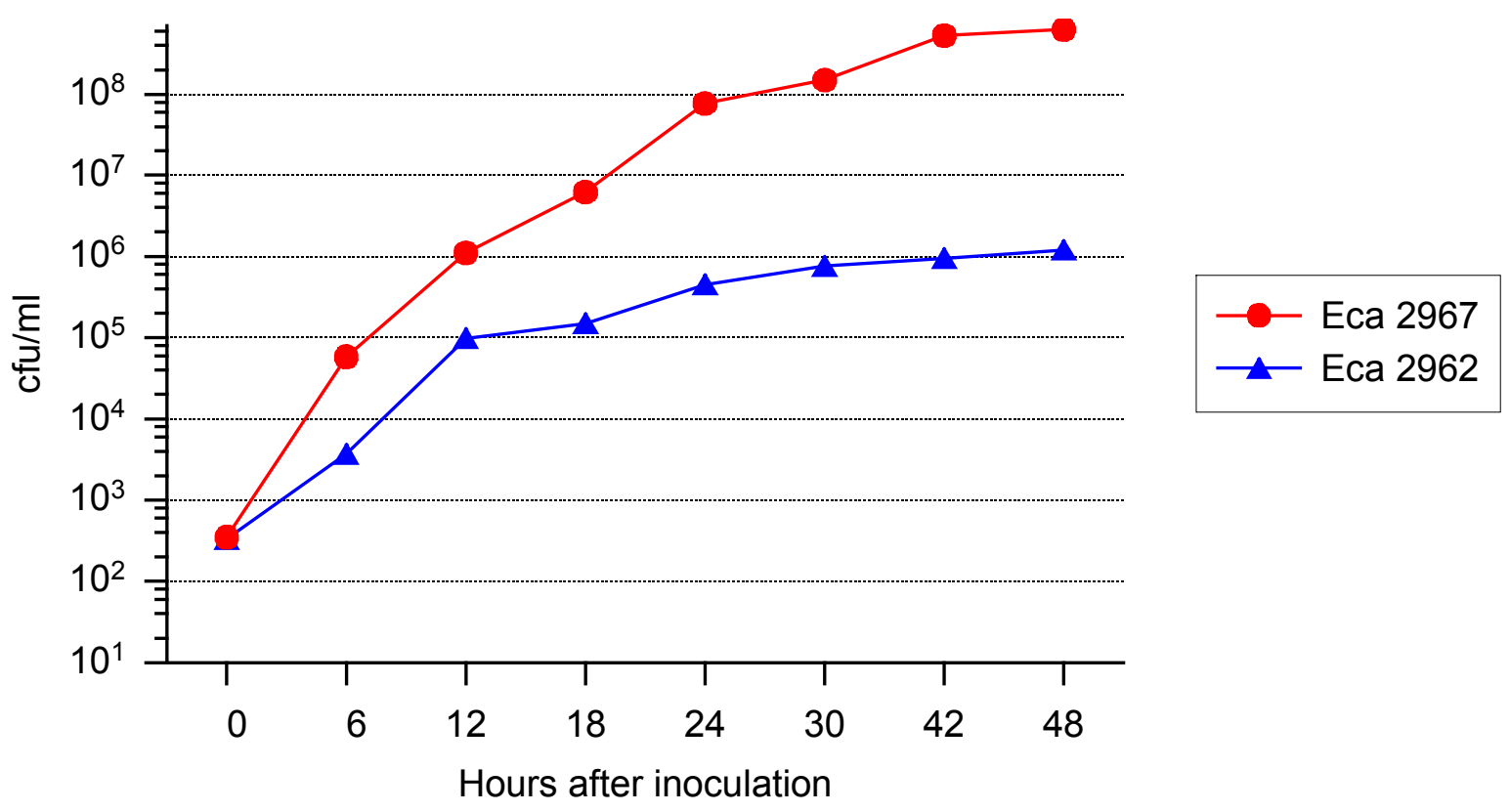

Fig. 12: Growth of the two Eca strains 2967 and 2962 in liquid KB medium

\subsubsection{Comparison of the growth of two Eca strains on the CVPM medium}

As shown in Fig. 13 the cavities formed on CVPM medium by the German strain Eca 2967 were broader and deeper compared to those formed by the Japanese strain Eca 2962, the cavities of which were shallow and smaller. Also, these results indicated that the German strain multiplied faster on the semi-selective medium than the Japanese strain. Therefore, the cavities were bigger and broader.

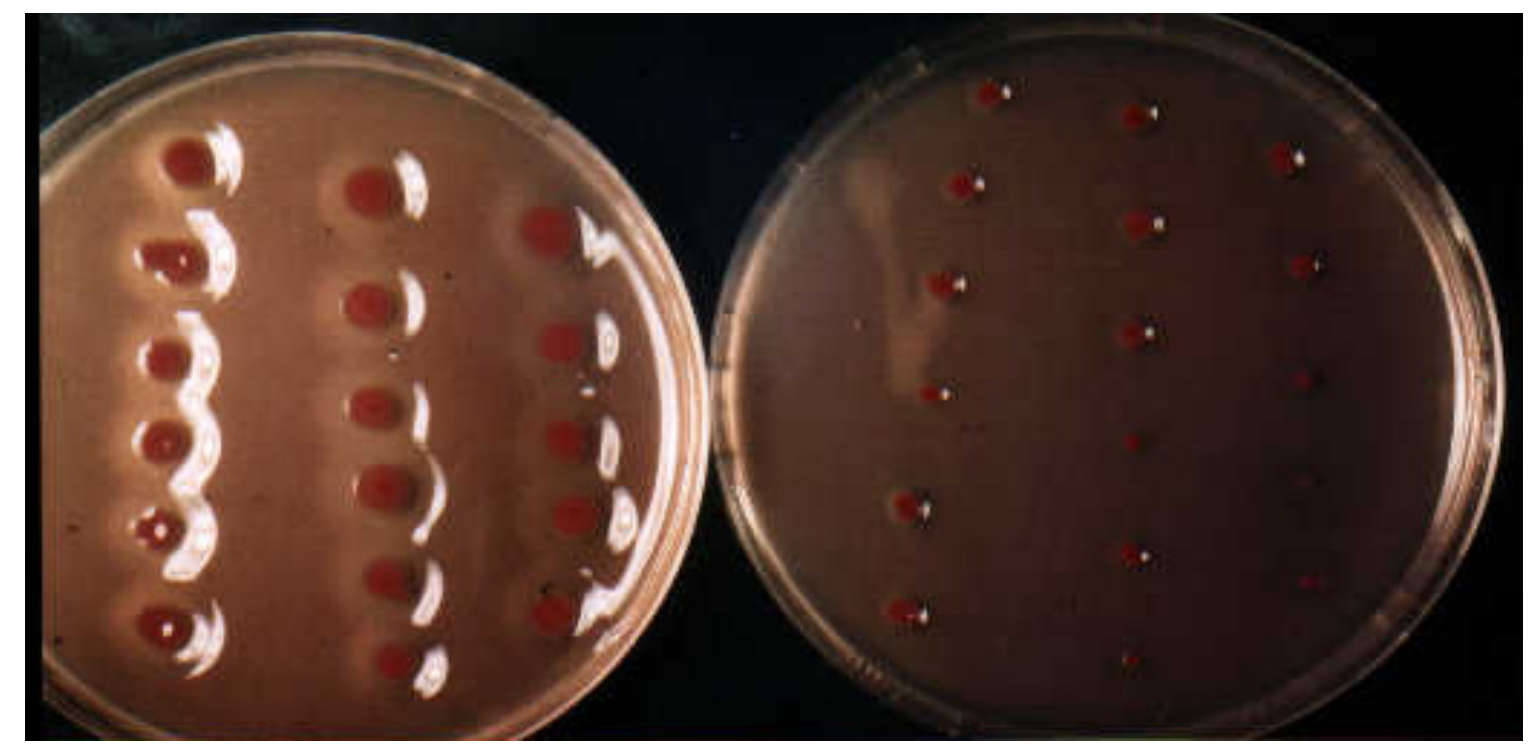

Fig. 13: Cavity formation by the two Eca strains on CVPM medium. Strain Eca 2967 formed deep and broad cavities (left) while strain Eca 2962 showed shallow and small cavities (right). 


\section{DISCUSSION}

It was suggested that bacterial phytopathogenicity is affected by the cell wall lipopolysaccharides (LPS) (CHATTERJEE and STARR, 1980). LPS of Gram-negative bacteria are composed of three genetically and structurally distinct regions; lipid A, core region and O-specific chain. The preparation of largely intact, i.e. biologically active lipid A from enterobacteriaceae was possible because of the acid-labile ketosidic linkage between the core oligosaccharide and lipid A (ZÄHRINGER et al., 1998). The lipid A extracted from Erwinia carotovora strain GSPB 2962 (=Ferm P-7576) consists of a B-1',6-linked glucosamine disaccharide substituted with phosphate at position 4 , and carries ester- and amide-bound fatty acids similar to the lipid A from other Gram-negative bacteria (FUKUOKA et al., 1992).

It has been assumed that parts of the core oligosaccharide may contribute to endotoxic activities and in fact it is now generally accepted that the core oligosaccharide may modulate the biological activity of lipid A (RIETSCHEL et al., 1993). DE BOER et al. (1985) found that all R-type core LPS structures from 16 Erwinia carotovora strains contain the same components as several enterobacteriaceae such as KDO, glucosamine, heptose, glucose and usually galactose. Thus, it appears that Erwinia carotovora has an LPS core structure very similar to that of animal and human pathogens classified in genera such as Salmonella, Shigella and Escherichia. FUKUOKA et al. (1997) studied the complete structure of R-type lipopolysaccharide of Erwinia carotovora strain Ferm P- 7576 and found the structure to differ from that reported for Erwinia carotovora strain B374 (SANDULACHE and PREHM, 1985).

The O-antigenic polysaccharides (O-specific chain) are present in S-strains of Gramnegative bacteria, which form "smooth" colonies. Therefore, the fine structure of the O-chain is the principal antigenic determinent of the enterobacterial LPS. Also, for the heterogeneous group of Erwinia carotovora subspecies and strains it was proposed that serogroup specificity is a function of the O-chain structure (DE BOER \& Mc NAUGHTON, 1987), because strains with the same LPS composition differed in serogroup affinity. Thus, the strains in serogroups IX and XVIII contain rhamnose but these strains and their purified LPS do not cross-react serologically (DE BOER et al., 1985). These results indicate that Erwinia carotovora serogroups probably are based on the LPS-O-chains. It is clear that the Erwinia spp. LPS will electrophorese in the same characteristic fashion as LPS from other bacterial species (SIDBERRY et al., 1985). Thus, the fastest migrating bands on the silver-stained acrylamide gel in the here reported studies (Fig. 1) indicated a clear difference between the two strains (S- 
type), which have an O-specific chain in comparison with the other strain (R-type), which does not have an O-specific chain.

Notwithstanding extensive studies on different serogroups of Erwinia carotovora which were attributed to different LPS-structures, the chemical structure of the O-chains of Erwinia carotovora -LPS has never been elucidated.

The aim of this study was to elucidate and investigate the chemical structure of LPS-O-chain from Erwinia strains, and to evaluate its possible role in virulence of Eca strains.

\subsection{Structure of the LPS-O-chain of Erwinia carotovora ssp. carotovora}

The chemical composition of the LPS-O-chain of Gram-negative bacteria has been assumed to be highly diverse and to determine the immunospecificity of bacterial species, subspecies and serotypes (KNIREL and KOCHETKOV, 1994). Each serologically distinguishable S-strain of bacteria produces an $\mathrm{O}$-specific polysaccharide with its own unique structure and intraspecies serological classification. The resulting typing schemes of S-strains of bacteria which are important for epidemology are based on the specificity of the O-chains (KNIREL and KOCHETKOV, 1994; KNIREL and ZDOROVENKO, 1997). For instance, lipopolysaccharides of Salmonella T I (a transient variant from rough to smooth form) are characterized by the presence of polysaccharide chains of two types, which are the homopolymers D-ribofuranose and D-galactofuranose (KNIREL and KOCHETKOV, 1994). The O-chains appear to be specific for certain pathovars of Pseudomonas syringae or Xanthomonas campestris (KAMIUNTEN and FUJTA, 1990; ZDOROVENKO et al., 1992; YAKOVLEVA et al., 1994; OVOD et al., 1996, 1997) and may play a role in host specificity (RUDOLPH, 1999).

Our studies revealed for the first time the chemical structure for the LPS-O-chain from Erwinia carotovora ssp. carotovora (SENCHENKOVA et al., 2001). Earlier studies elucidated only the chemical structure of the core region and lipid A of Erwinia carotovora (FUKUOKA et al., 1992, 1997). These authors analysed the LPS of strain Eca 2962 (Ferm P- 7576), which is an R-type and does not contain the O-chain. Our studies confirmed that the LPS of Eca 2962 strain did not show the typical ladder-like pattern of the LPS-O-chain, and this was similar to those obtained by FUKUOKA et al. (1997). Therefore, we selected the highly virulent strains Eca 2967 and Ecc 436 possessing smooth LPS to elucidate the structures of their LPS-Ochains. The results obtained by SENCHENKOVA et al. (2001) indicated that the chemical structure of the LPS-O-chain of Erwinia carotovora ssp. carotovora strain GSPB 436 was 
similar to that of Pseudomonas fluorescens biovar A (strain IMV 472) (KNIREL et al., 1994) as follows:

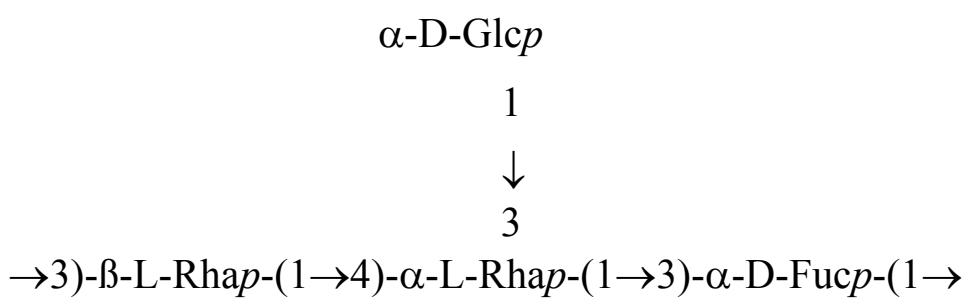

But, even more interesting, the O-chain of Xanthomonas maltophilia serogroup O19 (WINN et al., 1993) appeared to be identical with that of Erwinia carotovora ssp. carotovora, strain 436. The bacterium Xanthomonas maltophilia, earlier named Pseudomonas maltophilia (HUGH and RYSCHENKOW, 1961) was recently named Stenotrophomonas maltophilia (PALLERONI and BRADBURY, 1993). This species is not a plant pathogen and most of its strains have been isolated from clinical specimens (human faeces, blood, pericardial fluid, ascitic fluid, pleural fluid, spinal fluid, oropharyngeal swabs and contaminated tissue). However, it can be an opportunistic human pathogen, and it is also found in river water, well water, raw milk, frozen food and in the rhizosphere of some cultivated plants. The colonies formed by some Xanthomonas maltophilia (phenon 1) strains are umbonate, do not have an even colony margin and do not form slime. Xanthomonas maltophilia is a cosmopolitan in distribution and a heterogeneous collection of opportunistic bacteria (SWINGS et al., 1983).

There are some physiological similarities between Erwinia carotovora subsp. carotovora and Xanthomonas (=Stenotrophomonas) maltophilia. Thus, both species do not produce acid from glucose, indol formation is negative, the optimal temperature for growth is $35{ }^{\circ} \mathrm{C}$, and the following physiological reactions are positive: nitrate reduction, hydrolysis of gelatine, acid produced from maltose and use of cellobiose as carbon source for growth. Both bacterial species can be isolated from the rhizosphere of some cultivated plants, they are cosmopolitans and heterogeneous. Some $X$. maltophilia strains contain a respiratory nitrate reductase. It might enable cells to use nitrate as an electron sink under semi-aerobic conditions (WOODARD et al., 1990). Nevertheless, the earlier strains of X. maltophilia were strictly aerobic (HUGH and RYSCHENKOW, 1961) in contrast to E. carotovora (BRADBURY, 1986).

Fifteen distinct somatic serotypes were encounted among 26 strains of $X$. maltophilia (HUGH and RYSCHENKOW, 1961), while for E. carotovora 36 serogroups have been differentiated (DE BOER and Mc NAUGHTON, 1987). Since different serogroups have often been attributed to different LPS-structures, especially the O-chains, it can be assumed that different O-chain structures occur in both of the heterogeneous species E. carotovora and $X$. 
maltophilia. Therefore, the here reported finding of identical O-chain structures in both species may be accidentally and not reproducible for the whole spectrum of different strains occurring in both species.

\subsection{Preliminary structure of the LPS-O-chain of Erwinia carotovora ssp. atroseptica}

Interestingly, the LPS-O-chain of Eca strain 2967 was completely different from that of Ecc strain 436. It is yet unknown, whether all Eca strains have a similar structure, and what is the exact structure of the higher branched sugar. Its similarity to caryophyllose from Pseudomonas caryophilli (ADINOLFI et al., $1995 \mathrm{a}$ and b) appears interestingly. This pathogen causes bacterial wilt of carnation with similar symptoms than blackleg of potato plants, that is a sticky, brown, basal stem rot (LELLIOTT and STEAD, 1987). Future studies have to reveal to which extent different LPS-O-chains occur in the Erwinia carotovora subspecies carotovora and atroseptica.

4.3

\section{Virulence of the Japanese "rough" Eca-strain in comparison to other "smooth" Erwinia carotovora strains}

Our results clearly showed a strong difference between the two Eca strains. The German strain (with intact O-chain) was highly virulent, whereas the Japanese strain (without O-chain) was very weakly virulent on potato discs in laboratory experiments. Also, in the green house experiments potato plants developed typical blackleg symptoms by the German strain one week after inoculation, whereas the Japanese strain did not cause any symptoms one month after inoculation. Similarly, thick leaves of Chinese cabbage showed an extended soft-rotting by strain Eca 2967, but only small soft-rotted lesions by Eca 2962. Thus, the Japanese strain was weakly virulent on Chinese cabbage in spite that this strain was originally isolated from Chinese cabbage, whereas the German strain showed strong softening symptoms one day after inoculation.

The pathogenicity of $E c a$ and $E c c$ strains for Chinese cabbage has been observed by several other workers. CHIU et al. (1965) reported that Erwinia phytophthora (E. atroseptica) infected Chinese cabbage and formed a decayed area of $40 \mathrm{~mm}$ diameter within $24 \mathrm{~h}$ at $28{ }^{\circ} \mathrm{C}$ and relative humidity of $100 \%$. Also, BRADBURY (1986) reported that Ecc infects Chinese cabbage (B. chinensis) so that larger fleshy organs become softened to a pulp very quickly, and 
that Eca infects the same plant and is able to cause stalk rot of cabbage. KELLER and KNÖSEL (1980) reported that the pathogen caused about $30 \%$ losses of Chinese cabbage yield.

A different pattern of symptoms was provoked on leaves of tomato, potato, tobacco and Chinese cabbage (Figs. 4, 5, 6 and 7), where either no symptoms appeared (on tobacco and Chinese cabbage), or HR-like necrotic leaf spots occurred shortly after inoculation (on leaves of tomato and potato) (Figs. 4, 5 and 6). These symptoms should be regarded as a resistant reaction because they were only caused by high bacterial concentrations $\left(10^{8}-10^{9}\right.$ cfu/ml). MÜLLER et al. (1998) investigated the role of whole LPS as well as its subunits Ochain and core region on induced resistance in compatible and incompatible systems and found that lipid A alone can cause this effect, while the O-chain or the core region did not induce resistance. Therefore, the higher capability of the rough Eca strain (GSPB 2962) to cause necrotic spots on tomato leaves may be due to the fact that the LPS of this strain was not masked by long O-chains.

Also, other laboratories reported a lowered virulence of Erwinia carotovora strains which had defects in the LPS-O-chain. TOTH et al. (1999) found that from 40 spontaneous bacteriophage-resistant mutants of $E c a$ one mutant, A5/22, showed significantly reduced virulence in planta (blackleg symptoms) and a weaker ability to cause potato soft rot compared with the wild-type strain. On the other hand, this mutant also showed reduced synthesis of the exoenzymes pectate lyase (Pel) and cellulase (Cel) in the supernatant. However, enzyme activities were not compared with bacterial numbers, as was done in our studies. PAGE showed that the mutant strain A5/22 had a defect in the LPS-O-chain compared to the wildtype strain. SCHOONEJANS et al. (1987) used bacteriophages ФEC2 and Mu to isolate lipopolysaccharide (LPS)-defective mutants of Ech that also exhibited reduced virulence when inoculated into Saintpaulia ionantha plants. Also, PIRHONEN et al. (1991) screened for LPSdefective mutants of Ecc after transposon mutagenesis. These authors described three LPSdefective mutants which exhibited a galactose-sensitive phenotype and were impaired in virulence.

Also, for other phytopathogenic bacteria several data indicate that LPS, and especially the Ochain, contribute to bacterial virulence (NEWMANN et al., 1995), whereas the role of exopolysaccharides, for instance xanthan, in pathogenesis has not been completely unravelled. In the case of Xanthomonas campestris pv. campestris the O-chain polysaccharide (OPS) of the LPS was regarded as a factor of pathogenicity (STEINMANN, 1996). Similarly, the results obtained by LAUX (1998) imply that LPS from a smooth(s)-strain and a less virulent 
rough(r)-mutant of Pseudomonas syringae pv. phaseolicola react differently with pectins of a sensitive bush bean. Therefore, the low virulence of the rough(r)-Eca strain 2962 might be explained by its incomplete O-specific chain. However, before reaching this conclusion the question had to be answered whether strain Eca 2962 was also impaired in other factors of virulence, especially the release of extracellular enzymes.

Most strains of Erwinia carotovora produce several extracellular enzymes that degrade plant cell walls, such as pectate lyase, pectin lyase, polygalacturonase, pectin methyl esterase, cellulase and protease (PEROMBELON and KELMAN, 1980; BARRAS et al., 1994; SALMOND et al., 1994; PEROMBELON and SALMOND, 1995). These different enzymes enable the bacterium to cause tissue maceration and necrosis (COLLMER and KEEN, 1986; KOTOUJANSKY, 1987). Most of these enzymes have been shown to be important in virulence, since mutations affecting their synthesis led to reduction or loss of virulence (KOTOUJANSKY, 1987). Thus, mutations that affect synthesis or secretion of extracellular enzymes from Erwinia spp. (Eca, Ecc and Ech) led to a reduction or loss of virulence in planta (MURATA et al., 1990; REEVES et al., 1993). Therefore, we determined the pectinase activity of the two Eca-strains 2962 and 2967. According to our results as shown in Tables 2 and 3 it might be concluded that the Japanese strain (GSPB 2962) possesses a lower pectinase activity than the German strain (GSPB 2967) when determined in the supernatant. However, when the pectinase activity was calculated per bacterial cell in vitro both strains showed the same pectinase activity. We concluded from these results that the weak virulence of the Japanese strain can not be attributed to a lower pectinase activity.

On the other hand, the slow growth of the Japanese strain in the liquid pectin medium might be explained by the low pectinase activity of this strain. This possibility was excluded by our finding that in King's medium B (without pectin) the Japanese strain also grew much slower than the smooth and virulent strain 2967, the difference being even higher than in liquid medium. Obviously, the Japanese strain (2962) possessed a lower growth vigour than the virulent Eca strain. In most other cases of phytopathogenic bacteria the rough variants grow even faster or as fast as the smooth wild strains, for instance in case of Ralstonia solanacearum (HUSSAIN and KELMAN, 1958) or P. syringae pv. phaseolicola (GERWE et al., 1987). Therefore, it appears not very probable that the low growth vigour of strain 2962 was related to the loss of the LPS-O-chain.

However, dependencies between growth, nutrient composition and LPS production have been reported for E. carotovora. The results obtained by FUKUOKA et al. (1989) show that the combination of two types of carbon sources enhanced LPS productivity, cell growth, and 
furthermore, productivity per unit cell. When E. carotovora was cultivated on a basal medium or the medium containing a single carbon source, the LPS yield was poor.

Since the reason for the low growth vigour of the Japanese strain is completely unknown it is still impossible to answer the question whether the loss of the LPS-O-chain, or the low growth vigour, or both deficiencies are responsible for the low virulence of the Japanese Eca strain (GSPB 2962). 
1- Electrophoresis of the LPS of the Japanese Eca strain (2962), originally isolated from Chinese cabbage, revealed an absence of the O-chain, that is a "rough" LPS. In contrast, the German strains of Eca (2967) and Ecc (436) all contained "smooth" LPS with an O-chain, indicated by the typical ladder-like pattern after electrophoresis.

2- The repeating unit of the O-chain of Ecc 436 was elucidated as a tetrasaccharide of the following structure:

$$
\begin{gathered}
\rightarrow 3)- \text {-L-Rhap- }(1 \rightarrow 4)-\alpha-\mathrm{L}-\mathrm{Rha} p-(1 \rightarrow 3)-\alpha-\mathrm{D}-\mathrm{Fuc} p-(1 \rightarrow \\
3 \\
\uparrow \\
1 \\
\alpha-\mathrm{D}-\mathrm{Glc} p
\end{gathered}
$$

3- The repeating unit of the O-chain of Eca 2967 was identified as a pentasaccharide containing three galactose residues, one rhamnose and a new higher branched sugar (similar to caryophyllose).

4- The Japanese Eca-strain (2962) was very weakly virulent, as indicated by a weak soft rotting of potato slices and Chinese cabbage leaves and the inability to cause blackleg symptoms of potato plants, in contrast to the highly virulent Eca-strain 2967.

5- When inoculated into young leaves at high concentrations $\left(10^{8}\right.$ to $\left.10^{9} \mathrm{cfu} / \mathrm{ml}\right)$, the Japanese Eca strain (2962) caused a little bit stronger necrotic leaf spots and chlorosis on tomato compared to those by the German Eca strain (2967), whereas similar symptoms were caused by both strains on potato leaves, and no symptoms were detectable on leaves of tobacco and Chinese cabbage.

6- The Japanese strain Eca (2962) produced lower pectinolytic activity in the supernatant of in vitro cultures than the German Eca strain (2967). However, when the pectinolytic activities were calculated per bacterial cell, the enzyme activities did not differ significantly between the two strains.

7- However, the German Eca strain (2967) showed a much higher growth vigour in liquid pectin medium as well as in King's medium B than the Japanese strain 2962.

8- Similarly, the German Eca-strain grew faster and produced deeper cavities on the CVPM medium than the Japanese strain.

9- The results obtained clearly showed that the Japanese strain had a slower growth vigour on any artificial medium, as well as a lower disease causing capability on different plants. 
Whether this defect was caused by the confirmed loss of the O-specific LPS-chain is unknown but does not appear very probable. 


\section{CHAPTER IV}

\section{Detection of Erwinia carotovora subsp. atroseptica and Erwinia carotovora subsp. carotovora by Polymerase Chain Reaction (PCR)}

\section{INTRODUCTION}

Under temperate conditions, soft rot of potato crops is primarily caused by two of the five subspecies recognized within Erwinia carotovora. While E. carotovora ssp. carotovora is widespread and has a wide host range besides potato, E. carotovora ssp. atroseptica is more specifically associated with potato and is prevalent under cold to temperate climates. Both subspecies are also responsible for severe losses in the field (HELIAS et al., 1998). Often, the bacteria occur as latent contamination on potato tubers, and the actual occurrence of soft rot depends on environmental factors (PEROMBELON and KELMAN, 1980).

Different methods to assess microbial contamination of potato tubers have been the subject of various studies. Classical methods based on biochemical and biological tests of the isolated bacteria on selective media have proved to be useful tools for identification and enumeration of soft rot Erwinia spp. (STEWART, 1962; CUPPLES and KELMAN, 1974; BDLIYA, 1995) but are laborious and time consuming (SMID et al., 1995). Therefore, serological and more recently molecular methods, such as the polymerase chain reaction (PCR), have been developed to detect and characterize Erwinia carotovora. However, not all of these methods can be used routinely for analyses of potato tubers, or allow specific detection of each Erwinia spp. (HELIAS et al., 1998). Serological methods are one way of detecting erwinias on potato tubers (De BOER and McNAUGHTON, 1987), but the serological diversity of strains in some countries do not allow reliable detection by these methods (KANKILA, 1989).

Most recently, the polymerase chain reaction (PCR) was proposed for the detection of tuber contamination by Erwinia spp. (ANONYMOUS, 1992). In the PCR a target DNA fragment is amplified enzymatically. Thus, PCR combines a high degree of specificity with a high degree of sensitivity. PCR technology has been successfully developed as a tool for specific and sensitive detection of micro-organisms in clinical (HARTSKEER et al., 1989) and environmental samples (BEI et al., 1991; ATLAS et al., 1992).

When detecting plant pathogenic microorganisms in infested potato tubers by means of PCR, there is always the problem of high amounts of plant substances, such as starch and phenolic compounds, being coextracted, interferring with or even inhibiting the enzyme Taqpolymerase necessary for amplifying the target DNA in the PCR reaction (NIEPOLD, 1994; 
VAN DER WOLF et al., 1996; HYMAN et al., 1997). Recently, several DNA primers shown to be specific for Eca or Ecc regardless of serogroup, have been generated and used in PCRbased assays (DE BOER and WARD 1995; FRECHON et al., 1995; SMID et al., 1995).

The purpose of this study was to test the feasiblity, reliability and sensitivity of the PCR for assessing the latent contamination of potato tubers by erwinias. For this aim two primer sets were compared to assess and evaluate the best and most efficient method for detecting Erwinia spp.. 


\section{MATERIALS AND METHODS}

\subsection{Solutions and buffers for agarose gel electrophoresis}

$10 \mathrm{x}$-Electrophoresis buffer (tris-boric acid-EDTA (TBE), $1000 \mathrm{ml}$ ) (SAMBROOK et al., 1989)

Tris

$0.89 \mathrm{M}$

Boric acid

$0.89 \mathrm{M}$

$\mathrm{Na}_{2}$ EDTA X $2 \mathrm{H}_{2} \mathrm{O}$

$0.02 \mathrm{M}$

107.8 $\mathrm{g}$ Tris, $55.0 \mathrm{~g}$ boric acid and $7.44 \mathrm{~g} \mathrm{Na}_{2}$ EDTA were dissolved in $1000 \mathrm{ml}$ distilled water and stored at room temperature (stock solution). For electrophoresis, the solution was diluted 1:10 with distilled water.

\section{5 x- Loading buffer $(10 \mathrm{ml})$}

Glycerol

TBE

Bromophenol blue (BPB)
$50 \%$

$5 \mathrm{x}$ half strength of stock solution

$0.3 \%$

$30 \mathrm{mg}$ BPB were dissolved in $4.25 \mathrm{ml}$ of $10 \mathrm{x}$-TBE and $5.75 \mathrm{ml}$ of $87 \%$ glycerol were added. The solution was stored at room temperature.

\subsection{Amplification by PCR}

The PCR procedure was carried out according to the methods described by NIEPOLD (1994) DE BOER and WARD (1995). The bacterial strains (see 2.4) were grown on King's medium B agar plates and incubated at $27^{\circ} \mathrm{C}$ for $24 \mathrm{~h}$. Bacterial suspensions were washed off the agar plates, adjusted to an $\mathrm{OD}_{660}$ of 0.06 (ca. $1.5 \times 10^{7} \mathrm{cfu} / \mathrm{ml}$ ) and ten-fold dilutions were prepared in sterile distilled water. Detection of Erwinia carotovora in each dilution was carried out by serial dilution and plating on King's medium B as well as by PCR.

For PCR, the bacterial suspension was boiled at $100{ }^{\circ} \mathrm{C}$ for $5 \mathrm{~min}$ and stored at $-20{ }^{\circ} \mathrm{C}$ until use. To reduce the risk of contamination, all materials used were sterilised. Sterile pipette tips and microvials from "biozym Diagnostic GmbH" (Hameln, Germany) were used throughout. The Erwinia carotovora primers Y1/Y2 (NIEPOLD, 1994) and Eca1f/Eca2r (DE BOER and WARD, 1995) were generated by NIPAS, Göttingen. 
PCR amplification of DNA was achieved by using two specific primers as follows: primer Y1 (5'GGACGCCGAGCTGTGGGGT3') together with primer Y2 (5'CAGGAAGATGT CGTTATCGCGAGT3') (NIEPOLD, 1994) and primers Ecalf (5'CGGCATCATA AAAACACG3') together with Eca2r (5'GCACACTTCATCCAGCGA3') (WARD \& DE BOER, 1995). A negative control was enclosed by running the PCR reaction with sterile water and the positive control was used as bacterial DNA template. The enzyme Taq polymerase (5 $\mathrm{U} / \mu \mathrm{l})$ was added directly to the reaction mix. Each PCR reaction containing a final volume of $50 \mu 1$ was prepared as described in Table 1 and covered with $50 \mu 1$ sterile mineral oil (Sigma, Deisenheim).

The PCR was performed by heating in a thermal cycle (Hybaid-Omni Gene, Heidelberg). PCR ampilification by two primers was achieved by three stages. The first stage was the initial cycle of denaturation at $95{ }^{\circ} \mathrm{C}$ for $1 \mathrm{~min}$. The second stage was performed by running fourty cycles of a denaturating temperature at $94{ }^{\circ} \mathrm{C}$ for $1 \mathrm{~min}$, annealing temperature of $65^{\circ} \mathrm{C}$ for $1 \mathrm{~min}$ and an extension phase of $72{ }^{\circ} \mathrm{C}$ for $45 \mathrm{sec}$. The same temperature was used for both primer pairs. The third stage, an 8 min extension period, was added after the final cycle.

\subsection{Analysis of the PCR product using agarose gel electrophoresis}

Gel electrophoretic analysis was performed by using 1.5\% agarose NEEO Ultra-Quality gels (Roth, Karlsruhe) in $1 \times$ TBE separation buffer, pH 8.0. The gel electrophoresis apparatus used was a horizontal gel electrophoresis GNA-100 and GNA-200 (Pharmacia, Freiburg). Agarose was weighed, suspended in buffer and melted in a microwave until a transparent solution was achieved. After cooling until about $45-50{ }^{\circ} \mathrm{C}$, the agarose was poured into a plastic mold and allowed to solidify at room temperature. Air bubbles were removed with a pipette tip direct after pouring. After the gel was completely polymerized, it was tranferred to a gel tank containing the electrophoresis running buffer $(1 \times \mathrm{TBE}, \mathrm{pH}$ 8.0) and the same buffer was added just enough to cover the top of the gel to a depth of 1-2 mm (DARLING and BRICKELL, 1994).

The samples were prepared as follows: Two $\mu l$ of the loading buffer was pipetted onto a para film strip equal to the number of the performed PCR reactions and $8 \mu \mathrm{l}$ from each of the PCR reaction were pipetted out by mixing with the loading buffer on the parafilm strip. The whole volume was transferred into the slots of the agarose gel. The lid of the electrophoresis tank was closed and the electrical leads connected to the power pack (H. Hölzer, Dorfen). A voltage of 5 $\mathrm{V} / \mathrm{cm}$ was set, and the sample migration from the anode to the cathode was visualised by the migration of the $5 \mathrm{x}$ Loading Dye solution along the gel, that was allowed to run for 30-60 min 
at room temperature. After DNA fragments were separated (in the electric field), the gel was removed carefully and put in $0.5 \mu \mathrm{g} / \mathrm{ml}$ ethidium bromide (Fluka, New-Ulm) solution for 10 min. The gel was removed again and submersed in distilled water for $5 \mathrm{~min}$.

The gel was photographed with a Polariod MP4 camera provided with a red filter at $590 \mathrm{~nm}$, using a black and white coaterless film polapan 667 (Polariod, St. Albans, Hertfordshirre, UK) under UV apparatus Intas $254 \mathrm{~nm}$ designed by Polariod (Intas, Göttingen). The gel was also visualised under UV transilluminator (Bio-Rad Gel Doc 1000, Munich), and saved as data by Multi Analyst program. The expected size of the PCR product was 430 bp for primers Y1 \&Y2 and $690 \mathrm{bp}$ for primers Eca1f and Eca2r, respectively.

Table 1: Reaction mix for PCR reagents used for DNA amplification

\begin{tabular}{|l|l|}
\hline Reagents & Concentration per reaction \\
\hline Dist. water (sterile) to final volume & $50 \mu \mathrm{l}$ \\
\hline $10 \mathrm{x}$ PCR buffer & $1 \%(\mathrm{v} / \mathrm{v})$ \\
\hline DNTP (Fermentas, St. Leon-Rot) & $0.2 \mathrm{mM}$ \\
\hline Each primer solution (Y1 \& Y2) & $1 \mu \mathrm{M}$ \\
\hline Each primer solution (ECAIf \& ECA2r) & $1 \mu \mathrm{M}$ \\
\hline Tween 20 & $10 \%(\mathrm{v} / \mathrm{v})$ \\
\hline Taq-DNA polymerase & $1.0 \mathrm{U}$ \\
\hline Bacterial template & $3 \mu \mathrm{l}$ \\
\hline
\end{tabular}

\subsection{Determination of the detection limit of the PCR method}

The bacterial strains were grown on King's medium B plates at $27{ }^{\circ} \mathrm{C}$ for $24 \mathrm{~h}$. For this experiment the bacterial strains were adjusted to an $\mathrm{OD}_{660}$ of $0.06\left(\mathrm{ca} .10^{8} \mathrm{cfu} / \mathrm{ml}\right)$ in sterile distilled water and then boiled at $100{ }^{\circ} \mathrm{C}$ for $5 \mathrm{~min}$. From each dilution $3 \mu \mathrm{l}$ were added to the PCR reaction mixture and analysed following the procedure described in 2.2.

\subsection{Determination of Eca and Ecc with specific primers}

Different bacterial strains of $E c a$ and $E c c$, five $E c a$ (GSPB nos. 2962, 9201, 2967,1405 and 2231) and four Ecc (GSPB nos. 426, 429, 436 and 1405) were grown as described in 2.2. Each 
bacterial strain was adjusted to an $\mathrm{OD}_{660}$ of $0.06\left(\mathrm{ca} .10^{8} \mathrm{cfu} / \mathrm{ml}\right)$ in sterile distilled water and then boiled at $100{ }^{\circ} \mathrm{C}$ for $5 \mathrm{~min}$ and analysed following the procedure as described in 2.2 .

\subsection{Effect of potato peel extract on the detection limit of Eca by PCR}

Four methods of DNA extraction from potato peel extract artificially contaminated by Erwinia carotovora ssp. atroseptica or carotovora were compared as follows:

\subsubsection{DNA isolation by using the method of LI and DE BOER (1995)}

Potato tubers were peeled (ca. $1 \mathrm{~g}$ peel) with a knife hand peeler and crushed in a sterile mortar with $4 \mathrm{ml}$ TE buffer $\mathrm{pH} 8.0$ (10 mM Tris-HCl, $\mathrm{pH} 8.0,1 \mathrm{mM}$ EDTA), and $0.1 \mathrm{ml}$ of bacterial suspension (ca. $1.8 \times 10^{8} \mathrm{cfu} / \mathrm{ml}$ ) was inoculated into the homogenate and serially diluted. Aliquots $(100 \mu \mathrm{l})$ from the plant homogenate were centrifuged at $8.000 \mathrm{~g}$ for $10 \mathrm{~min}$ at $4{ }^{\circ} \mathrm{C}$ in Eppendorf tubes. The PCR protocol steps for DNA purification were applied as follows:

A - The pellet was frozen at $-20{ }^{\circ} \mathrm{C}$ for $1 \mathrm{~h}$, thawed at room temperature for $10 \mathrm{~min}$, mixed with $100 \mu \mathrm{l}$ cold acetone $\left(-20^{\circ} \mathrm{C}\right)$ and incubated for $10 \mathrm{~min}$, suspended in $500 \mu \mathrm{l}$ of TE buffer, followed by addition of $50 \mu \mathrm{l}$ of $250 \mathrm{mM}$ EDTA, $\mathrm{pH} 8.0,50 \mu 1$ of $14 \%$ SDS, and $10 \mu 1$ proteinase K (Merck, Darmstadt) and incubated for $1 \mathrm{~h}$ at $55^{\circ} \mathrm{C}$.

$\mathrm{B}$ - An equal volume of 7.5 $\mathrm{M}$ ammonium acetate was added to separate most the cell debris from the DNA by pelleting the precipitate in a centrifuge at $14.000 \mathrm{~g}$ for $20 \mathrm{~min}$ at $4{ }^{\circ} \mathrm{C}$.

$\mathrm{C}$ - The clear supernatant $(0.8 \mathrm{ml})$ was transferred to a new tube, and 0.8 volume isopropanol $\left(-20^{\circ} \mathrm{C}\right)$ were added and incubated for $30 \mathrm{~min}$ at $-20^{\circ} \mathrm{C}$.

$\mathrm{D}$ - The DNA was pelleted by centrifugation at $14.000 \mathrm{~g}$ for $20 \mathrm{~min}$ at $4{ }^{\circ} \mathrm{C}$. The pellet was washed with $70 \%$ ethanol and precipitated by a second centrifugation at $14.000 \mathrm{~g}$ for $20 \mathrm{~min}$ at $4{ }^{\circ} \mathrm{C}$.

E - After decanting the supernatant, the DNA pellet was dried in the tube at room temperature for $0.5 \mathrm{~h}$, and finally the DNA was resuspended in $50 \mu 1 \mathrm{TE}$ buffer ( $\mathrm{pH} 8.0)$ and incubated at 4 ${ }^{\circ} \mathrm{C}$ overnight. All samples were stored at $-20{ }^{\circ} \mathrm{C}$. For the PCR procedure $2 \mu 1$ of each DNA sample were used.

\subsubsection{DNA isolation by using the method of MÖLLER et al. (1992)}

1- Peel $0.5 \mathrm{~g}$ potato tuber with a knife hand peeler and grind the peel with a sterile mortar in liquid nitrogen. 
2- Mix the homogenate with $2 \mathrm{ml}$ TES buffer (0.1 $\mathrm{mM}$ Tris pH 8.0, $10 \mathrm{mM}$ EDTA, 2\% SDS), and inoculate $0.1 \mathrm{ml}$ from a bacterial suspension (ca. $10^{8} \mathrm{cfu} / \mathrm{ml}$ ) to the homogenate, which is then serially diluted.

3- $500 \mu 1$ are taken from the homogenate and $5 \mu 1$ proteinase K (Merck, Darmstadt) are added to each E-cup tube and incubated at $55-60{ }^{\circ} \mathrm{C}$ for $1 \mathrm{~h}$.

4- Add $140 \mu 15 \mathrm{M} \mathrm{NaCl}$ and $65 \mu 1$ 10\% CTAB into each Eppendorf tube and incubate at $65^{\circ} \mathrm{C}$ for $10 \mathrm{~min}$.

5- Add 1 volume $(700 \mu \mathrm{l})$ of chloroform/isoamylalcohol = 24:1 (v/v), mix gently, place on ice for $30 \mathrm{~min}$, centrifuge at $12.000 \mathrm{~g}$ for $10 \mathrm{~min}$ at $4{ }^{\circ} \mathrm{C}$.

6- Transfer the supernatant to a fresh tube, add $225 \mu 15 \mathrm{M} \mathrm{NH}_{4}$ acetate, mix gently, place on ice for $30 \mathrm{~min}$, centrifuge at $12.000 \mathrm{~g}$ for $5 \mathrm{~min}$ at $4{ }^{\circ} \mathrm{C}$.

7- Transfer the supernatant to a fresh tube, add 0.55 vol $(510 \mu \mathrm{l})$ to precipitate DNA, centrifuge immediately for $5 \mathrm{~min}$ at $12.000 \mathrm{~g}$ for $5 \mathrm{~min}$ at $4{ }^{\circ} \mathrm{C}$.

8- Decant the supernatant and wash the pellet twice with cold $70 \%$ ethanol, dry the pellet at room temperature and dissolve the pellet in $50 \mu 1 \mathrm{TE}$ buffer $\mathrm{pH}$ 8.0.

\subsubsection{DNA isolation by using the NaOH method (NIEPOLD, 1999a)}

Potato tubers were peeled with a knife hand peeler and crushed in a sterile mortar with $4 \mathrm{ml}$ sterile water, and $0.1 \mathrm{ml}$ from a bacterial suspension (ca. $10^{8} \mathrm{cfu} / \mathrm{ml}$ ) were inoculated into the homogenate and serially diluted. DNA was extracted a shown on the following flow chart:

$1 \mathrm{ml}$ potato tuber extract

$10 \mu 1$ lysozyme $(20 \mathrm{mg} / \mathrm{ml})$

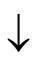

$10 \mu l$ proteinase $\mathrm{K}(20 \mathrm{mg} / \mathrm{ml})$

Incubation for $10 \mathrm{~min}$ at $37^{\circ} \mathrm{C}$

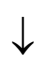

$10 \mu \mathrm{l}$ of the lysed sample are mixed with $20 \mu 10.5 \mathrm{M} \mathrm{NaOH}$

incubation for $10 \mathrm{~min}$ at $95^{\circ} \mathrm{C}$

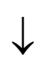

Chilling on ice for 10-30 min

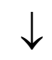


Transfer of $5 \mu 1$ of the lysate into a new tube

Add 20-45 $\mu 1$ Tris/HCl/BSA (3\%) buffer ( $\mathrm{pH} 7.5$ ) to the $5 \mu$ lysate sample for extraction of a low starch potato variety, mix and incubate at room temperature for $5 \min$ (1:5-10 dilution), 45 $\mu \mathrm{l}$ for extraction of a high starch potato variety, mix and incubate at room temperature for 5 $\min (1: 5-10$ dilution).

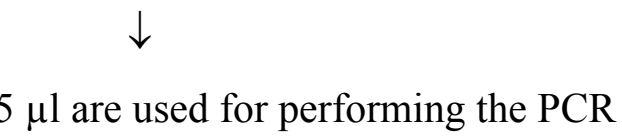

\subsubsection{DNA isolation by using the method of the German Plant Protection Service, Hannover (PASTRIK and RAINEY, 1999)}

1- Potato tubers are peeled with a knife hand peeler and crushed in a sterile mortar with $4 \mathrm{ml}$ sterile water, and $0.1 \mathrm{ml}$ of bacterial suspension (ca. $10^{8} \mathrm{cfu} / \mathrm{ml}$ ) is inoculated into the homogenate and serially diluted. The plant homogenate aliquots $(100 \mu l)$ are centrifuged at $8.000 \mathrm{~g}$ for $10 \mathrm{~min}$ at $4{ }^{\circ} \mathrm{C}$ in Eppendorf tubes.

2- After decanting, the pellet is resuspended in $220 \mu$ Lysis-buffer $(100 \mathrm{mM} \mathrm{NaCl}, 10 \mathrm{mM}$ Tris-HCl, $\mathrm{pH} 8.0$ and $1 \mathrm{mM}$ EDTA, $\mathrm{pH} 8.0$ ) in a $1.5 \mathrm{ml}$ Eppendorf tube.

3- Tubes are kept for $10 \mathrm{~min}$ at $95{ }^{\circ} \mathrm{C}$ in a heating block and the samples put on ice for $5 \mathrm{~min}$.

4- Add $80 \mu \mathrm{l}$ of lysozyme stock solution ( $50 \mathrm{mg} / \mathrm{ml}$ lysozyme in $10 \mathrm{mM}$ Tris-HCL, $\mathrm{pH} 8.0$ ) and incubate at $37^{\circ} \mathrm{C}$ for $30 \mathrm{~min}$.

5- $220 \mu \mathrm{l}$ of solution A (Invitogen BV, Netherland) are added, mixed well and incubated at 65 ${ }^{\circ} \mathrm{C}$ for $30 \mathrm{~min}$.

6- $100 \mu \mathrm{l}$ of solution B (Invitogen BV, Netherland) are added and mixed by vortexing until the precipitate moves freely in the tube and the sample is uniformly viscous.

7- $500 \mu \mathrm{l}$ chloroform are added and vortexed until viscosity decreased and the mix is homogeneous.

8- The samples are centrifugated at $15.000 \mathrm{~g}$ for $20 \mathrm{~min}$ at $4{ }^{\circ} \mathrm{C}$ to separate the phases forming an interphase, and the upper phase is transferred into a fresh microcentrifuge tube.

9- $1 \mathrm{ml}$ ethanol $100 \%\left(-20^{\circ} \mathrm{C}\right)$ is added, vortexed briefly and incubated on ice for $10 \mathrm{~min}$.

10 - The pellet is obtained by centrifugation at $15.000 \mathrm{~g}$ for $20 \mathrm{~min}$ at $4{ }^{\circ} \mathrm{C}$.

11- The pellet is washed with $500 \mu \mathrm{l}$ ethanol $80 \%\left(-20^{\circ} \mathrm{C}\right)$, mixed by inverting the tube, and again centrifuged at $15.000 \mathrm{~g}$ for $20 \mathrm{~min}$ at $4{ }^{\circ} \mathrm{C}$. 
12- The pellet is dried by decanting the supernatant and remaining the tubes openly at room temperature for $0.5 \mathrm{~h}$. Finally the pellet is resuspended in $100 \mu \mathrm{l}$ sterile $\mathrm{H}_{2} \mathrm{O}$, and $2 \mu \mathrm{l}$ of the DNA extract are used in the PCR. 


\section{$3 \quad$ RESULTS}

\subsection{Detection and identification of Erwinia spp. using the polymerase reaction}

The first step in this study was to evaluate the specificity of the two Erwinia spp. primer pairs Y1\&Y2 and Eca1f \& Eca2r. For comparison 9 bacterial strains, 5 from Eca and and 4 from $E c c$, were tested. For extracting the DNA, the bacteria were boiled in water (see 2.3). The results obtained show that all bacterial strains from $E c a$ and Ecc yielded a distinct band of 434 bp in size with primers Y1 and Y2 (Fig. 1 a) while only Eca strains showed a signal product of 690 bp using the primers Eca1f \& Eca2r (Fig.1 b). It can be concluded from these results that the specificity of primers Y1 and Y2 was broader because they could detect $E c a$ and Ecc equally well, whereas primers Eca1f \& Eca2r detected only Eca.

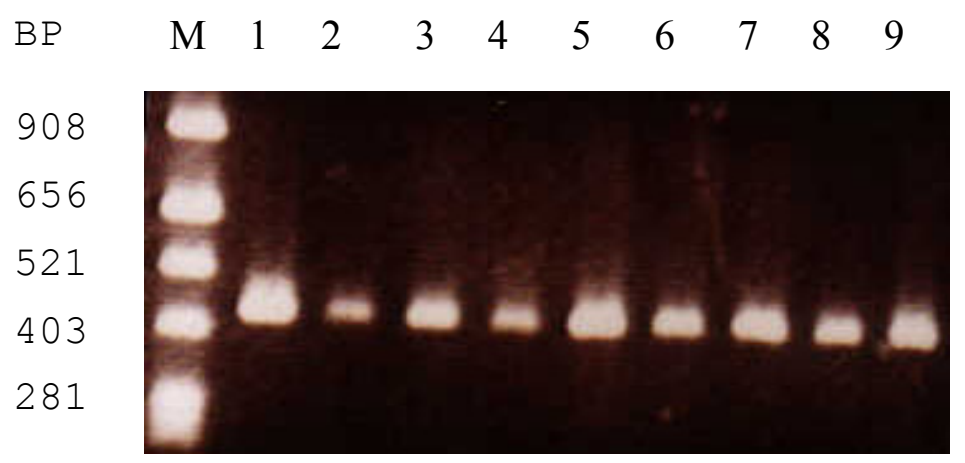

Fig. 1a: Reaction of $E c a$ and $E c c$ strains using primers $\mathrm{Y} 1 \& \mathrm{Y} 2$. The PCR product after 40 cycles was separated by agarose gel electrophoresis. Lanes: (M) marker pBR/ AIu 20, (1) Eca GSPB 2231, (2) Eca GSPB 1401, (3) Eca GSPB 2967, (4) Eca GSPB 2962, (5) Eca GSPB 9201, lanes: (6) Ecc GSPB 436, (7) Ecc GSPB 426, (8) Ecc GSPB 1405, and (9) Ecc GSPB 429. 


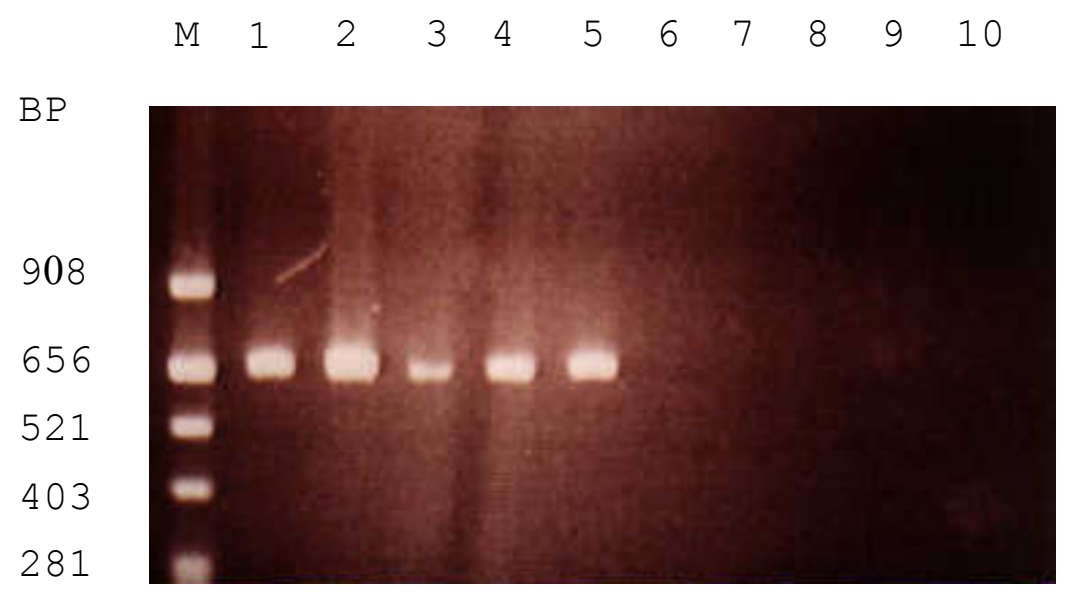

Fig. 1b: Reaction of Eca and Ecc strains using primers Eca1f \& Eca2r. The PCR product after 40 cycles was separated by agarose gel electrophoresis. Lanes: (M) marker pBR/ AIu 20, (1) Eca GSPB 2231, (2) Eca GSPB 1401, (3) Eca GSPB 2967, (4) Eca GSPB 2962, (5) Eca GSPB 9201, lanes: (6) Ecc GSPB 436, (7) Ecc GSPB 426, (8) Ecc GSPB 1405, Ecc GSPB 427 and (9) Ecc GSPB 429.

\subsection{The detection limit of the PCR method using primers Y1 and}

\section{Y2}

For determining the detection limit of Erwinia carotovora ssp. atroseptica from bacterial cultures, it was not necessary to extract DNA from the bacteria. The method was performed by boiling the bacterial samples $(100 \mu \mathrm{l})$ from each dilution to $100{ }^{\circ} \mathrm{C}$ for $5 \mathrm{~min}$. The sensitivity of PCR technique was determined by assaying 10-fold serial dilutions of a bacterial suspension from Eca (GSPB no. 2967) as well as by determining the bacterial concentrations by plating serial dilutions on King's medium B. The results in Fig. 2 clearly show that according to the microbiological determination (plating) of bacterial concentration the detection limit of the PCR method using the Eca primers Y1 and Y2 was $1.5 \times 10^{3} \mathrm{cfu} / \mathrm{ml}$. 
Bacterial concentration $\mathrm{cfu} / \mathrm{ml}$

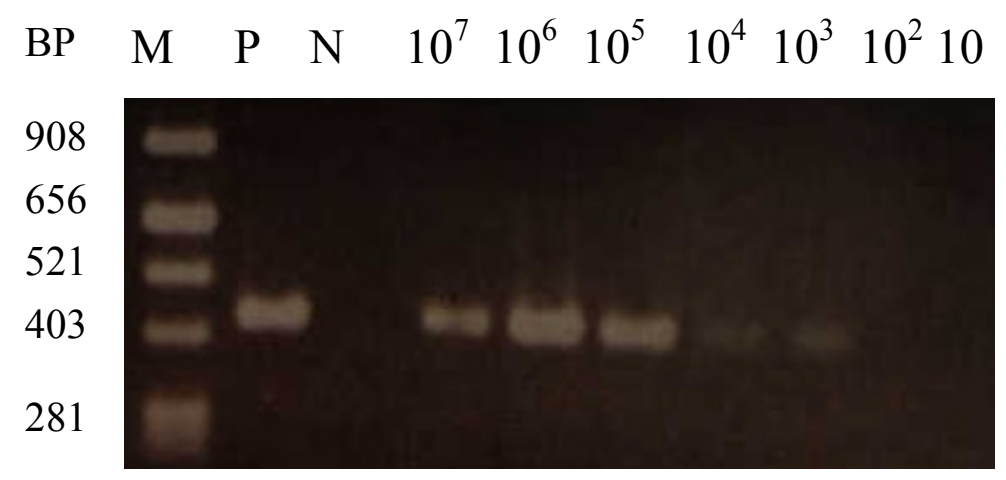

Fig. 2: Determination of the detection limit of the PCR using primers Y1 and Y2. The test was performed with a pure culture of Eca GSPB 2967. Ten-fold dilutions were prepared in sterile distilled water and boiled at $100{ }^{\circ} \mathrm{C}$ for $5 \mathrm{~min}$. The PCR product after 40 cycles was separated on agarose by electrophoresis. $\mathrm{M}=$ Marker, $\mathrm{P}=$ positive control, $\mathrm{N}=$ negative control.

\subsection{The detection limit of the PCR method using primers Ecalf and Eca2r}

In a standard PCR protocol performed with primers Eca1f and Eca2r and using a pure culture of Eca as DNA template a 690 bp product was obtained (Fig. 3). The results show that the detection limit of the PCR using the primers Eca1f and Eca2r was $1.5 \times 10^{3} \mathrm{cfu} / \mathrm{ml}$.

Bacterial concentration $\mathrm{cfu} / \mathrm{ml}$

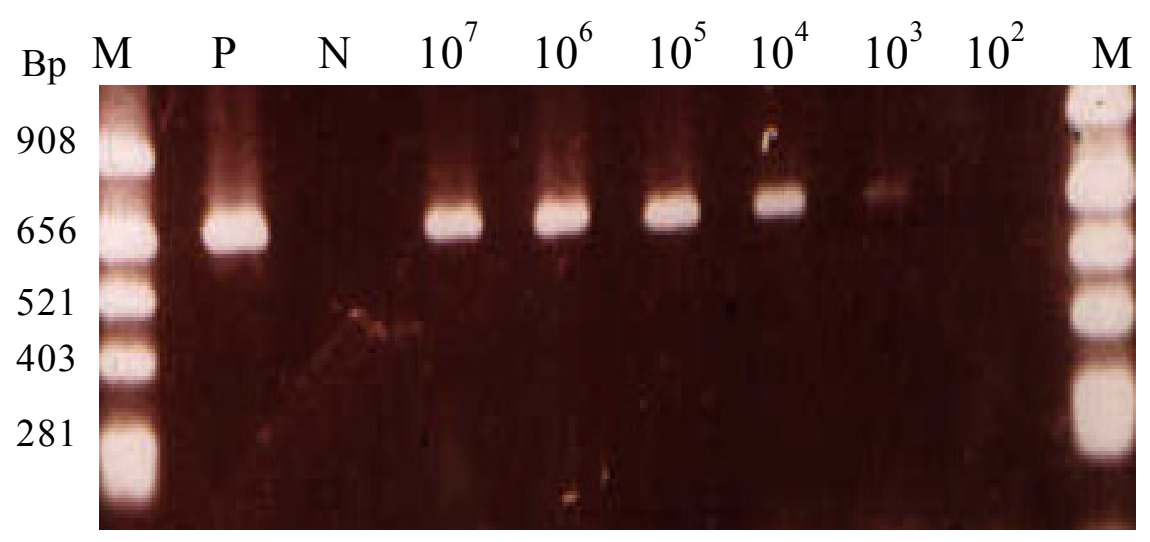

Fig. 3: Determination of the detection limit of the PCR using primers Ecalf and Eca2r. The test was performed with a pure culture of Eca GSPB 2967. Ten-fold dilutions were prepared in sterile distilled water and boiled at $100{ }^{\circ} \mathrm{C}$ for $5 \mathrm{~min}$. The PCR product after 40 cycles was separated on agarose by electrophoresis. $\mathrm{M}=$ Marker, $\mathrm{P}=$ positive control, $\mathrm{N}=$ negative control. 


\subsection{Detection of $E c a$ in artificially contaminated potato peel extract by PCR with primers Y1 and Y2}

Isolation of DNA from artificially contaminated potato peel extract was achieved by different protocols (see subchapter 2.5) for detecting soft rot bacteria from peel extracts. PCR amplification with primers Y1 and Y2 yielded a product of 434 bp only by the method of LI an DE BOER (1995) which, thus, allowed to detect Erwinia carotovora in the peel extract. By the other protocols a DNA amplification was not successful. The results as shown in Fig. 4 revealed bands only after the third sample dilution, whereas from peel extracts containing high bacterial concentrations a positive signal was not detectable. This discrepancy can be attributed to substances from the potato peel extract that inhibited the PCR reaction. The detection limit was $\mathbf{1 0}^{\mathbf{2}} \mathbf{c f u} / \mathbf{m l}$.

Sample dilution

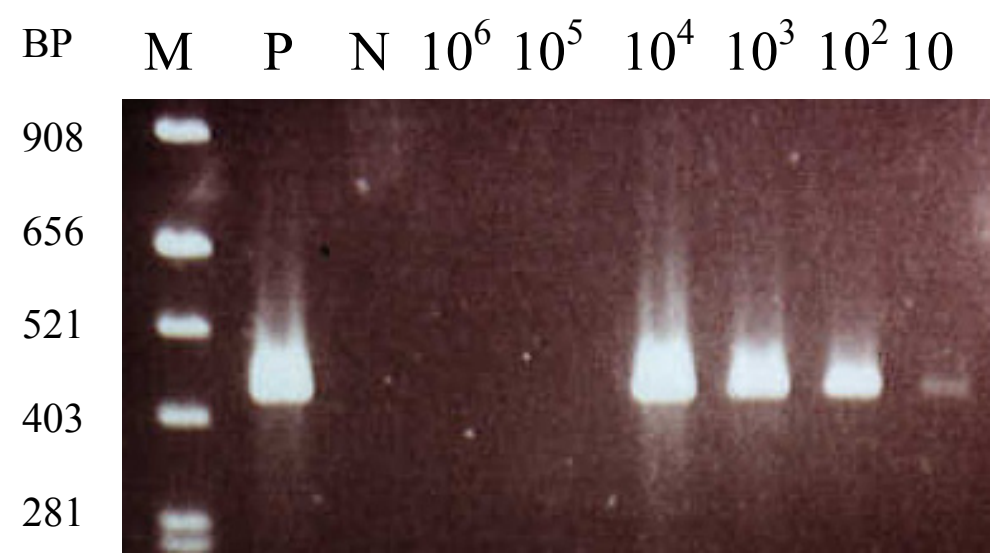

Fig. 4: Determination of $E c a$ in artificially inoculated potato peel extract by PCR using Eca primers $\mathrm{Y} 1$ and Y2. The test was carried out with ten-fold dilutions of the inoculated tuber peels. The PCR product amplified after 40 cycles was separated on agarose gel by electrophoresis. $\mathrm{M}=$ Marker, $\mathrm{P}=$ positive control, $\mathrm{N}=$ negative control.

\subsection{Detection of $E c a$ in artificially contaminated potato peel extract by PCR with primers Eca1f and Eca2r}

Fig. 5 shows a distinct signal after the second sample dilution, representation a sensitivity of the PCR of $10^{2}$ cells $/ \mathbf{m l}$. 
Sample dilution

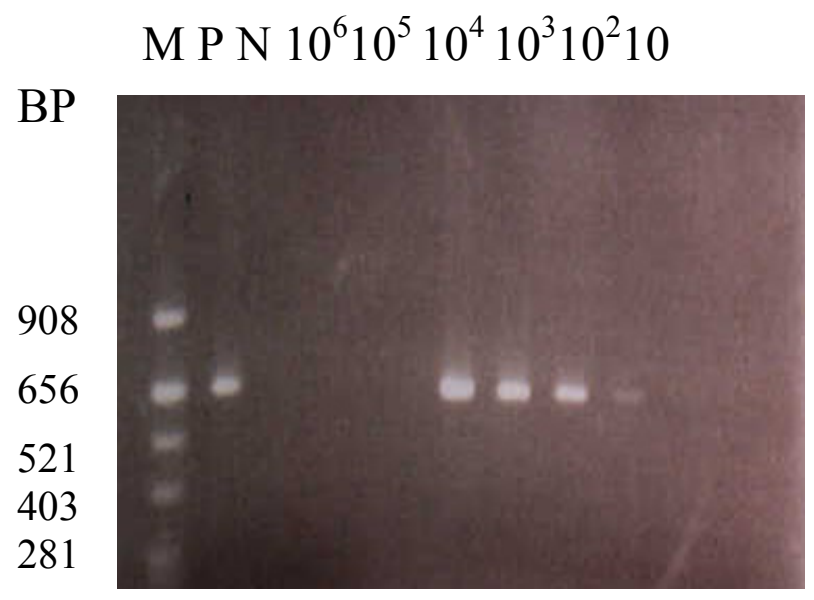

Fig. 5: Determination of Eca in artificially inoculated potato peel extracts by PCR with the Eca primers Eca1f and Eca2r. The test was carried out with ten-fold dilutions of the inoculated potato peel extracts. The PCR product amplified after 40 cycles was separated on agarose gel by electrophoresis. $\mathrm{M}=$ Marker, $\mathrm{P}=$ positive control, $\mathrm{N}=$ negative control. 
The polymerase reaction (PCR) which allows a target DNA fragment to be amplified enzymatically has been applied successfully for detecting latent contamination by Erwinia spp.. The method is based on the use of Eca-specific DNA primers to amplify a targeted Eca DNA fragment to faciliate detection of both living and dead cells (HENSEN and FRENCH, 1993). Classical techniques of bacterial isolation and characterization are time consuming and relatively insensitive because of the high level of saprophytes occurring in potato samples (LELLIOTT and STEAD, 1987). Therefore, several alternative methods have been developed for detecting Erwinia carotovora in potato tubers. These involve semi-selective media (STEWART, 1962; CUPPLES and KELMAN, 1974; BYDLIA, 1995 and AHMED et al., 2000), serological methods, such as DAS-ELISA (PEROMBELON and HYMAN, 1995; FRAAIJE et al., 1997) and IFC (VAN VUURDE, 1990; PEROMBELON et al., 1998), and the detection of known DNA sequences following amplification by the PCR using pathogen specific oligonucleotide primers (NIEPOLD, 1994; DE BOER and WARD, 1995; SMID et al., 1995; HYMAN et al., 1997; HELIAS et al., 1998).

In this study two sets of primers and different extraction procedures were compared to improve the practice of seed certification by a simple, fast, sensitive and specific method for determining the level of E. carotovora-contamination of seed tubers in order to overcome the problem of inhibition of the polymerase chain reaction by potato tuber derived compounds.

\subsection{Specificity of the two $E c$ primer pairs}

The specificity and reliability of the Eca primers to amplify a fragment of Erwinia carotovora were verified by testing different bacterial strains. In the first screening step, two primer sets were tested for their ability to differentiate the two Erwinia subspecies. For comparison 5 Eca and 4 Ecc strains were tested, which can infect potatoes. The results showed that the primers Y1 and Y2 amplified DNA from all Eca and Ecc strains tested and confirmed previous reports of HELIAS et al. (1998) who found that PCR performed on 140 Erwinia strains yielded an amplification product of $434 \mathrm{bp}$ in 109 strains from Eca and Ecc only, whereas none of the surveyed E. carotovora subsp. betavasculorum strains showed an amplificate. Also, BDYLIA (1995) obtained similar results when different strains of Eca, Ecc and Ech were tested by these primers. In his studies, only $E c a$ and $E c c$ gave a product of 434 bp, while Ech strains did not show amplificates. 
In contrast, the primers ECAIf/ECA2r were suited to specifically detect the blackleg pathogen $E c a$. We confirmed by our experiments that the $690 \mathrm{bp}$ fragment from Eca strains was not amplified when using DNA from Ecc strains. The same results were obtained by DE BOER and WARD (1995) who amplified a $690 \mathrm{bp}$ fragment from Eca strains but not from strains of other Erwinia carotovora subspecies isolated from various hosts.

\subsection{Determination of the detection limit in pure bacterial cultures of Erwinia carotovora}

Our results obtained in this study revealed that it is not necessary to extract and purify bacterial DNA from pure cultures. The specific DNA amplificates were detected from all Eca and Ecc strains tested. The PCR performed on boiled bacterial suspensions yielded a $434 \mathrm{bp}$ fragment by the $E c a$ and $E c c$-specific primers Y1 and Y2, whereas a $690 \mathrm{bp}$ fragment was obtained by the ECA1f/ ECA2r primers, specific for Eca.

The detection limit determined in these studies was similar for the two primers $\left(\mathbf{1 0}^{\mathbf{3}} \mathbf{c f u} / \mathbf{m l}\right)$, as confirmed by plating aliquots of serial dilutions onto King's medium B. Boiling of bacterial suspensions proved to be a useful step prior to PCR assays because of its easiness. Previous publications (BDLIYA, 1995; HYMAN et al., 1997) reported detection limits of bacterial suspensions after extracting the DNA, which were similar to our results with Erwinia spp.. Thus, BDLIYA (1995) determined a detection limit of $10^{4}-10^{5} \mathrm{cfu} / \mathrm{ml}$ which was still within the range reported earlier (ANONYMOUS, 1992; LÜDTKE, 1994). Our results confirmed those obtained by MARTINS (2000) who reported a detection limit of $4 \times 10^{2} \mathrm{cfu} / \mathrm{ml}$ from bacterial suspensions of Ralstonia solanacearum, while NIEPOLD (1994) could detect only $10^{4} \mathrm{cfu} / \mathrm{ml}$ of $E c a$.

It is concluded that boiling of bacteria pure cultures is a simple and cheap method requiring very little equipment. Only $5 \mathrm{~min}$ are needed to boil the bacterial suspensions in contrast to 4 $\mathrm{h}$ or more required for most of the DNA extraction procedures.

\subsection{Comparison of four DNA extraction methods}

This study describes a PCR assay for specifically detecting Eca in potato tubers. As peel extract contains PCR inhibitory substances (VAN DER WOLF et al., 1996), it was necessary to extract DNA beforehand, taking the opportunity to concentrate it and, thus, to increase sensitivity of the assay. In the past few years, PCR assays based on three different sets of DNA primers have been developed for detecting contamination by Erwinia carotovora. 
The method of LI and DE BOER (1995) involving maceration in Tris-EDTA buffer, washing with cold acetone, purification with proteinase and ammonium aceteate precipitation of cell debris, and DNA precipitation with isopropanol allowed to obtain high yielding bacterial DNA from potato peel extracts. The procedure used by LI and DE BOER to prepare DNA for PCR amplification, avoids the use of organic solvents for separating DNA from cell debris. This method was relatively easy to use on a large number of samples. Successful amplification of DNA extracted from peel extracts indicated a wide applicability of the PCR test. This DNA purification method proved to be advantageous since the extract did not contain plant derived compounds which inhibited amplification of DNA in peel extract in comparison to other protocols described in 2.3 which were not successful in this study. The centrifugation of the macerated peel extract showed to be a useful step in the DNA purification procedure.

In the here reported PCR experiments, inhibitory substances occurred in undiluted samples and 1:10 diluted homogenates of potato peel extract (Fig. 4 and Fig. 5). The inhibiting effect of potato tissue, probably by phenolic compounds, appeared to be strong. Thus, ELPHINSTONE et al. (1996) reported that the PCR product could not be amplified when macerates of potato cv. Desire' were used. FRECHON et al. (1998) reported that evaluation in five different laboratories using similar DNA extraction procedures confirmed the sensitivity of this method, which ranged from 10 to $10^{3} \mathrm{cfu} / \mathrm{ml}$ of $E c a$ depending on the $E c a$ strain, potato cultivars/stocks and the speed and ease of procedure. The sensitivity of PCR when detecting bacteria directly in crude peel extracts, was ca. $10^{6} \mathrm{cfu} / \mathrm{ml}$ (VAN DER WOLF et al., 1996). This low sensitivity was attributed to the presence of inhibitory compounds in peel extracts.

\subsection{Determination of the detection limit of $E c$ in potato peel extracts}

The detection limit by PCR obtained in this study, when determined by plating aliquots of serial dilutions on the semi-selective medium, was $\mathbf{1 0}^{\mathbf{2}} \mathbf{c f u} / \mathbf{m l}$ peel extract. PCR detection avoids the difficulty of different serogroups within $E c$ by using $E c a$-specific primers (DE BOER and WARD, 1995; FRECHON et al., 1998; SMID et al., 1995) detecting all serogroups of $E c$. However, the sensitivity in plant material has been limited, since concentrations of $1 \mathrm{X}$ $10^{5}-1 \times 10^{8}$ cells $/ \mathrm{ml}$ peel extract were needed to obtain positive PCR signals (FRAIIJE et al., 1996; VAN DER WOLF et al., 1996; HELIAS et al., 1998). These detection thresholds can be greatly improved by either enrichment or immunomagnetic separation of the bacteria before PCR detection, which allows a detection of $10^{4}$ cells $/ \mathrm{ml}$ peel extract (FRAIIJE et al., 1996) down to $2 \times 10^{3}$ cells $/ \mathrm{ml}$ peel extract (VAN DER WOLF et al., 1996). The lower sensitivity of PCR for peel extract was due to the presence of inhibitory compounds in the potato peel extract prepared. Since we applied the DNA extraction method of LI and DE BOER (1995) excluding 
the inhibitory compounds, the sensitivity could be improved to detect $\mathbf{1 0}^{\mathbf{2}} \mathbf{c f u} / \mathbf{m l}$ peel extract. By comparing the detection limit from contaminated potato peel extract to that from bacterial pure cultures it appeared that the detection limit from peel extract was a little bit lower $\left(10^{2}\right.$ $\mathrm{cfu} / \mathrm{ml})$ than that from the pure culture $\left(1.5 \times 10^{3} \mathrm{cfu} / \mathrm{ml}\right)$. This difference may be due to the extraction methods applied. The bacterial pure culture were only boiled for $5 \mathrm{~min}$, whereas the potato peel homogenate was extracted by acetone and EDTA (see IV, 2.5.1). By the latter method the DNA is concentrated which increases the sensitivity of the test.

\subsection{Comparison of microbiological and molecular genetic methods for detection of Erwinia carotovora on or in potatoes}

The PCR assay is the most specific method for detecting of E. carotovora. It also has a satisfactory sensitivity level and can be completed within one day. By comparing the PCR method with the semi-selective medium used in this study it can be concluded that the detection limit obtained was a slightly lower by the microbiological method $\left(2 \times 10^{1} \mathrm{cfu} / \mathrm{ml}\right)$ (Chapter I, see 3.3$)$ than by the PCR $\left(10^{2} \mathrm{cfu} / \mathrm{ml}\right)$.

However, the PCR method requires more specialized facilities than the microbiological method, especially if carry-over of contaminations from previous amplification is to be avoided. These include separate containment facilities such as biosafety cabinets, dedicated laboratory equipment, use of ultra-pure water, pipette tips with filters. Furthermore, costs tend to be high, since not only expensive equipment and reagents are needed but this method requires more labor time than the microbiological method. In addition, the latter method allows to quantify the degree of contamination. The disadvantage of the semi-selective medium is the difficulty to differentiate between Eca and Ecc.

In conclusion, the microbiological method proved to be very sensitive, and thus should be recommended as a standard procedure for detection of Erwinia carotovora strains. In contrast, the PCR technique can be a useful additional method and is very valuable for differentiating $E c a$ from $E c c$ isolates. 


\section{SUMMARY}

1 - The aim of this study was to compare two specific E. carotovora primer sets to determine the method best suited for detecting latent contamination of potato tubers.

2 - The specificity of primers Y1 and Y2 was broader by detecting Eca and Ecc, whereas primers Eca1f and Eca2r detected only Eca.

3 - The results revealed that the detection limit of pure cultures of $E c a$ was $1.5 \times 10^{3} \mathrm{cfu} / \mathrm{ml}$ for both primer pairs Y1 \& Y2 and Eca1f \& Eca2r when the bacterial suspensions were boiled for 5 min without any DNA-extraction.

4 - The detection limit from potato peel extract contaminated by $E c a$ was $\mathbf{1 0}^{\mathbf{2}} \mathrm{cfu} / \mathrm{ml}$ by both primer sets (Y1/ Y2 and Eca1f/Eca2r) when the DNA-extraction method of LI and DE BOER was applied.

5- Considering the costs and requirements for a PCR detection the use of the semi-selective agar medium appears to be more advantageous for detecting and quantifying latent contamination of potato tubers by $E c a$ and $E c c$. 


\section{LIST OF REFERENCES}

ADINOLFI, M., CORSARO, M.M., DE CASTRO, C., LANZETTA, R., PARRILli, M., EVIDENTE, A. and LAVERMICOCCA, P. (1995a): A novel 4-C-branched sugar from lipopolysaccharide of the bacterium Pseudomonas caryophylli. Carbohydr. Res. 267, 307 311.

ADINOLfI, M., CORSARO, M.M., DE CASTRO, C., EVIDENTE, A., LANZETTA, R., MANGONI, L. and PARRILLI, M. (1995b): The relative and absolute configurations of stereocenters in caryophyllose. Carbohydr. Res. 274, 223 - 232.

AHMED, M.E., MAVRIDIS, A. and RUDOLPH, K. (2000): Detection of latent contamination of potato tubers by Erwinia spp. using a semi-selective agar medium. Phytomedizin, Mitteilung. Deutsch. Phytomedizin. Gesellsch. 30 (1), 15 - 16.

ALEXANDER, C. and RIETSCHEL, E.T. (1999): Bakterielle Lipopolysaccharide -hochaktive Stimulatoren der angeborenen Immunität. Bio spektrum 4, 275 - 281.

ALLAN, E. and KELMAN, A. (1977): Immunofluorescent stain procedures for detection and identification of Erwinia carotovora subsp. atroseptica. Phytophathology 67, 1305 - 1312.

ALlEFS, S., VAN DOOIJEWEERT, W., DE JONG, E.R, PRUMMEL, W. and HOOGENDOON, C. (1995): The role of the seed tuber in determining partial resistance to potato blackleg caused by Erwinia spp.. Eur. J. Plant Pathology 101,189 - 199.

ALIPPI, A.M., DALBO, E., RONCO, L.B. and CASANOVA, P.E. (1997): Tomato as a new host of Erwinia carotovora subsp. carotovora in Argentina. Plant Disease 81, 230.

AL-SHINAWI, T. (1996): Untersuchungen zur Epidemiolgie von Pseudomonas syringae pv. coriandricola, dem Erreger des bakteriellen Doldenbrandes an Koriander (Coriandrum sativum L.), und zu seiner Bekämpfung durch resistente Linien. Dissertation, Universität Göttingen, $141 \mathrm{pp}$. 
ANONYMOUS (1992): COST 88, workshop on immunological and PCR-based methods to detect and quantify populations of Erwinia carotovora subsp. atroseptica on potato tubers. 22 - 26 Sept. 1992, Scottish Crop Research Institute, Invergowrie, Dundee Scotland, U. K. p. 35.

ATLAS, R.M., SAYLER, G., BURLAGE, R.S. and BEI, A.K. (1992): Molecular approaches for environmental monitoring of microorganisms. Biotechniques 12, 706.

BARRAS, F., VAN GIJSEGEM, F. and CHATTERJEE, A.K. (1994): Extracellular enzymes and pathogenesis of soft rot Erwinia. Ann. Rev. Phytopathol. 32, $201-234$.

BATEMAN, D.F. and MILlAR, R.L. (1966): Pectic enzymes in tissues degradation. Ann. Rev. Phytopathol. 4, $119-139$.

BEI, A.K., MAHBUBANI, M.H., DICESARE, J.L. and ATLAS, R.M. (1991): Polymerase chain reaction-gene probe detection of microorganisms by using filter-concentrated samples. Appl. Enviro. Microbiol. 57, 3529 - 3534.

BDLIYA, B.S. (1995): Studies on the detection and identification of soft rot causing Erwinia species (Erwinia carotovora ssp. atroseptica (van Hall) Dye, Erwinia carotovora ssp. carotovora (Jones) Bergey et al., and Erwinia chrysanthemi Burkholder et al.) on potato tubers. Dissertation, Universität Göttingen, 148 pp.

BERAHA, L. (1968): A rapid method for the preparation of semi-solid agar medium for detection of pectolytic enzyme activity in Erwinia carotovora. Plant. Dis. Rep. 52, 167.

BOURGEOIS, F.R. and PRIOU, S. (1995): Screening tuber-bearing Solanum spp. for resistance to soft rot caused by Erwinia carotovora ssp. atroseptica (van Hall) Dye. Potato Research 38, $111-118$.

BRADBURY, J.F. (1986): Guide to Plant Pathogenic Bacteria. C. A. B. International, Mycological Institute, Ferry Lane, Kew, Surrey, England, p. 66 - 71.

BRAUN, V. and SCHMITZ, G. (1980): Excretion of a protease by Serratia marcescens. Arch. Microbiol. 124, $55-61$. 
CHATTERJEE, A.K., BUCHANAN, G.E., BEHRENS, M.K. and STARR, M.P. (1979): Synthesis and excretion of polygalacturonic acid and trans-eliminase in Erwinia, Yersinia, and Klebsiella species. Can. J. Microbiol. 25, 94 - 102.

CHATTERJEE, A.K. and STARR, M.P. (1980): Genetics of Erwinia species. Ann. Rev. Microbiol. 34, $645-676$.

CHIU, W.-F., DI, Y.-P., CHOW, Y.-Y. and SI, F.-J. (1965): Some bacteria causing decay of Chinese cabbage in storage. Rev. Appl. Mycol. 44, 2273.

CIAMPI-PANNO, L. and ANDRADE-SOTO, N. (1984): Preliminary evaluation of bacterial soft rot resistance in native Chilean potato clones. American Potato J. 61, $109-112$.

CIUCANU, I. and KEREK, F. (1984): A simple and rapid method for the permethylation of carbohydrates. Carbohydrate Res. 131, 209 - 217.

CLARK, M., HOPY,W., BOND, J.P., CHEN, C., GOH, X., LIANG, X. and LOTRAKUL, P. (1998): First report of Erwinia carotovora subsp. carotovora causing bacterial root rot of sweetpotato in Louisiana. Plant Disease 82, 129.

COLLMER, A. and BAUER, D.W. (1994): Erwinia chrysanthemi and Pseudomonas syringae: plant pathogens trafficking in extracellular virulence proteins. In: Bacterial Pathogenesis of Plants and Animals, ed. JL Dangl, pp. 43 - 78.

COLLMER, A. and KEEN, N.T. (1986): The role of pectic enzymes in plant pathogenesis. Ann. Rev. Phytopathol. 24, 383 - 409.

COSTERTON, J.W., INGRAM, J. M. and CHENG, K.- J. (1974): Structure and function of the cell envelope of Gram-negative bacteria. Bacteriol. Rev. 38, 87 - 110.

COTHER, E.I., BLAKENEY, A.B. and LAMB, S.J. (1980): Laboratory-scale preparation of sodium polypectate for use in selective media for pectolytic Erwinia ssp. Plant Disease 64, $1086-1087$. 
COTHER, E.J. (1980): Bacterial seed tuber decay in irrigated sandy soils of New South Wales. Potato Research 23, $75-79$.

CROMARTY, R.W. and EASTON, G.D. (1973): The incidence of decay and factors affecting bacterial soft rot of potatoes. American Potato J. 50, 398 - 407.

CUPPELS, D. and KELMAN, A. (1974): Evaluation of selective media for isolation of soft rot bacteria from soil and plant tissue. Phytophathology 64, $468-475$.

DARLING, D.C. and BRICKELL, P.M. (1994): Nucleic Acid Blotting - The Basis. IRL Press, $111 \mathrm{pp}$.

DAZZO, F.B., TRUCHET, G.L., HOLLINGSWORTH, R.I., HRABAK, E.M., PANKRATZ, H.S., PHILIP- HOLLINGSWORTH, S., SALZWEDEL, J.L., CHAPMAN, K., APPENZELlER, L., SQUARTINI, A., GERHOLD, D. and ORGAMBIDE, G. (1991): Rhizobium lipopolysaccharide modulates infection thread development in white clover root hairs. J. Bacteriol. 173, 5371 - 5384.

DE BOER, S.H., BRADSHAW-ROUSE, J.J., SEQUEIRA, L. and Mc NAUGHTON, M.E. (1985): Sugar composition and serological specificity of Erwinia carotovora lipopolysaccharides. Can. J. Microbiol. 31, $583-586$.

DE BOER, S.H., COPEMAN, R.J. and VRUGGINK, H. (1979): Serogroups of Erwinia carotovora potato strains determined with diffusible somatic antigens. Phytopathology 68, $1784-1790$.

DE BOER, S.H. and KELMAN, A. (1975): Evaluation of procedures for detection of pectolytic Erwinia spp. on potato tubers. American Potato J. 52, 117 - 123.

DE BOER, S.H. and Mc NAUGHTON, M.E. (1987): Monoclonal antibodies of the lipopolysaccharide of Erwinia carotovora subsp. atroseptica serogroup I. Phytopathology 77, $828-832$.

DE BOER, S.H., SLACK, S.A., VAN DEN BOVENKAMP, G.W. and MASTENBROEK, I. (1996): A role for pathogen indexing procedures in potato certification. In: ANDREWS, J.H., 
S.H. DE BOER, I.C. TOMMERUP (eds), Pathogen indexing technologies, Advances in Botanical Research vol. 3 pp. 217 - 242. Academic Press, London.

DE BOER, S.H. and WARD, L.J. (1995): PCR detection of Erwinia carotovora subsp. atroseptica associated with potato tissue. Phytopathology 85, $854-858$.

DÖPKE, F. and RUDOLPH, K. (1990): Screening for resistance to soft rot. In: Methods in Phytobacteriology. KLEMENT, Z., RUDOLPH, K. and SANDS, D. C. eds. Akademiai Kaido Budapest, 1990, pp. $349-353$.

EDWARDS, P.R. and EWING, W.H. (1972): Identification of Enterobacteriaceae. 3rd ed. Burgess Publishing Co., Minneapolis.

El-KAZAZZ, S.A.I. (1984): Physiological studies on soft-rot bacteria with special reference to the possible production of toxins. Disseration, Alexandria University, 138 pp.

ELPHINSTONE, J.G., HENNESSY, J., WILSON, J.K. and STEAD, D.E. (1996): Sensitivity of different methods for the detection of Ralstonia solanacearum in potato tuber extracts. Bulletin OEPP/EPPO Bulletin 26, $663-678$.

EL-SHOUNY, W. (1993): Biosynthesis of polysaccharides by phytopathogenic bacteria. Disseration, Tanta University, $181 \mathrm{pp}$.

FAHY, P.C. and HAYWARD, A.C. (1983): Plant Bacterial Disease (a Diagnostic Guide). Academic Press, Sydney, New York, London, page 349.

FAVEY, S., BOURSON, C., BERTHEAU, Y., KOTOUJANSKY, A. and BOCCARA, M. (1992): Purification of the acidic pectate lyase and nucleotide sequence of the corresponding gene (pel A) of Erwinia chrysanthemi strain 3937. J. G. Microbiol. 138, 499 - 508.

FICKE, W., NAUMANN, K., SKADOW, K., MULLER, H.J. and ZIELKE, R. (1973): Die Lebensdauer von Pectobacterium carotovorum var. atrosepticum (van HALL) DOWSON auf dem Pflanzgut und im Boden. Arch Phytopathol. u Pflanzenschutz 9, 281 - 293. 
FRAAIJE, B.A., APPELS, M., DE BOER, S.H., VAN VUURDE, J.W.L. and VAN DEN BULK, R.W. (1997): Detection of soft rot Erwinia spp. on seed potatoes: conductimetry in comparison with dilution plating, PCR and serological assays. Eur. J. Plant Pathol. 103, 183 193.

FRAAIJE, B., BIRNBAUM, Y. and VAN DEN BULK, R.W. (1996): Comparison of methods for detection of Erwinia carotovora ssp. atroseptica in progeny tubers derived from inoculated tubers of Solanum tuberosum L. J. Phytopathol. 144, 551 - 557.

FRECHON, D., EXBRAYAT, P., GALLET, O., GUILLOT, E.L.E., ClERC, V., PAYET, N. and BERTHEAU, Y. (1998): Sequences nucleotidiques pour la detection des Erwinia carotovora subsp. atroseptica. Brevet 95. 12 - 803 INRA INA P-G, SDP. Paris (FR).

FUKUOKA, S., KAMISHIMA, H., SODE, K. and KARUBE, I. (1989): Extracellular lipopolysaccharide production by Erwinia carotovora. J. Fermentation Bioenginering 68, 320 $-324$.

FUKUOKA, S., KAMISHIMA, H., SODE, K. and KARUBE, I. (1990): Facile isolation of endo-pectate lyase from Erwinia carotovora basesd on electrostatic interaction. Appl. Biochem. Biotechnol. 26, 239 - 248.

FUKUOKA, S., KAMISHIMA, H., NAGAWA, Y., NAKANISHI, H., ISHIKAWA, K., NIWA, Y., TAMIYA, E. and KARUBE, I. (1992): Structural characterization of lipid A component of Erwinia carotovora lipopolysaccharide. Arch. Microbiol. 157, 311 - 318.

FUKUOKA, S., KNIREL, Y.A., LINDNER, B., MOLL, H., SEYDEL, U. and ZÄHRINGER, U. (1997): Elucidation of the structure of the core region and the complete structure of the Rtype lipopolysaccharide of Erwinia carotovora FERM P-7576. Eur. J. Biochem 250, 55 - 62.

FUKUOKA, S., KODAMA, M. and KARUBE, I. (1995): Thermotropic phage behavior of membranes made from Erwinia carotovora rough form lipopolysaccharide, Thermochim. Acta 257, $93-102$.

GARDNER, J.M. and KADO, C.I. (1976): Polygalacturonic acid trans-eliminase in the 
osmotic shock fluid of Erwinia rubrifaciens: characterization of the purified enzyme and its effect on plant cells. J. Bacteriol. 127, $451-460$.

GERWE, P.-G., RUDOLPH, K. and KÖHN, S. (1987): Vergleich eines "glatten” und eines "rauhen" stammes von Pseudomonas syringae pv. phaseolicola. J. Phytopathol. 118, 326 334.

GLENN, A.R. (1976): Production of extracellular proteins by bacteria. Ann. Rev. Microbiol. 30, $41-62$.

GOTTLIEB, S.F. (1971): Effect of hyperbaric oxygen on microorganisms. Ann. Rev. Microbiol. 25, $111-152$.

GRAHAM, D.C. and HARDIE, J.L. (1971): Prospects for control of potato blackleg disease by the use of stem cuttings. Proc. 6th Br. Insectic . Fungic. Conf . pp. $219-224$.

GREGORY, E. and FRIDOVICH, I. (1973): Oxygen toxicity and superoxide dismutase. J. Bacteriol. 114, 1193 - 1197.

GROLMS, U. (1996): Rheologie der Interaktionen zwischen bakteriellen Lipopolysacchariden (LPS) und pflanzlichen Polymeren und Resistenzinduktion durch LPS. Dissertation, Universität Göttingen, 138 pp.

GROLMS, U. and RUDOLPH, K. (1997): Rheological interactions between lipopolysaccharides of Pseudomonas syringae pathovars and plant polysaccharides. In K. RUDOLPH, T.J. BURR, J.W. MANSFELD, D. STEAD, A. VIVIAN and J. von KIETZELL (edts): Developments in Plant Pathology Vol. 9: Pseudomonas Syringae Pathovars and Related Pathogens. Kluwer Academic Publishers Dordrecht/Boston/London, pp. 364 - 369.

HANNECART-POKORNI, E., DEKEGEL, D. and DEPUYDT, F. (1973): Macromolecular structure of lipopolysaccharides from Gram-negative bacteria. Eur. J. Biochem. 38, 6 - 13.

HARJU, H. and KANKILA, J. (1993): Erwinia carotovora contamination of Finnish seed potatoes and the prevalence of bacterial subspecies and serogroups. Agric. Sci. Finland 2, 345 
$-352$.

HARTSKEER, R.A., DE VIT, M.Y.L. and KLATTSER, P.R. (1989): Polymerase chain reaction for the detection of Mycobacterium leprae. J. Gen. Micobiol. 135, 2357 - 2364.

HELIAS, V., ANDRIVON, D. and JOUAN, B. (2000): Internal colonization pathways of potato plants by Erwinia carotovora ssp. atroseptica. Plant Pathology 49, 33 - 42.

HELIAS, V., LE ROUX, A.C., BERTHEAU, Y., ANDRIVON, D., GAUTHIER, J.P. and JOUAN, B. (1998): Characterization of Erwinia carotovora subspecies and detection of Erwinia carotovora ssp. atroseptica in potato plants, soil and water extracts with PCR-based methods. Eur. J. Plant Pathol. 104, 685 - 699.

HENSEN, J.M. and FRENCH, R. (1993): The polymerase chain reaction and plant disease diagnosis. Ann. Rev. Phytopathol. 31, 81 - 109.

HEUKESHOVEN, J. and DERNIK, R. (1988): Improved silver staining procedure for fast staining in phastsystem development unit. I. Staining of SDS-Gels. Electrophoresis 9, 28 - 32.

HITCHCOCK, P.J., LEIVE, L., MÄKELÄ, H., RIETSCHEL, E.T., STRITTMATTER, W. and MORRISON, D.C. (1986): Minireview: lipopolysaccharide nomenclature past, present and future. J. Bacteriol. 166, $699-705$.

HUGH, R. and RYSCHENKOW, E. (1961): Pseudomonas maltophilia, an alcaligenes-like species. J. Gen. Microbiol. 26, 123 - 132.

HUSSAIN, A. and KELMAN, A. (1958): Relation of slime production to mechanism of wilting and pathogenicity of Pseudomonas solanacearum. Phytopathology 48, 155 - 165.

HYMAN, L.J., DEWASMES, V., TOTH, I.K. and PEROMBELON, M.C.M. (1997): Improved PCR detection sensitivity of Erwinia carotovora subsp. atroseptica in potato tuber peel extract by prior enrichment on a selective medium. Letters Appl. Microbiol. 25, 143 147. 
JANSE, J.D. and SPIT, B.E. (1989): A note on the limitations of identifying soft rot erwinias by temperature tolerances and sensitivity to erythromycin on a pectate medium. J. Phytopathology 125, $265-268$

JANSSON, P.-E., KENNE, L., LINDBERG, B., LIEDGREN, H. and LÖNNGREN, J. (1976): A practical guide to methylation analysis of carbohydrates. Chem. Commun. Univ. Stockholm, $8,1-75$.

JONES, D.A.C., HYMAN, L.J., TUMESEIT, M., SMITH, P. and PEROMBELON, M.C.M. (1994): Blackleg potential of potato seed: determination of tuber contamination by Erwinia carotovora subsp. atroseptica by immunofluorescence colony staining and stock and tuber sampling. Ann. Appl. Biol. 124, 557 - 568.

JONES, D.A.C., McLEOD, A., HYMAN, L.J. and PEROMBELON, M.C.M. (1993): Specifity of an antiserum against Erwinia carotovora subsp. atroseptica in indirect ELISA. J. Appl. Bacteriol. 74, $620-624$.

KAMIUNTEN, H. and FUJITA, T. (1990): Electrophoretic analysis of the lipopolysaccharide from Xanthomonas campestris pv. oryzae and Pseudomonas avenae. Kyushu Byogaichu Kenkyukaiho 36, $14-15$.

KANKILA, J. (1989): Methods for the detection of serologically heterogeneous populations of Erwinia carotovora subsp. atroseptica. In: Plant Pathogenic Bacteria. Part B, pp. 889 - 894. Ed. Z. KLEMENT. Budapest, Hungary: Akademiai Kiado.

KELLER, H. and KNÖSEL, D. (1980): Untersuchungen über die bakterielle Strunkfäule des Lagerkohls. Nachrichtenbl. Deut. Pflanzenschutzd. 32, $161-163$.

KELMAN, A., BAUGHM, J.W. and MAHER, E.A. (1978): The relationship of bacterial soft rot susceptibility to water status of potato tubers (Abstract). Phytopathology News 12, 178.

KELMAN, A. and DICKEY, R.S. (1980): Soft rot or „carotovora“ group. - In: Laboratory Guide for the Identification of Plant Pathogenic Bacteria (ed. SCHAAD, N.W.). American Phytopathological Soc., St. Paul: pp. $31-35$. 
KING, E.O., WARD, M.K. and RANEY, D.E. (1954): Two simple media for the demonstration of pyocyanin and fluorescin. J. Lab. Clin. Med. 44, 301 - 307.

KNIREL, Y.A. and KOCHETKOV, N.K. (1994): The structure of lipopolysaccharides of Gram-negative bacteria. III. The structure of O-antigens: a review. Biokhimya 59, 1325 1382.

KNIREL, Y.A., VEREMEYCHENKO, S.N., ZDOROVENKO, G.M., SHASHKOV, A.S., PARAMONOV, N.A., ZAKHAROVA, I.Y. and KOCHETKOV, N.K. (1994): Somatic antigens of pseudomonads: structure of the O-specific polysaccharide of Pseudomonas fluorescens biovar A strain IMV 472. Carbohydr. Res. 259, 147 - 151.

KNIREL, Y.A. and ZDOROVENKO, G.M. (1997): Structures of O-polysaccharide chains of lipopolysaccharides as the basis for classification of Pseudomonas syringae and related strains. In: K. RUDOLPH, T. BURR, J.W. MANSFIELD, D. STEAD, A. VIVIAN and J. Von KIETZELL (eds.), Pseudomonas Syringae Pathovars and Related Pathogens. pp. 475 - 480. Kluwer Academic Press. Dordrecht, Netherlands.

KOTOUJANSKY, A. (1987): Molecular genetics of pathogenesis by soft-rot erwinias. Ann. Rev. Phytopathol. 25, $405-430$.

LANGERFELD, E. (1984): Naßfäule und Schwarzbeingkeit der Kartoffel aus der Sicht jüngerer Forschungsergebnisse. - Eine Literaturübersicht. Nachrichtenbl. Deut. Pflanzenschutzd. 36, $97-103$.

LAPWOOD, D.H. and GANS, P.T. (1984): A method for assessing the field susceptibility of potato cultivars to blackleg (Erwinia carotovora subsp. atroseptica). Annals Appl. Biol. 104, $315-320$.

LAPWOOD, D.H., READ, P.J. and SPOKES, J. (1984): Methods for assessing the susceptibility of potato tubers of different cultivars to rotting by Erwinia carotovora subsp. atroseptica and carotovora. Plant Pathology 33, $13-20$. 
LAUX, P. (1998): Zur Rolle der Lipopolysaccharide (LPS) in der Wirt/Parasit-Interaktion phytopathogener Pseudomonaden und Xanthomonaden. Dissertation, Universität Göttingen, $165 \mathrm{pp}$.

LAUX, P., MÜLlER, P. and RUDOLPH, K. (1998): Interactions between bacterial lipopolysaccharides and plant pectins, a mechanisms which may determine host/parasite relations in bacterial plant diseases. In Mahadevan, (ed.): Plant Pathogenic Bacteria, Proceed. $9^{\text {th }}$ Internation. Conf., Centre for Advanced Study in Botany, Univ. Madras, Chennai-India, pp. $563-568$.

LAZAR, I. and BUCUR, E.L. (1964): Recent research in Romania on blackleg and bacterial soft rot of potato. European Potato. J. 7, $102-111$.

LELLIOTT, R.A. (1974): Genus XII Erwinia. In Bergey's Manual of Determinative Bacteriology (Eds. R. E. Buchanan and N. E. Gibbons), pp. 332 - 339.

LELLIOTT, R.A. and STEAD, D.E. (1987): Methods for the Diagnosis of Bacterial Diseases of Plants, Vol. 2. Blackwell Scientific Publication, Oxford, London, p. 216.

LEONTEIN, K., LINDBERG, B. and LÖNNGREN, J. (1978): Assignment of absolute configuration of sugars by g.l.c. of their acetylated glycosides formed from chiral alcohols. Carbohydr. Res. 62, $359-362$.

LI, X. and DE BOER, S.H. (1995): Selection of polymerase chain reaction primers from an RNA intergenic spacer region for specific detection of Clavibacter michiganesis ssp. sepedonicus. Phytopathology 85, $837-842$.

LINDSAY, S.S., WHEELER, B., SANDERSON, K.E., COSTERTON, J.W. and CHENG, K.J. (1973): The release of alkaline phosphatase and of lipopolysaccharide during the growth of rough and smooth strains of Salmonella typhimurium. Can. J. Microbiol. 19, 335 - 343.

LOGAN, C. (1963): A selective medium for isolation of soft rot coliforms from soils. Nature (London) 199, 623. 
LÜDERITZ, O., FREUDENBERG, M.A., GALANOS, O., LEHMANN, V., RIETSCHEL, E.T. and SHAW, D.H. (1982): Lipopolysaccharides of Gram-negative bacteria. Curr. Top. Membr. Transp. 17, $79-151$.

LÜDTKE, J.-D. (1994): Untersuchungen über ein molekulargenetisches Testverfahren zum sicheren Nachweis von Clavibacter michiganesis ssp. sepedonicus (Cms) dem Erreger der bakteriellen Ringfäule der kartoffel. Diplomarbeit. Fachbereich Agrarwissenschaften der Universität Göttingen, Germany.

LUND, B.M. (1979): Bacterial soft-rot of potatoes. In Soc. Appl. Bacteriol. Tech. Ser. 12. Plant Pathogens, ed. D. W. LOVELOCK, R. DAVIES, pp. 14 - 49.

MARTINS, O.M. (2000): Polymerase chain reaction in the diagnosis of bacterial wilt, caused by Ralstonia solanacearum (Smith) Yabuuchi et al. Dissertation, Universität Göttingen, 127 pp.

MIELKE, H. and HOPPE, H. (1982): Investigations into the influence of peracetic acid on foot rot disease of cereals. J. Plant Dis. and Prot. 89, 282 - 290.

MÖLLER, E.M., BAHNWEG, G., SANDERMANN, H. and GEIGER, H.H. (1992): A simple and efficient protocol for the isolation of high molecular weight DANN from filamentous fungi, fruit bodies, and infected plant tissues. Nucleic Acid Res. 20, 6115-6116.

MORAN, F. and STARR, M.P. (1969): Metabolic regulation of polygalacturonic acid transeliminase in Erwinia. European J. Biochem. 11, 291 - 295.

MÜLLER, P. (1998): Zur Wirkungsweise und Struktur bakterieller Lipopolysaccharide (LPS) aus Pseudomonas syringae Pathovarietäten bei der induzierten Resistenz in Nicotiana tabacum und Lycopersicon esculentum. Disseration, Universität Göttingen, 135 pp.

MÜLLER, P., ZÄHRINGER, U. and RUDOLPH, K. (1998): Induced resistance by bacterial lipopolysaccharides (LPS). In Mahadevan, (ed.): Plant Pathogenic Bacteria, Proceed. $9^{\text {th }}$ Internation. Conf., Centre for Advanced Study in Botany, Univ. Madras, Chennai-India, pp. $569-575$. 
MUNZERT, M. and HUNNIUS, W. (1980): Relation between the resistance to Erwinia carotovora and Fusarium spp. in the potato. Z. Pflanzenzüchtung 85, 59-70.

MURATA, H., FONS, M., CHATTERJEe, A., COLlMER, A. and CHATTERJEE, A.K. (1990): Characterisation of transposon insertion out mutants of Erwinia carotovora subsp. carotovora defective in enzyme export and of a DNA segment that complements out mutants in Erwinia carotovora subsp. carotovora, Erwinia carotovora subsp. atroseptica and E. chrysanthemi. J. Bacteriol. 172, 2970 - 2978.

MURRAY, J., FIXTER, L.M., HAMILTON, I.D., PEROMBELON, M.C.M., QUINN, C.E. and GRAHAM, D.C. (1990): Serogroups of potato pathogenic Erwinia carotovora strains: identification by lipopolysaccharide electrophoresis patterns. J. Appl. Bacteriol. 68, 231 - 240.

NAUMANN, K., ZIELKE, R., PETT, B., STACHEWICZ, H. and JANKE, C. (1976): Umfang und Bedeutung des latenten Befalls von Kartoffelknollen mit Pectobacterium carotovora var. atroseptica. Tag. Ber. ADL 140, $231-241$.

NEWMAN, M.-A., DANIELS, M.J. and DOW, J.M. (1995): Lipopolysaccharide from Xanthomonas campestris induced defense-related gene expression in Brassica campestris. Mol. Plant Microbe Interact. 8, 778 - 780.

NIELSON, L.W. (1978): Erwinia species in the lenticels of seed potatoes. American Potato J. $55,671-676$.

NIEPOLD, F. (1994): Anwendung der Polymerase-Kettenreaktion (PCR) zum Nachweis von Erwinia carotovora ssp. atroseptica, dem Erreger der Schwarzbeingkeit und Nassfäule bei Kartoffeln. Nachrichtenbl. Deut. Pflanzenschutzd. 46, 243 - 246.

NIEPOLD, F. (1999a): A simple and fast extraction procedure to obtain amplifyable DNA from Ralstonia (Pseudomonas) solanacearum and Clavibacter michiganesis ssp. sepedonicus inoculated potato tuber extracts and naturally infected tubers to conduct a polymerase chain reaction (PCR). J. Phytopathol. 147, 249 - 256. 
NIEPOLD, F. (1999b): Efficiency surveys of the peracids Degaclean and Clarmarin in combination with catalase inhibitor KH10 from Degussa company for eradicating the two quarantine bacteria Clavibacter michiganesis ssp. sepedonicus and Ralstonia solanacearum in an aqueous suspension and in the sewage water of the starch industry. J. Phytopathol. 147, 625 -634 .

NIKAIDO, H. and VAARA, M. (1985): Molecular basis of bacterial outer membrane permeability. Microbiol. Rev. 49, 1 - 32 .

OHNO, N. and MORRISON, D.C. (1989): Lipopolysaccharide interaction with lysozyme. J. Biol. Chem. 264, $4434-4441$.

OKON, J. (1990): Methods in agronomy to reduce bacterial diseases. In: Methods in Phytobacteriology. KLEMENT, Z., RUDOLPH, K. and SANDS, D. C. eds. Akademiai Kaido Budapest, 1990, pp. $301-306$.

OVOD, V., KNIREL, Y.A., SAMSON, R. and KROHN, K. (1999): Immunochemical characterization and taxonomic evaluation of the $\mathrm{O}$ polysaccharides of the lipopolysaccharides of Pseudomonas syringae Serogroup 01 strains. J. Bacteriol. 181, 6937 - 6947.

OVOD, V., RUDOLPH, K., KNIREL, Y.A. and KROHN, K. (1996): Immunochemical characterization of O-polysaccharides composing the $\propto-\mathrm{D}$-rhamnose backbone of lipopolysaccharide of Pseudomonas syringae and classification of bacteria into serogroups 01 and 02 with monoclonal antibodies. J. Bacteriol. 178, $6459-6465$.

OVOD, V., RUDOLPH, K. and KROHN, K. (1997): Serological classification of Pseudomonas pathovars based on monoclonal antibodies towards the lipopolysaccharide Ochain. In: K. RUDOLPH, T. J. BURR, J. W. MANSFIELD, D. STEAD, A. VIVIAN and J. von KIETZELL (eds.): Developments in Plant Pathology Vol. 9: Pseudomonas Syringae Pathovars and Related Pathogens. Kluwer Academic Publishers, Dordrecht/Boston/London, pp. 526 531. 
PAGEL, W. and HEITEFUSS, R. (1989): Calcium content and cell wall polygalacturonase in potato tubers of cultivars with different susceptibilities to Erwinia carotovora subsp. atroseptica. Physiol. Mol. Plant Pathol. 35, $11-21$.

PAGEL, W. and HEITEFUSS, R. (1990): Enzyme activities in soft rot pathogenesis of potato tubers: Effects of calcium, $\mathrm{pH}$, and degree of pectin esterification on the activities of polygalacturonase and pectate lyase. Physiol. Mol. Plant Pathol. 37, 9 - 25.

PALLERONI, N.J. and BRADBURY, J.F. (1993): Stenotrophomonas, a new bacterial genus for Xanthomonas maltophilia (Hugh 1980) Swings et al., 1983. International J. System. Bacteriol. 43, $606-609$.

PASTRIK, K.-H. and RAINEY, F.A. (1999): Identification and differentiation of Clavibacter michiganensis subspecies by polymerase chain reaction. J. Phytopathol. 147, 687 - 693.

PEROMBELON, M.C.M. (1971): A quantal method for determining numbers of Erwinia carotovora var. carotovora and Erwinia carotovora var. atroseptica in soils and plant material. J. Appl. Bacteriol. 34, 79 - 3798.

PEROMBELON, M.C.M. (1972): The extent and survival of contamination of potato stocks in Scotland by Erwinia carotovora var. carotovora and Erwinia carotovora var. atroseptica. Ann. Appl. Biol. 71, $111-117$.

PEROMBELON, M.C.M. (1973): Sites of contamination and numbers of Erwinia carotovora present in stored seed potato stocks in Scotland. Ann. App. Biol. 74, $59-65$.

PEROMBELON, M.C.M. (1976): Effects of environmental factors during the growing season on the level of potato tuber contamination by Erwinia carotovora. Phytopathol. Z. 85, 97 116.

PEROMBELON, M.C.M. (1992): Potato blackleg: epidemiology, host-pathogen interaction and control. Neth. J. Pl. Path. 98, Supplement 2, 135 - 146. 
PEROMBELON, M.C.M., BERTHEAU, Y., CAMBER, M., FRECHON, D., LOPEZ, M.M., NIEPOLD, F., PERSSON, P., SLETTEN, A., TOTH, I.K., VAN VUURDE, J.W.L. and VAN DER WOLF, J.M. (1998): Microbiological, immunological and molecular methods suitable for commercial detection and quantification of the blackleg pathogen, Erwinia carotovora subsp. atroseptica, on seed potato tubers: a review. Bulletin OEPP/EPPO Bulletin 28, 141 - 155.

PEROMBELON, M.C.M. and BURNETT, E.M. (1991): Two modified crystal violet pectate (CVP) media for the detection, isolation and enumeration of soft rot erwinias. Potato Research 34, $79-85$.

PEROMBELON, M.C.M., GULlingS-HANDLEY, J. and KELMAN, A. (1979): Population dynamics of Erwinia carotovora and pectolytic Clostridia in relation to decay of potatoes. Phytopathology 69, $167-173$.

PEROMBELON, M.C.M. and HYMAN, L.J. (1986): A rapid method for identifying and quantifying soft rot erwinias directly from plant material based on their temperature tolerances and sensitivity to erythromycin. J Appl. Bacteriol. 60, $61-66$.

PEROMBELON, M.C.M. and HYMAN, L.J. (1989): Survival of soft rot coliforms, Erwinia carotovora subsp. carotovora and E. carotovora subsp. atroseptica in soil in Scotland. J. Appl. Bacteriol. 66, $95-106$.

PEROMBELON, M.C.M. and HYMAN, L.J. (1992): Control of potato blackleg: production of healthy seed. Aspects Appl. Biol. 33, 77 - 84.

PEROMBELON, M.C.M. and HYMAN, L.J. (1995): Serological methods to quantify potato seed contamination by Erwinia carotovora subsp. atroseptica. EPPO Bulletin 25, 195 - 202.

PEROMBELON, M.C.M. and KELMAN, A. (1980): Ecology of the soft rot erwinias. Ann. Rev. Phytopathol. 18, $361-387$.

PEROMBELON, M.C.M. and KELMAN, A. (1987): Blackleg and other potato diseases caused by soft rot erwinias: a proposal for a revision of terminology. Plant Disease 71, 283 285. 
PEROMBELON, M.C.M., LUMB,V. M. and HYMAN, L.J. (1987): A rapid method to identify and quantify soft rot erwinias on seed potato tubers. Eppo Bulletin 18, 25 - 35.

PEROMBELON, M.C.M. and SALMOND, G:P.C. (1995): Bacterial Soft Rots. In: SINGH, U.S., SINGH, R.P. and KOHMOTO, K. (eds),. Pathogenesis and Host Specificity in Plant Diseases. Oxford, UK: Pergamon Press, pp. 1 - 20.

PIERCE, L. and McCAIN, A.H. (1992): Selective medium for isolation of pectolytic Erwinia sp. Plant Disease 76, $382-384$.

PIRHONEN, M., SAARILAHTI, H., KARLSSON, M.B. and PALVA, E.T. (1991): Identification of pathogenicity determinants of Erwinia carotovora subsp. carotovora by transposon mutagenesis. Mol. Plant-Microbe Interact. 4, 276 - 283.

PRIOR, P., ALLEN, C. and ELPHINSTONE, J. (1998): Bacterial Wilt Disease, Molecular and Ecological Aspects, Springer Verlag, Berlin, Heidelberg, New York. 447 pp.

QUANTIC, P., CERVONE, F. and WOOD, R.K.S. (1983): Isoenzymes of a polygalacturonate trans-eliminase produced by Erwinia atroseptica in potato tissue and in liquid culture. Physiol. Plant Pathol. 22, $77-86$.

RAMM, M. (1993): Untersuchungen zur Bildung extrazellulärer polymerer Substanzen mit pathophysiologischer Bedeutung durch Pseudomonas syringae pv. phaseolicola unter besonderer Berücksichtigung extrazellulärer Lipopolysaccharide (LPS). Disseration, Universität Jena.

RAY, T.C., SMITH, A.R.W., WAIT, R. and HIGNETT, R.C. (1987): Structure of the sidechain of lipopolysaccharide from Erwinia amylovora T. Eur. J. Biochem. 170, 357 - 361.

REEVES, P.J., WHITCOMBE, D., WHARAM, S., GIBSON , M., ALLISON, G., BUNCE, N., BARAllon, R., DOUGlas, P., MUlholland, V., STEVENS, S., WALKER, D. and SALMOND, G.P.C. (1993): Molecular cloning and characterisation of 13 out genes from Erwinia carotovora subsp. carotovora: genes encoding members of a general secretion pathway (GSP) widespread in Gram-negative bacteria. Mol. Microbiol. 8, 443 - 456. 
RIETSCHEL, E.T. and BRADE, H. (1992): Bacterial endotoxins. Scientific American, August 1992. pp. $26-33$.

RIETSCHEL, E.T., BRADE, L., SCHADE, F.U., SEYDEI, U., ZÄHRINGER, U., MAMAT, U., SCHMIDT, G., ULMER, A.-J., LOPPNOW, H., FLAD, H.-D., DIPADOVA, F., SCHREIER, M.H. and BRADE, H. (1993): Bakterielle Endotoxine. Beziehungen zwischen chemischer konstitution und biologischer wirkung. Immun. Infek. 21, 26 - 35.

ROBINSON, K. and FOSTER, G. (1987): Quantitative assessment of tuber contamination by pectolytic Erwinia and its possible use in the prediction and control of blackleg. Potato Research 30, $669-674$.

ROTILIO, G., CALABRESE, L., FINAZZIAGRO, A., ARGENTO-CERU, M.P., AUTUORI, F. and MONDOVI, B. (1973): Intercellular localization of superoxide dismutase and its relation to the distribution and mechanism of hydrogen peroxide producing enzymes. Biochem. Biophys. Acta 321, $98-102$.

RUDOLPH, K. (1998): Host/parasite-interactions during pathogenesis of bacterial leaf spot diseases. In Mahadevan, (ed.): Plant Pathogenic Bacteria, Proceed. $9^{\text {th }}$ Internation. Conf. Centre for Advanced Study in Botany, Univ. Madras, Chennai-India, pp. 10 - 29.

RUDOLPH, K. (1999): Host/parasite interactions in bacterial leaf spot diseases and potential mechanisms of resistance. Beiträge zur Züchtungsforschung der Bundesanstalt für Züchtungsforschung. 5, $47-54$.

SAETTLER, A.W., SCHAAD, N.W. and ROTH, D.A. (1989) : Detection of bacteria in seed and other planting material. APS Press, St. Paul, Minnesota, USA, pp. 122.

SALMOND, G.P.C. (1992): Bacterial diseases of potatoes: from classical phytobacteriology to molecular pathogenicity. Neth. J. Pl. Path. 98, Supplement 2, $115-126$.

SALMOND, G.P.C., GOLBY, P. and JONES, S. (1994): Global regulation of Erwinia carotovora virulence factor production. In: Advances in Molecular Genetics of Plant Micobe 
Interactions, ed. M.J. DANIELS, J.A. DOWNIE and A.E. OSBOURNE, Kluwer Academ. Publi., Boston, New York, USA. pp. 13 - 20.

SAMBROOK, J., FRITSCH, E.F. and MANIATIS, T. (1989): Molecular Cloning: a Laboratory Manual. Cold Spring Harbor Laboratory Press, $2^{\text {nd }}$ Edition, New York.

SANDULACHE, R. and PREHM, P. (1985): Structure of the core oligosaccharide from lipopolysaccharide of Erwinia carotovora. J. Bacteriol. 161, 1226 - 1227.

SAWARDEKER, J.S., SLONEKER, J.H. and JEANNES, A. (1965): Quantitative determination of monosaccharides as their alditol acetates by gas-liquid chromatography. Analyt. Chemie 37, $1602-1603$.

SCHOBER, B.M. and ZADOKS, J.C. (1999): Survival of soft rot bacteria during the storage of witloof Chicory roots. J. Phytopathol. 147, $461-466$.

SCHOONEJANS, E., EXPERT, D. and TOUSSAINT, A. (1987): Characterisation and virulence properties of Erwinia chrysanthemi lipopolysaccharide-defective, ФEC2-resistant mutants. J. Bacteriol. 169, $4011-4017$.

SCHRÖDER, I. (2000): Zur Rolle der Membranvesikel von Pseudomonas syringae als Resistenzinduktoren und Transportvehikel für Virulenzfaktoren bei Nicotiana tabacum und Lycopersicon esculentum. Dissertation, Universität Göttingen, 177 pp.

SCHRÖDER, I., KUHLMANN, M., DRÖGE-LASER, W., HOPPERT, M. and RUDOLPH, K. (2001): Membrane vesicle mediated transport of virulence-factors and of elicitors for defenseresponse. In: DE BOER, S.H., edit, Proceed. $10^{\text {th }}$ Internat. Conf. Plant Pathogenic Bacteria, Charlottetown, P.T., Canada, July 23 - 27, 2000.

SENCHENKOVA, S.N., KNIREL, Y.A., SHASHKOV, A.S., HUANG, X., FURUYA, N., MÜLlER, P., AHMED, M., LAUX, P. and RUDOLPH, K. (2001): Structures of the Opolysaccharide chains of the lipopolysaccharides of some phytopathogenic bacteria. Abstr., Eurocarb X1, Lissabon, Portugal. 
SENCHENKOVA, S.N., SHASHKOV, A.S., PETER, L., KNIREL, Y.A. and RUDOLPH, K. (1999): The O-chain polysaccharide of the lipopolysaccharide of Xanthomonas campestris $p v$. begoniae GSPB 525 is a partially L-xylosylated L-rhamnan. Carbohydrate Research 319, 148 153.

SHEKHAWAT, G.S. and RUDOLPH, K. (1978): Protein and hydrolytic enzymes patterns of three soft rot erwinias, obtained by disc-gel electrophoresis of soluble proteins. Phytopathol. Z. 93, $348-358$.

SHUKLA, S.D., GREEN, C., TURNER, J.M. (1980): Phosphatidylethanolamine distribution and fluidity in outer and inner membrane of the Gram-negative bacterium Erwinia carotovora. Biochem. J. 181, 131 - 135.

SIDBERRY, H., KAUFMANN, B., WRIGHT, D.C. and SADOFF, J. (1985): Immunoenzymatic analysis by monoclonal antibodies of bacterial lipopolysaccharides after transfer to nitrocellulose. J. Immunol. Methods 76, 299 - 305.

SKERMAN, V.B.D., MCGOWAN, V. and SNEATH, P.H.A. (1980): Approved lists of bacterial names. Int. J. Sys. Bacteriol. 30, 225 - 420.

SMID, E.J., JANSEN, H.J. and GORRIS, L.G.M. (1995): Detection of Erwinia carotovora subsp. atroseptica and Erwinia chrysanthemi in potato tubers using polymerase chain reaction. Plant Pathol. 44, 1058 - 1069.

STEFANI, E., DÖPKE, F. and RUDOLPH, K. (1990): Suscettibilita varietale alla $<<$ Gamba nera $>>$ della patata. Informatore Fitopatologico 10, 49 - 54.

STEINKAMP, H. (1998): Reduzierung von Mikroorganismen durch den Zusatz von Degaclean ${ }^{\circledR} 97$ zum Waschwasser beim Reinigen von Kartoffeln. Bericht Nr. 904, Projekt 98/o44, Deutsches Institut für Lebensmitteltechnik e. V., Professor-von Klitzing-Str. 7, Quakenbrück, pp. $1-7$.

STEINMANN, D. (1996): Beteiligung von Exo- und Lipopolysacchariden an der Interaktion 
von Xanthomonas campestris pv. campestris mit der Wirtspflanze Brassica oleracea und der Nicht-Wirtspflanze Capsicum annuum. Dissertation, Universität Bielefeld.

STEWART, D.J. (1962): A selective diagnostic medium for the isolation of pectinolytic organisms in the Enterobacteriaceae. Nature (London) 195, 1032.

SWINGS, J., DE VOS, P., VAN DEN MOOTER, M. and DE LEY, J. (1983): Transfer of Pseudomonas maltophilia Hugh 1981 to the genus Xanthomonas as Xanthomonas maltophilia (Hugh 1981) comb. nov. International J. System. Bacteriol. 33, 409 - 413.

THORNE, S.N. (1972): A pectate gel for the isolation and diagnosis of pectinolytic bacteria. J. Appl. Bacteriol. 35, $357-358$.

TOTH, I.K., HYMAN, L.J., GREVESSE, C. and PEROMBELON, M.C.M. (1996): An update on Erwinia diagnostics. Proceeding Crop Protection in Northern Britain, Scottish Crop Institute, Invergowrie, Dundee Scotland, U.K. pp. 331 - 336.

TOTH, I.K., THORPE, C.J., BENTLEY, S.D., MULHOLLAND, V., HYMAN, L.J., PEROMBELON, M.C.M. and SALMOND, G.P.C. (1999): Mutation in a gene required for lipopolysaccharide and enterobacterial common antigen biosynthesis affects virulence in the plant pathogen Erwinia carotovora subsp. atroseptica. Mol. Plant Microbe Interact. 6, 499 507.

VAN DEN BOOM, T. (1967): Untersuchungen über die Voraussetzungen für das Auftreten der Schwarzbeingkeit der Kartoffel. Phytopathol. Z. 58, 239 - 276.

VAN DER WOLF, J.M., HYMAN, L.J., JONES, D.A.C., GREVESSE, C., VAN BECKHOVEN, J.R.C.M., VAN VUURDE, J.W.L. and PEROMBELON, M.C.M. (1996): Immunomagnetic separation of Erwinia carotovora subsp. atroseptica from potat peel extracts to improve detection sensitivity on a crystal violet pectate medium or by PCR. J. Appl. Bacteriol. 80, 487 - 495 .

VAN SOEST, L.J.M. (1983): Evaluation and distribution of important properties in the German-Netherlands potato collection. Potato Research 26, 109 - 121. 
VAN VUURDE, J.W.L. (1990): Immunofluorescence. Bacterial colonies, p. 299 - 306. In: R. HAMPTON, E. BALL, and S.D. DE BOER (ed.), Serological methods for detection and identification of viral and bacterial plant pathogens. APS Press, St. Paul, Minn.

VAN VUURDE, J.W.L. and DE VRIES, Ph.M. (1994): Population dynamics of Erwinia carotovora subsp. atroseptica on the surface of intact and wounded seed potatoes during storage. J. Appl. Bacteriol. 76, 568 - 575.

VAN VUURDE, J.W.L. and ROOZEN, N.J.M. (1990): Comparison of immunofluorescence colony-staining in media, selective isolation on pectate medium, ELISA and immunofluorescence cell staining for the detection of Erwinia carotovora subsp. atroseptica and Erwinia chrysanthemi in cattle manure slurry. Neth. J. Pl. Path. 96, 75 - 89.

VENKATESH, B. and RUDOLPH, K. (2001): Rheological investigations on bacterial lipopolysaccharides and plant pectins. Phytomedizin, Mitteilung. Deutsch. Phytomedizin. Gesellsch. 31 (1), $39-40$.

VRUGGINK, H. and DE BOER, S.H. (1978): detection of Erwinia carotovora var. atroseptica in potato tubers with immunofluorescence following induction of decay. Potato Research 21, $225-229$.

WINN, A. M., GALBRAITH, L., TEMPLE, G.S. and WILKINSON, S.G. (1993): Structure of the $\mathrm{O} 19$ antigen of Xanthomonas maltophilia. Carbohydrate Res. 247, 249 - 254.

WOODARD, L.M., BIELKIE, A.R., EISSES, J.F. and KETCHUM, P.A. (1990): Occurrence of nitrate reductase and molybdopterin in Xanthomonas maltophilia. Appl. Environ. Microbiol. 56, $3766-3771$.

YAKOVLEVA, L.M., GVOZDYAK, R.I., ZDOROVENKO, G.M., GUBANOVA, N.Y. and SOLYANIK, L.P. (1994): Structure and functions of lipopolysaccharides from Pseudomonas syringae (serogroup II). In: Plant Pathogenic Bacteria, M. LEMATTRE, S. FREIGOUN, K. RUDOLPH and J. G. SWINGS (eds.), INRA, ORSTOM, Paris, pp. $669-674$. 
ZÄHRINGER, U., LINDNER, B. and RIETSCHEL, E.T. (1998): Chemical structure of lipid A. Recent methodical advances towards the complete structural analysis of a biologically active molecule. In: H. BRADE, D.C. MORRISON, S.M. OPAL, and S.N. VOGEL (eds.) : Endotoxin in Health and Disease, Marcel Dekker, New York, 1998.

ZDOROVENKO, G.M., GUBANOVA, N. YA., SOLYANIK, L.P., KNIREl, Y.A., YAKOVLEVA, L.M. and ZAKAROVA, I. YA. (1992): Composition and structure of lipopolysaccharides from the strains of different pathovars of Pseudomonas syringae. pp. 400 403. In: Pseudomonas syringae pathovars. 1992. Proccedings of the $4^{\text {th }}$ International Working Group on Pseudomonas syringae. Florence. Italy. 


\section{ACKNOLWEDGEMENTS}

First, I am most grateful to my supervisor Dr. Klaus Rudolph for his guidance, valuable support and confidence during this work as well as I thank him for excellent suggestions, correction, criticism during write-up, and fruitful discussion, especially for his great support during my stay in Germany.

I would like to express my sincere gratitude to Prof. Dr. H.-H. Hoppe for accepting to be my referee.

I sincerely thank Prof. Dr. U. Eberhardt for accepting to be my co-referee and his encouragement and friendly behaviour, and also Prof. Dr. Heiko Becker for his kindness in accepting me as my examiner.

I am indebted to Dr. A. Mavridis for his ever readiness to help me in the work in a friendly and amusing manner and for important suggestions and helps during laboratory and field experiments.

My special thanks go to Dr. F. Niepold, Braunschweig, Germany for allowing me to carry out PCR experiments in his laboratory. I am indebted to Dr. F. Nabizadeh-Ardekani for his advice and valuable tips in PCR techniques.

I would like to thank Dr. Y. Knirel and co-workers, Moscow, Russia, for analysing the structure of the lipopolysaccharide O-chains.

My thanks go to Dipl. Ing. agr. J. Bruer, Stöver Co., Aldrup, Germany, for providing us with the results of field experiments on the effect of Degaclean.

Due respect goes to Dr. J. Janse, Wageningen, Holland and Dr. I. Toth, Dundee, Scottland for providing me the pectins to analyse the potato samples for latent contamination.

I thank Dr. Fukuoka, Takamatsu, Japan for providing the bacterial strain (Erwinia carotovora ssp. atroseptica).

Further, I thank Dr. D. Schulz and F. Sundmacher for their friendly help to solve certain computer problems. I also thank the other staff members of the Institute and all colleagues in the "Abteilung Phytobakteriologie".

My thanks also go to the Degussa company for the co-operations and providing us with Degaclean.

I would like to thank all staff members in the Department of Plant Pathology at El-Minia University for the encouragements.

I deeply express thanks to my late father Ewis Esmael and my mother for all their efforts that they had made for my carrier.

I express my deep thanks and affection to my wife Afaf F. A. and the children (Islam, Dina and Basma) for their great support in many ways during the stay in Germany.

Finally, I thank the Government of Egypt for my financial support. 


\section{CURRICULUM VITAE}

Name

Date of birth

Place of birth

Parents

Family status

1968-1974

1974-1977

1977-1980

1980-1984

1984-1986

1986-1994

1994-1996

1996-1997

1997-2001
Mamdoh Ewis Esmael Ahmed

$15 / 08 / 1962$

Beni-Mazar, El-Minia, Egypt

Father late Ewis Esmael Ahmed and Mother Mahasen Hafez

Married since 4/ 2/ 1988

Abna El-saora Primary school, Beni-Mazar, El-Minia.

Preparatory school, Beni-Mazar, El-Minia.

Secondary school Beni-Mazar, El-Minia.

Student at the Faculty of Agriculture, El-Minia University for B. Sc.

Community service in the Army.

Master Degree (M. Sc.) in Plant Pathology at the Faculty

of Agriculture, El-Minia University and Demonstrator in

Plant Pathology.

Assisstant Lecturer in Plant Pathology at the Faculty of Agriculture.

Goethe-Institut, Bonn, Germany.

Ph. D. studies at the Department of Plant Pathology and Plant Protection of the Faculty of Agricultural Sciences, Georg-August University, Grisebachstr. 6, 37077 Göttingen, Germany. 


\section{SUMMARY}

For a sensitive detection of latent contamination of potato tubers by soft rot causing erwinias [Erwinia carotovora subsp. atroseptica (Eca), and Erwinia carotovora subsp. carotovora (Ecc)], the semi-selective double layer medium of Bdliya (1995) was improved resulting in a detection limit as low as 2 X 10 1 cfu/ml. Field experiments proved that seed potato lots assessed as moderately or strongly contaminated by Ecc gave lower yields and less quality than potato lots assessed as weakly contaminated. By applying PCR and the primer setsY1/ Y2 or Eca1f /Eca2r the detection limit of Eca in potato peel extract was $\mathbf{1 0}^{\mathbf{2}} \mathrm{cfu} / \mathrm{ml}$. Field experiments with seed potatoes treated with Degaclean revealed a reduced germination rate of the treated potatoes and increased percentage of tubers with soft rot, so that yields after treatment of seed potatoes were reduced. The repeating unit of the LPS-O-chain of $E c c 436$ was elucidated as a tetrasaccharide of the following structure:

$\rightarrow 3)-\beta-L-R h a p-(1 \rightarrow 4)-\alpha-L-R h a p-(1 \rightarrow 3)-\alpha-D-F u c p-(1 \rightarrow$

$$
\begin{array}{r}
3 \\
\uparrow \\
1 \\
\alpha-D-G l c p
\end{array}
$$

The repeating unit of the O-chain of Eca 2967 was identified as a pentasaccharide containing three galactose molecules, one rhamnose and a new higher branched sugar (similar to caryophyllose). The $E c a$-strain 2962 with a rough LPS was very weakly virulent.

\section{ZUSAMMENFASSUNG}

Für einen empfindlichen Nachweis der latenten Kontamination von Kartoffelknollen mit Weichfäule erregenden Erwinien [Erwinia carotovora subsp. atroseptica (Eca) und Erwinia carotovora subsp. carotovora (Ecc)] wurde das semi-selektive Zweischichten-Medium von Bdliya (1995) so verbessert, dass die Nachweisgrenze nur 2 X $10^{1}$ cfu/ml betrug. Feldversuche ergaben, dass Saatkartoffel-Partien, die als mittel oder stark mit Ecc kontaminiert eingestuft worden waren, nach der Ernte niedrigere Erträge von schlechterer Qualität ergaben als schwach kontaminierte Partien. Bei Anwendung der PCR mit den Primer-Paaren Y1/Y2 oder Eca1f /Eca2r betrug die Nachweisgrenze von Eca in Kartoffelschalen-Extrakten $\mathbf{1 0}^{2} \mathbf{~ c f u / m l}$. Feldversuche mit Saatkartoffeln, die mit Degaclean behandelt worden waren, ergaben eine verminderte Keimungsrate der behandelten Kartoffeln und einen erhöhten Anteil von Kartoffeln mit Weichfäule, so dass die Ernteerträge deutlich reduziert waren. Die Wiederholungseinheit der LPS-O-Kette von $\boldsymbol{E c c} 436$ wurde als ein Tetrasaccharid mit folgender Strukturformel aufgeklärt:

$\rightarrow 3)-\beta-L-R h a p-(1 \rightarrow 4)-\alpha-L-R h a p-(1 \rightarrow 3)-\alpha-D-F u c p-(1 \rightarrow$

$$
\begin{array}{r}
3 \\
\uparrow \\
1 \\
\alpha-D-G l c p
\end{array}
$$

Die Wiederholungseinheit der LPS-O-Kette von Eca 2967 wurde als ein Pentasaccharid identifiziert, das drei Galaktose-Moleküle, ein Rhamnose-Molekül und einen neuen stärker verzweigten Zucker (ähnlich der Caryophyllose) enthielt. Der Eca-Stamm 2962 mit rauher LPS war nur sehr schwach virulent. 